\title{
A Reach Control Approach to Bumpless Transfer of Robotic MANIPULATORS
}

by

Matthew Martino

A thesis submitted in conformity with the requirements for the degree of Masters of Applied Science

Graduate Department of Electrical and Computer Engineering University of Toronto

(C) Copyright 2014 by Matthew Martino 


\begin{abstract}
A Reach Control Approach to Bumpless Transfer of Robotic Manipulators

Matthew Martino

Masters of Applied Science

Graduate Department of Electrical and Computer Engineering

University of Toronto

2014
\end{abstract}

This thesis focuses on the modelling, control design, and simulations for executing a robotic manipulation task to approach and maintain contact with a solid environment. The task involves a transition between position and force control, which may result in bouncing. This thesis shows that the bumpless transfer problem (achieving no bouncing) can be formulated and solved using the reach control problem (RCP), a methodology that emphasizes the control of transient behaviour. To that end, analysis of a given force control scheme leads to the formulation of logic control specifications (a tool used in RCP designs), and a polytopic state space $\mathcal{P}$ that restricts the manipulator states to trajectories that achieve bumpless transfer. Then, a hybrid controller is developed which consists of so-called reach controllers, a given force controller, and a switching logic. Finally, the proposed methodology is simulated for the Special Purpose Dextrous Manipulator, which executes the task without bouncing. 


\section{Acknowledgements}

I still remember discussing research opportunities with Dr. Mireille Broucke in 2011 when she introduced her work in the emerging Reach Control Problem; I was hooked from the start. Under her tutelage I learned the differences between having graduated from an engineering program and being an engineer. And through my supervision she demonstrated immense patience and dedication. This was a challenging experience for me that has helped me grow in many ways, and I thank Dr. Broucke for her integral role in that process. I have great respect for the work she has done in regards to the Reach Control Problem, and wish her continuing success in its development.

This research also presented an interesting opportunity to work in the ever-advancing field of robotics. Exploring and working with the SPDM simulator was both exciting and enlightening, and I learned a great deal. So, I thank Raja Mukherji, Andrew Ogilvie, and Andrew Allen of MDA Space Missions for first introducing the heart of the problem being investigated, and for providing the SPDM simulator.

I would also like to acknowledge my fellow graduate candidates Zachary Kroeze, Ashton Roza, and Alireza Mohammadi, who were always happy and willing to lend an open ear. Moreover, I want to thank Graeme Ashford, a former MASc. candidate with Dr. Broucke, who provided some Matlab resources regarding the Reach Control Problem developed during his candidacy.

Many thanks are also in order to George Owen, the lab manager of the Motorola Controls Systems Computer Lab in the Bahen Centre for Information Technology.

My parents, Barbara and Domenic, have my unfailing gratitude for all of their love, support, and dedication throughout this experience and my entire life.

Stephanie, this was a new and challenging journey in a great number of ways. I am grateful to know that I will be able to count on you through the tough times, always. 


\section{Contents}

1 Introduction $\quad 1$

1.1 Design Intuition and Contributions $\ldots \ldots \ldots \ldots \ldots$

2 Mathematical Background $\quad 12$

2.1 Notation . . . . . . . . . . . . . . . . . . . . . . 12

2.2 Simplices and Polytopes . . . . . . . . . . . . . . . . . . . 12

2.3 Placing Triangulation $\ldots \ldots \ldots \ldots \ldots \ldots$

3 Reach Control Problem $\quad 18$

3.1 Reach Control Problem . . . . . . . . . . . . . . . . . 18

3.2 Affine Systems . . . . . . . . . . . . . . . . . . . . 23

3.3 Affine Feedback . . . . . . . . . . . . . . . . . . . 25

3.4 Triangulation and Affine Feedback . . . . . . . . . . . . . . 28

3.5 Reach Control on Polytopes . . . . . . . . . . . . . . . . . . 32

4 Three-Link Manipulator $\quad 37$

4.1 Manipulator Modelling . . . . . . . . . . . . . . . . . . 37

4.1 .1 Three-Link Manipulator Model . . . . . . . . . . . . . . . . . . . . . 39

4.2 System Modelling with Three-Link Manipulator . . . . . . . . . . . . . . . 48 
5.1 Logic Control Specifications . . . . . . . . . . . . . . . . . . . 59

5.2 Main Results . . . . . . . . . . . . . . . . . . . 65

6 Control Design for Three-Link Manipulator $\quad 81$

6.1 Reach Control Design . . . . . . . . . . . . . . . . . 81

6.2 Simulations . . . . . . . . . . . . . . . . . . . 90

7 Special Purpose Dextrous Manipulator 103

7.1 SPDM Modelling . . . . . . . . . . . . . . . . . . 103

7.1 .1 Nominal Model . . . . . . . . . . . . . . . . . . . . . . . 104

7.1 .2 Augmented Model . . . . . . . . . . . . . . . . . . . 113

7.2 System Modelling with SPDM . . . . . . . . . . . . . . 119

8 Control Design for the SPDM 132

8.1 Reach Control Design . . . . . . . . . . . . . . . . . . . . 132

8.2 Simulations . . . . . . . . . . . . . . . . . 136

9 Conclusions 152

$\begin{array}{ll}\text { Bibliography } & 157\end{array}$ 


\section{List of Tables}

5.1 System Parameters and Definitions. . . . . . . . . . . . . . . 60

6.1 Parameters of Problem 5.1 and Algorithm 5.1. . . . . . . . . . . . 84

7.1 Manipulator Joints . . . . . . . . . . . . . . . . . . . 107

7.2 Manipulator Structure . . . . . . . . . . . . . . . . . . 120

8.1 Parameters of Problem 5.1 and Algorithm 5.1 for the SPDM. . . . . . . . . . 135 


\section{List of Figures}

1.1 An example scenario of the switching problem. . . . . . . . . . 7

2.1 A 2 D simplex $\mathcal{S}$ with its vertices $v_{i}$, facets $\mathcal{F}_{i}$, and normal vectors $h_{i} \ldots \ldots \ldots$

2.2 A $2 \mathrm{D}$ polytope $\mathcal{P}$ with its vertices $v_{i}$, facets $\mathcal{F}_{i}$ and normal vectors $h_{i} \ldots \ldots$

2.3 A sample triangulation of $\mathcal{P}$ with respect to the set $\mathcal{O}[2] \ldots \ldots \ldots$

3.1 Notation for the reach control problem. . . . . . . . . . . . . . . . 19

3.2 The invariance conditions state that $y_{i}:=A v_{i}+B u_{i}+a \in \mathcal{C}\left(v_{i}\right)$ for $i=0, \ldots, n$. 21

3.3 Geometric constructs for the reach control problem. . . . . . . . . . . . . . 22

3.4 Continuous Assignment Along $\mathcal{G}$ of Vectors in $\mathcal{B}$. . . . . . . . . . . . . 31

3.5 Closed-loop vector field on $\mathcal{S}$ for Example $3.4 \ldots \ldots \ldots$. . . . . . . . . . 32

4.1 A vector $r$ in frame $F_{0}$ with coordinate axes $x_{0}, y_{0}, z_{0}$ (pointing out of the page). Frame $F_{1}$ rotated by the angle $\theta$ with respect to Frame $F_{0} \ldots \ldots \ldots$

4.2 Notation for a Three-Link Planar Manipulator . . . . . . . . . . . . . . . . 40

4.3 Control in Joint Space: (a) Joint Response, (b) Position Response . . . . . . . . 47

4.4 Control in Task Space: (a) Joint Response, (b) Position Response . . . . . . . . 47

4.5 Three-link Manipulator with second mass $m_{2} \ldots \ldots \ldots$. . . . . . . . 48

4.6 End effector Frame and the Spring-Force connecting the Second Mass. . . . . . . 51

4.7 Two-mass model consisting of the feedback-linearised manipulator, $m_{1}$, and second mass $m_{2} \ldots \ldots \ldots \ldots \ldots \ldots \ldots \ldots \ldots \ldots$

5.1 The state space $\mathcal{P}$ of $\left(\xi_{1}, \xi_{2}\right)$ according to the Logic Control Specifications. . . . . 63

5.2 The subdivision of $\mathcal{P}$ and intended directions of travel across exit facets $\mathcal{F}_{i}$. . . 66 
5.3 Salient variables for region $\mathcal{P}^{3}$, and the polytope $\mathcal{P}^{\prime}$ in the proof of Lemma 5.1. . 70

5.4 The level set $V(\xi)=c^{*}$ and invariant set $\Omega^{*}$ of Lemma $5.2 \ldots \ldots \ldots$

6.1 The triangulated state space with desired closed-loop velocity vectors at the

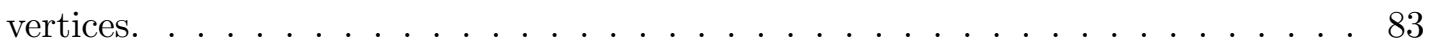

6.2 Simplex $\mathcal{S}^{\prime}$ used in the design of the reach controller for $\mathcal{P}^{2} \ldots \ldots \ldots$

6.3 Closed-loop vector field of $\mathcal{P}$ under $u_{\text {rcp }} \ldots \ldots \ldots \ldots$. . . . . . . . . 89

6.4 Closed-loop vector field of $\mathcal{P}^{3}$ under $u_{r c p}^{10} \ldots \ldots \ldots \ldots$. . . . . . . . 89

6.5 (a) Trajectory of $\xi$ of the first mass in $\mathcal{P}^{1}$. (b) Trajectory of $\xi$ in $\mathcal{P}^{1}$ and $\mathcal{P}^{2}$. . 90

6.6 Trajectory of $\xi$ of the first mass in $\mathcal{P}^{3} \ldots \ldots \ldots \ldots$. . . . . . . . . 90

6.7 (a) Trajectory of $\left(x_{2}, \dot{x}_{2}\right)$ of the second mass in $\mathcal{P}^{1}$ and $\mathcal{P}^{2}$. (b) Trajectory of $\left(x_{2}, \dot{x}_{2}\right)$ of the second mass in $\mathcal{P}^{3} \ldots \ldots \ldots \ldots$. . . . . . . . . . . 92

6.8 Highest environment stiffness $k_{e}=5 \cdot 10^{7} \mathrm{~N} / \mathrm{m}$ : (a) Constrained trajectory of $\xi$. (b) Constrained trajectory of $\left(x_{2}, \dot{x}_{2}\right)$. (c) Sensed force $f_{s}$ and environmental

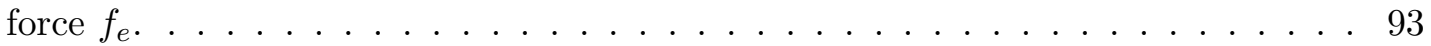

6.9 Mid-range environment stiffness $k_{e}=10^{7} \mathrm{~N} / \mathrm{m}$ : (a) Constrained trajectory of $\xi$.

(b) Constrained trajectory of $\left(x_{2}, \dot{x}_{2}\right)$. (c) System forces $f_{s}$ and $f_{e} \ldots \ldots 93$

6.10 Lowest environment stiffness $k_{e}=10^{6} \mathrm{~N} / \mathrm{m}$ : (a) Constrained trajectory of $\xi$. (b) Constrained trajectory of $\left(x_{2}, \dot{x}_{2}\right)$. (c) System forces $f_{s}$ and $f_{e} \ldots \ldots . \ldots 9$

6.11 (a) Index of active sub-controller of $u\left(\xi, f_{s}\right)$. (b) Manipulator joint torques $\tau$. . . 95

6.12 (a) Manipulator joint angles $\theta$ and orientation angle $\phi$. (b) Cartesian coordinates of the second mass $x_{2}$ and $y_{2} \ldots \ldots \ldots \ldots \ldots \ldots \ldots$

6.13 Sensed force $f_{s}$ for varying reach control design parameter $\alpha . \ldots \ldots$

6.14 (a) Trajectories $\xi$ of the first mass in $\mathcal{P}^{2}$ and $\mathcal{P}^{3}$ for different mass ratios. (b)

The sensed force $f_{s}$ for different mass ratios. . . . . . . . . . . . 97

6.15 Sensed force $f_{s}$ for varying environment position $\rho_{e} \ldots \ldots \ldots$

6.16 Below nominal environment stiffness $k_{e}=10^{5} \mathrm{~N} / \mathrm{m}$ : (a) Constrained trajectory $\xi$ of the first mass. (b) System forces $f_{s}$ and $f_{e} \ldots \ldots \ldots$. . . . . . 99

6.17 Above nominal environment stiffness $k_{e}=10^{8} \mathrm{~N} / \mathrm{m}$ : (a) Constrained trajectory $\xi$ of the first mass. (b) System forces $f_{s}$ and $f_{e} \ldots \ldots \ldots$. . . . . . 100 
6.18 Above nominal environment stiffness $k_{e}=10^{8} \mathrm{~N} / \mathrm{m}$ : (a) Constrained trajectory $\left(x_{2}, \dot{x}_{2}\right)$ of the second mass. (b) System forces $f_{s}$ and $f_{e}$ close to the switching boundary. . . . . . . . . . . . . . . . . . . . 101

6.19 Below nominal sensor stiffness $k_{s}=5 \cdot 10^{5} \mathrm{~N} / \mathrm{m}$ : (a) System forces $f_{s}$ and $f_{e}$. (b) System forces $f_{s}$ and $f_{e}$ close to the switching boundary. . . . . . . . 101

7.1 Notation and directions for the FMS frame. . . . . . . . . . . . . . . . 104

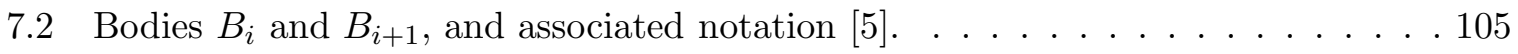

7.3 SPDM Top View $[38] . \ldots \ldots \ldots \ldots \ldots$

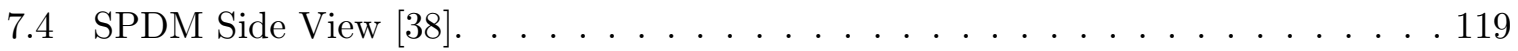

7.5 Simplified Three-link Manipulator of the SPDM. . . . . . . . . . . . . . 121

8.1 Closed-loop vector field of $\mathcal{P}$ under $u_{r c p} \ldots \ldots \ldots$. . . . . . . . . . . . . . . .

8.2 (a),(b) Trajectory of $\xi$ of the first mass in $\mathcal{P}^{1}$ and $\mathcal{P}^{2} \ldots \ldots \ldots \ldots$

8.3 Trajectory of $\xi$ of the first mass in $\mathcal{P}^{3} \ldots \ldots \ldots \ldots$. . . . . . . . . . . . . . .

8.4 (a) Trajectory of $\left(x_{2}, \dot{x}_{2}\right)$ of the second mass in $\mathcal{P}^{1}$ and $\mathcal{P}^{2}$. (b) Trajectory of $\left(x_{2}, \dot{x}_{2}\right)$ of the second mass in $\mathcal{P}^{3} \ldots \ldots \ldots \ldots \ldots$

8.5 Highest environment stiffness $k_{e}=5 \cdot 10^{8} \mathrm{~N} / \mathrm{m}$ : (a) Constrained trajectory of $\xi$. (b) Constrained trajectory of $\left(x_{2}, \dot{x}_{2}\right)$. (c) Sensed force $f_{s}$ and environmental

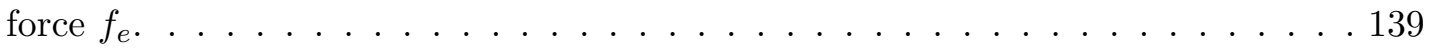

8.6 Mid-range environment stiffness $k_{e}=2.1 \cdot 10^{8} \mathrm{~N} / \mathrm{m}$ : (a) Constrained trajectory of $\xi$. (b) Constrained trajectory of $\left(x_{2}, \dot{x}_{2}\right)$. (c) System forces $f_{s}$ and $f_{e} \ldots \ldots 139$

8.7 Lowest environment stiffness $k_{e}=10^{7} \mathrm{~N} / \mathrm{m}$ : (a) Constrained trajectory of $\xi$. (b) Constrained trajectory of $\left(x_{2}, \dot{x}_{2}\right)$. (c) System forces $f_{s}$ and $f_{e} \ldots \ldots 139$

8.8 FMS sensed force $F_{y}$ and moment $M_{z}$ during contact. . . . . . . . . . 141

8.9 (a) Index of active sub-controller of $u\left(\xi, f_{s}\right)$. (b) Manipulator joint torques $\tau$. . . 142

8.10 (a) Manipulator joint angles $\theta$ and orientation angle $\phi$. (b) Cartesian coordinates of the first mass $x_{1}, y_{1}$ and second mass $x_{2}, y_{2} \ldots \ldots \ldots \ldots \ldots$

8.11 Return Approach: (a) State of control law $u\left(\xi, f_{s}\right)$. (b) System forces $f_{s}$ and $f_{e} .143$

8.12 Return Approach: Closed-loop vector trajectory of $\xi \ldots \ldots$. . . . . . . . . 144

8.13 Sensed force $f_{s}$ for varying reach control design parameter $\alpha . \ldots \ldots . \ldots 144$ 
8.14 (a) Trajectories $\xi$ of the first mass in $\mathcal{P}^{1}$ and $\mathcal{P}^{2}$ for different mass ratios. (b) The sensed force $f_{s}$ for different mass ratios. . . . . . . . . . . . 145

8.15 Below nominal sensor stiffness $k_{s}=2.1 \cdot 10^{6} \mathrm{~N} / \mathrm{m}$ : (a) System forces $f_{s}$ and $f_{e}$.

(b) System forces $f_{s}$ and $f_{e}$ close to the switching boundary. . . . . . . 146

8.16 (a) Constrained motion of $\xi$ for greater structural damping, $z=0.05$. (b) System forces $f_{s}$ and $f_{e}$ for greater structural damping, $z=0.05 \ldots \ldots \ldots$. . . . 147

8.17 Sensed force $f_{s}$ for varying environment position $\rho_{e} . \ldots \ldots \ldots$

8.18 Below nominal environment stiffness $k_{e}=7.5 \cdot 10^{6} \mathrm{~N} / \mathrm{m}$ : (a) Constrained trajectory $\xi$ of the first mass. (b) System forces $f_{s}$ and $f_{e} \ldots \ldots \ldots$. . . . . 149

8.19 Above nominal environment stiffness $k_{e}=10^{9} \mathrm{~N} / \mathrm{m}$ : (a) Constrained trajectory $\xi$ of the second mass. (b) System forces $f_{s}$ and $f_{e} \ldots \ldots \ldots$. . . . . . . . .

8.20 Above nominal environment stiffness $k_{e}=10^{9} \mathrm{~N} / \mathrm{m}$ : (a) Constrained trajectory $\left(x_{2}, \dot{x}_{2}\right)$ of the first mass. (b) System forces $f_{s}$ and $f_{e}$ close to the switching boundary. . . . . . . . . . . . . . . . . . . . 150 


\section{Chapter 1}

\section{Introduction}

This thesis focuses on the modelling, control design, and simulations for achieving a specific maneuver of a robotic manipulator. During the maneuver the end effector of the manipulator approaches and reaches a solid environment in its workspace, and maintains contact with the environment at a specified contact force. Such a maneuver may be called an interaction task. The system under consideration may be classified as a hybrid system since it requires a transition between a position control scheme in free motion and a force control scheme during constrained motion. Research into robotic manipulation and interaction tasks is plentiful, with emphasis on the transition between the free motion and constrained motion phases. In particular, control schemes may be developed to optimize one or more of the factors crucial to robotic interaction tasks including peak contact forces, stability, and bouncing. In addition to the execution of the full interaction task, this thesis focuses on bouncing, which is defined as an undesired switch between the free motion and constrained motion control schemes. Specifically, the development to follow assumes that the constrained motion phase is already fully specified; that is, a stable force feedback law is assumed for simple force regulation. The objective then is to design a set of position controllers to act during the transition and free motion phases, and to define a switching logic that yields a 'bounceless' transition from free to constrained motion, a behaviour that may be referred to as a bumpless transfer.

One of the central themes of this thesis is to show that the bumpless transfer problem can be formulated and solved within the context and principles of the reach control problem (RCP). RCP provides a methodology to address control problems that go beyond the traditional 
stabilization and regulation requirements. It does so by placing emphasis on the control of the transient behaviour. The desired transient behaviour is characterized primarily through two elements: linear inequalities that describe state constraints and a qualitative description of temporal events. Together these two elements are known as "logic control specifications". Specifically, the linear inequalities are used to restrict the operational state space of the system variables to a polytope $\mathcal{P}$ bounded in $\mathbb{R}^{n}$. Such constraints naturally lend themselves to the realization of strict safety requirements in order to operate within tolerable technology limits or to evolve through a staged process in a more controlled manner. One then further divides $\mathcal{P}$ into a set of simplices by means of triangulation. Roughly speaking, the role of RCP is to find controllers to guide trajectories of the closed-loop system through a sequence of simplices, thereby obeying the temporal description, while always remaining inside $\mathcal{P}$.

The reach control problem was pioneered by Habets and van Schuppen in 2001 in [28]. They provided necessary and sufficient conditions for a more restricted version of RCP by a given affine feedback on a simplex in [29]. They also provided a synthesis procedure to obtain the affine feedback using only information about the control input at the vertices of the simplex [29]. The modern formulation of RCP, relaxing the requirement of [29] that trajectories exit at the first time they reach the exit facet, appeared simultaneously in [31] and [30]. In these works, two necessary and sufficient conditions, the so-called invariance conditions and flow condition appeared. The invariance conditions place restrictions on the velocity vectors at the vertices of the simplex so that closed-loop trajectories may not exit restricted facets (facets which are not an exit facet). The flow condition guarantees that closed-loop trajectories do exit the simplex.

A more geometric approach was pursued in [33]. First, it was observed that the flow condition was equivalent to the condition that the closed-loop vector field has no equilibria in $\mathcal{S}$. Using the fact that for affine systems equilibria can only appear on an affine set called $\mathcal{O}$, the polytopic state space is partitioned with respect to $\mathcal{O}$; in particular, $\mathcal{O}$ may not intersect the interior of any simplices of the triangulation of the polytope. Under this triangulation assumption it is shown in [33] that continuous state feedback and affine feedback are equivalent with respect to solvability of RCP. New necessary and sufficient conditions for solvability of $\mathrm{RCP}$ in terms of the problem data are given. This leads to a synthesis method for affine feedbacks under the proposed triangulation assumption. Extensions of the geometric approach 
from simplices to polytopes were presented in [2].

Thus far the techniques of RCP have generated success in a number of applications, i.e. control problems that are described by a more demanding set of complex specifications. In particular, RCP was applied in the control design of an automated anaesthesia delivery system for both second- and third-order plants [4]. In [3] a material transfer system consisting of a cart between conveyors was the subject of time-varying feedbacks on simplices. In [1], a Two-Tank Problem involving temperature control for a chemical process was solved using a simplex-based approach. The design was improved upon in [2] using the technique of the Monotonic Reach Control Problem (MRCP), which removed the dependence of a solution on the triangulation, and removed the use of discontinuous feedbacks.

The advantage of RCP, as demonstrated in the above works, is its ability to achieve the desired temporal behaviour while restricting the system states to the region $\mathcal{P}$ defined by the problem specifications, so long as the states are initialized within $\mathcal{P}$. That is to say that RCPbased designs exhibit a robustness to variations in initial conditions within their operating range. As a result, a system can be guaranteed to perform within stated safety or behavioural constraints. This ability is not easily replicated by standard stabilization methodologies. For example, the reader is referred to Chapter 4 of [1] in which a case study is given that compares RCP to common stabilization methods for the control of the aforementioned material transfer system, and of a chemical process application. It was shown that the synthesized stabilization controllers could only complete the primary objective and adhere to the safety constraints over a limited set of initial conditions in $\mathcal{P}$. A continuous redesign of the stabilization controllers or a more complex switching logic would be required to match the reliability of the RCP-based design [1].

This thesis then aims to exploit the advantages of RCP in the control of a manipulator, particularly in the free motion and transition phases of the interaction task. Specifically, a general set of logic control specifications will be defined that restrict the allowable position and velocity of the end effector in relation to its proximity to the environment. Out of these specifications a polytopic state space $\mathcal{P}$ describing the allowable operating region will be obtained. Once a switching logic has been found which achieves a bumpless transfer between force control and position control (henceforth called reach control), reach controllers are designed such that 
any end effector trajectory starting in $\mathcal{P}$ will evolve to a point where contact is made and the desired switching logic is satisfied. The desired force regulation follows.

Now we return our attention to the bouncing problem of manipulators. Interaction tasks pose a challenging control problem for achieving stable contact with an environment. As discussed in the literature, [6], [8], [12], initial contact with an environment may lead to instability and bouncing of the manipulator for reasons including, large impact forces that are not compensated, incompatible initial conditions, or the change in system dynamics. To combat these issues robotic control techniques have been developed that can be broadly classified as continuous or unified, or as discontinuous or switching. For example, Hogan's Impedance Control [15], is a continuous control scheme that produces a single controller for the free, transition, and contact phases. The controller is designed such that the manipulator exhibits a particular output impedance to match the parameters of a work environment, inherently providing stable contact. As indicated in [8] however, an accurate environment model is required and must be accounted for in the free motion design, otherwise force regulation is not feasible upon contact.

In contrast, a discontinuous switching system explicitly changes between free and constrained controllers. This control structure allows each controller to be specialized to perform a specific task, but requires extra attention regarding the transition between the controllers. With respect to the bouncing problem, a number of discontinuous control schemes are reviewed below, classified according to the strategy used to alleviate bouncing and achieve bumpless transfer. All of the schemes employ a force control algorithm called explicit force control, where the controller input is the error between measured and desired contact forces.

(i) Construct a controller for the transition phase: Under this approach, an intermediate controller is generally tailored to the system behaviour during or immediately after impact. The objective is to alter the state of the system such that the constrained controller can then take over and safely perform force regulation. In [6], once impact is detected an intermediate controller is enabled that regulates to zero the velocity and acceleration normal to the impact location. Afterwards, a force controller is enabled to regulate the contact force. In [10] a proportional force controller is used upon impact, after which integral control is adopted for regulation. Similarly, in [9] a timed velocity feedback 
is applied upon impact to dissipate energy, after which a standard feedback is applied. Lastly, in [14] six regions of motion are identified for the transition from free to constrained motion for which a discontinuous control scheme is derived.

(ii) Use non-standard feedback terms: The assumed 'standard' feedback elements include feedforward, proportional, integral and velocity feedback terms. Feedbacks outside of that scope are generally used to compensate for more complex dynamics, or to achieve additional functionality. For example in [8], a partial acceleration feedback term is specifically included in the constrained controller to make the force dynamics dominate the system behaviour; the remaining feedback elements may then be tuned to achieve a bounceless transition. Note that [14] also falls into this category for some of the discontinuous feedback elements employed.

(iii) Experimentation and Parameter Tuning: Approaches that depend on parameter tuning or timed control actions are generally more difficult to employ. In [13] analysis and experimentation of an anti-windup integral controller for the transition and constrained phases of motion was performed. Note that in [8] and [9] some parameter tuning is also required to achieve a bounceless transition.

(iv) Derive sufficient conditions for bumpless transfer: A particularly interesting approach is to first identify system trajectories that result in a bumpless transfer, and then to construct a solution that guarantees that only these behaviours occur. For example, in [7] an invariant set is constructed with respect to the constrained dynamics, such that trajectories that enter this region do not produce bounces. Admissible free motion trajectories are then constrained to those which automatically enter the invariant set. On the other hand, in [11] an expression for the maximum number of expected bounces is derived based on the proposed solution, from which a sufficient condition for a bounceless transition is easily obtained. In both cases, achieving a bounceless transition is correlated to limiting impacts to a maximum velocity.

From the reviewed works, that of Teel et al [7] stands out as the most relevant and inspirational to the development that follows. The work has two objectives: i) achieve bumpless 
transfer from position to force control; ii) compensate for measurement noise that can cause undesired control switches (hysteresis is incorporated in the solution to address this objective). A spring-damper environment model is defined by known parameters $k_{c}$ and $b_{c}$ respectively, such that the contact force is expressed in terms of the end effector states. Then, given a desired contact force, a Lyapunov function is used to design a feedforward and proportional force controller. To address bouncing, an invariant set is selected that is a sub-level set of the previous Lyapunov function, the largest that is completely contained within the environment. Switching boundaries representing lines of constant contact force are then designed; the lines are slanted with slope $-\frac{k_{c}}{b_{c}}$. To implement hysteretic control, two switching boundaries are selected, defined by forces $\gamma_{2}, \gamma_{1}$, where $0<\gamma_{1}<\gamma_{2}$. The boundary for $\gamma_{2}$ is located within the aforementioned sub-level set, while the other is generally located on the boundary of the set. A key point to the methodology is that the position controller to be designed must be strong enough to generate the contact force $\gamma_{2}$ to induce a switch to force control. To meet this requirement, a co-design of the $\gamma_{i}$ and the $\mathrm{PD}$ position controller occurs: first the proportional parameter $k_{p}$ is selected, which then informs the design of $\gamma_{2}$. Having obtained the invariant set and switching boundaries, a maximum impact velocity $x_{2}^{*}$ is identified such that trajectories reaching the environment below $x_{2}^{*}$ are guaranteed to enter the selected invariant set. An explicit formula for $x_{2}^{*}$ is not given, but it appears to depend on the minimum possible value of $\gamma_{2}$. Next the free motion controller design is completed by selecting the damping term $k_{d}$ arbitrarily. Then, as per the strategy explained in (iv), Lyapunov analysis pertaining to free motion yields a computation of the set of initial conditions that guarantees an impact velocity below $x_{2}^{*}$, denoted $K_{0}$. This set is necessarily contained in a level set, and its size depends on the environment parameters. For example, as the stiffness of the environment increases, the set of initial conditions that guarantees a safe impact velocity shrinks. While the entire design methodology is intriguing, it is desirable to overcome the dependence of $K_{0}$ on the environment, and to provide a methodology that accommodates a less restrictive environment definition. 


\subsection{Design Intuition and Contributions}

We begin this section by describing the main ideas and contributions of the thesis in informal terms in order to prepare the reader for the more technical results to follow. We consider a robotic manipulator and an environment whose position $\hat{\rho}_{e}$ is not known exactly but is contained within a known band of values $\hat{\rho}_{e} \in\left[\hat{\rho}_{\text {emin }}, \hat{\rho}_{\text {emax }}\right]$. The environment is modelled by a spring with stiffness $k_{e}$; the exact value of $k_{e}$ is unknown but it varies within a known range $k_{e} \in\left[k_{\text {emin }}, k_{\text {emax }}\right]$. It is assumed that a force controller has been given apriori whose task is to regulate the contact force to a desired reference value $f_{s}^{d}$.

Suppose that a hybrid controller has been designed which consists of two controllers: a position controller that guides the manipulator to the environment and the given force controller. Suppose the switching logic between the two controllers is simply to switch from position control to force control when the sensed force reaches a threshold $f_{s w}$. Using a model for the sensed force, the force $f_{s w}$ can be related to a position $\rho_{s w}$ of the manipulator so that the switching logic can be interpreted in terms of the position of the manipulator.

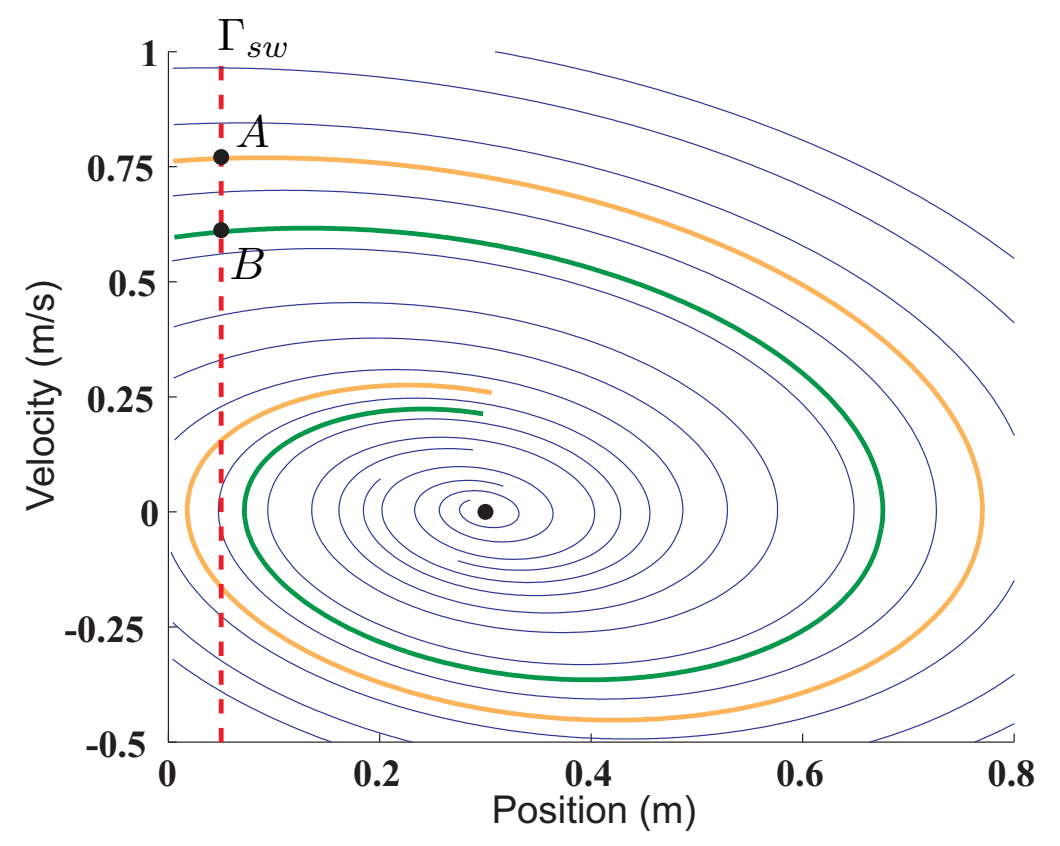

Figure 1.1: An example scenario of the switching problem.

Now consider Figure 1.1. It shows the position vs velocity of the end effector. To the right of the line $\Gamma_{s w}$ which corresponds to $\rho_{s w}=\frac{\left|f_{s w}\right|}{k_{e}}$, the phase portrait of the closed-loop vector field 
under force control is shown. Closed-loop phase curves tend to an equilibrium corresponding to the desired steady-state contact force $f_{s}^{d}$. Suppose that under position control the manipulator state reaches $\Gamma_{s w}$ at point $\mathrm{A}$, at which time the force controller is enabled. The resulting trajectory, in orange, crosses $\Gamma_{s w}$ into the region to the left of $\Gamma_{s w}$, where the position controller will be re-enabled. This behaviour corresponds to "bouncing" between position control and force control. In contrast, suppose that under position control the manipulator state reaches $\Gamma_{s w}$ at point $\mathrm{B}$. The resulting trajectory, in green, remains to the right of $\Gamma_{s w}$ and force regulation is achieved without additional switches.

Evidently the force controller has no functionality to determine whether bouncing will occur, nor to avoid it. Rather, it is the task of the position controller to ensure that only certain states along the boundary $\Gamma_{s w}$ are reached. This is the main idea of the design presented in this thesis: to design reach controllers to guarantee that the manipulator state does not arrive at points corresponding to closed-loop trajectories under force control that result in bouncing. Now we introduce, again in informal terms, the main ideas of the control design.

The first phase of the control design is to perform a Lyapunov analysis of the closed-loop force dynamics. Specifically, a quadratic Lyapunov function for the linear stable closed-loop force dynamics is found. Then a sub-level set of this Lyapunov function is identified which allows one to construct an invariant set $\Omega$ that lies entirely to the right of the switching boundary $\Gamma_{s w}$. Because this invariant set lies entirely to the right of the switching boundary $\Gamma_{s w}$, if trajectories can be driven into this set, then they cannot return to the left side of $\Gamma_{s w}$ and position control. The most important feature of the invariant set $\Omega$ is that it intersects the switching line $\Gamma_{s w}$ along a vertical segment corresponding to manipulator velocities in the range $\left[0, \mu_{s w}\right]$. The parameter $\mu_{s w}$ serves as an upper bound on the velocity of the manipulator at the time of impact with the environment to guarantee that there will be no bouncing. To reiterate, trajectories that cross the switching line $\Gamma_{s w}$ with velocity bounded by $\mu_{s w}$ enter the invariant set $\Omega$, and do not cross the switching line again in the opposite direction; that is, bumpless transfer is achieved. Notice that $\mu_{s w}$ is a parameter that is extracted through the analysis. Our methodology actually extends this analysis to one that holds over the entire set of environment parameters.

The second phase of the control design involves defining a polytopic state space $\mathcal{P}$ (which 
depends on the parameter $\left.\mu_{s w}\right)$, triangulating $\mathcal{P}$, and specifying reach controllers on each simplex of the triangulation to achieve the control objective. The desired behaviour of the reach controllers is determined from a set of logic specifications which state how trajectories must move through the polytopic state space. This state space is determined both by parameters that are set by the designer at the outset, including maximum speed and minimum distance from the environment, as well as parameters that come out of the analysis in the first phase. These include position and velocity constraints during impact and in free motion, and they are directly informed by the investigation of the bouncing problem. The method for choosing these other parameters is summarized in an algorithm, which when executed determines the admissible state space $\mathcal{P}$. In this sense $\mathcal{P}$ serves to constrain the manipulator states and trajectories to those that exhibit a bumpless transfer from reach to force control. Finally, the combination of the reach controllers, proposed switching logic, and given force controller constitute an overall hybrid control that oversees the interaction task.

To validate the proposed solution an interaction task will be simulated for the Special Purpose Dextrous Manipulator (SPDM), which is a robotic system that operates aboard the International Space Station. The SPDM is responsible for a number of spaced-based construction and maintenance tasks that require strict safety and performance measures to be met. As such, the SPDM is an ideal system with which to demonstrate the advantages of an RCPbased design. The SPDM is designed by MDA Space Missions (henceforth called MDA), which has provided Matlab and Simulink models of the SPDM to assist with the control design and simulations.

This thesis is organized as follows. Chapter 2 presents the necessary mathematical background for the objects and tools employed by the reach control problem, such as simplices and triangulations. Chapter 3 introduces and develops the reach control problem by examining the core results for its solvability in regards to the invariance and flow conditions, as well as the existence of equilibria. Solutions to RCP on both simplices and polytopes are presented. In Chapter 4 the non-linear dynamical model for a planar three-link manipulator is presented, and simplified to obtain a model amenable to control design. The derivation includes a reduction of the original manipulator model to a simpler two-mass model. It is worth noting that this two-mass model is sufficiently general to capture any manipulator model. The contributions 
of the thesis begin with the reduction of the two-mass model to a single-mass model which is amenable to control design. From this single-mass system a number of state space models are identified, corresponding to the different phases of manipulator motion. A solution to the bouncing problem is derived using these state space models. Chapter 5 formally presents the problem to be solved, as well the core methodology for its solution. In Chapter 6 the control design process is given in the context of an arbitrary three-link manipulator, using the solution of Chapter 5. The system is then simulated to validate the design. Next, the focus of the thesis shifts to the SPDM. In Chapter 7 the dynamical model of the SPDM employed by MDA is presented and then simplified along the lines of Chapter 4 to obtain a single-mass system. The control design and simulation process is then repeated for the SPDM in Chapter 8. Lastly, concluding statements and insight are detailed in Chapter 9. This thesis contains the following contributions.

- Chapter 4 presents the derivation of a simplified, linear single-mass system from a nonlinear, planar three-link manipulator, for which a solution to the bouncing problem is derived. The derivation includes a reduction of the original manipulator model to a simpler two-mass model, which is general, and then the contributions of this thesis begin through the reduction of the two-mass model to a single-mass system. From here, multiple state space models are identified, corresponding to the different phases of manipulator motion.

- Chapter 5 formulates the interaction task in the context of the reach control problem, which includes the definition of a set of logic control specifications. Additionally, the solution methodology is presented, which can be summarised into three categories: (1) A no-bouncing solution is obtained in the design of the switching logic between free and constrained motion, using Lyapunov techniques; (2) An operating region $\mathcal{P}$ is obtained that constrains system states and trajectories to those that exhibit a bumpless transfer; (3) A set of reach controllers are synthesised that, (i) guides the manipulator upon contact to the switching boundary defined in (1), and (ii) ensures that the states of the end effector conform to the logic control specifications during the free and transition phases.

- In Chapter 6, control design is performed for an arbitrary manipulator in which parameter 
selection is unconstrained. The design is then validated via simulation.

- In Chapter 7, the dynamical model of the SPDM is presented and then adapted to the form given in Chapter 4, for which the methods of Chapter 5 may be applied.

- Finally, in Chapter 8 the proposed methodology and control design are applied to the SPDM, where certain parameters are already constrained. Simulations similar to those in Chapter 6 are then discussed. 


\section{Chapter 2}

\section{Mathematical Background}

\subsection{Notation}

Let $\mathcal{K} \subset \mathbb{R}^{n}$ be a set. The complement of $\mathcal{K}$ is $\mathcal{K}^{c}:=\mathbb{R}^{n} \backslash \mathcal{K}$, the closure is $\overline{\mathcal{K}}$, and the interior is $\mathcal{K}^{\circ}$. For a vector $x \in \mathbb{R}^{n}$, the notation $x \succ 0(x \succeq 0)$ means $x_{i}>0\left(x_{i} \geq 0\right)$ for $1 \leq i \leq n$. The notation $x \prec 0(x \preceq 0)$ means $-x \succ 0(-x \succeq 0)$. For a matrix $A \in \mathbb{R}^{n \times n}$, the notation $A \succ 0(A \succeq 0)$ means $a_{i j}>0\left(a_{i j} \geq 0\right)$ for $1 \leq i, j \leq n$. Notation $\mathbf{0}$ denotes the subset of $\mathbb{R}^{n}$ containing only the zero vector. The notation $\mathscr{B}$ denotes the open unit ball, and $\overline{\mathscr{B}}$ denotes its closure. The notation $\operatorname{co}\left\{v_{1}, v_{2}, \ldots\right\}$ denotes the convex hull of a set of points $v_{i} \in \mathbb{R}^{n}$. The notation aff $\left\{v_{1}, v_{2}, \ldots\right\}$ denotes the affine hull of a set of points $v_{i} \in \mathbb{R}^{n}$. The symbol $\mathbb{U}$ denotes a control type: we consider open-loop controls, continuous state feedback, affine feedback, and multiaffine feedback. The notation $\mathbb{S}^{n}$ denotes the unit sphere $\left\{x \in \mathbb{R}^{n}:\|x\|=1\right\}$.

\subsection{Simplices and Polytopes}

The standard $n$-simplex is the subset of $\mathbb{R}^{n+1}$ given by

$$
\Delta^{n}:=\left\{\left(\alpha_{0}, \ldots, \alpha_{n}\right) \in \mathbb{R}^{n+1} \mid \sum_{i} \alpha_{i}=1, \alpha_{i} \geq 0\right\}
$$

More generally, an $n$-dimensional simplex is an $n$-dimensional polytope with $n+1$ vertices. Let

$$
V:=\left\{v_{0}, v_{1}, \ldots, v_{n}\right\}
$$


be a set of $n+1$ points in $\mathbb{R}^{n}$. We say $\left\{v_{0}, \ldots, v_{n}\right\}$ are affinely independent if they do not lie in an $(n-1)$-dimensional plane in $\mathbb{R}^{n}$. Equivalently, $\left\{v_{0}, \ldots, v_{n}\right\}$ are affinely independent if $\left\{v_{1}-v_{0}, \ldots, v_{n}-v_{0}\right\}$ are linearly independent. This provides another way to define a simplex: an $n$-dimensional simplex is the convex hull of $n+1$ affinely independent points in $\mathbb{R}^{n}$. The affine hull of $V$, denoted aff $\{V\}$, is the smallest affine space containing $V$.

Suppose that $V$ is affinely independent and define the simplex

$$
\mathcal{S}:=\operatorname{co}\left\{v_{0}, v_{1}, \ldots, v_{n}\right\}
$$

A face of $\mathcal{S}$ is any sub-simplex which makes up its boundary. An $(n-1)$-dimensional face of $\mathcal{S}$ is called a facet. We denote the facets of $\mathcal{S}$ by $\mathcal{F}_{0}, \ldots, \mathcal{F}_{n}$. Our numbering convention is such that

$$
\mathcal{F}_{i}=\operatorname{co}\left\{v \in V \backslash\left\{v_{i}\right\}\right\}, \quad i \in\{0,1, \ldots, n\} .
$$

That is, each facet is indexed by the vertex it does not contain. Let $h_{i}$ denote the unit normal vector to $\mathcal{F}_{i}$, pointing out of $\mathcal{S}$. A simplex is depicted in Figure 2.1, illustrating the notation used. An implicit description of $\mathcal{S}$ is obtained using the normal vectors $h_{i}$. Namely, there exist $\alpha_{0}, \ldots, \alpha_{n} \in \mathbb{R}$ such that

$$
\mathcal{S}=\left\{x \in \mathbb{R}^{n} \mid h_{i} \cdot x \leq \alpha_{i}, \quad \forall i \in\{0, \ldots, n\}\right\}
$$

Finally we collect some useful properties about simplices.

Lemma 2.1. Let $\mathcal{S}$ be a simplex. Then the following hold:

(i) If $x \in \operatorname{co}\left\{v_{1}, \ldots, v_{k}\right\}$, then $x \in \mathcal{F}_{j}$, for $k+1 \leq j \leq n$.

(ii) $h_{j} \cdot\left(v_{i}-v_{0}\right)=0$ for all $1 \leq i, j \leq n$ and $j \neq i$.

(iii) $h_{j} \cdot\left(v_{i}-v_{k}\right)=0$ for all $0 \leq i, k \leq n$ and $j \neq i, k$.

(iv) $h_{i} \cdot\left(v_{i}-v_{0}\right)<0$, for all $1 \leq i \leq n$.

(v) $h_{j} \cdot\left(v_{i}-x\right)>0$ for all $x \in \mathcal{S} \backslash \mathcal{F}_{j}$ and $1 \leq i, j \leq n$ and $i \neq j$.

(vi) $h_{0} \cdot\left(v_{i}-v_{0}\right)>0$ for all $1 \leq i \leq n$. 


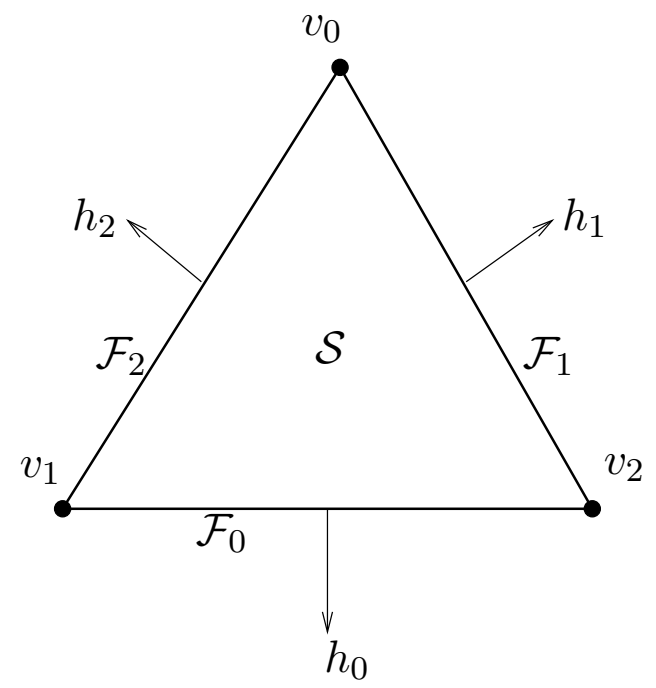

Figure 2.1: A 2D simplex $\mathcal{S}$ with its vertices $v_{i}$, facets $\mathcal{F}_{i}$, and normal vectors $h_{i}$.

(vii) The vectors $\left\{v_{1}-v_{0}, \ldots, v_{n}-v_{0}\right\}$ are a basis for $\mathbb{R}^{n}$.

(viii) The vectors $\left\{h_{1}, \ldots, h_{n}\right\}$ are a basis for $\mathbb{R}^{n}$.

(ix) There exist $\gamma_{1}>0, \ldots, \gamma_{n}>0$ such that $h_{0}=-\gamma_{1} h_{1}-\cdots-\gamma_{n} h_{n}$.

An $n$-dimensional polytope is the convex hull of a finite set of points in $\mathbb{R}^{n}[26]$. In particular, let $\left\{v_{1}, \cdots, v_{p}\right\}$ be a set of points in $\mathbb{R}^{n}$, where $p>n$, and suppose that $\left\{v_{1}, \cdots, v_{p}\right\}$ contains $(n+1)$ affinely independent points. We define the $n$-dimensional polytope

$$
\mathcal{P}:=\operatorname{co}\left\{v_{1}, \cdots, v_{p}\right\}
$$

Clearly, a simplex is a special case of polytopes in which $p=n+1$. Figure 2.2 shows a polytope in $\mathbb{R}^{2}$. A face of $\mathcal{P}$ is any sub-polytope of $\mathcal{P}$ which makes up its boundary. The polytope $\mathcal{P}$ itself is considered as a trivial face, and all other faces (of dimension less than $n$ ) are called proper faces. An edge of $\mathcal{P}$ is a 1-dimensional face of $\mathcal{P}$. A facet of $\mathcal{P}$ is an $(n-1)$-dimensional face of $\mathcal{P}$. We denote the facets of $\mathcal{P}$ by $\mathcal{F}_{0}, \cdots, \mathcal{F}_{r}$. Let $h_{i}$ denote the unit normal vector to $\mathcal{F}_{i}$ pointing outside $\mathcal{P}$. An implicit description of $\mathcal{P}$ can be obtained using the normal vectors. Precisely, there exist $\alpha_{1}, \cdots, \alpha_{r}$ such that

$$
\mathcal{P}=\left\{x \in \mathbb{R}^{n} \mid h_{i} \cdot x \leq \alpha_{i}, \forall i \in\{0, \cdots, r\}\right\}
$$




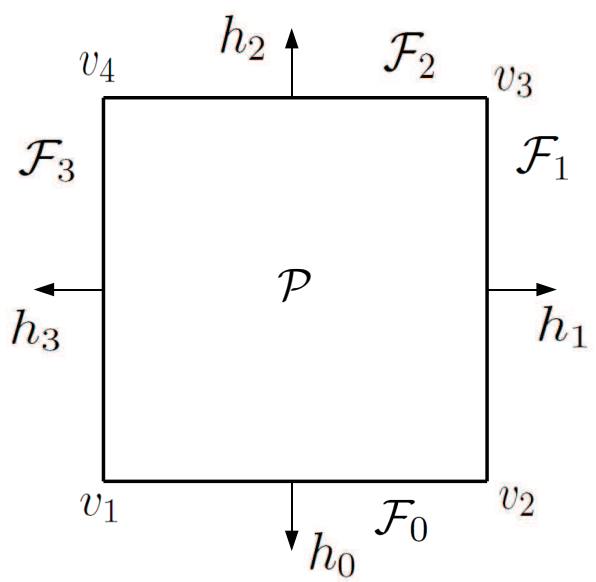

Figure 2.2: A 2D polytope $\mathcal{P}$ with its vertices $v_{i}$, facets $\mathcal{F}_{i}$ and normal vectors $h_{i}$.

In the following part, we review special types of polytopes. First, a simplicial polytope is a polytope whose proper faces are simplices. Second, we review generic polytopes [27]. A set of $p>n$ points in $\mathbb{R}^{n}$ are in general position if any $(n+1)$ points of them are affinely independent (form an $n$-dimensional simplex). A generic polytope is the convex hull of a set of points in general position in $\mathbb{R}^{n}$. For a generic polytope, all proper faces are simplices. Notice that any generic polytope is simplicial, while the converse is not true. Third, an $n$-dimensional polytope $\mathcal{P}$ is said to be simple if each $k$-dimensional face of $\mathcal{P}$ is contained in exactly $n-k$ facets.

Remark 2.1. If $\mathcal{P}$ is an $n$-dimensional simple polytope, then $\mathcal{P}$ has the following properties [26]:

(i) Each vertex of $\mathcal{P}$ is contained in exactly $n$ edges.

(ii) Let $\mathcal{F}$ be a facet of $\mathcal{P}$ and $v$ a vertex of $\mathcal{P}$ in $\mathcal{F}$. Then there are exactly $n-1$ edges in $\mathcal{F}$ containing $v$.

\subsection{Placing Triangulation}

In this section we describe a method to triangulate a polytopic space with respect to another space. We first provide a nominal definition for a triangulation. 
Definition $2.1([23])$. A triangulation $\mathbb{T}$ of an $n$-dimensional polytope $\mathcal{P}$ is a finite collection $n$-dimensional simplices $\mathcal{S}^{1}, \ldots, \mathcal{S}^{L}$ such that: (1) The vertices of each $\mathcal{S}^{i}$ are drawn from the vertices of $\mathcal{P}$ (though not every vertex of $\mathcal{P}$ need be used); (2) $\mathcal{P}=\cup_{i}^{L} \mathcal{S}^{i}$; (3) For all $i$, $j \in\{1, \ldots, L\}$ with $i \neq j$, then $\mathcal{S}^{i} \cap \mathcal{S}^{j}$ is a common (possibly empty) face of $\mathcal{S}^{i}$ and $\mathcal{S}^{j}$.

Let $\mathcal{P}$ be a polytope and let $\mathcal{O}$ be an affine space of dimension less than $n$. We assume $\mathcal{P} \cap \mathcal{O}$ is a polytope with vertices $V_{\mathcal{O}}:=\left\{o_{1}, \ldots, o_{r}\right\}$. We want to triangulate $\mathcal{P}$ with respect to $\mathcal{O}$.

First, we define an ordered point set $V:=\left\{v_{1}, \ldots, v_{p}\right\}$ such that $\mathcal{P}=\operatorname{co}(V)$ and the first $r$ points of $V$ are $V_{\mathcal{O}}$. Note that not every element of $V_{\mathcal{O}}$ need be a vertex of $\mathcal{P}$. Now we propose a triangulation of $\mathcal{P}$ which will have the feature that $\mathcal{O}$ can only lie in lower dimensional faces of simplices of the triangulation. We use a standard procedure called the placing triangulation (see $[23,22])$. To describe this triangulation method we need a few definitions.

Suppose $V$ is a finite set of points such that $\mathcal{P}=\operatorname{co}(V)$ is an $n$-dimensional polytope. A subdivision of $V$ is a finite collection $\mathbb{S}=\left\{\mathcal{P}_{1}, \ldots, \mathcal{P}_{q}\right\}$ of $n$-dimensional polytopes such that: (1) The vertices of each $\mathcal{P}_{i}$ are drawn from $V$ (though not every point in $V$ need be used); (2) $\mathcal{P}=\cup_{i} \mathcal{P}_{i}$; (3) If $i \neq j$, then $\mathcal{P}_{i} \cap \mathcal{P}_{j}$ is a common (possibly empty) face of the boundaries of $\mathcal{P}_{i}$ and $\mathcal{P}_{j}$.

Definition 2.2. Let $x \in \mathbb{R}^{n}, \mathcal{P}$ an $n$-dimensional polytope, and $\mathcal{F}$ a facet of $\mathcal{P}$. The hyperplane $\mathcal{H}=\operatorname{aff}(\mathcal{F})$ defines an open half-space containing $\operatorname{int}(\mathcal{P})$. If $x$ is contained in the opposite open half-space, then $\mathcal{F}$ is said to be visible from $x$. (If $\mathcal{P}$ is a $k$-dimensional polytope in $\mathbb{R}^{n}$ with $k<n$ and $x \in \operatorname{aff}(\mathcal{P})$, then the ambient space is viewed to be aff $(\mathcal{P})$.)

Now we can describe what it means to place a vertex. Let $\mathbb{S}=\left\{\mathcal{P}_{1}, \ldots, \mathcal{P}_{q}\right\}$ be a subdivision of $V$ and $v \in \mathbb{R}^{n}$ such that $v \notin V$.

Definition 2.3. The subdivision $\mathbb{T}$ of $V \cup\{v\}$ that results from placing $v$ is obtained as follows:

1. If $v \notin \operatorname{aff}(V)$, then for each $\mathcal{P}_{i} \in \mathbb{S}$, include $\operatorname{co}\left(\mathcal{P}_{i} \cup\{v\}\right)$ in $\mathbb{T}$.

2. If $v \in \operatorname{aff}(V)$, then for each $\mathcal{P}_{i} \in \mathbb{S}, \mathcal{P}_{i} \in \mathbb{T}$ and if $\mathcal{F}$ is a facet of $\mathcal{P}_{i}$ that is contained in a facet of $\operatorname{co}(V)$ visible from $v$, then $\operatorname{co}(\mathcal{F} \cup\{v\}) \in \mathbb{T}$. 


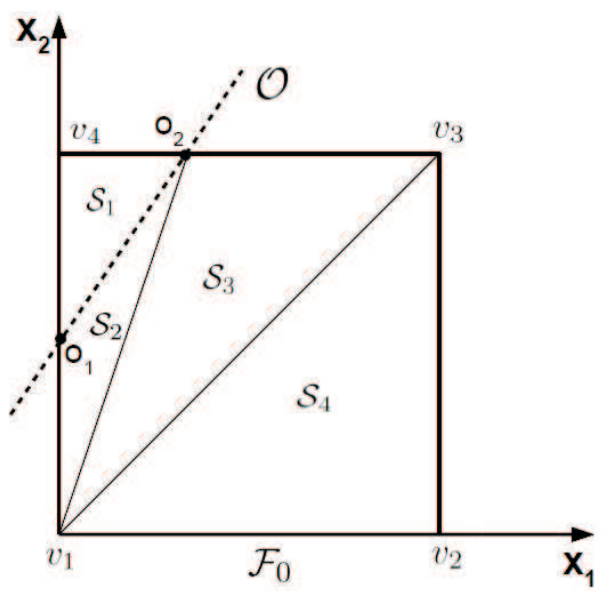

Figure 2.3: A sample triangulation of $\mathcal{P}$ with respect to the set $\mathcal{O}$ [2].

Theorem 2.1. [23] Suppose $V$ is a finite set of points such that $V_{\mathcal{O}} \subset V$ and $\mathcal{P}=\operatorname{co}(V)$ is an $n$-dimensional polytope. If the points of $V$ are ordered such that $\left\{o_{1}, \ldots, o_{r}\right\}$, the vertices of $\mathcal{P} \cap \mathcal{O}$, are listed first and if $\mathbb{T}$ is the subdivision obtained by placing the points of $V$ in order, then $\mathbb{T}$ is a triangulation of $V$ such that for every $n$-dimensional simplex $\mathcal{S} \in \mathbb{T}$, $\operatorname{int}(\mathcal{S}) \cap \mathcal{O}=\emptyset$ and if $\mathcal{S} \cap \mathcal{O} \neq \emptyset$, then $\mathcal{S} \cap \mathcal{O}$ is a face of $\mathcal{S}$.

Figure 2.3 depicts a sample triangulation of $\mathcal{P}$ with respect to the set $\mathcal{O}$. In particular, for each $\mathcal{S}_{i}, i=1,2,3,4$, the set $\mathcal{O} \cap \mathcal{S}_{i}$ is a $\kappa$-dimensional face of $\mathcal{S}_{i}$, where $0 \leq \kappa \leq n$. 


\section{Chapter 3}

\section{Reach Control Problem}

This thesis studies the reach control problem on simplices. The problem is for trajectories of an affine system defined on a simplex to exit a prespecified facet of the simplex in finite time without first leaving the simplex. In this chapter we explore basic principles which shape the features of the problem. These principles are derived from the geometry of the simplex and from convexity properties of affine systems. Most of the proofs in this chapter are suppressed since these results have appeared in previous theses or papers. Those proofs that are included will be highlighted or referenced in later chapters.

\subsection{Reach Control Problem}

We study an $n$-dimensional simplex defined by

$$
\mathcal{S}:=\operatorname{co}\left\{v_{0}, \ldots, v_{n}\right\}
$$

with vertices $\left\{v_{0}, \ldots, v_{n} \mid v_{i} \in \mathbb{R}^{n}\right\}$ that are affinely independent. Define the vertex set

$$
V:=\left\{v_{0}, \ldots, v_{n}\right\}
$$

We denote the $(n-1)$-dimensional facets by $\mathcal{F}_{0}, \ldots, \mathcal{F}_{n}$, where the index of each facet is

determined by the vertex it does not contain. Let $h_{j}, j=0, \ldots, n$ be the unit normal vector to each facet $\mathcal{F}_{j}$ pointing outside of the simplex. Facet $\mathcal{F}_{0}$ is called the exit facet of $\mathcal{S}$. Define the 


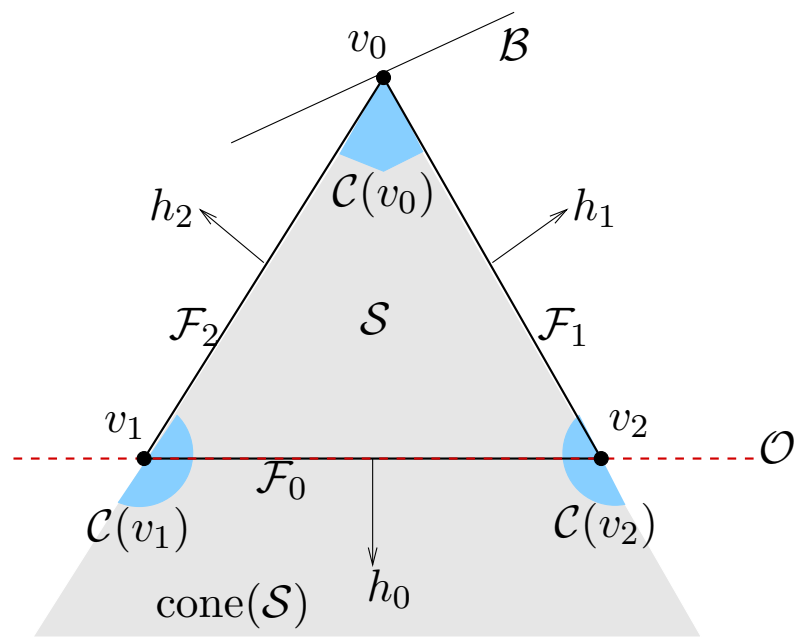

Figure 3.1: Notation for the reach control problem.

index set

$$
I:=\{1, \ldots, n\} .
$$

For $x \in \mathcal{S}$ defined the closed, convex cone

$$
\mathcal{C}(x):=\left\{y \in \mathbb{R}^{n} \mid h_{j} \cdot y \leq 0, j \in I, x \in \mathcal{F}_{j}\right\} .
$$

We introduce a more evocative notation

$$
\operatorname{cone}(\mathcal{S}):=\mathcal{C}\left(v_{0}\right)
$$

because $\mathcal{C}\left(v_{0}\right)$ is the tangent cone to $\mathcal{S}$ at $v_{0}$. Instead $\mathcal{C}\left(v_{i}\right)$ for $i \in I$ are not tangent cones to $\mathcal{S}$.

Consider the affine control system defined on $\mathcal{S}$ :

$$
\dot{x}=A x+a+B u, \quad x \in \mathcal{S},
$$

where $A \in \mathbb{R}^{n \times n}, a \in \mathbb{R}^{n}, B \in \mathbb{R}^{n \times m}$, and $\operatorname{rank}(B)=m$. Let $\mathcal{B}=\operatorname{Im}(B)$, the image of $B$. Define

$$
\mathcal{O}:=\left\{x \in \mathbb{R}^{n} \mid A x+a \in \mathcal{B}\right\}
$$


and

$$
\mathcal{G}:=\mathcal{S} \cap \mathcal{O}
$$

We also associate with $\mathcal{G}$ its vertex index set

$$
I_{\mathcal{G}}:=\left\{i: v_{i} \in V \cap \mathcal{G}\right\}
$$

Let $\phi_{u}\left(t, x_{0}\right)$ denote the trajectory of (3.1) starting at $x_{0}$ under a control $u$.

Example 3.1. Consider Figure 3.1 where we illustrate the notation in a 2D example. We have a full-dimensional simplex in $\mathbb{R}^{2}$ given by $\mathcal{S}=\operatorname{co}\left\{v_{0}, v_{1}, v_{2}\right\}$ with vertex set $V=\left\{v_{0}, v_{1}, v_{2}\right\}$ and $(n-1)$-dimensional facets $\mathcal{F}_{0}, \mathcal{F}_{1}$, and $\mathcal{F}_{2}$. Each facet $\mathcal{F}_{j}$ has an outward normal vector $h_{j}$. The only vertex not in facet $\mathcal{F}_{j}$ is vertex $v_{j} . \mathcal{F}_{0}$ is the exit facet. If we assume that $v_{0}=0$, then subspace $\mathcal{B}$ is shown passing through $v_{0}$. The set $\mathcal{O}$ is an affine space shown passing through $\mathcal{F}_{0}$. Notice in this case $\mathcal{G}=\mathcal{S} \cap \mathcal{O}=\operatorname{co}\left\{v_{1}, v_{2}\right\}$. The cone cone $(\mathcal{S})$ is the cone with apex at $v_{0}$ determined by $\mathcal{S}$. It is indicated in the figure as the shaded area. The cones $\mathcal{C}\left(v_{i}\right), i=0,1,2$ are depicted as darker shaded cones attached at each vertex. Of course, the apex of each $\mathcal{C}\left(v_{i}\right)$ is at the origin, but we depict it as being attached at the corresponding vertex $v_{i}$ since it will be used to describe allowable directions for the vector field at the vertices. Notice that the cones $\mathcal{C}\left(v_{1}\right)$ and $\mathcal{C}\left(v_{2}\right)$ are not tangent cones to $\mathcal{S}$ at $v_{1}$ and $v_{2}$, respectively, whereas $\mathcal{C}\left(v_{0}\right)$ is the tangent cone to $\mathcal{S}$ at $v_{0}$; hence the distinguished labeling of $\mathcal{C}\left(v_{0}\right)$ as cone $(\mathcal{S})$.

The problem we consider is one where closed-loop trajectories of (3.1) are driven out of $\mathcal{S}$ through the exit facet $\mathcal{F}_{0}$ only. For this, conditions are required that restrict trajectories from exiting the remaining facets $\mathcal{F}_{i}, i \in I$. In particular, it is said that the invariance conditions are solvable at vertex $v_{i} \in V$ if there exists $u_{i} \in \mathbb{R}^{m}$ such that

$$
A v_{i}+B u_{i}+a \in \mathcal{C}\left(v_{i}\right)
$$

Moreover, the invariance conditions are solvable if (3.4) is solvable at each $v_{i} \in V$. The inequalities (3.4) are called invariance conditions. They guarantee trajectories cannot exit from the facets $\mathcal{F}_{i}, i \in I$, and they are used to construct affine feedbacks [29]. For general state 


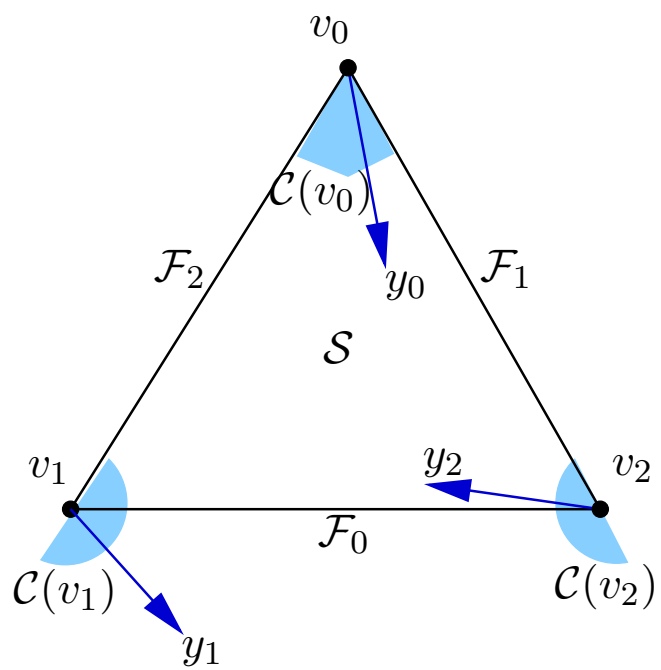

Figure 3.2: The invariance conditions state that $y_{i}:=A v_{i}+B u_{i}+a \in \mathcal{C}\left(v_{i}\right)$ for $i=0, \ldots, n$.

feedbacks, stronger conditions (also called invariance conditions) are needed. A state feedback $u=f(x)$ then satisfies the invariance conditions if for all $x \in \mathcal{S}$,

$$
A x+B f(x)+a \in \mathcal{C}(x) .
$$

Example 3.2. Consider Figure 3.2. Attached at each vertex is a velocity vector $y_{i}:=A v_{i}+$ $B u_{i}+a, i \in\{0\} \cup I$. The invariance conditions (3.4) require that $y_{i} \in \mathcal{C}\left(v_{i}\right)$, as illustrated. Notice that velocity vectors at $v_{i} \in \mathcal{F}_{0}$ may or may not point out of $\mathcal{S}$. If the control is an affine feedback $u=K x+g$ such that $u_{i}=K v_{i}+g$, then by convexity of the closed-loop vector field, (3.5) holds at every $x \in \mathcal{F}_{i}, i \in I$. If the input is a continuous state feedback $u=f(x)$, then invariance conditions for every $x \in \mathcal{F}_{i}, i \in I$, must be explicitly stated, since convexity is not guaranteed; hence (3.5).

Problem 3.1 (Reach Control Problem (RCP)). Consider system (3.1) defined on $\mathcal{S}$. Find a state feedback $u=f(x)$ such that for every $x \in \mathcal{S}$ there exist $T \geq 0$ and $\gamma>0$ such that $\phi_{u}(t, x) \in \mathcal{S}$ for all $t \in[0, T], \phi_{u}(T, x) \in \mathcal{F}_{0}$, and $\phi_{u}(t, x) \notin \mathcal{S}$ for all $t \in(T, T+\gamma)$.

In the sequel we will use the shorthand notation $\mathcal{S} \stackrel{\mathcal{S}}{\longrightarrow} \mathcal{F}_{0}$ to denote that (i)-(iii) of Problem 3.1 hold under some control law.

We now focus our attention on the set $\mathcal{O}$, which is critical in RCP. The most important 


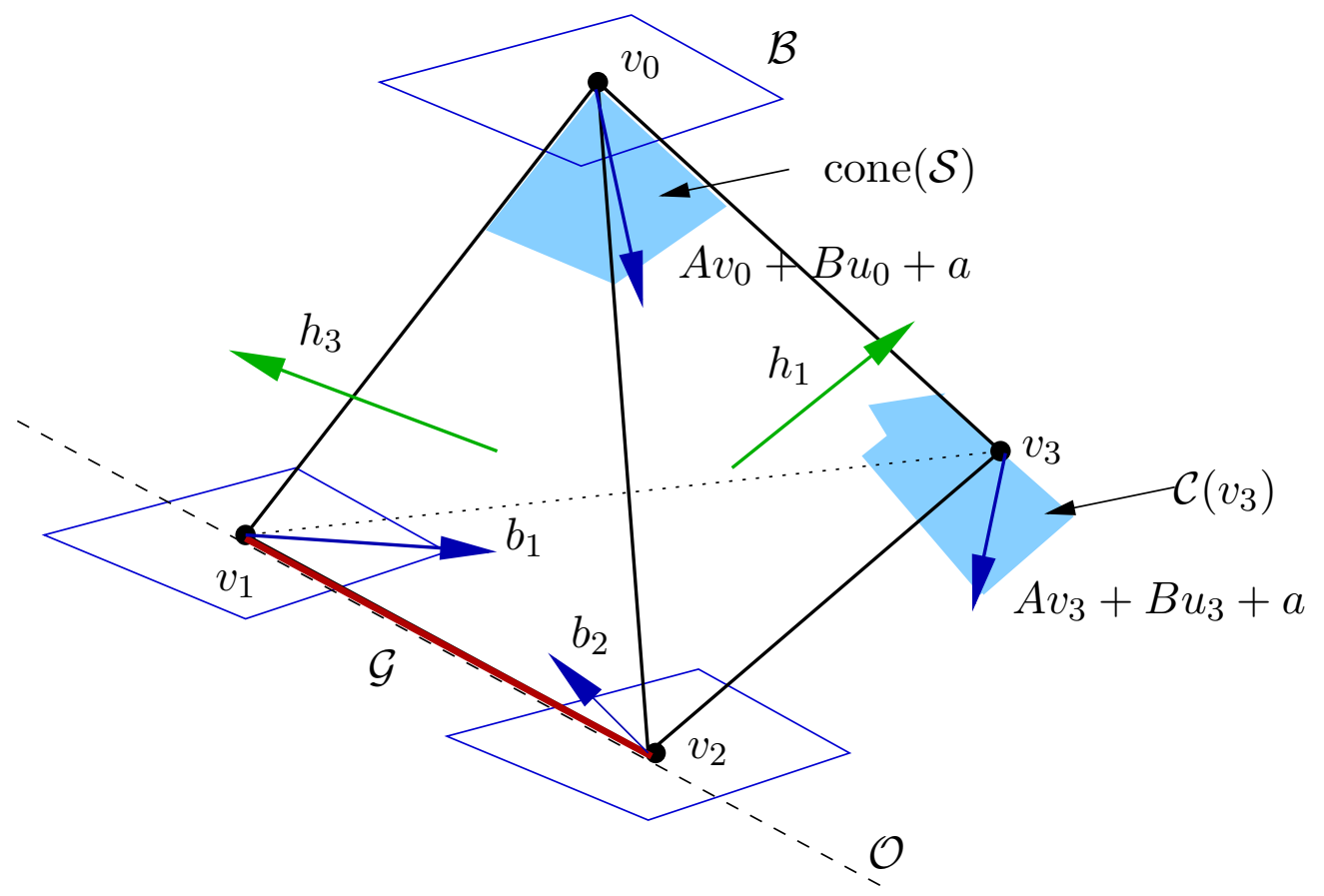

Figure 3.3: Geometric constructs for the reach control problem.

observation is that the vector field $A x+a+B u$ can vanish at any $x \in \mathcal{O}$ for an appropriate choice of $u \in \mathbb{R}^{m}$, so $\mathcal{O}$ is interpreted as the set of all possible equilibrium points of (3.1). Thus, if $x_{0}$ is an equilibrium of (3.1) under feedback control, then $x_{0} \in \mathcal{O}$. Similarly the set of possible equilibrium points of (3.1) on $\mathcal{S}$ is given by $\mathcal{G}:=\mathcal{S} \cap \mathcal{O}$.

The following lemma provides a characterization of $\mathcal{O}$.

\section{Lemma 3.1.}

(i) If $\operatorname{Im}(A) \subseteq \mathcal{B}$ and $a \notin \mathcal{B}$, then $\mathcal{O}=\emptyset$;

(ii) If $\operatorname{Im}(A) \subseteq \mathcal{B}$ and $a \in \mathcal{B}$, then $\mathcal{O}=\mathbb{R}^{n}$;

(iii) Otherwise, $\mathcal{O}$ is an affine space with $m \leq \operatorname{dim}(\mathcal{O})<n$.

Example 3.3. We conclude this section by giving a second example of the geometric constructs required for RCP for the the case $n=3$ and $m=2$. Consider Figure 3.3. We have a simplex $\mathcal{S}$ with normal vectors $h_{i}$ to each facet $\mathcal{F}_{i}$. Depicted by a shaded section is cone $(\mathcal{S})$, the tangent cone at $v_{0}$. The space $\mathcal{B}$ is copied to $v_{0}$, and in this view we see that $\mathcal{B} \cap \operatorname{cone}(\mathcal{S})=\mathbf{0}$. That is, $\mathcal{B}$ does not "dip" into the tangent cone at $v_{0}$. This geometric feature will be further analyzed 
in ensuing chapters. The affine set $\mathcal{O}$ intersects $\mathcal{S}$ along the face $\overline{v_{1} v_{2}}$, and this forms $\mathcal{G}$. It is interpreted as the set of possible equilibria of the system. We know that in $\mathcal{G}$, the only velocity vectors available to the closed loop system are vectors in $\mathcal{B}$. This is depicted by placing copies of $\mathcal{B}$ at each of the vertices of $\mathcal{G}$. Two velocity vectors $b_{1}$ and $b_{2}$ are shown, and these clearly satisfy the invariance conditions at $v_{1}$ and $v_{2}$, respectively. At vertices not in $\mathcal{G}$, the drift term $A x+a$ becomes relevant, and the figure depicts closed-loop velocity vectors at $v_{0}, v_{3} \notin \mathcal{G}$ which satisfy their respective invariance conditions. The invariance conditions can be interpreted in terms of the cones $\mathcal{C}\left(v_{i}\right)$. Consider vertex $v_{3}$ where $\mathcal{C}\left(v_{3}\right)$ is depicted by a shaded region. This cone is shaped like an open book whose spine is parallel to the face $\overline{v_{0} v_{3}}$ and whose cover and back cover lie in $\mathcal{F}_{2}$ and $\mathcal{F}_{1}$, respectively. The invariance condition at $v_{3}$ is satisfied if the closed-loop velocity vector $A v_{3}+B u_{3}+a$ lies in $\mathcal{C}\left(v_{3}\right)$.

\subsection{Affine Systems}

In this section well-known properties of affine systems on compact, convex sets are presented for the case where there is no control input. First, results regarding the relationship between positively invariant sets, existence of equilibria, and trajectories leaving the set in finite time are discussed. Second, the way in which trajectories exit a compact, convex set when invariance conditions such as (3.4) hold is shown. Proofs in this section are also not included because the results follow from well-known arguments in convex analysis.

Let $\mathcal{P} \subset \mathbb{R}^{n}$ be a compact, convex set and consider the affine system defined on $\mathcal{P}$ :

$$
\dot{x}=A x+a, \quad x \in \mathcal{P},
$$

where $A \in \mathbb{R}^{n \times n}$ and $a \in \mathbb{R}^{n}$. Let $\phi\left(t, x_{0}\right)$ denote the unique trajectory of (3.6) starting from $x_{0} \in \mathcal{P}$.

Lemma 3.2. Consider the affine system (3.6) defined on a compact, convex set $\mathcal{P}$. Suppose there exists $x_{0} \in \mathcal{P}$ such that $\phi\left(t, x_{0}\right) \in \mathcal{P}$ for all $t \geq 0$. Then the set

$$
\Phi:=\operatorname{co}\left\{\phi\left(t, x_{0}\right) \mid t \geq 0\right\} \subset \mathcal{P}
$$


is a positively invariant set. Moreover, $\Phi$ contains an equilibrium of (3.6), i.e. there exists $\bar{x} \in \Phi$ such that $A \bar{x}+a=0$.

Lemma 3.3. Consider the affine system (3.6) defined on a compact, convex set $\mathcal{P}$. We have $A x+a \neq 0$ for all $x \in \mathcal{P}$ if and only if there exists $\xi \in \mathbb{R}^{n}$ such that

$$
\xi \cdot(A x+a)<0, \quad x \in \mathcal{P} .
$$

Equation (3.7) is called a flow condition for system (3.6) on $\mathcal{P}$. A consequence of the existence of a flow condition on a compact, convex set is that all trajectories of (3.6) originating in the set eventually leave it.

Lemma 3.4. Consider the affine system (3.6) defined on a compact, convex set $\mathcal{P}$. Suppose that for all $x \in \mathcal{P}, A x+a \neq 0$. Then for each $x_{0} \in \mathcal{P}$, the trajectory starting at $x_{0}$ eventually leaves $\mathcal{P}$, i.e. there exists $t_{1}>0$ such that $\phi\left(t_{1}, x_{0}\right) \notin \mathcal{P}$.

The existence of a flow condition is also related to the set $\mathcal{O}$ for an affine control system. To see this, consider again the control system (3.1). The next result shows that a flow condition naturally arises on any compact, convex set that does not intersect $\mathcal{O}$. Importantly, the control input plays no role and, moreover, the origin of the vector $\xi$ can be made explicit.

Lemma 3.5. Consider the affine control system (3.1) defined on a compact, convex set $\mathcal{P}$. If $\mathcal{P} \cap \mathcal{O}=\emptyset$, then there exists $\xi \in \operatorname{Ker} B^{T}$ such that

$$
\xi \cdot(A x+a)<0, \quad \forall x \in \mathcal{P} .
$$

Lemma 3.5 allows us to return to Lemma 3.2 and make a statement about trajectories that do not exit $\mathcal{P}$ under an open-loop control for the control system (3.1). Informally, if a trajectory of an affine control system does not leave $\mathcal{P}$, it is because it encircles $\mathcal{P} \cap \mathcal{O}$, approaches $\mathcal{P} \cap \mathcal{O}$, or remains on $\mathcal{P} \cap \mathcal{O}$.

Lemma 3.6. Consider the affine control system (3.1) defined on a compact, convex set $\mathcal{P}$. Suppose there exists $x_{0} \in \mathcal{P}$ and an open-loop control $\mu(t)$ such that the (unique) solution 
$\phi_{\mu}\left(t, x_{0}\right)$ satisfies $\phi_{\mu}\left(t, x_{0}\right) \in \mathcal{P}$ for all $t \geq 0$. Then the set

$$
\Phi:=\operatorname{co}\left\{\phi\left(t, x_{0}\right) \mid t \geq 0\right\} \subset \mathcal{P}
$$

satisfies

$$
\bar{\Phi} \cap \mathcal{O} \neq \emptyset
$$

Finally, a result is presented that examines the way that trajectories of an affine system exit a compact, convex set when invariance conditions such as (3.4) or (3.5) hold.

Lemma 3.7. Consider the affine system (3.6) defined on a compact, convex set $\mathcal{P}$. Suppose additionally that $\mathcal{P}$ is a polytope with facets $\left\{\mathcal{F}_{0}, \ldots, \mathcal{F}_{k}\right\}$. Let $h_{i}$ be the outward normal vector of $\mathcal{F}_{i}$. Suppose that for some facet $\mathcal{F}_{i}$ the following conditions hold:

$$
h_{i} \cdot(A x+a) \leq 0, \quad \forall x \in \mathcal{F}_{i}
$$

Then all trajectories originating in $\mathcal{P}$ that leave $\mathcal{P}$ do so via a facet $\mathcal{F}_{j}, j \neq i$.

The next result shows that for affine systems, if trajectories exit from the proper exit facet, then the invariance conditions hold.

Lemma 3.8. Consider the affine system (3.6) defined on a compact, convex set $\mathcal{P}$. Suppose additionally that $\mathcal{P}$ is a polytope with facets $\left\{\mathcal{F}_{0}, \ldots, \mathcal{F}_{k}\right\}$. Let $h_{i}$ be the outward normal vector of $\mathcal{F}_{i}$. Suppose that $\mathcal{P} \stackrel{\mathcal{P}}{\longrightarrow} \mathcal{F}_{0}$. Then

$$
h_{i} \cdot(A x+a) \leq 0, \quad \forall x \in \mathcal{F}_{i}, i=1, \ldots, k
$$

\subsection{Affine Feedback}

In this section we present results from the literature [29]-[31], in which the properties of affine systems given in the previous section are used to produce a solution of RCP by affine feedback. The solution relies on a control synthesis procedure described next.

Lemma 3.9 ([29]). Consider two sets of points $V=\left\{v_{0}, \ldots, v_{n} \mid v_{i} \in \mathbb{R}^{n}\right\}$ and $\left\{u_{0}, \ldots, u_{n} \mid u_{i} \in\right.$ $\left.\mathbb{R}^{m}\right\}$. Suppose $V$ is affinely independent. Then there exist unique matrices $K \in \mathbb{R}^{m \times n}$ and 
$g \in \mathbb{R}^{m}$ such that

$$
u_{i}=K v_{i}+g, \quad i \in\{0, \ldots, n\} .
$$

Proof. We must show there exist matrices $K$ and $g$ such that

$$
\left[\begin{array}{ll}
v_{0}^{T} & 1 \\
\vdots & \vdots \\
v_{n}^{T} & 1
\end{array}\right]\left[\begin{array}{l}
K^{T} \\
g^{T}
\end{array}\right]=\left[\begin{array}{l}
u_{0}^{T} \\
\vdots \\
u_{n}^{T}
\end{array}\right]
$$

If the $(n+1) \times(n+1)$ left-hand matrix is full rank, then multiplying by its inverse yields the unique solutions $K$ and $g$. However,

$$
\operatorname{rank}\left[\begin{array}{ll}
v_{0}^{T} & 1 \\
\vdots & \\
v_{n}^{T} & 1
\end{array}\right]=1+\operatorname{rank}\left[\begin{array}{l}
v_{1}^{T}-v_{0}^{T} \\
v_{2}^{T}-v_{0}^{T} \\
\vdots \\
v_{n}^{T}-v_{0}^{T}
\end{array}\right]=1+n .
$$

The last equality follows since the points $\left\{v_{0}, \ldots, v_{n}\right\}$ are affinely independent if and only if $\left\{v_{1}-v_{0}, \ldots, v_{n}-v_{0}\right\}$ are linearly independent.

Using the results in Section 3.2 and the synthesis procedure of Lemma 3.9, the necessary and sufficient conditions for solvability of RCP by affine feedback are given in the following theorem.

Theorem 3.1 ([30, 31]). Given the system (3.1) and an affine feedback $u(x)=K x+g$, where $K \in \mathbb{R}^{m \times n}, g \in \mathbb{R}^{m}$, and $u_{0}=u\left(v_{0}\right), \ldots, u_{n}=u\left(v_{n}\right)$, the closed-loop system satisfies $\mathcal{S} \stackrel{\mathcal{S}}{\longrightarrow} \mathcal{F}_{0}$ if and only if

(a) The invariance conditions (3.4) hold.

(b) There is no equilibrium in $\mathcal{S}$.

Proof.

$(\Rightarrow)$ If $\mathcal{S} \stackrel{\mathcal{S}}{\longrightarrow} \mathcal{F}_{0}$ by affine feedback, then clearly the closed-loop system does not have equilibria 
in $\mathcal{S}$, for otherwise trajectories starting at an equilibrium would not leave $\mathcal{S}$. The invariance conditions (3.4) hold by Lemma 3.8.

$(\Leftarrow)$ By assumption, for vertex set $V$ there exist inputs $\left\{u_{0}, \ldots, u_{n}\right\}$ satisfying the invariance conditions (3.4). Invoking Lemma 3.9, there exists an affine feedback $u=K x+g$ such that the invariance conditions are satisfied at the vertices. The resulting closed-loop system is

$$
\dot{x}=(A+B K) x+(B g+a)=\tilde{A} x+\tilde{a} .
$$

By assumption, $\tilde{A} x+\tilde{a} \neq 0$ for all $x \in \mathcal{S}$, so by Lemma 3.4 , all trajectories leave $\mathcal{S}$ in finite time. From (3.4),

$$
h_{j} \cdot\left(\tilde{A} v_{i}+\tilde{a}\right) \leq 0, \quad i \in\{0, \ldots, n\}, \quad j \in I_{i} .
$$

By convexity

$$
h_{i} \cdot(\tilde{A} x+\tilde{a}) \leq 0, \quad \forall x \in \mathcal{F}_{i}, \quad i \in I
$$

By Lemma 3.7 trajectories cannot leave $\mathcal{S}$ via $\mathcal{F}_{1}, \ldots, \mathcal{F}_{n}$. This proves condition (i) of RCP. For condition (ii), since $\|\tilde{A} x+\tilde{a}\| \neq 0$ for all $x \in \mathcal{S}, \mathcal{S}$ is compact, and $x \mapsto\|\tilde{A} x+\tilde{a}\|$ is continuous, there exists $\varepsilon>0$ such that $\|\tilde{A} x+\tilde{a}\|>\varepsilon$ for all $x \in \mathcal{S}$.

Theorem 3.1 gives conditions for the solvability of RCP which are primarily of theoretical interest. In practice, these conditions do not realize a synthesis procedure, for one cannot guarantee that a proposed affine feedback satisfying the invariance conditions does not place closed-loop equilibria in $\mathcal{S}$. On the other hand, existence of a flow condition guarantees there are no equilibria in $\mathcal{S}$ by Lemma 3.3, so one can replace the requirement of no closed-loop equilibria with existence of a flow condition. This observation leads to an alternative set of necessary and sufficient conditions for solvability of RCP.

Theorem $3.2([31]) . \mathcal{S} \stackrel{\mathcal{S}}{\longrightarrow} \mathcal{F}_{0}$ by affine feedback if and only if there exist $u_{0}, \ldots, u_{n} \in \mathbb{R}^{m}$ and $\xi \in \mathbb{R}^{n}$ such that

(a) The invariance conditions (3.4) hold. 
(b) The flow condition holds: $\quad \xi \cdot\left(A v_{i}+B u_{i}+a\right)<0, \quad i \in\{0, \ldots, n\}$.

Theorem 3.2 can be viewed as a computational solution to the problem. If the invariance and flow conditions can be solved simultaneously for the unknowns $\xi \in \mathbb{R}^{n}$ and $u_{i} \in \mathbb{R}^{m}$, then an affine feedback can be constructed by the procedure of Lemma 3.9. Unfortunately, this approach relies on solving bilinear inequalities, which are known to be $N P$-hard [34].

We now present results for a variation of Problem 3.1 whereby it is desired to keep trajectories within a simplex $\mathcal{S}$ for all time, and to stabilize the system to a point $\bar{x} \in \mathcal{S}$. That is, a state feedback $u=f(x)$ is to be found such that:

(i) For every $x \in \mathcal{S}$ then $\phi_{u}(t, x) \in \mathcal{S}, t \geq 0$.

(ii) Given $\bar{x} \in \mathcal{S}$, solve (i) and achieve $\phi_{u}(t, x) \rightarrow \bar{x}$ as $t \rightarrow \infty$.

The solution to (i) amounts to the solvability of the invariance conditions where $I:=\{0, \ldots, n\}$ such that there is no exit facet.

Theorem 3.3 ([30]). For every $x \in \mathcal{S}, \phi_{u}(t, x) \in \mathcal{S}, t \geq 0$ by affine feedback if and only if there exist $u_{0}, \ldots, u_{n} \in \mathbb{R}^{m}$ such that $\forall i, j \in\{0, \ldots, n\}, i \neq j: h_{j}^{T} \cdot\left(A v_{i}+B u_{i}+a\right) \leq 0$.

The solution to (ii) additionally requires the geometric condition that equilibria may exist in $\mathcal{S}$, i.e. that $\mathcal{G}=\mathcal{S} \cap \mathcal{O} \neq \emptyset$, and that $\bar{x}$ lies on the set of possible equilibria. Moreover the closed-loop velocities at the vertices of $\mathcal{S}$ must span $\mathbb{R}^{n}$.

Theorem 3.4 ([30]). Let $\bar{x} \in \mathcal{S}$. Condition (i) is solved and $\phi_{u}(t, x) \rightarrow \bar{x}$ as $t \rightarrow \infty$ by affine feedback if and only if there exist $u_{0}, \ldots, u_{n} \in \mathbb{R}^{m}$ such that

(i) $\forall i, j \in\{0, \ldots, n\}, i \neq j: h_{j}^{T} \cdot\left(A v_{i}+B u_{i}+a\right) \leq 0$

(ii) $\bar{x} \in \mathcal{O}$

(iii) $\operatorname{span}\left(\left\{A v_{i}+B u_{i}+a \mid i=0, \ldots, n\right\}\right)=\mathbb{R}^{n}$.

\subsection{Triangulation and Affine Feedback}

In this section we assume a specific triangulation of the state space which makes possible the use of various results in the literature for such triangulations [33]. Specifically, the selected 
triangulation is attractive because it yields more geometric necessary and sufficient conditions for solvability of RCP. Note that a similar discussion of the material to come was presented in [3].

We retain our declaration of a simplex $\mathcal{S}=\operatorname{co}\left\{v_{0}, v_{1}, \ldots, v_{n}\right\}$ and the affine system defined on $\mathcal{S}$

$$
\dot{x}=A x+B u+a, \quad x \in \mathcal{S},
$$

where $A \in \mathbb{R}^{n \times n}, a \in \mathbb{R}^{n}, B \in \mathbb{R}^{n \times m}$, and $\operatorname{rank}(B)=m$.

We present first the geometric sufficient conditions for existence of affine feedbacks solving RCP with respect to the sets $\mathcal{O}$ and $\mathcal{G}$, defined in (3.2) and (3.3), respectively. We remark that the invariance conditions by themselves are generally not enough to produce a solution to RCP by affine feedback, as Theorem 3.1 indicates. However, there is one extreme case when the invariance conditions sufficiently solve the problem. This idea depends on combining Theorem 3.1 with the notion that equilibria may only appear on the set $\mathcal{O}$.

Theorem 3.5 ([33]). Suppose $\mathcal{G}=\emptyset$. If the invariance conditions are solvable, then $\mathcal{S} \stackrel{\mathcal{S}}{\longrightarrow} \mathcal{F}_{0}$ by affine feedback.

In general it is difficult to extend results such as Theorem 3.5. Instead, a preferred triangulation of the state space is used, presented in Section 2.3, which expedites the problem of obtaining geometric necessary and sufficient conditions for a solution to RCP. Imperative to the approach is the following assumption.

Assumption 3.1. Simplex $\mathcal{S}$ and system (3.13) satisfy the following condition: if $\mathcal{G} \neq \emptyset$, then $\mathcal{G}$ is a $\kappa$-dimensional face of $\mathcal{S}$, where $0 \leq \kappa \leq n$.

Remark 3.1. There are three possible forms for $\mathcal{O}$, and hence three possible approaches to a solution. If $\mathcal{O}=\emptyset$, then one applies Theorem 3.5. If $\mathcal{O}$ is the entire state space then it will be shown that there are easily derived necessary and sufficient conditions for solvability. The only interesting case is when $\mathcal{O}$ is a $\kappa$-dimensional affine subspace with $\kappa<n$. This case arises, for example, when $(A, B)$ is controllable, and then the placing triangulation can be applied.

With this result, several new sufficient conditions for existence of an affine feedback that will solve RCP have been shown to exist, which are presented below. In particular, the preferred 
triangulation of the state space with respect to $\mathcal{O}$ has been exploited to obtain such results. In particular, application of Lemma 3.5, along with additional conditions, has been found to achieve on $\mathcal{S}$ a flow condition satisfying Theorem 3.2.

Theorem 3.6 ([33]). Suppose Assumption 3.1 holds and $\mathcal{G} \neq \emptyset$. Suppose the following conditions hold.

1. The invariance conditions (3.4) are solvable.

2. $\mathcal{B} \cap \operatorname{cone}(\mathcal{S}) \neq \mathbf{0}$.

Then $\mathcal{S} \stackrel{\mathcal{S}}{\longrightarrow} \mathcal{F}_{0}$ by affine feedback.

Sufficient conditions for the existence of affine feedback are also feasible when $\mathcal{B} \cap \operatorname{cone}(\mathcal{S})=$ $\mathbf{0}$; this may only occur if $v_{0} \notin \mathcal{G}$. This relies on the idea that there are enough degrees of freedom in $\mathcal{B}$ with respect to $\mathcal{G}$. Consider then the following assumptions.

\section{Assumption 3.2.}

$(\mathrm{A} 1) \mathcal{G}=\operatorname{co}\left\{v_{1}, \ldots, v_{\kappa+1}\right\}$, with $0 \leq \kappa<m$.

(A2) $\mathcal{B} \cap \operatorname{cone}(\mathcal{S})=\mathbf{0}$.

(A3) There exists a linearly independent set $\left\{b_{i} \in \mathcal{B} \cap \mathcal{C}_{i} \mid i \in I_{\mathcal{G}}\right\}$.

The important new assumption is (A3) which says that $\mathcal{B}$ and $\mathcal{G}$ are arranged with respect to each other so that enough degrees of freedom exist in $\mathcal{B}$ both to span a $\kappa+1$-dimensional subspace of $\mathcal{B}$ and at the same time satisfy all the invariance conditions for the vertices of $\mathcal{G}$, indexed by $I_{\mathcal{G}}$. To achieve this we require $\kappa<m$.

Theorem 3.7 ([33]). Suppose Assumption 3.1 holds and $\mathcal{G}=\operatorname{co}\left\{v_{1}, \ldots, v_{\kappa+1}\right\}$, with $0 \leq \kappa<m$. Suppose the following conditions hold.

1. The invariance conditions (3.4) are solvable.

2. There exists a linearly independent set $\left\{b_{i} \in \mathcal{B} \cap \mathcal{C}_{i} \mid i \in I_{\mathcal{G}}\right\}$.

Then $\mathcal{S} \stackrel{\mathcal{S}}{\longrightarrow} \mathcal{F}_{0}$ by affine feedback. 


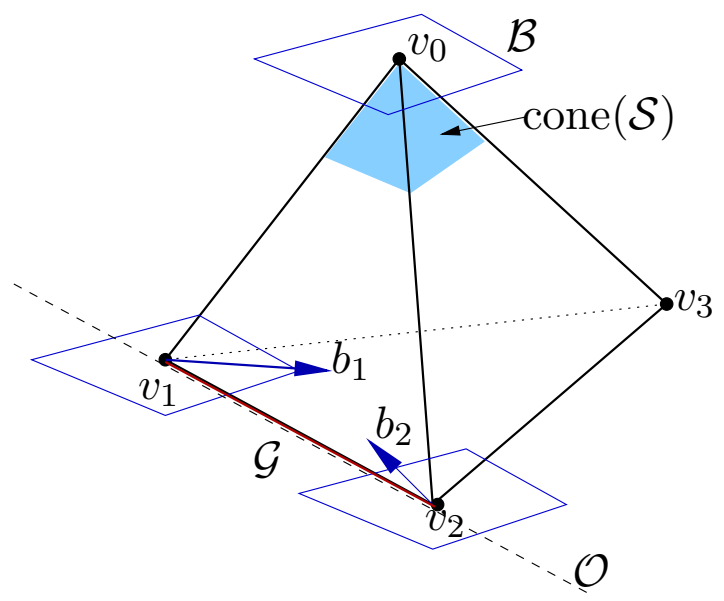

Figure 3.4: Continuous Assignment Along $\mathcal{G}$ of Vectors in $\mathcal{B}$.

Example 3.4. Consider the $2 \mathrm{D}$ simplex $\mathcal{S}=\operatorname{co}\left\{v_{0}, v_{1}, v_{2}\right\}$ with $v_{0}=(0,0), v_{1}=(1,2)$ and $v_{2}=(3,2)$. Consider the affine system on $\mathcal{S}$

$$
\dot{\xi}=\left[\begin{array}{rr}
-1 & 1 \\
1 & 0
\end{array}\right] \xi+\left[\begin{array}{l}
0 \\
1
\end{array}\right] u+\left[\begin{array}{l}
4 \\
2
\end{array}\right]
$$

The normal vectors of the facets of $\mathcal{S}$ are $h_{0}=(0,0.5), h_{1}=(0.5,-0.75)$ and $h_{2}=(-0.5,0.25)$. The objective is to solve the reach control problem on $\mathcal{S}$ with respect to exit facet $\mathcal{F}_{0}$. First, we calculate the set of possible equilibria $\mathcal{O}=\left\{\xi \in \mathbb{R}^{2} \mid \xi_{1}-\xi_{2}-4=0\right\}$. Then, we compute $\mathcal{G}=\mathcal{S} \cap \mathcal{O}=\emptyset$. Since no equilibria can exist on $\mathcal{S}$, then by Theorem 3.5, if the invariance conditions are solvable, then $\mathcal{S} \stackrel{\mathcal{S}}{\longrightarrow} \mathcal{F}_{0}$ by affine feedback. To that end, let $y_{i}:=A v_{i}+B u_{i}+a$, $i=0,1,2$. From (3.4) we have the following invariance conditions for $\mathcal{S}$ :

$$
\begin{array}{ll}
v_{0}: & h_{1} \cdot y_{0} \leq 0 \Longrightarrow(0.5,-0.75) \cdot\left(4,2+u_{0}\right) \leq 0 \Longrightarrow \frac{2}{3} \leq u_{0} \\
& h_{2} \cdot y_{0} \leq 0 \Longrightarrow(-0.5,0.25) \cdot\left(4,2+u_{0}\right) \leq 0 \Longrightarrow u_{0} \leq 6 \\
v_{1}: & h_{2} \cdot y_{1} \leq 0 \Longrightarrow(-0.5,0.25) \cdot\left(5,3+u_{1}\right) \leq 0 \Longrightarrow u_{1} \leq 7 \\
v_{2}: & h_{1} \cdot y_{2} \leq 0 \Longrightarrow(0.5,-0.75) \cdot\left(3,5+u_{2}\right) \leq 0 \Longrightarrow-3 \leq u_{2} .
\end{array}
$$

We choose $u_{0}=4, u_{1}=2$ and $u_{2}=-1$ as the control values, which satisfy the inequalities above. With the chosen controls and the $v_{i}$ we generate the feedback parameters $K$ and $g$ using 


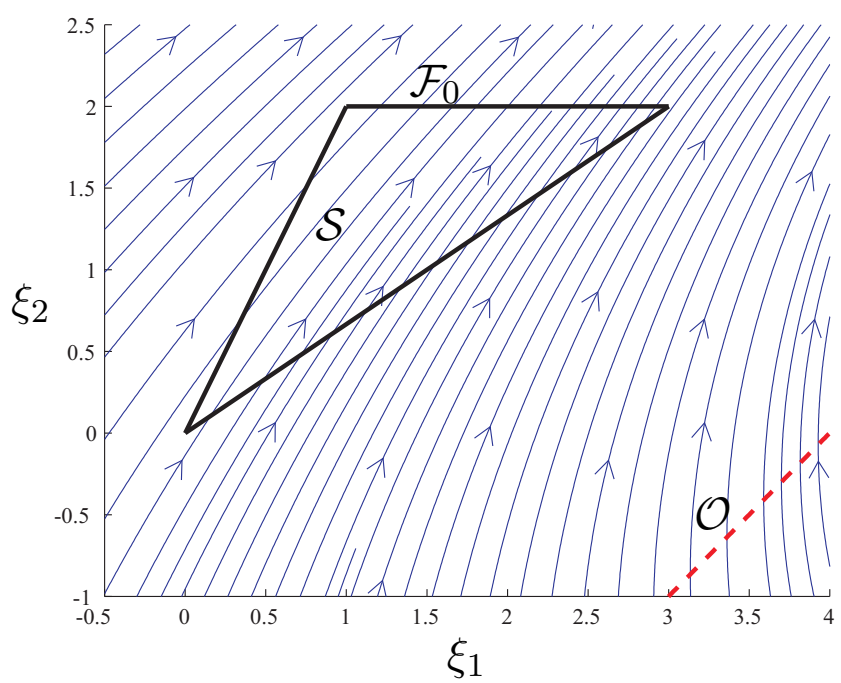

Figure 3.5: Closed-loop vector field on $\mathcal{S}$ for Example 3.4.

(3.10) and construct

$$
u=[-1.5-0.25] \xi+4, \quad \xi \in \mathcal{S}
$$

In Figure 3.5 the simplex $\mathcal{S}$ and the closed-loop vector field under $u$ are depicted. Clearly, for any initial condition in $\mathcal{S}$ trajectories are driven across $\mathcal{F}_{0}$ without crossing other facets.

\subsection{Reach Control on Polytopes}

In this thesis there will prove to be scenarios in which a solution to RCP using simplex methods fails to materialise, due to the restrictive nature of the simplex. In particular, the inherent structure of the system may render it difficult to guarantee that the invariance conditions hold in order to allow trajectories to pass through some facets but not others. In this instance we turn to polytopes, which are effectively a collection of simplices, for which a desired system behaviour may be achieved using a similar design methodology. The material to come skims the surface of the problem of RCP on polytopes which is dealt with in full in [2]. The reader is referred to [2] for an illustration of the proofs for the cited results, as well as an extensive comparison of simplex and polytope solutions. 
Consider an $n$-dimensional polytope

$$
\mathcal{P}:=\operatorname{co}\left\{v_{1}, \ldots, v_{p}\right\}
$$

with vertex set $V:=\left\{v_{1}, \ldots, v_{p}\right\}$ and facets $\mathcal{F}_{0}, \mathcal{F}_{1}, \ldots, \mathcal{F}_{r}$. Denote $\mathcal{F}_{0}$ the exit facet of $\mathcal{P}$, and $\mathcal{F}_{1}, \ldots, \mathcal{F}_{r}$ the restricted facets of $\mathcal{P}$. Let $h_{i}$ be the outward-pointing unit normal vector to each facet $\mathcal{F}_{i}$. Define the index sets $I_{P}:=\{1, \ldots, p\}, J:=\{1, \ldots, r\}$, and $J(x):=\left\{j \in J \mid x \in \mathcal{F}_{j}\right\}$. That is, $J(x)$ is the set of indices of the restricted facets in which $x$ is a point. As in Section 3.1 , for each $x \in \mathcal{P}$, define the closed, convex cone

$$
\mathcal{C}(x):=\left\{y \in \mathbb{R}^{n} \mid h_{j} \cdot y \leq 0, j \in J(x)\right\}
$$

Note that $\mathcal{F}_{0}$ never appears in this definition of $\mathcal{C}(x)$ since $\mathcal{F}_{0}$ is the exit facet. We consider the affine control system defined on $\mathcal{P}$ :

$$
\dot{x}=A x+B u+a, \quad x \in \mathcal{P},
$$

where $A \in \mathbb{R}^{n \times n}, a \in \mathbb{R}^{n}, B \in \mathbb{R}^{n \times m}$, and $\operatorname{rank}(B)=m$. Let $\phi_{u}\left(t, x_{0}\right)$ be the trajectory of (3.14) under a control law $u$ starting from $x_{0} \in \mathcal{P}$, and let $\mathcal{B}=\operatorname{Im}(B)$, the image of $B$. We are interested in studying reachability of the exit facet $\mathcal{F}_{0}$ from $\mathcal{P}$ by feedback control.

Also as in Section 3.1 define the set of possible equilibrium points of (3.1) on $\mathcal{P}$ by

$$
\mathcal{O}_{\mathcal{P}}:=\mathcal{P} \cap \mathcal{O},
$$

where $\mathcal{O}$ is defined in (3.2). Since $\mathcal{O}$ is an affine space, either $\mathcal{O}_{\mathcal{P}}=\emptyset$ or $\mathcal{O}_{\mathcal{P}}$ is a $\kappa$-dimensional polytope in $\mathcal{P}$. If $\mathcal{O}_{\mathcal{P}} \neq \emptyset$, we define the vertex set of $\mathcal{O}_{\mathcal{P}}$ to be $V_{\mathcal{O}}:=\left\{o_{1}, \ldots, o_{q}\right\}$, where $o_{i}$ are the vertices of $\mathcal{O}_{\mathcal{P}}$ (not necessarily vertices of $\mathcal{P}$ ). Also define the index set $I_{\mathcal{O}}:=\{1, \ldots, q\}$. We say $\mathbb{T}$ is a triangulation of $\mathcal{P}$ with respect to $\mathcal{O}$ if $\mathbb{T}$ is a triangulation of $\mathcal{P}$ such that $\mathcal{O}_{\mathcal{P}}$ is a union of simplices of the triangulation. We specify further criteria for the triangulation in order to obtain and harness specific geometric properties that are helpful in developing a solution to RCP. In particular, in Section 3.4 we used the assumption that $\mathcal{G}$ is a face of the simplex. We 
adopt the same type of assumption for polytopes.

Assumption 3.3. Polytope $\mathcal{P}$ and system (3.14) satisfy the following condition: $\mathcal{O}_{\mathcal{P}}$ is a $\kappa$-dimensional face of $\mathcal{P}$, where $0 \leq \kappa \leq n$. In particular,

$$
\mathcal{O}_{\mathcal{P}}=\operatorname{co}\left\{v_{1}, \ldots, v_{q},\right\}
$$

where $v_{i}$ is a vertex of $\mathcal{P}_{i}$.

The procedure of triangulating $\mathcal{P}$ in such a manner is detailed in Section 2.3. We now repeat the notion of the invariance conditions in the context of polytopes.

Definition 3.1. We say the invariance conditions are solvable if for each $v \in V$ there exists $u \in \mathbb{R}^{m}$ such that

$$
A v+B u+a \in \mathcal{C}(v)
$$

Equivalently,

$$
h_{j} \cdot(A v+B u+a) \leq 0, \quad j \in J(v) .
$$

Also define the following closed-loop velocity vectors associated with the set $\mathcal{O}_{\mathcal{P}}$ :

$$
b_{i}:=A o_{i}+B u\left(o_{i}\right)+a \in \mathcal{B} \cap \mathcal{C}\left(o_{i}\right), \quad i \in I_{\mathcal{O}} .
$$

We now present a variation of the reach control problem for polytopes which is called the Monotonic Reach Control Problem (MRCP).

Problem 3.2 (Monotonic Reach Control Problem (MRCP)). Consider system (3.14) defined on a convex polytope $\mathcal{P}$. Find a state feedback $u(x)$ such that for each initial condition $x_{0} \in \mathcal{P}$, there exist $T \geq 0$ and $\gamma>0$ such that

(i) $\phi_{u}\left(t, x_{0}\right)$ remains in $\mathcal{P}$ for all time $t \in[0, T] ; \phi_{u}\left(t, x_{0}\right)$ reaches $\mathcal{F}_{0}$ at time $T ; \phi_{u}\left(t, \xi_{0}\right)$ leaves $\mathcal{P}$ during an interval of time $t \in(T, T+\gamma)$.

(ii) There exists $\xi \in \mathbb{R}^{n}$ such that for all $x \in \mathcal{P}, \xi \cdot(A x+B u(x)+a)<0$.

Again, the goal is to construct a piecewise affine (PWA) state feedback law that will ensure that any closed-loop trajectory that starts in $\mathcal{P}$ may only exit the polytope through the exit 
facet $\mathcal{F}_{0}$. Condition (ii) is new with respect to Problem 3.1, and is called a flow condition. Moreover, the term "monotonic" refers to the evolution of trajectories through the polytope in a common sense with respect to consecutive, parallel hyperplanes characterized by the normal vector $\xi$. We write $\mathcal{P} \stackrel{\mathcal{P}}{\rightarrow} \mathcal{F}_{0}$ monotonically where conditions (i)-(ii) from above hold.

In general, numerical algorithms are required to determine the existence of $\xi$, however a set of geometric arguments may be made that connect the flow condition to the existence of equilibria on $\mathcal{P}$. In particular, the first result addresses the case where $\mathcal{O}_{\mathcal{P}}=\emptyset$, to which we refer the reader to Lemma 3.3. In effect, the lack of equilibria on $\mathcal{P}$ implies that trajectories must flow out of $\mathcal{P}$. Consequently, a result analogous to that of Theorem 3.1 for simplices follows, where the lack of equilibria simply requires the satisfaction of the invariance conditions for MRCP to be solved.

Theorem $3.8([2])$. Consider the system (3.14) defined on a polytope $\mathcal{P}$, and suppose $\mathcal{O}_{\mathcal{P}}=\emptyset$. Then $\mathcal{P} \stackrel{\mathcal{P}}{\rightarrow} \mathcal{F}_{0}$ if and only if the invariance conditions (3.16) are solvable.

Commonly, $\mathcal{O}_{\mathcal{P}} \neq \emptyset$, and thus a trivial argument guaranteeing no equilibria in $\mathcal{P}$ may not be made. However, additional geometric conditions associated with the closed-loop velocities at the vertices $V_{\mathcal{O}}$ (as a result of an affine feedback) can be used to determine when there are no equilibria in $\mathcal{P}$.

Theorem 3.9 ([2]). Consider the system (3.14) defined on a polytope $\mathcal{P}$. Let $\mathbb{T}$ be a triangulation of $\mathcal{P}$ with respect to $\mathcal{O}, u(x)$ be a piecewise affine feedback defined on $\mathbb{T}$, and $b_{i}$ be as in (3.17). If $0 \notin \operatorname{co}\left\{b_{1}, \ldots, b_{q}\right\}$, then the closed-loop system has no equilibrium in $\mathcal{P}$.

The condition above equivalently implies the lack of equilibria on $\mathcal{P}$ and the existence of a flow-like vector $\xi$ from (ii) of Problem 3.2. With these results in hand, the following theorem provides the necessary and sufficient conditions for a solution to MRCP as used in this thesis.

Theorem 3.10 ([2]). Consider the system (3.14) defined on $\mathcal{P}$ and suppose Assumption 3.3 holds. Then $\mathcal{P} \stackrel{\mathcal{P}}{\rightarrow} \mathcal{F}_{0}$ monotonically by continuous piecewise affine feedback if and only if

(i) The invariance conditions (3.16) hold.

(ii) There exists $\left\{b_{1}, \ldots, b_{q} \mid b_{i} \in \mathcal{B} \cap \mathcal{C}\left(v_{i}\right)\right\}$ such that $0 \notin \operatorname{co}\left\{b_{1}, \ldots, b_{q}\right\}$. 
Finally, the methodology for obtaining an affine feedback that solves MRCP is as follows. For an affine system defined on a polytope we first select control values at the vertices of $\mathcal{P}$ to satisfy the invariance conditions (3.16). Then the polytope is triangulated into a number of simplices $\mathcal{S}^{k}, k>1$ such that $\mathcal{O} \cap \mathcal{S}^{k}$ is a $\kappa$-dimensional face of $\mathcal{S}^{k}$, where $0 \leq \kappa \leq n$. A continuous affine feedback of the form $u=K x+g$ is generated for each $\mathcal{S}^{k} \subset \mathcal{P}$ by applying (3.10) with the $u_{i}$ and $v_{i}$ belonging to $\mathcal{S}^{k}$. A hybrid switching system is then constructed on $\mathcal{P}$ with feedback $u_{\mathcal{P}}(x)$ defined as

$$
u_{\mathcal{P}}(x):=K^{k} x+g^{k}, \quad x \in \mathcal{S}^{k} .
$$




\section{Chapter 4}

\section{Three-Link Manipulator}

In this thesis the dynamic systems being studied are serial robotic manipulators. In this chapter we introduce the manipulator system under consideration in the form of a planar three-link manipulator, which provides the minimum degrees of freedom necessary to realize the desired maneuver. The first section of this chapter provides the basic background material for the modelling of the manipulator. The second section explores the dynamics of the full system under consideration, with the objective of reducing its complexity to obtain linear state space models. These models will be used in the next chapter to derive a solution to the bouncing problem.

\subsection{Manipulator Modelling}

We begin this section by briefly highlighting from [5] and [35] the notation and mathematical tools that are necessary for the modelling of manipulators in general and later on the SPDM. In particular the concepts of coordinate frames and rotations are defined, as well as mathematical operators of interest.

In this thesis a coordinate frame in $\mathbb{R}^{n}$ is defined by an origin (a point in space) to which a set of $n$ standard basis vectors are attached, representing the coordinate axes (typically we will use $n=2,3$ ) [35]. Assuming $n=3$, a coordinate frame is denoted by $F_{i}$ or $o_{i} x_{i} y_{i} z_{i}$, referring to origin $o_{i}$ and coordinate axes $x_{i}, y_{i}, z_{i}$ oriented according to the right hand rule, see Figure 4.1. A vector $r \in \mathbb{R}^{n}$ is expressed in $F_{i}$ using a superscript $i$, i.e. $r^{i}$. When a base or reference 

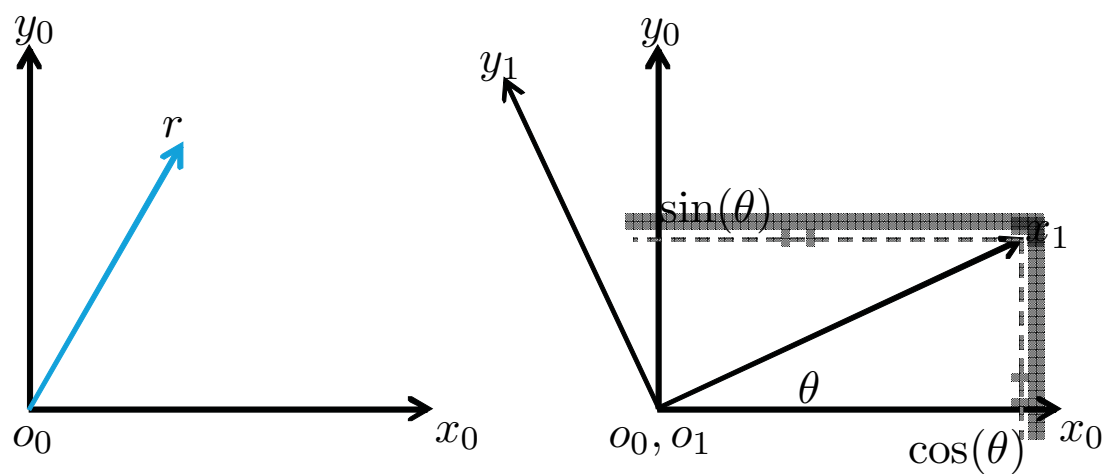

Figure 4.1: A vector $r$ in frame $F_{0}$ with coordinate axes $x_{0}, y_{0}, z_{0}$ (pointing out of the page). Frame $F_{1}$ rotated by the angle $\theta$ with respect to Frame $F_{0}$.

frame is considered, usually denoted by $i=0$, the superscript is omitted. Moreover, $r^{i}$ has components that lie along each of the coordinate axes of $F_{i}$.

Rotations between coordinate frames are represented by a rotation matrix $C_{b}^{a}$ belonging to the group $S O(3)$, which transforms a set of coordinates expressed in frame $F_{b}$ to coordinates expressed in frame $F_{a}[35]$. In particular, given frames $F_{0}$ and $F_{1}$, the columns of the rotation matrix $C_{1}^{0}$ consist of the direction cosines of the coordinates axes of $o_{1} x_{1} y_{1} z_{1}$ relative to the coordinate axes of $o_{0} x_{0} y_{0} z_{0}$ :

$$
C_{1}^{0}=\left[\begin{array}{ccc}
x_{1} \cdot x_{0} & y_{1} \cdot x_{0} & z_{1} \cdot x_{0} \\
x_{1} \cdot y_{0} & y_{1} \cdot y_{0} & z_{1} \cdot y_{0} \\
x_{1} \cdot z_{0} & y_{1} \cdot z_{0} & z_{1} \cdot z_{0}
\end{array}\right]
$$

Similarly, a vector $r^{1}$ expressed relative to the frame $F_{1}$ may be expressed relative to the frame $F_{0}$ by

$$
r^{0}=C_{1}^{0} r^{1}
$$

Rotation matrices of $S O(3)$ are orthonormal such that the inverse transformation is defined as $\left(C_{b}^{a}\right)^{-1}=C_{b}^{a T}=C_{a}^{b}$, and where $C_{b}^{a T} C_{b}^{a}=I$, where $I$ is an identity matrix. In this thesis rotation matrices between neighbouring frames, labelled $C_{i+1}^{i}$, will typically represent a rotation about one of the three Cartesian axes by some angle $\theta$. The three basic forms of the matrix are given below: 


$$
\begin{gathered}
y \text {-axis } \\
{\left[\begin{array}{ccc}
1 & 0 & 0 \\
0 & \cos (\theta) & -\sin (\theta) \\
0 & \sin (\theta) & \cos (\theta)
\end{array}\right]\left[\begin{array}{ccc}
\cos (\theta) & 0 & \sin (\theta) \\
0 & 1 & 0 \\
-\sin (\theta) & 0 & \cos (\theta)
\end{array}\right]\left[\begin{array}{ccc}
\cos (\theta) & -\sin (\theta) & 0 \\
\sin (\theta) & \cos (\theta) & 0 \\
0 & 0 & 1
\end{array}\right]}
\end{gathered}
$$

Also in this thesis, a rotation matrix of the form $C_{i}^{j}, j>i+1$, is equivalent to a cascade of rotations according to the current frame convention [35], where

$$
C_{i}^{j}=C_{j-1}^{j} C_{j-2}^{j-1} \cdots C_{i}^{i+1}
$$

Lastly, let $p \in \mathbb{R}^{3}$ be a vector and define the skew-symmetric operation

$$
p^{\times}:=\left[\begin{array}{ccc}
0 & -p_{3} & p_{2} \\
p_{3} & 0 & -p_{1} \\
-p_{2} & p_{1} & 0
\end{array}\right] .
$$

Thus the notation $(\cdot)^{\times}$produces a skew-symmetric matrix formed from a column vector. Note that the operator is linear, and that the inverse operation may be performed to extract the corresponding three-dimensional column vector.

\subsubsection{Three-Link Manipulator Model}

In this section we derive the model of a three-link manipulator used for control design and simulation. The approach makes use of the Euler-Lagrange equation and can be found in standard texts about robotics [35]. We begin by defining the Lagrangian

$$
\mathcal{L}=\mathcal{K}-\mathcal{P}
$$

where $\mathcal{K}$ is the total kinetic energy of the system, and $\mathcal{P}$ is the total potential energy of the system. These energies are expressed as functions of a set of generalized coordinates $q=$ $\left[\begin{array}{llll}q_{1} & q_{2} & \ldots & q_{n}\end{array}\right]^{T}$. For each coordinate $q_{i}$ the Euler-Lagrange equation [35] is given by

$$
\frac{d}{d t}\left(\frac{\partial \mathcal{L}}{\partial \dot{q}_{i}}\right)-\frac{\partial \mathcal{L}}{\partial q_{i}}=\tau_{i}, \quad i=1, \ldots, n
$$




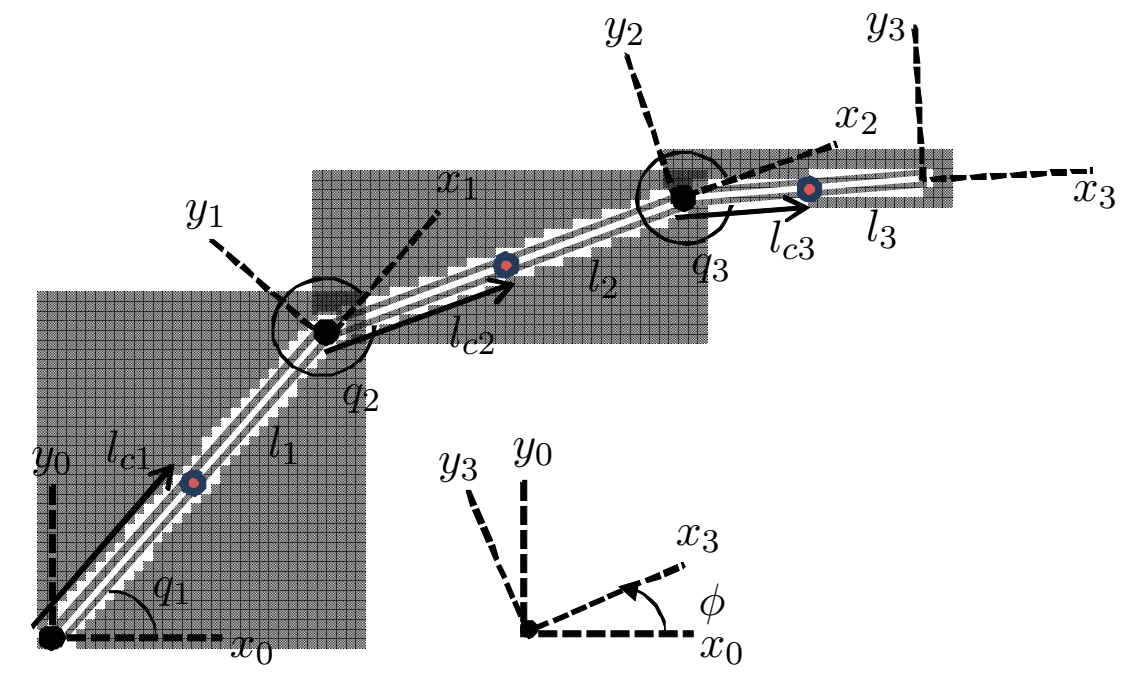

Figure 4.2: Notation for a Three-Link Planar Manipulator

where $\tau_{i}$ is an element of the a vector of generalized forces $\tau \in \mathbb{R}^{n}$. The immediate goal is to derive the expressions for $\mathcal{K}$ and $\mathcal{P}$ to generate (4.4) for each generalized coordinate. Consider the planar manipulator depicted in Figure 4.2 where $n=3$. The manipulator has three revolute joints represented by the generalized coordinate vector $q$ where $q=\left[\begin{array}{lll}q_{1} & q_{2} & q_{3}\end{array}\right]^{T}$. For the model derivation we use the standard Denavit-Hartenberg (DH) convention for the placement of the joint frames which consist of two assumptions [35]:

1. The axis $x_{i}$ is perpendicular to the axis $z_{i-1}$.

2. The axis $x_{i}$ intersects the axis $z_{i-1}$.

In the following let $F_{i}$, where $i \in \mathbb{R}$, denote a frame corresponding to coordinate axis $o_{i} x_{i} y_{i}$. Then in Figure 4.2 let the frame $F_{0}$ be an inertial frame fixed to joint 1 , at the base of link 1 . Frame $F_{1}$ is then rigidly attached to the end of link 1 to joint 2 , where axis $x_{1}$ is parallel to link 1 . Similarly, frame $F_{i}$ will be rigidly attached to the end of link $i$ to joint $i+1$, where axis $x_{i}$ is parallel to link $i$. This convention continues through all the links until the frame for the end effector is reached. Also, joints are labelled such that joint $i$ connects links $i-1$ and $i$. It can be verified by inspection that this frame placement satisfies the DH convention. 


\section{Dynamics}

Now for the dynamics derivation we consider the link lengths $l_{i}$, the link-centres $l_{c i}$, the masses $m_{l i}$ for each link, and moments of inertia $I_{i}$ of link $i$ about the axis directed out of the page through the centre of the link. This manipulator is assumed to operate in space where gravity effects are not considered, such that the potential energy term $\mathcal{P}$ is equal to zero. Therefore we consider the kinetic energy term that can be decomposed as $\mathcal{K}=\mathcal{K}_{T}+\mathcal{K}_{R}$, where $\mathcal{K}_{T}$ is the translational component, and $\mathcal{K}_{R}$ is the rotational component. To compute $\mathcal{K}_{T}$ we define the position vectors $r_{c i}$ that point from the base frame to the centre of each link

$$
\begin{aligned}
& r_{c 1}:=\left(l_{c 1} \cos q_{1}, l_{c 1} \sin q_{1}, 0\right) \\
& r_{c 2}:=\left(l_{1} \cos q_{1}+l_{c 2} \cos \left(q_{1}+q_{2}\right), l_{1} \sin q_{1}+l_{c 2} \sin \left(q_{1}+q_{2}\right), 0\right) \\
& r_{c 3}:=\left(l_{1} \cos q_{1}+l_{2} \cos \left(q_{1}+q_{2}\right)+l_{c 3} \cos \left(q_{1}+q_{2}+q_{3}\right), l_{1} \sin q_{1}+l_{2} \sin \left(q_{1}+q_{2}\right)+l_{c 3} \sin \left(q_{1}+q_{2}+q_{3}\right), 0\right) .
\end{aligned}
$$

One is able to obtain the linear velocity of the centre of mass of each link, defined $v_{c i}$, by taking the time derivative of the $r_{c i}, v_{c i}=\dot{r}_{c i}$. Then, $\mathcal{K}_{T}$ is the sum of the linear kinetic energies of each link. $\mathcal{K}_{T}$ can also be expressed in terms of the velocity vector of the generalized coordinates $\dot{q} \in \mathbb{R}^{3}$ using the relation $v_{c i}=\dot{r}_{c i}=J_{c i} \dot{q}$, where $J_{c i}$ represents the Jacobian matrix that maps the joint velocities $\dot{q}$ to the linear velocity vector $v_{c i}$. Therefore, we have

$$
\mathcal{K}_{T}=\frac{1}{2} m_{1} v_{c 1} v_{c 1}^{T}+\frac{1}{2} m_{2} v_{c 2} v_{c 2}^{T}+\frac{1}{2} m_{3} v_{c 3} v_{c 3}^{T}=\frac{1}{2} \dot{q}\left(m_{1} J_{c 1} J_{c 1}^{T}+m_{2} J_{c 2} J_{c 2}^{T}+m_{3} J_{c 3} J_{c 3}^{T}\right) \dot{q}
$$

For the manipulator above one can obtain the following expressions for the $J_{c i}$

$$
\begin{gathered}
J_{c 1}=\left[\begin{array}{ccc}
-l_{c 1} \sin q_{1} & 0 & 0 \\
l_{c 1} \cos q_{1} & 0 & 0 \\
0 & 0 & 0
\end{array}\right], \quad J_{c 2}=\left[\begin{array}{ccc}
-l_{1} \sin q_{1}-l_{c 2} \sin q_{1}+q_{2} & -l_{c 2} \sin q_{1}+q_{2} & 0 \\
l_{1} \cos q_{1}+l_{c 2} \cos q_{1}+q_{2} & +l_{c 2} \cos q_{1}+q_{2} & 0 \\
0 & 0 & 0
\end{array}\right], \\
J_{c 3}=\left[\begin{array}{ccc}
-l_{1} \sin q_{1}-l_{2} \sin \left(q_{1}+q_{2}\right)-l_{c 3} \sin \left(q_{1}+q_{2}+q_{3}\right) & -l_{2} \sin \left(q_{1}+q_{2}\right)-l_{c 3} \sin \left(q_{1}+q_{2}+q_{3}\right) & -l_{c 3} \sin \left(q_{1}+q_{2}+q_{3}\right) \\
l_{1} \cos q_{1}+l_{2} \cos \left(q_{1}+q_{2}\right)-l_{c 3} \cos \left(q_{1}+q_{2}+q_{3}\right) & l_{2} \cos \left(q_{1}+q_{2}\right)-l_{c 3} \cos \left(q_{1}+q_{2}+q_{3}\right) & l_{c 3} \cos \left(q_{1}+q_{2}+q_{3}\right) \\
0 & 0 & 0
\end{array}\right] .
\end{gathered}
$$

For rotational energy $\mathcal{K}_{R}$ we first express the angular velocity of each link, $\omega_{i}$, with respect to the base inertial frame, in terms of the joint rates. Let $\hat{k}:=(0,0,1)$ be the unit vector pointing 
out of the page so that we have

$$
\omega_{1}=\dot{q}_{1} \hat{k}, \quad \omega_{2}=\left(\dot{q}_{1}+\dot{q}_{2}\right) \hat{k}, \quad \omega_{3}=\left(\dot{q}_{1}+\dot{q}_{2}+\dot{q}_{3}\right) \hat{k}
$$

Notice how the angular velocity of link $i$ depends on the velocity of link $j$ where $j<i$. By summing terms of the form $\omega_{i}^{T} I_{i} \omega_{i}[35]$, we obtain

$$
\mathcal{K}_{R}=\frac{1}{2} \dot{q}\left(I_{1}\left[\begin{array}{ccc}
1 & 0 & 0 \\
0 & 0 & 0 \\
0 & 0 & 0
\end{array}\right]+I_{2}\left[\begin{array}{lll}
1 & 1 & 0 \\
1 & 1 & 0 \\
0 & 0 & 0
\end{array}\right]+I_{3}\left[\begin{array}{ccc}
1 & 1 & 1 \\
1 & 1 & 1 \\
1 & 1 & 1
\end{array}\right]\right) \dot{q}
$$

The addition of the translational and rotational energy terms yields the total kinetic energy

$$
\mathcal{K}=\mathcal{K}_{T}+\mathcal{K}_{R}=\frac{1}{2} \dot{q} M(q) \dot{q},
$$

where $M(q)$ is an $n \times n$ symmetric, positive definite matrix called the inertia matrix. After the necessary algebraic steps and applying relevant trigonometric identities the $i j$ entries of the $M$ matrix for this robotic manipulator are [35]:

$$
\begin{aligned}
& M_{11}=\left(I_{1}+I_{2}+I_{3}+l_{1}^{2} m_{l 2}+l_{1}^{2} m_{l 3}+l_{2}^{2} m_{l 3}+l_{c 1}^{2} m_{l 1}+l_{c 2}^{2} m_{l 2}+l_{c 3}^{2} m_{l 3}+2 l_{1} l_{c 3} m_{l 3} \cos \left(q_{2}+q_{3}\right)+\right. \\
& \left.2 l_{1} l_{2} m_{l 3} \cos \left(q_{2}\right)+2 l_{1} l_{c 2} m_{l 2} \cos \left(q_{2}\right)+2 l_{2} l_{c 3} m_{l 3} \cos \left(q_{3}\right)\right) \\
& M_{21}=\left(m_{l 3} l_{2}^{2}+2 m_{l 3} \cos \left(q_{3}\right) l_{2} l_{c 3}+l_{1} m_{l 3} \cos \left(q_{2}\right) l_{2}+m_{l 2} l_{c 2}^{2}+l_{1} m_{l 2} \cos \left(q_{2}\right) l_{c 2}+m_{l 3} l_{c 3}^{2}+l_{1} m_{l 3} \cos \left(q_{2}+\right.\right. \\
& \left.\left.q_{3}\right) l_{c 3}+I_{2}+I_{3}\right) \\
& M_{31}=\left(m_{l 3} l_{c 3}^{2}+l_{2} m_{l 3} \cos \left(q_{3}\right) l_{c 3}+I_{3}\right) \\
& M_{22}=\left(m_{l 3} l_{2}^{2}+2 m_{l 3} \cos \left(q_{3}\right) l_{2} l_{c 3}+m_{l 2} l_{c 2}^{2}+m_{l 3} l_{c 3}^{2}+I_{2}+I_{3}\right) \\
& M_{32}=\left(m_{l 3} l_{c 3}^{2}+l_{2} m_{l 3} \cos \left(q_{3}\right) l_{c 3}+I_{3}\right) \\
& M_{33}=\left(m_{l 3} l_{c 3}^{2}+I_{3}\right) \\
& M_{12}=M_{21}, \quad M_{13}=M_{31}, \quad M_{23}=M_{32} .
\end{aligned}
$$

With $\mathcal{K}$ defined, the application of Equation (4.4) for each $q_{i}$ collectively yields a set of terms that multiply with the acceleration vector $\ddot{q}$, and a second set of terms that multiply with the rate vector $\dot{q}$. These second terms are associated with centrifugal and Coriolis dynamics, which are compactly organized into a matrix $C(q, \dot{q}) \in \mathbb{R}^{n \times n}[35]$. The elements of $C(q, \dot{q})$ are called 
Chirsotffel Symbols of the First Kind [35], and are derived from the entries of $M(q)$ by

$$
c_{k j}:=\sum_{i=1}^{n} \frac{1}{2}\left(\frac{\partial M_{k j}}{\partial q_{i}}+\frac{\partial M_{k i}}{\partial q_{j}}-\frac{\partial M_{i j}}{\partial q_{k}}\right) \dot{q}_{i} .
$$

The $k j$ entries of the $C$ matrix for this robotic manipulator are:

$$
\begin{aligned}
& C_{11}=-\left(\dot{q}_{2}+\dot{q}_{3}\right) l_{1} l_{c 3} m_{l 3} \sin \left(q_{2}+q_{3}\right)-\dot{q}_{2} l_{1} l_{2} m_{l 3} \sin \left(q_{2}\right)-\dot{q}_{2} l_{1} l_{c 2} m_{l 2} \sin \left(q_{2}\right)-\dot{q}_{3} l_{2} l_{c 3} m_{l 3} \sin \left(q_{3}\right) \\
& C_{21}=\dot{q}_{1} l_{1} l_{c 3} m_{l 3} \sin \left(q_{2}+q_{3}\right)+\dot{q}_{1} l_{1} l_{2} m_{l 3} \sin \left(q_{2}\right)+\dot{q}_{1} l_{1} l_{c 2} m_{l 2} \sin \left(q_{2}\right)-\dot{q}_{3} l_{2} l_{c 3} m_{l 3} \sin \left(q_{3}\right) \\
& C_{31}=l_{c 3} m_{l 3}\left(\dot{q}_{1} l_{2} \sin \left(q_{3}\right)+\dot{q}_{2} l_{2} \sin \left(q_{3}\right)+\dot{q}_{1} l_{1} \sin \left(q_{2}+q_{3}\right)\right) \\
& C_{12}=-\left(\dot{q}_{1}+\dot{q}_{2}+\dot{q}_{3}\right) l_{1} l_{c 3} m_{l 3} \sin \left(q_{2}+q_{3}\right)-\left(\dot{q}_{1}+\dot{q}_{2}\right) l_{1} l_{2} m_{l 3} \sin \left(q_{2}\right)-\left(\dot{q}_{1}+\dot{q}_{2}\right) l_{1} l_{c 2} m_{l 2} \sin \left(q_{2}\right)- \\
& \dot{q}_{3} l_{2} l_{c 3} m_{l 3} \sin \left(q_{3}\right) \\
& C_{22}=-\dot{q}_{3} l_{2} l_{c 3} m_{l 3} \sin \left(q_{3}\right) \\
& C_{32}=l_{2} l_{c 3} m_{l 3} \sin \left(q_{3}\right)\left(\dot{q}_{1}+\dot{q}_{2}\right) \\
& C_{13}=-l_{c 3} m_{l 3}\left(l_{1} \sin \left(q_{2}+q_{3}\right)+l_{2} \sin \left(q_{3}\right)\right)\left(\dot{q}_{1}+\dot{q}_{2}+\dot{q}_{3}\right) \\
& C_{23}=-l_{2} l_{c 3} m_{l 3} \sin \left(q_{3}\right)\left(\dot{q}_{1}+\dot{q}_{2}+\dot{q}_{3}\right) \\
& C_{33}=0 .
\end{aligned}
$$

The collection of $M(q), C(q, \dot{q})$, and generalized torque $\tau$ yield the dynamic equation of the planar three-link manipulator in (4.7) below. The torque $\tau \in \mathbb{R}^{n}$ is the vector of control inputs at the joints. The additional term $J(q)^{T} F_{\text {ext }}$ is the standard representation for the effects of an external force $F_{\text {ext }} \in \mathbb{R}^{6}$ consisting of three orthogonal force and moment components [35]; the effects of this force are mapped to a torque acting on each generalized coordinate by the transpose of the system Jacobian $J(q) \in \mathbb{R}^{6 \times n}$ :

$$
M(q) \ddot{q}+C(q, \dot{q}) \dot{q}=\tau+J(q)^{T} F_{\text {ext }} .
$$

\section{Forward and Velocity Kinematics}

While the expressions above yield the system dynamics, we must also compute the forward kinematics and the velocity kinematics for the control design process. The forward kinematics provide expressions for the end effector Cartesian position in terms of the joint variables, as well as the orientation parametrized by three angles; this set of six quantities is called the task space coordinates [35]. However, the manipulator of Figure 4.2 is planar in the $x_{0}-y_{0}$ plane, 
such that the only task space coordinates of interest are the end effector position coordinates $\left(x_{e}, y_{e}\right)$, and the end effector angle denoted by $\phi$, which represents the angle between coordinate axes $x_{0}$ and $x_{3}$; i.e. when $\phi=0$ then $x_{0}$ and $x_{3}$ are parallel. The expressions for these quantities may be derived by inspection of Figure 4.2 and are collected below:

$$
\begin{aligned}
x_{e} & =l_{1} \cos \left(q_{1}\right)+l_{2} \cos \left(q_{1}+q_{2}\right)+l_{3} \cos \left(q_{1}+q_{2}+q_{3}\right) \\
y_{e} & =l_{1} \sin \left(q_{1}\right)+l_{2} \sin \left(q_{1}+q_{2}\right)+l_{3} \sin \left(q_{1}+q_{2}+q_{3}\right) \\
{\left[\begin{array}{cc}
x_{3} \cdot x_{0} & y_{3} \cdot x_{0} \\
x_{3} \cdot y_{0} & y_{3} \cdot y_{0}
\end{array}\right] } & =\left[\begin{array}{cc}
\cos \left(q_{1}+q_{2}+q_{3}\right) & -\sin \left(q_{1}+q_{2}+q_{3}\right) \\
\sin \left(q_{1}+q_{2}+q_{3}\right) & \cos \left(q_{1}+q_{2}+q_{3}\right)
\end{array}\right] \\
\Longrightarrow \phi & =q_{1}+q_{2}+q_{3} .
\end{aligned}
$$

The matrix above is called the orientation matrix and is constructed by the direction cosines of the $x_{3}$ and $y_{3}$ axes relative to the base axes $x_{0}$ and $y_{0}$. The simple form of the matrix allows us to completely characterize its entries by $\phi$.

Next we derive the Jacobian used for the velocity kinematics. The Jacobian is a $6 \times n$ matrix that relates joint velocities to the six possible task space motions of the end effector. As such it is constructed with terms that account for both linear and angular velocities of the end effector with respect to each joint. If Joint $i$ is revolute, then the $i$-th column $J_{i}$ of the Jacobian is defined as [35]

$$
J_{i}=\left[\begin{array}{c}
z_{i-1} \times\left(o_{n}-o_{i-1}\right) \\
z_{i-1}
\end{array}\right], \quad i \in\{1,2,3\}, n=3,
$$

where $z_{i-1}=(0,0,1)$ is an axis of rotation for this manipulator, $o_{n}$ is the position vector of the origin of frame $F_{n}$ with respect to the base, and similarly $o_{i-1}$ is position vector of frame $F_{i-1}$. The upper row block is the linear Jacobian, and the lower row block is the angular Jacobian. Since the control objective requires the regulation of only three variables $\left(x_{e}, y_{e}, \phi\right)$, the Jacobian can be reduced to a $3 \times 3$ matrix (the rows dropped are those for task space coordinates that cannot be modulated by the degrees of freedom of this manipulator and so are 
zero vectors). We obtain

$$
\left[\begin{array}{c}
\dot{x}_{e} \\
\dot{y}_{e} \\
\dot{\phi}
\end{array}\right]=J\left[\begin{array}{c}
\dot{q}_{1} \\
\dot{q}_{2} \\
\dot{q}_{3}
\end{array}\right]
$$

where

$$
J=\left[\begin{array}{ccc}
-\left(l_{1} \sin \left(q_{1}\right)+b+a\right) & -(b+a) & -a \\
l_{1} \cos \left(q_{1}\right)+d+c & d+c & c \\
1 & 1 & 1
\end{array}\right]
$$

with

$$
\begin{array}{cc}
a=l_{3} \sin \left(q_{1}+q_{2}+q_{3}\right) & b=l_{2} \sin \left(q_{1}+q_{2}\right) \\
c=l_{3} \cos \left(q_{1}+q_{2}+q_{3}\right) & d=l_{2} \cos \left(q_{1}+q_{2}\right) .
\end{array}
$$

The Jacobian is invertible except in the singular configurations of the manipulator. We have $\operatorname{det} J=l_{1} l_{2} \sin \left(q_{2}\right)$ so the only singularities occur when $q_{2}=0$ or $180^{\circ}$.

\section{Feedback Linearisation}

As can be discerned by the dynamics of (4.7), manipulator systems are highly non-linear, and require unique approaches to obtain reliable control. In this development we will simplify the control of the system by performing two feedback linearisations in order to transform the nonlinear model into a linear one. Such an approach is common in the literature $[7,8,35]$. The

first feedback transformation allows direct control of the joints in joint space. Let $U_{q} \in \mathbb{R}^{3}$ be a new exogenous input and define the feedback transformation

$$
\tau=M(q) U_{q}+C(q, \dot{q}) \dot{q}-J(q)^{T} F_{\text {ext }} .
$$

Substituting this equation into the dynamics (4.7) and cancelling terms we obtain the linearised dynamics

$$
\ddot{q}=U_{q}
$$

Now each element in $U_{q}$ can be independently assigned to control each joint independently. Of course, in practice perfect cancellation is not possible and is also dependent on the computa- 
tional power and accuracy of the hardware. Next we perform a second feedback transformation in order to work in task space. First we take the time derivative of (4.10), and by the chain rule we get

$$
\frac{\partial}{\partial t}\left[\begin{array}{c}
\dot{x}_{e} \\
\dot{y}_{e} \\
\dot{\phi}
\end{array}\right]=J\left[\begin{array}{c}
\ddot{q}_{1} \\
\ddot{q}_{2} \\
\ddot{q}_{3}
\end{array}\right]+\dot{J}\left[\begin{array}{c}
\dot{q}_{1} \\
\dot{q}_{2} \\
\dot{q}_{3}
\end{array}\right] .
$$

Now let $U=\left(u_{x_{e}}, u_{y_{e}}, u_{\phi}\right) \in \mathbb{R}^{3}$ be a second exogenous input and define a second feedback transformation

$$
U_{q}=J^{-1}\left(\left[\begin{array}{c}
u_{x_{e}} \\
u_{y_{e}} \\
u_{\phi}
\end{array}\right]-\dot{J}\left[\begin{array}{c}
\dot{q}_{3} \\
\dot{q}_{4} \\
\dot{q}_{5}
\end{array}\right]\right)
$$

Combining (4.14) and (4.13) with (4.12) and cancelling terms we get

$$
\left[\begin{array}{c}
\ddot{x}_{e} \\
\ddot{y}_{e} \\
\ddot{\phi}_{e}
\end{array}\right]=\left[\begin{array}{l}
u_{x_{e}} \\
u_{y_{e}} \\
u_{\phi_{e}}
\end{array}\right]
$$

Equation (4.15) yields three uncoupled double integrator systems so that each element of $U$ can be independently assigned to independently control each task space variable. Note that to construct the feedback transformation (4.15) the inverse $J^{-1}$ and the element-wise time derivative $\dot{J}$ must be calculated. The need to avoid singularities is evident otherwise $J^{-1}$ would not be defined.

We briefly comment on the differences between synthesizing a control law in joint space to one in task space (which is the approach adopted here). Figures $4.3-4.4$ found in [36] reflect the two approaches for the step response of an underdamped mechancial system. The graphs on the left, denoted 'Joint Response', depict a ' $q_{2}$ vs. $q_{1}$ ' trajectory, while the graphs on the right, denoted 'Position Response', depict a ' $y$ vs. $x$ ' trajectory. Figure 4.3 depicts control in joint space, where a straight-line trajectory of the joint variables may be readily specified and result in a curved trajectory in task space. On the contrary, Figure 4.4 depicts control in task space, which can more readily yield a straight-line trajectory of the task space coordinates and 


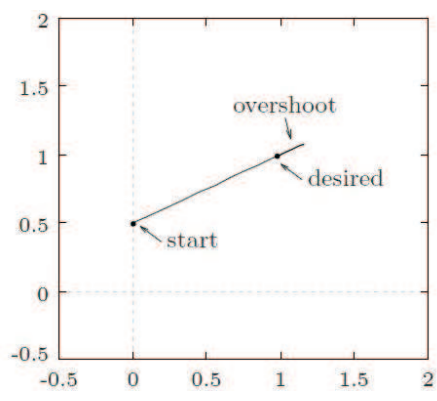

(a)

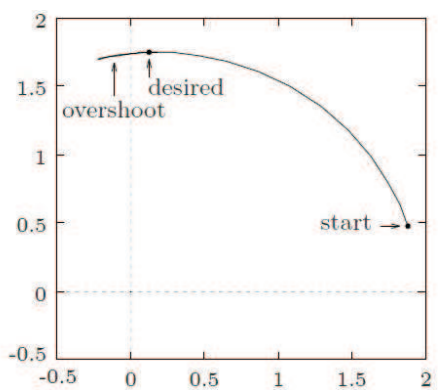

(b)

Figure 4.3: Control in Joint Space: (a) Joint Response, (b) Position Response

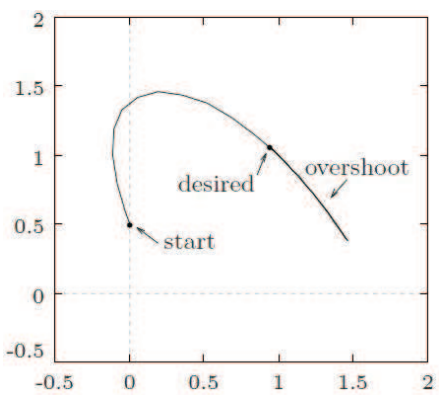

(a)

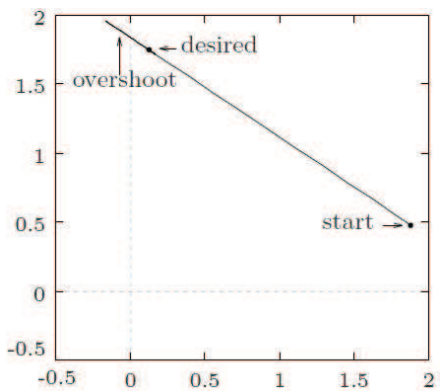

(b)

Figure 4.4: Control in Task Space: (a) Joint Response, (b) Position Response

produce a curved trajectory in joint space. In this thesis it was chosen to frame the context of the problem in terms of the end effector task space variables. As will be shown, this requires the definition of constraints on a subset of the task space variables in the form of linear inequalities. The problem definition therefore lends itself to the use of task space control. Ultimately, this decision represents a trade-off in regards to computational complexity, as the additional layer of control in (4.14) is required, as well as the need to avoid singularities where $J^{-1}$ is undefined.

Thus we have completed the background material for the three-link manipulator. In the section to follow we will use the dynamical model (4.7), the forward and velocity kinematic equations of (4.8) and (4.11), and the general procedure of feedback linearisation to control the task space variables. 


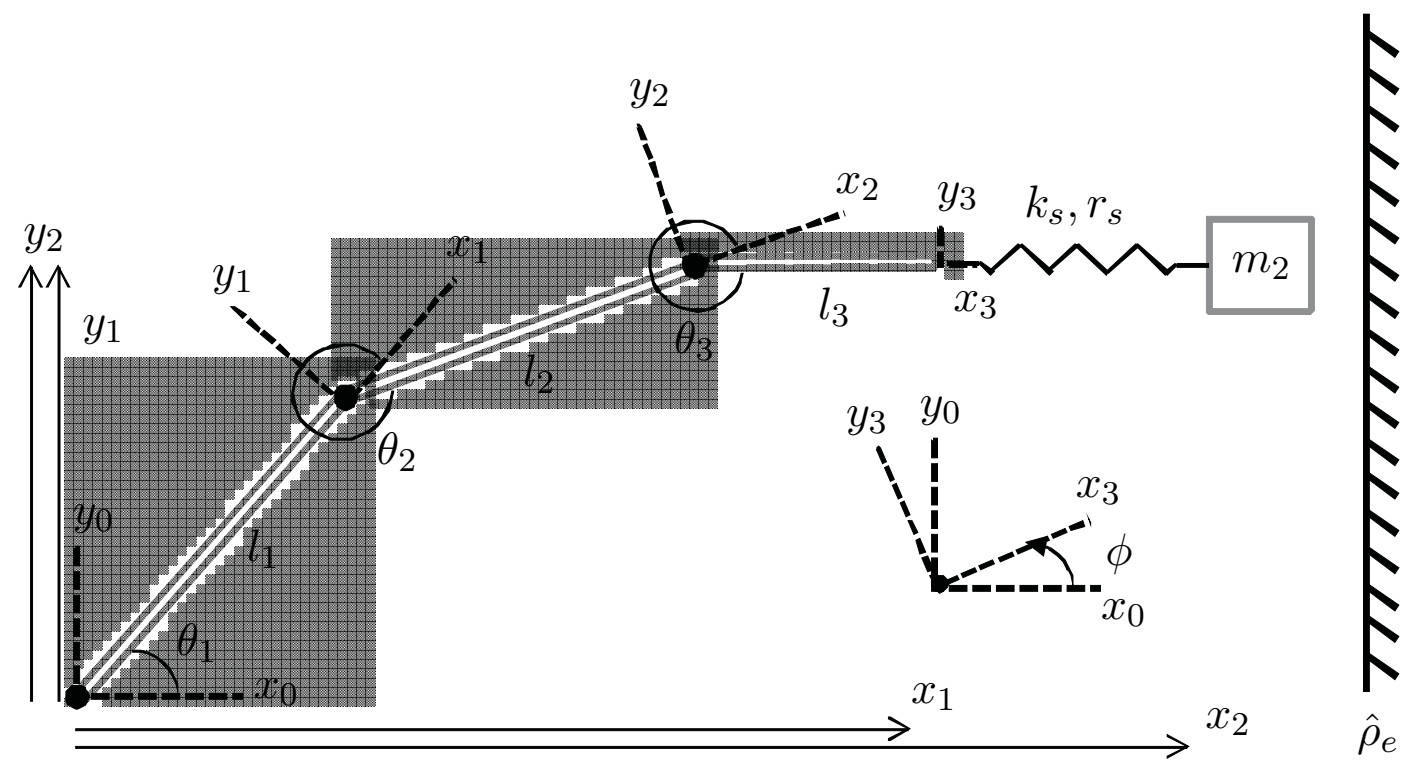

Figure 4.5: Three-link Manipulator with second mass $m_{2}$.

\subsection{System Modelling with Three-Link Manipulator}

In this section we present the manipulator system under consideration, and derive simplified state space models with which a solution to the bouncing problem is derived. Consider the system illustrated in Figure 4.5 consisting of a three link manipulator whose base is fixed at frame $F_{0}$, and has end effector frame $F_{3}$. Attached to the end effector is a second mass $m_{2}$, connected through a spring such that the length of the spring is parallel to the $x_{3}$-axis. Let $\left(x_{1}, y_{1}\right)$ and $\left(x_{2}, y_{2}\right)$ be the position of the end effector and second mass respectively, with respect to the base frame. Moreover let $k_{s}$ and $r_{s}$ represent the stiffness and rest-length of the connecting spring, and denote $F_{e}$ to be the external force acting on the second mass when it is in contact with an obstacle at the location $\left(\hat{\rho}_{e}, 0\right)$.

The robotic manipulator dynamics are described by the dynamical equation of the previous section where $n=3$

$$
M(\theta) \ddot{\theta}+C(\theta, \dot{\theta}) \dot{\theta}+N(\theta, \dot{\theta})=\tau+J(\theta)^{T} F_{\text {ext }} .
$$

The vector $\theta \in \mathbb{R}^{3}$ is the vector of joint angles, $M(\theta) \in \mathbb{R}^{3 \times 3}$ is the inertia matrix of the manipulator, $C(\theta, \dot{\theta}) \in \mathbb{R}^{3 \times 3}$ models the Coriolis and centrifugal forces, $N(\theta, \dot{\theta}) \in \mathbb{R}^{n}$ models potential energy terms, i.e. gravity effects, $\tau \in \mathbb{R}^{3}$ is a vector of torque inputs applied at the 
joints, $J(\theta) \in \mathbb{R}^{3 \times 3}$ is the Jacobian, and $F_{\text {ext }} \in \mathbb{R}^{3}$ is a vector defining external forces acting on the end effector, all expressed with respect to the base frame. For the system of Figure 4.5 the external force acting on the manipulator is taken to be the force that acts between the manipulator and the second mass such that $F_{\text {ext }}=F_{s}$ where $F_{s} \in \mathbb{R}^{3}$ is called the sensed force. For this problem three assumptions are made that simplify the dynamics of the manipulator.

\section{Assumption 4.1.}

(B1) Actuator dynamics are ignored, rather input torques are applied directly to the joints.

(B2) The manipulator operates in space, thus $N(\theta, \dot{\theta})=0$.

(B3) The slow motion of the manipulator renders negligible the effects of the centrifugal and Coriolis term, thus $C(\theta, \dot{\theta}) \dot{\theta}=0$.

Using the above assumptions we obtain the reduced rate-linear approximation

$$
M(\theta) \ddot{\theta}=\tau+J(\theta)^{T} F_{s} .
$$

To aid in the control of the manipulator we compute the forward kinematics and the Jacobian for the velocity kinematics. By inspection it is clear that the frames in Figure 4.5 conform with the Denavit-Hartenberg (DH). As in the previous section, the forward kinematics provide expressions for the task space coordinates; namely, the end effector position, denoted $\left(x_{1}, y_{1}\right)$, with respect to the base, as well as the orientation parametrized by the angle $\phi$ :

$$
\begin{aligned}
x_{1} & =l_{1} \cos \left(\theta_{1}\right)+l_{2} \cos \left(\theta_{1}+\theta_{2}\right)+l_{3} \cos \left(\theta_{1}+\theta_{2}+\theta_{3}\right) \\
y_{1} & =l_{1} \sin \left(\theta_{1}\right)+l_{2} \sin \left(\theta_{1}+\theta_{2}\right)+l_{3} \sin \left(\theta_{1}+\theta_{2}+\theta_{3}\right) \\
{\left[\begin{array}{cc}
x_{3} \cdot x_{0} & y_{3} \cdot x_{0} \\
x_{3} \cdot y_{0} & y_{3} \cdot y_{0}
\end{array}\right] } & =\left[\begin{array}{cc}
\cos \left(\theta_{1}+\theta_{2}+\theta_{3}\right) & -\sin \left(\theta_{1}+\theta_{2}+\theta_{3}\right) \\
\sin \left(\theta_{1}+\theta_{2}+\theta_{3}\right) & \cos \left(\theta_{1}+\theta_{2}+\theta_{3}\right)
\end{array}\right] \\
\Longrightarrow \phi & =\theta_{1}+\theta_{2}+\theta_{3} .
\end{aligned}
$$

Next we derive the Jacobian used for the velocity kinematics. Since the control objective requires the regulation of only three variables $\left(x_{1}, y_{1}, \phi\right)$, the Jacobian can be reduced to a $3 \times 3$ 
matrix. Application of (4.9) using (4.18) produces

$$
\left[\begin{array}{c}
\dot{x}_{1} \\
\dot{y}_{1} \\
\dot{\phi}
\end{array}\right]=J\left[\begin{array}{c}
\dot{\theta}_{1} \\
\dot{\theta}_{2} \\
\dot{\theta}_{3}
\end{array}\right]
$$

where

$$
J=\left[\begin{array}{ccc}
-\left(l_{1} \sin \left(\theta_{1}\right)+b+a\right) & -(b+a) & -a \\
l_{1} \cos \left(\theta_{1}\right)+d+c & d+c & c \\
1 & 1 & 1
\end{array}\right]
$$

with

$a=l_{3} \sin \left(\theta_{1}+\theta_{2}+\theta_{3}\right) \quad b=l_{2} \sin \left(\theta_{1}+\theta_{2}\right) \quad c=l_{3} \cos \left(\theta_{1}+\theta_{2}+\theta_{3}\right) \quad d=l_{2} \cos \left(\theta_{1}+\theta_{2}\right)$.

We proceed now to transform the non-linear dynamics of (4.17) into a linear form with respect to the task space coordinates. As in the previous section we perform an initial feedback transformation to directly control the three joint dynamics. Let $U_{\theta} \in \mathbb{R}^{3}$ be a new exogenous input and define the feedback transformation $\tau=M(\theta) U_{\theta}-J(\theta)^{T} F_{s}$. Substituting this expression into (4.17) and cancelling terms we obtain

$$
\ddot{\theta}=U_{\theta}
$$

Let $m_{1}>0$ be a to-be-determined parameter for the first mass, $U \in \mathbb{R}^{3}$ a second exogenous input, and define a second feedback transformation

$$
U_{\theta}=J^{-1}(\theta)\left(\frac{1}{m_{1}} U+\frac{1}{m_{1}} F_{s}-\dot{J}(\theta)\left[\begin{array}{c}
\dot{\theta}_{1} \\
\dot{\theta}_{2} \\
\dot{\theta}_{3}
\end{array}\right]\right)
$$




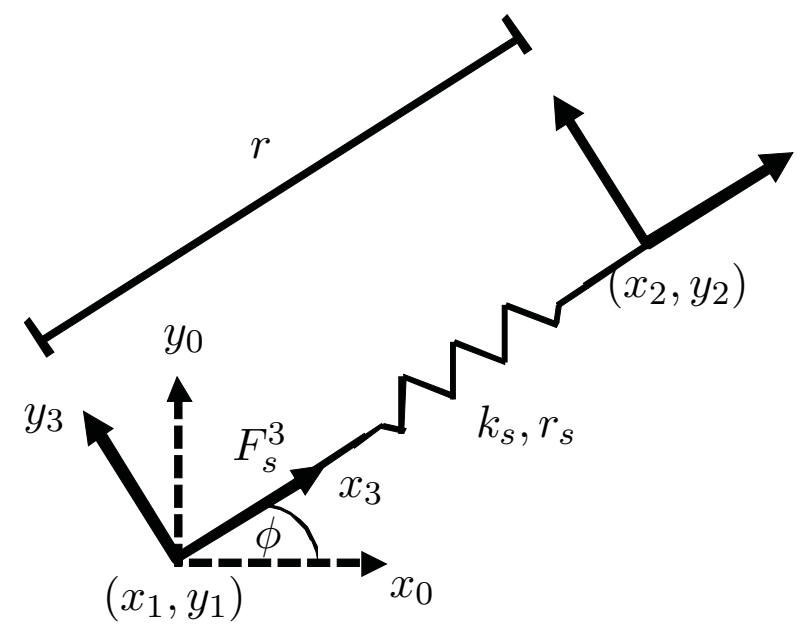

Figure 4.6: End effector Frame and the Spring-Force connecting the Second Mass.

Using the development of (4.13)-(4.15), the transformation (4.22) generates the dynamics

$$
m_{1}\left[\begin{array}{c}
\ddot{x}_{1} \\
\ddot{y}_{1} \\
\ddot{\phi}_{1}
\end{array}\right]=U+F_{s} .
$$

Equation (4.23) yields three uncoupled double integrator systems so that each element of $U$ can be independently assigned to independently control each task space variable.

Next we seek an expression for the spring force $F_{s}$ that acts between the manipulator end effector and second mass. Once this expression is obtained the dynamics of the second mass may be derived. Due to the planar configuration of the manipulator, $F_{s}$ has two translational components along the $x_{0}$ and $y_{0}$ axes, denoted $F_{s_{x}}$ and $F_{s_{y}}$, and one rotational component about the $z$-axis, denoted $F_{s_{\phi}}$. To start, we consider the sensed force expressed in the end effector frame $F_{3}$, denoted $F_{s}^{3}$. Figure 4.6 depicts frame $F_{3}$ and the spring connection to the second mass. The force $F_{s}^{3}$ depends on the displacement between the end effector and second mass, denoted $r \in \mathbb{R}^{2}$, whose magnitude is given by

$$
\|r\|=\left\|\left(x_{2}, y_{2}\right)-\left(x_{1}, y_{1}\right)\right\|
$$

By construction the motion of the second mass is constrained along the $x_{3}$-axis. Consequently, the orientation of the second mass will be identical to that of the end effector as parametrized 
by $\phi$. Moreover, only the $x$-component of $F_{s}^{3}$ is non-zero such that

$$
F_{s}^{3}=\left(k_{s}\left(\|r\|-r_{s}\right), 0,0\right) .
$$

Then through trigonometry the magnitude of $r$ is related to the coordinates $x_{2}$ and $x_{1}$ (assuming $\left.x_{2}>x_{1}\right)$ by

$$
\|r\|=\frac{x_{2}-x_{1}}{\cos (\phi)}
$$

Note, the above expression is only admissible for $|\phi|<90^{\circ}$. Substitution of (4.26) into (4.25) yields

$$
F_{s}^{3}=\left(k_{s}\left(\frac{x_{2}-x_{1}}{\cos (\phi)}-r_{s}\right), 0,0\right)
$$

The expression for $F_{s}$ in the base frame is obtained by rotating $F_{s}^{3}$ by means of a rotation matrix $R_{3}^{0}$

$$
R_{3}^{0}=\left[\begin{array}{ccc}
\cos (\phi) & -\sin (\phi) & 0 \\
\sin (\phi) & \cos (\phi) & 0 \\
0 & 0 & 1
\end{array}\right]
$$

such that

$$
F_{s}=\left[\begin{array}{c}
F_{s_{x}} \\
F_{s_{y}} \\
F_{s_{\phi}}
\end{array}\right]=R_{3}^{0} F_{s}^{3}=\left[\begin{array}{c}
k_{s}\left(x_{2}-x_{1}-\cos (\phi) r_{s}\right) \\
k_{s}\left(\left(x_{2}-x_{1}\right) \tan (\phi)-\sin (\phi) r_{s}\right) \\
0
\end{array}\right]
$$

Note that $F_{s}$ is used in the feedback linearisation of the manipulator (4.22), and dynamics of the task space variables (4.23). From the expression for $F_{s}$ it is clear that we need only model the dynamics associated with position coordinate $x_{2}$ of the second mass with respect to the base. Then, the coordinate $y_{2}$ can be directly computed by

$$
y_{2}=y_{1}+\left(x_{2}-x_{1}\right) \tan (\phi) .
$$

Consider then the states of the second mass $\left(x_{2}, \dot{x}_{2}\right)$ representing the displacement and velocity along the $x_{0}$ axis. To derive the necessary equation of motion we start with the 
expression for the linear momentum of the second mass [5]

$$
p_{2}=m_{2} v_{2}-r_{2}^{\times} \omega_{2}
$$

where $p_{2} \in \mathbb{R}^{3}$ is the linear momentum of the second mass, $m_{2} \in \mathbb{R}$ is the mass, $v_{2} \in \mathbb{R}^{3}$ is the velocity of the center of mass, $r_{2} \in \mathbb{R}^{3}$ is the position of the center of mass, and $\omega_{2} \in \mathbb{R}^{3}$ is the angular velocity, which are all quantified with respect to the base frame. In particular, the angular velocity of the second mass will be the same as that of the manipulator end effector, so $r_{2}$ and $\omega_{2}$ can be defined as

$$
r_{2}:=\left(x_{2}, y_{2}, 0\right) \quad \omega_{2}:=(0,0, \dot{\phi})
$$

The desired equation of motion is obtained by differentiation with respect to time of the linear momentum, which is then equated to the applied forces. Assuming again slow motion of the manipulator system, we drop the non-linear centrifugal and Coriolis terms appearing from the differentiation [5], producing the linear expression

$$
m_{2} \dot{v}_{2}-r_{2}^{\times} \dot{\omega}_{2}=-\left[\begin{array}{c}
F_{s_{x}} \\
F_{s_{y}} \\
0
\end{array}\right]+F_{e},
$$

which may also be expressed as

$$
m_{2}\left[\begin{array}{c}
\ddot{x}_{2} \\
\ddot{y}_{2} \\
\ddot{z}_{2}
\end{array}\right]=r_{2}^{\times}\left[\begin{array}{l}
0 \\
0 \\
\ddot{\phi}
\end{array}\right]-\left[\begin{array}{c}
F_{s_{x}} \\
F_{s_{y}} \\
0
\end{array}\right]+F_{e} .
$$

The dynamic equation for $x_{2}$ may be extracted from the top row of (4.32), which is forthcoming.

Therefore we have derived a two-mass model shown in Figure 4.7 consisting of (4.23) and (4.32). This two-mass system is a general result based on the architecture of the serial manipulator and the use of feedback linearisation. However, the design herein marks the beginning of the contributions of this thesis where the two-mass model is simplified to a single-mass system 


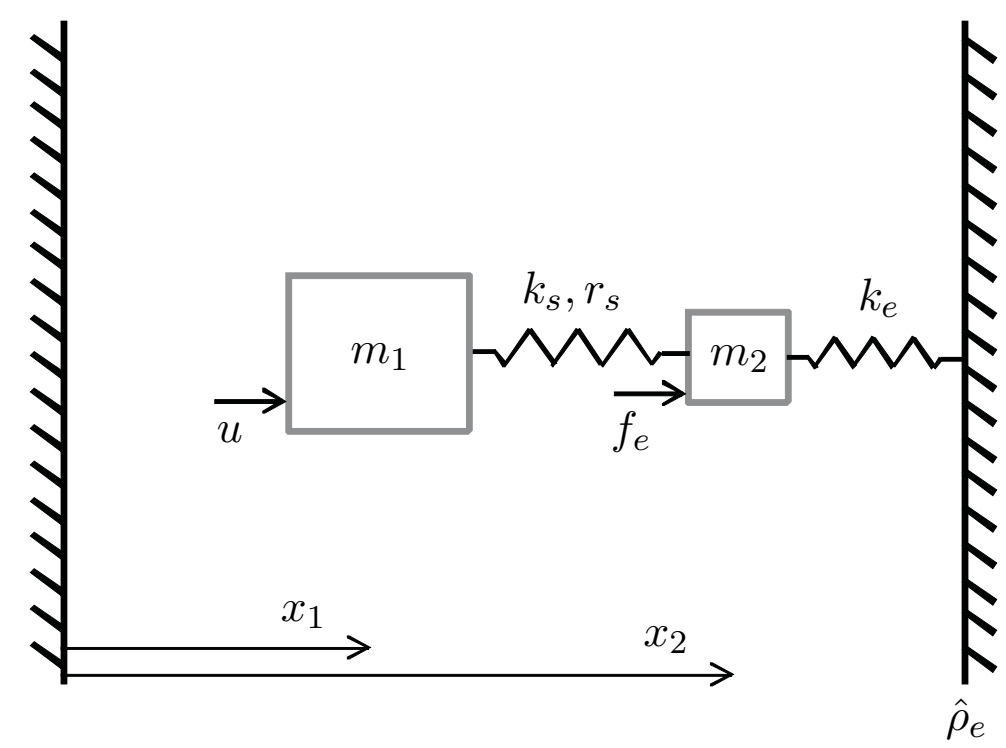

Figure 4.7: Two-mass model consisting of the feedback-linearised manipulator, $m_{1}$, and second mass $m_{2}$.

in order to obtain state space models suitable for control design. The derivation herein relies on three assumptions summarised as follows.

\section{Assumption 4.2.}

(C1) A one degree-of-freedom motion occurs along the $x_{0}$-axis to contact the environment in the normal direction. Therefore, $\phi=\theta_{1}+\theta_{2}+\theta_{3}=0^{\circ}$.

(C2) $m_{1}>>m_{2}$

(C3) During contact $\ddot{x}_{2} \simeq 0$.

The above assumptions enter into the design as follows. First, assumption (C1) means that the second mass and manipulator end effector only move horizontally; such behaviour is feasible as the manipulator's three joints allow for orientation control. Under this assumption let $f_{s} \in \mathbb{R}$ be the first component of $F_{s}$ in (4.28) when $\phi=0^{\circ}$ such that

$$
f_{s}:=k_{s}\left(x_{2}-x_{1}-r_{s}\right)
$$

Moreover, we define the external environment force $F_{e}$ whose only non-zero component, $f_{e} \in \mathbb{R}$, acts along the $x_{0}$-axis. The force is non-zero when the second mass is in contact with 
the environment located at $\left(\hat{\rho}_{e}, 0\right)$, and is modelled as a virtual spring with spring constant $k_{e}$ attached between the second mass and the environment:

$$
f_{e}=\left\{\begin{array}{cc}
k_{e}\left(\hat{\rho}_{e}-x_{2}\right) & x_{2} \geq \hat{\rho}_{e} \\
0 & x_{2}<\hat{\rho}_{e}
\end{array}\right.
$$

It is assumed that the exact value of $k_{e}$ is unknown but that it is bounded within a known range given by $k_{e} \in\left[k_{e m i n}, k_{e m a x}\right]$.

Finally, let $u \in \mathbb{R}$ be the first component of $U$. Then, the equations of motion of interest are the $x$-dynamics of (4.23) and the first row of (4.32) (wherein since $\phi=0^{\circ}$ and motion is horizontal we assume that $\ddot{\phi} \simeq 0$ )

$$
\begin{aligned}
& m_{1} \ddot{x}_{1}=f_{s}+u \\
& m_{2} \ddot{x}_{2}=-f_{s}+f_{e} .
\end{aligned}
$$

Next, assumption (C2) allows the dynamics of the second mass to be simplified, and for the two-mass model (4.35) to be reduced to a single-mass model. In the case of no contact it is shown in [37] that when $m_{1}>>m_{2}$, that the relative acceleration of the two masses is approximately zero, and that the equations of motion reduce to an algebraic constraint on $f_{s}$

$$
f_{s}=f_{e}-m_{2} \ddot{x}_{1}
$$

Substitution of (4.36) into (4.35a) gives $m_{1} \ddot{x}_{1}=-m_{2} \ddot{x}_{1}+f_{e}+u$. Since there is no contact then $f_{e}=0$, and since $m_{1}>>m_{2}$ we obtain the single-mass free motion equation

$$
m_{1} \ddot{x}_{1}=u \text {. }
$$

For the case when there is contact with the environment we first solve (4.35b) for $x_{2}$ to get

$$
x_{2}=\frac{1}{k_{s}+k_{e}}\left[-m_{2} \ddot{x}_{2}+k_{s}\left(x_{1}+r_{s}\right)+k_{e} \hat{\rho}_{e}\right] \text {. }
$$


Next, assumption (C3) is applied to produce

$$
x_{2}=\frac{1}{k_{s}+k_{e}}\left[k_{s}\left(x_{1}+r_{s}\right)+k_{e} \hat{\rho}_{e}\right]
$$

Substituting (4.33) and (4.38) into (4.35a) and simplifying, we obtain

$$
m_{1} \ddot{x}_{1}=\bar{k}\left(\hat{\rho}_{e}-r_{s}-x_{1}\right)+u
$$

where

$$
\bar{k}:=\frac{k_{s} k_{e}}{k_{s}+k_{e}}
$$

is called the effective spring constant. Observe that since $k_{e} \in\left[k_{\text {emin }}, k_{\text {emax }}\right]$, then the effective spring constant will also vary as $\bar{k} \in\left[\bar{k}_{\text {min }}, \bar{k}_{\text {max }}\right]$, e.g. $k_{e}=k_{\text {emin }} \Longrightarrow \bar{k}=\bar{k}_{\text {min }}$.

Finally, we require a model for the dynamics of the sensed force so that a suitable force controller may be designed for force regulation during contact with the environment. First we substitute (4.38) into (4.33) and simplify to get

$$
f_{s}=\bar{k}\left(\hat{\rho}_{e}-r_{s}-x_{1}\right)
$$

Now take the second derivative of (4.40) and substitute the model (4.39) to obtain the secondorder force dynamics

$$
m_{1} \ddot{f}_{s}+\bar{k} f_{s}+\bar{k} u=0
$$

We are interested in designing a force controller to stabilize $f_{s}$ to some $f_{s}^{d}<0$. A suitable PD controller is

$$
u_{f}=-f_{s}+k_{1}\left(f_{s}-f_{s}^{d}\right)+k_{2} \dot{f}_{s}
$$

where parameters $k_{1}, k_{2}>0$ determine the closed-loop poles.

We can now summarize all the state space models that will be used for control design. Let $\xi=\left(x_{1}, \dot{x}_{1}\right)$. When there is no contact with the environment, the free motion of the first mass 
is given by

$$
\dot{\xi}=\left[\begin{array}{ll}
0 & 1 \\
0 & 0
\end{array}\right] \xi+\left[\begin{array}{c}
0 \\
\frac{1}{m_{1}}
\end{array}\right] u
$$

The output of this model is the position of the second mass given by

$$
x_{2}=\xi_{1}+r_{s}
$$

When there is contact with the environment, the single mass approximation of the two mass system is

$$
\dot{\xi}=\left[\begin{array}{ll}
0 & 1 \\
0 & 0
\end{array}\right] \xi+\left[\begin{array}{c}
0 \\
\frac{1}{m_{1}}
\end{array}\right] u+\left[\begin{array}{c}
0 \\
\frac{1}{m_{1}}
\end{array}\right] f_{s} .
$$

Based on (4.38), the output of this model is the position of the second mass given by

$$
x_{2}=\frac{1}{k_{s}+k_{e}}\left[k_{s}\left(\xi_{1}+r_{s}\right)+k_{e} \rho_{e}\right] .
$$

Next, by substituting (4.42) into (4.35a), we obtain the state model for the closed-loop force dynamics when there is contact with the environment

$$
\dot{\xi}=\left[\begin{array}{cc}
0 & 1 \\
-\frac{\bar{k} k_{1}}{m_{1}} & -\frac{\bar{k} k_{2}}{m_{1}}
\end{array}\right] \xi+\left[\begin{array}{c}
0 \\
\frac{\bar{k} k_{1} \rho_{f}^{d}}{m_{1}}
\end{array}\right],
$$

where, letting $\rho_{e}=\hat{\rho}_{e}-r_{s}$ and recalling that $f_{s}^{d}<0$ we define the position corresponding to the contact force $f_{s}^{d}$ as

$$
\rho_{f}^{d}:=\hat{\rho}_{e}-r_{s}-\frac{f_{s}^{d}}{\bar{k}}=\rho_{e}+\frac{\left|f_{s}^{d}\right|}{\bar{k}}
$$

System (4.43c) with output (4.43d) is the primary model for which a control scheme will be developed. It represents the motion of one large mass with a control input, and an opposing environmental force. Thus, we have reduced our initial problem of controlling a robotic manipulator with a mass at its end effector to controlling a single mass.

Lastly, we extract the dynamics of the remaining task space variables $y_{1}$ and $\phi$ from (4.23). Given the previous discussion, when $\phi$ is regulated to approximately zero we have that the 
second and third of components of $F_{s}$ will be approximately zero. Let $u_{y}, u_{\phi} \in \mathbb{R}$ be the second and third components of $U$. Then the remaining task space dynamics are given by the following

$$
m_{1} \ddot{y}_{1}=u_{y}, \quad m_{1} \ddot{\phi}=u_{\phi} .
$$

By construction, the output of the model for $y_{1}$ is $y_{2}$ of the second mass given by

$$
y_{2}=y_{1}
$$




\section{Chapter 5}

\section{Hybrid Reach/Force Control of Manipulators}

In this chapter we formulate and solve the problem of achieving a bumpless transfer from reach to force control using the principles of the Reach Control Problem. In the context of the state space models of Chapter 4, a set of logic control specifications are given that encode the desired system behaviour. The solution methodology is developed through several lemmas, and an algorithm is presented that provides the design steps laying out the selection of certain design parameters and the synthesis of reach controllers.

\section{$5.1 \quad$ Logic Control Specifications}

In this section we present the control problem to be solved. Since the problem is not formulated as a typical regulation-based control problem, we begin with a general non-technical discussion on our approach.

Consider an industrial scenario where a robot arm must reach out and make contact with an object herein called the environment. The nominal control goal may be described qualitatively as: the end effector travels in the direction of the environment, makes contact with the environment, and then remains in contact with the environment. This description, called the desired temporal sequence, dictates qualitatively the desired behaviour or sequence of events to be performed, but does not specify performance requirements. When the end effector is far 
from its desired destination we want to prevent it from idling. Thus, we include a performance requirement of sufficiently fast motion far from the goal. Such a requirement is called a liveness requirement; that is, a specification that drives the system to complete the control objective in a lively manner. Contrasting the liveness requirement is a set of safety requirements which prevent the system from violating technological limits or potentially causing harm. For the robot arm, the primary safety requirement is that at the moment of contact, the relative velocity between the arm and the environment must be below a threshold. The safety, liveness, and temporal sequence specifications are known collectively as logic control specifications.

We now discuss in general terms the problem formulation. We are given the system model (4.43a)-(4.43b), force controller (4.42) with closed-loop dynamics (4.43e), and the models (4.44a)-(4.44b). Also we are given the system parameters masses $m_{1}$ and $m_{2}$ with $m_{1}>>m_{2}$; sensed force parameters $k_{s}$ and $r_{s}$; the range for the environment spring constant, $k_{e} \in$ $\left[k_{\text {emin }}, k_{\text {emax }}\right]$; the range for the effective spring constant, $\bar{k} \in\left[\bar{k}_{\min }, \bar{k}_{\text {max }}\right]$; the range of the environment position $\hat{\rho}_{e} \in\left[\hat{\rho}_{\text {emin }}, \hat{\rho}_{\text {emax }}\right]$ where $0 \leq \hat{\rho}_{\text {emin }} \leq \hat{\rho}_{\text {emax }} ;$ parameters $\rho_{e}, \rho_{\text {emin }}, \rho_{\text {emax }}$ representing the aforementioned environment positions shifted back by $r_{s}$; and force controller parameters $k_{1}, k_{2}$, and $f_{s}^{d}$. We are also given two of the specification parameters from the logic control specifications. First, $\rho_{0}$, the minimum allowable position of the end effector is assumed

Table 5.1: System Parameters and Definitions.

\begin{tabular}{c|c} 
Parameter & Definition \\
\hline \hline$u_{f}$ & Force Controller \\
$\rho_{0}$ & Minimum allowable end effector position \\
$\mu_{1}$ & Maximum allowable end effector speed \\
$f_{s}^{d}$ & Reference contact force \\
$f_{s w}$ & Switching-force threshold \\
$\rho_{s w}$ & Switching-position threshold \\
$\Gamma_{s w}$ & Switching boundary \\
$\rho_{e}$ & Shifted environment position \\
$\rho_{e m i n}$ & Shifted minimum environment position \\
$\rho_{e m a x}$ & Shifted maximum environment position \\
$\rho_{1}$ & Position constraint parameter \\
$\rho_{2}$ & Maximum allowable end effector position \\
$\rho_{3}$ & Position constraint parameter \\
$\mu_{s w}$ & Switching velocity \\
$\mu_{2}$ & Velocity constraint parameter
\end{tabular}


to be given. Second, $\mu_{1}$, the maximum allowed velocity of the end effector is given. The overall control objective is to design a reach controller $u_{r c p}$ along with a switching logic for switching between $u_{r c p}$ and the given force controller $u_{f}$ to guarantee that all closed-loop trajectories satisfy the following behaviour: 1) Assuming that the first mass starts at an initial condition with $|\phi(0)|<90^{\circ}, \xi_{1}(0) \in\left[\rho_{0}, \rho_{\text {emin }}\right)$ corresponding to non-contact of the second mass, then under control of $u_{r c p}$ it reaches a position $\rho_{e}$ corresponding to the second mass making contact with the environment; 2) When the sensed force reaches a threshold $f_{s w}$, the force controller $u_{f}$ is activated and the sensed force is regulated to a desired reference value $\left.f_{s}^{d} ; 3\right)$ The primary safety requirement is that the velocity at impact may not exceed some value $\mu_{s w}$; 4) Finally, the second mass must maintain contact with the environment. That is, there is no switching or bouncing between force control and reach control once the force controller has been initiated.

It is necessary to distill this problem statement to a more concrete form which will allow a reach control problem to be formulated. In the present case, based on an analysis of the specific problem requirements and the features of the system dynamics, further specification parameters are required primarily in order to fix a polytopic state space on which the relevant dynamics evolve. How these additional specification parameters are selected is the content of Algorithm 5.1 presented in section 5.2. Here we give the basic idea in order to make sense of the problem statement to follow, which will have these additional specification parameters already built into it.

Additional specification parameters (besides simply $\rho_{0}, \mu_{1}$ ) are required to determine the polytopic state space. It is the shape of this polytopic state space that will guarantee that the no bouncing or no switching requirement between force control and reach control is met. This guarantee is the main theoretical contribution of the thesis. It is based on a Lyapunov analysis of the force controller and is the subject of Lemmas 5.2 and 5.3. The extra specification parameters are as follows. First is the parameter $\mu_{s w}$, which is related to the sensed force when switching occurs between reach control and force control. The parameter $\mu_{s w}$ is chosen to guarantee no bouncing as developed in Lemmas 5.2 and 5.3. The parameter is selected so that at the moment of switching from reach control to force control, trajectories of the closed-loop system are forced to enter an invariant set under the dynamics of the force controller. Second, a parameter $\rho_{2}$ is determined which is based on the switching force threshold $f_{s w}$ and the range 
of environment positions. This threshold can be translated to a position threshold, namely a position of the second mass at which a switch to force control occurs. The requirement on $\rho_{2}$ is that it be larger than this position for switching to force control. Next are parameters $\mu_{2}$ and $\rho_{1}$ which are determined so that the three points $\left(\rho_{1}, 0\right),\left(\rho_{2}, 0\right),\left(\rho_{1}, \mu_{2}\right)$ form a simplex with the requirement that $\rho_{1}<\rho_{\text {emin }}<\rho_{2}$. Finally, $\rho_{3}$ is the position at which the end effector must move back to when reattempting contact with the environment in case a bounce does occur. Additionally, the selection of $\rho_{3}$ affects in part the distance over which the manipulator will be made to decelerate from high to low velocity.

To summarize, in the problem statement below, both requirements to achieve a desired impact velocity and the no bouncing requirement are embedded in the selection of specification parameters $\mu_{s w}, \rho_{2}, \rho_{1}, \mu_{2}$ and $\rho_{3}$. These parameters will all be selected so that the system arrives at a switching boundary $\Gamma_{s w}$ with sufficiently small velocity so that a switch back out of force control cannot occur. From the point of view of reach control design, these parameters are viewed as being given; hence, their appearance in the specifications below.

The specifications will be presented in terms of the states $\xi$ of the first mass for which the model $(4.43 \mathrm{a})-(4.43 \mathrm{~b})$ is available. However, these $\xi$ requirements are then related to requirements on the states $\left(x_{2}, \dot{x}_{2}\right)$ of the second mass using the relationships

$$
\begin{aligned}
& x_{2}=\xi_{1}+r_{s} \\
& \dot{x}_{2}=\xi_{2} .
\end{aligned}
$$

In order to relate the position of the environment relative to the base frame to a position of the first mass, we formally define the following variables:

$$
\begin{aligned}
\rho_{\text {emin }} & :=\hat{\rho}_{\text {emin }}-r_{s} \\
\rho_{\text {emax }} & :=\hat{\rho}_{\text {emax }}-r_{s} \\
\rho_{e} & :=\hat{\rho}_{e}-r_{s} .
\end{aligned}
$$

For example, when the first mass is at $\rho_{e}$, the second mass contacts the environment at $\hat{\rho}_{e}$.

Finally, we include traditional regulation requirements for orientation angle $\phi$, and position 


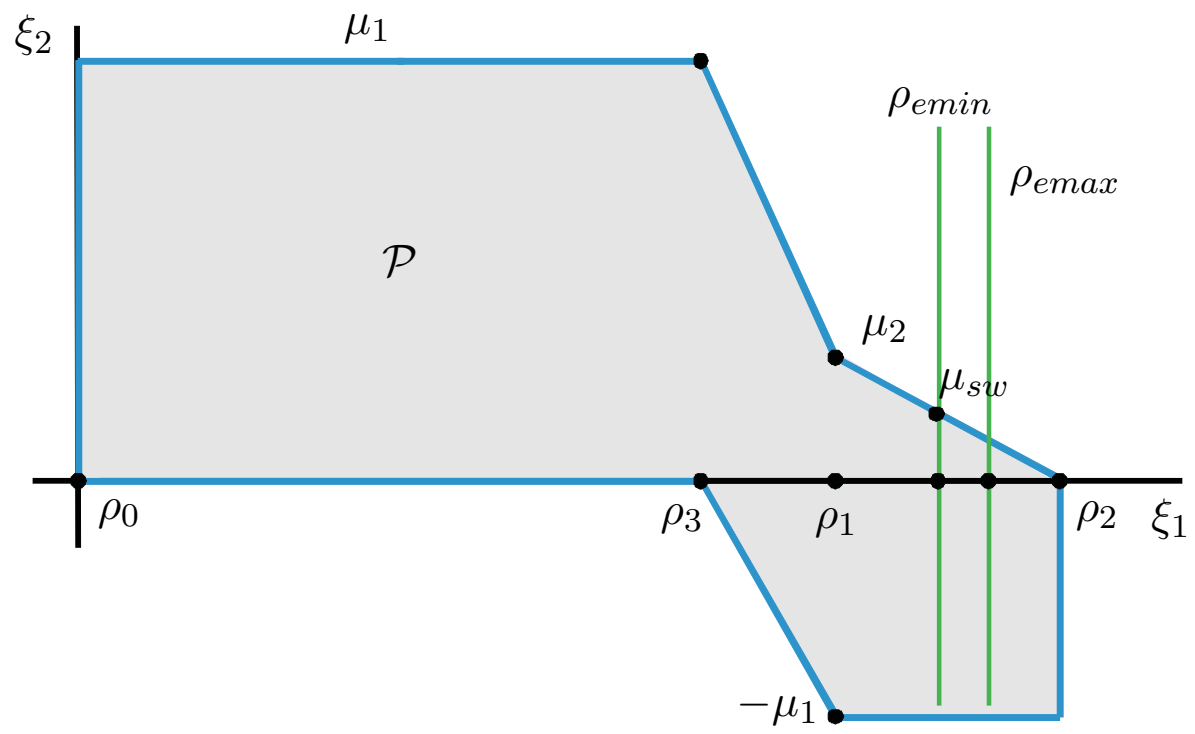

Figure 5.1: The state space $\mathcal{P}$ of $\left(\xi_{1}, \xi_{2}\right)$ according to the Logic Control Specifications.

coordinate $y_{1}$ of the first mass; these properties have no safety or liveness specifications but should meet some steady-state requirements. In particular, the orientation of the end effector will be regulated such that $\phi=0^{\circ}$ to induce a horizontal approach to the environment. Therefore, as with the $x$-direction, we translate the desired steady-state behaviour of $\left(y_{1}, \dot{y}_{1}\right)$ into that for $\left(y_{2}, \dot{y}_{2}\right)$ of the second mass by the relationship

$$
y_{2}=y_{1}, \quad \dot{y}_{2}=\dot{y}_{1}
$$

Problem 5.1. We consider the model (4.43a)-(4.43b) along with a force controller (4.42), and model (4.44a)-(4.44b). We are given the system parameters masses $m_{1}$ and $m_{2}$ with $m_{1}>>m_{2}$; sensed force parameters $k_{s}$ and $r_{s}$; the range for the environment spring constant, $k_{e} \in$ $\left[k_{\text {emin }}, k_{\text {emax }}\right] ;$ the range for the effective spring constant, $\bar{k} \in\left[\bar{k}_{\min }, \bar{k}_{\text {max }}\right]$; the range of the environment position $\hat{\rho}_{e} \in\left[\hat{\rho}_{\text {emin }}, \hat{\rho}_{\text {emax }}\right]$ where $0 \leq \hat{\rho}_{\text {emin }} \leq \hat{\rho}_{\text {emax }}$; and force controller parameters $k_{1}, k_{2}$, and $f_{s}^{d}$. Finally, we are given the specification parameters: $\rho_{i}, i=0, \ldots, 3, \mu_{1}$, $\mu_{2}, \mu_{s w}$. The measurements are the states of the first mass $\xi,\left(y_{1}, \dot{y}_{1}\right), \phi, \dot{\phi}$ the sensed force $f_{s}$ and its derivative $\dot{f}_{s}$. The control objective is to find a state feedback such that the closed-loop system achieves the following specifications. 


\section{(S1) Temporal Sequence}

- The first mass starts at an initial condition with $|\phi(0)|<90^{\circ}, \xi_{1}(0) \in\left[\rho_{0}, \rho_{\text {emin }}\right)$ corresponding to non-contact of the second mass. Eventually it reaches a position $\rho_{e}$, corresponding to the second mass making contact. Then the second mass maintains contact with the environment.

(S2) Safety

$-\rho_{0} \leq \xi_{1} \leq \rho_{2}$ where $\rho_{2}>\rho_{\text {emax }}$.

$-\left|\xi_{2}\right| \leq \mu_{1}$.

- If $\xi_{1} \in\left[\rho_{3}, \rho_{1}\right]$ and $\xi_{2} \geq 0$, then $\left(\mu_{1}-\mu_{2}\right) \xi_{1}+\left(\rho_{1}-\rho_{3}\right) \xi_{2} \leq \rho_{1} \mu_{1}-\rho_{3} \mu_{2}$.

- If $\xi_{1} \in\left[\rho_{1}, \rho_{2}\right]$ and $\xi_{2} \geq 0$, then $\mu_{s w} \xi_{1}+\left(\rho_{2}-\rho_{e m i n}\right) \xi_{2} \leq \mu_{s w} \rho_{2}$.

(S3) Liveness

- If $\xi_{1} \in\left[\rho_{0}, \rho_{3}\right]$, then $\xi_{2} \geq 0$.

- If $\xi_{1} \in\left[\rho_{3}, \rho_{1}\right]$ and $\xi_{2} \leq 0$, then $\mu_{1} \xi_{1}+\left(\rho_{1}-\rho_{3}\right) \xi_{2} \geq \mu_{1} \rho_{3}$.

(S4) Regulation

$-y_{1} \longrightarrow y_{1}^{d}$

$-\phi \longrightarrow 0^{\circ}$.

$-f_{s} \longrightarrow f_{s}^{d}$

(S1) conveys the desired temporal behaviour that the second mass approaches the environment whose exact position is unknown but is at a position between $\left[\hat{\rho}_{\text {emin }}, \hat{\rho}_{\text {emax }}\right]$ along the $x_{0}$-coordinate from the base of the manipulator. The first safety requirement of (S2) restricts the motion of the first mass along the $x_{0}$ coordinate: its position must be beyond the location of joint 1 at $\rho_{0}$, the first pitch joint at the base frame, and no further than some point $\rho_{2}>\rho_{\text {emax }}$. A singularity occurs upon full extension, i.e. $\theta_{2}=0$, so $\rho_{2}$ is chosen such that the manipulator will not be fully extended if this point is reached. The second safety requirement restricts the maximum velocity of mass one. The third and fourth safety requirements comprise linear inequalities in terms of $\left(\xi_{1}, \xi_{2}\right)$ that are used to restrict the admissible velocity as a function of 
position. Specifically, the third safety specification corresponds to the diagonal edge above the $\xi_{1}$ axis connecting the vertices $\left(\rho_{3}, \mu_{1}\right)$ and $\left(\rho_{1}, \mu_{2}\right)$, as depicted in Figure 5.1. This specification imposes a quick deceleration of the manipulator as it nears the environment. The rate of deceleration depends on the slope of this diagonal edge. Next, the fourth safety specification corresponds to the diagonal edge that connects the vertices $\left(\rho_{1}, \mu_{2}\right)$ and $\left(\rho_{2}, 0\right)$. This specification effectively restricts the maximum allowed relative velocity at impact between the second mass and the environment. In particular, assuming that the environment is stationary but lies at a distance between $\left[\hat{\rho}_{\text {emin }}, \hat{\rho}_{\text {emax }}\right]$, the maximum relative velocity at impact can be derived from the fourth safety constraint by solving for $\xi_{2}$ to obtain

$$
\xi_{2} \leq \mu_{s w} \frac{\rho_{2}-\xi_{1}}{\rho_{2}-\rho_{e m i n}}
$$

It is evident from the formula that the maximum relative velocity at impact occurs when the first mass is at $\rho_{\text {emin }}$; correspondingly the environment is at $\hat{\rho}_{\text {emin }}$. Setting $\xi_{1}=\rho_{\text {emin }}$ we get a maximum relative velocity of the first mass at impact of $\mu_{s w}$. The first liveness requirement of (S3) forces the first mass to move toward the environment in a lively way when it is sufficiently far from the environment. The second liveness requirement, which describes the diagonal edge connecting $\left(\rho_{3}, 0\right)$ and $\left(\rho_{1},-\mu_{1}\right)$, states that the first mass may move backwards, away from the environment, but only as far back as $\rho_{3}$. This requirement allows the system to approach the environment again, while limiting how far back it may travel.

Of course other more complex or refined safety and liveness inequality constraints could have been chosen. However, as the complexity of the specification increases, so too does the complexity of the control design. The selected constraints are sufficiently simple to make the control design reasonable but also achieve the desired ends of the problem.

\subsection{Main Results}

In this section we present the methodology to solve Problem 5.1. We work with the models (4.43a) - (4.43f) to achieve the specifications in terms of the states $\xi$, and models (4.44a)-(4.44b) for $y_{1}$ and $\phi$. 


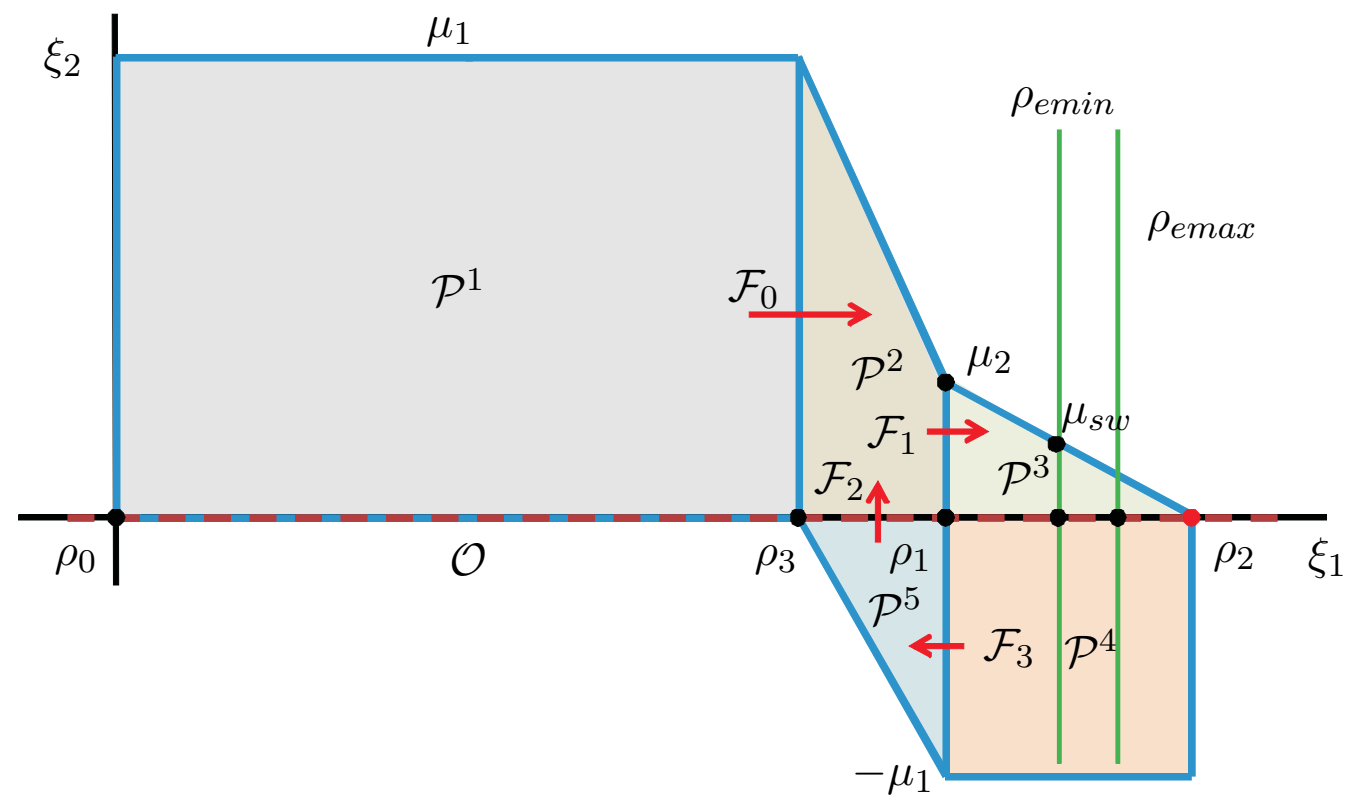

Figure 5.2: The subdivision of $\mathcal{P}$ and intended directions of travel across exit facets $\mathcal{F}_{i}$.

First we briefly address the regulation specification from (S4), which comprises a simple set-point tracking problem. The standard PD control law can be used to regulate $y_{1}$ and $\phi$ as

$$
\begin{aligned}
& u_{y}=m_{1} k_{p y}\left(y_{1}^{d}-y_{1}\right)+m_{1} k_{d y}\left(0-\dot{y_{1}}\right), \\
& u_{\phi}=m_{1} k_{p \phi}(0-\phi)+m_{1} k_{d \phi}(0-\dot{\phi}) .
\end{aligned}
$$

It is assumed that the regulation of $\left(y_{1}, \phi\right)$ occurs sufficiently quick that horizontal motion towards the environment is achieved prior to contact. We now give an outline and basic intuition of the control design and main contributions of the thesis.

The theoretical work of this section can be categorized into two objectives: 1) Obtain the unknown constraint specifications that determine a state space $\mathcal{P}$ corresponding to bumpless transfer; 2) Design a control under which the system dynamics evolve through $\mathcal{P}$ to achieve bumpless transfer. The first objective requires the specification of parameters $\mu_{s w}, \rho_{2}, \mu_{2}, \rho_{1}$, and $\rho_{3}$ as defined in Table 5.1. The value of $\mu_{s w}$ is found via Lyapunov analysis of the force dynamics and a switching logic, and is discussed in Lemmas 5.2 and 5.3. In Lemma 5.2 a Lyapunov function is selected for the model (4.43e) via a symmetric, positive definite matrix $P$. Then, an invariant set is shown to exist within a sub-level of the Lyapunov function. Part of the boundary of the invariant set is the line segment delimited by the points $\left(\rho_{s w}, 0\right)$ and 
$\left(\rho_{s w}, \mu_{s w}\right)$, where $\rho_{s w}$ is the position corresponding to the switching force $f_{s w}$. Therefore, the conditions $\xi_{1}=\rho_{s w}$, and $0 \leq \xi_{2} \leq \mu_{s w}$ form the basis of a switching logic for achieving bumpless transfer.

This result is valid assuming a single environment stiffness. In order to extend the result to hold for all $k_{e}$ we acknowledge an important implication from Lemma 5.2. Namely, that given $P$ we obtain $\mu_{s w}$, which corresponds to a boundary of an invariant set delimited by the points $\left(\rho_{s w}, 0\right)$ and $\left(\rho_{s w}, \mu_{s w}\right)$. What we seek instead is the opposite implication whereby a switching velocity $\mu_{s w}^{d}$ is selected, which, for any $k_{e}$, can be shown to correspond to a similar invariant set as in Lemma 5.2. However, it is not immediately clear the values of $\mu_{s w}^{d}$ that one may select that pertain to such invariant sets. We seek then an explicit expression for $\mu_{s w}$ from which a range of "admissible" values may be extracted. The development that precedes Lemma 5.3 produces this expression through additional Lyapunov analysis and taking the limit of certain parameters. Ultimately, it is shown that $\mu_{s w}$ may range over $\mu_{s w} \in\left(0, \bar{\mu}_{s w}\right)$, where the velocity $\bar{\mu}_{s w}$ is the least-upper bound of $\mu_{s w}$. The expression for $\bar{\mu}_{s w}$ contains parameters associated with the given force controller and the proposed switched-system architecture. Therefore, based on the solution method, $\bar{\mu}_{s w}$ constitutes a characteristic property of the system. Using this fact, one may select a preferred positive definite matrix $Q^{d}$, whose elements depend on $\bar{\mu}_{s w}$ and $\mu_{s w}^{d}$. Then, given the Hurwitz system matrix $A$, a suitable matrix $P$ may be obtained by solving for $P$ in Lyapunov's equation $A^{T} P+P A=-Q^{d}$. The $P$ matrix is then used to define a quadratic Lyanpuov function. This procedure is used in Lemma 5.3 to show that for any velocity $\mu_{s w}^{d} \in\left(0, \bar{\mu}_{s w}\right)$ and any $k_{e}$ in its given range, that an invariant set exists, which when entered, ensures that a switch back to reach control can not occur. Necessarily, the set contains a boundary segment characterized by velocities in the range $\left[0, \mu_{s w}^{d}\right]$. This result yields a control design flow whereby a designer directly computes $\bar{\mu}_{s w}$ from known parameters, and then chooses a desired switching velocity $\mu_{s w}$ knowing that $\mu_{s w}$ is considered "safe".

Next, the parameters $\rho_{2}, \mu_{2}$, and $\rho_{1}$ are required to define a region in which contact with the environment is made. The parameter $\rho_{2}$ is defined in such a way that the manipulator states are guaranteed to remain in $\mathcal{P}$ at least up until the point that a switch to force control is made. Next are parameters $\mu_{2}$ and $\rho_{1}$ which are determined so that the three points $\left(\rho_{1}, 0\right),\left(\rho_{2}, 0\right)$, $\left(\rho_{1}, \mu_{2}\right)$ form a simplex with the requirement that $\rho_{1}<\rho_{\text {emin }}<\rho_{2}$. Note that in this simplex 
the manipulator velocity will be forced to decrease as the environment is approached. Finally, $\rho_{3}$ is the position at which the end effector must move back to when reattempting contact with the environment in case a loss of contact occurs. Additionally, $\rho_{3}$ affects in part the distance over which the manipulator will be made to decelerate from high to low velocity.

The above method for choosing these parameters is summarised in Algorithm 5.1, which when executed completes the numerical definition of the logic control specifications that encode bumpless transfer behaviour. The behaviour is guaranteed over the range of environment stiffness and location. Then, the safety and liveness constraints of the logic control specifications determine the sought after polytopic state space $\mathcal{P}$ shown in Figure 5.1. The desired temporal sequence determines how closed-loop trajectories must move through $\mathcal{P}$. The overarching requirement is that trajectories move through $\mathcal{P}$ from the left to the right and reach an environment corresponding to a vertical strip in the state space. Upon making contact with the environment, a force controller is invoked, and the requirement of the desired temporal sequence is that the manipulator must remain under force control and in contact with the environment.

With respect to the second objective of this section, our solution approach will be to divide the state space into five regions, $\mathcal{P}^{i}, i=1, \ldots, 5$, as seen in Figure 5.2 and to specify on each region a suitable closed-loop behaviour to achieve the overall specification. The closed-loop behaviour of each region will be achieved using a piecewise affine feedback based on the theory of reach controllers.

Referring to Figure 5.2, in region $\mathcal{P}^{1}$ a piecewise affine reach controller will be specified using the theory of Section 3.5 so that all trajectories initialized in $\mathcal{P}^{1}$ reach the exit facet $\mathcal{F}_{0}$ in finite time, corresponding to quick movement of the manipulator toward the environment. Borrowing the shorthand notation for RCP, we want $\mathcal{P}^{1} \stackrel{\mathcal{P}^{1}}{\longrightarrow} \mathcal{F}_{0}$. In region $\mathcal{P}^{2}$, whose shape corresponds to a rapid deceleration of the manipulator, a piecewise affine reach controller will be designed so that $\mathcal{P}^{2} \stackrel{\mathcal{P}^{2}}{\longrightarrow} \mathcal{F}_{1}$. The region $\mathcal{P}^{3}$, a simplex, will receive special treatment, detailed in Lemma 5.1. Here a reach controller will be specified to drive trajectories to reach a set of states corresponding to contact with the environment, after which a force controller is activated. Then in Lemma 5.2 it is shown that the closed-loop system (4.43e) under the force controller (4.42) can never return to a state corresponding to a reach control modality. In region $\mathcal{P}^{4}$ a piecewise affine reach controller will be designed so that $\mathcal{P}^{4} \stackrel{\mathcal{P}^{4}}{\longrightarrow} \mathcal{F}_{3}$ and similarly on $\mathcal{P}^{5}$ 
we require $\mathcal{P}^{5} \stackrel{\mathcal{P}^{5}}{\longrightarrow} \mathcal{F}_{2}$. Region $\mathcal{P}^{5}$ corresponds to the manipulator decelerating away from the environment in order to set up another approach to it in region $\mathcal{P}^{2}$. Again, the combination of the reach controllers, proposed switching logic, and given force controller constitute an overall hybrid control that oversees the interaction task. This control is provided at the end of this section along with the main theorem that summarises the overall system behaviour.

We proceed now with the theoretical results. Specifically, the following three lemmas describe the main features of the control design for $\mathcal{P}^{3}$, and the solution to the bouncing problem. First we place an assumption on the parameters of the force controller (4.42).

Assumption 5.1. For the force controller (4.42), we assume $k_{1}$ and $k_{2}$ are such that for all $\bar{k} \in\left[\bar{k}_{\text {min }}, \bar{k}_{\text {max }}\right]$,

$$
A:=\left[\begin{array}{rr}
0 & 1 \\
-\frac{\bar{k} k_{1}}{m_{1}} & -\frac{\bar{k} k_{2}}{m_{1}}
\end{array}\right]
$$

is Hurwitz.

Note that Assumption 5.1 is met if $k_{1}, k_{2}>0$. Now suppose that a switching force $f_{s w} \leq 0$ is specified corresponding to when force control must be initiated. Let $\rho_{s w}$ be the position of the first mass corresponding to a sensed force of $f_{s}=f_{s w}$ under the dynamics (4.43c). This position (in analogy with (4.43f) relating the desired force and its corresponding position) is given by

$$
\rho_{s w}:=\rho_{e}+\frac{\left|f_{s w}\right|}{\bar{k}}
$$

Observe that by definition $\rho_{s w} \geq \rho_{e}$, so the switching position corresponds to mass two being in contact with the environment. We also define the switching boundary $\Gamma_{s w}$ in $\mathcal{P}^{3}$ by

$$
\Gamma_{s w}:=\left\{\xi \in \mathcal{P}^{3} \mid \xi_{1}=\rho_{s w}\right\}
$$

Let $\mathcal{P}^{3}=\operatorname{co}\left\{v_{0}, v_{1}, v_{2}\right\}$ where $v_{0}=\left(\rho_{1}, \mu_{2}\right), v_{1}=\left(\rho_{1}, 0\right)$, and $v_{2}=\left(\rho_{2}, 0\right)$, where $\rho_{1}<\rho_{2}$ and $\mu_{2}>0$. Also, the environment position lies in $\mathcal{P}^{3}$; that is, $\rho_{1}<\rho_{\text {emin }} \leq \rho_{e} \leq \rho_{\text {emax }}<\rho_{2}$. See Figure 5.3. Our first goal is to construct an affine reach controller for $\mathcal{P}^{3}$ such that when there is no contact with the environment, closed-loop trajectories of the system (4.43a) are driven to an equilibrium at $v_{2}$. Second, using the same reach controller but after contact is made with 

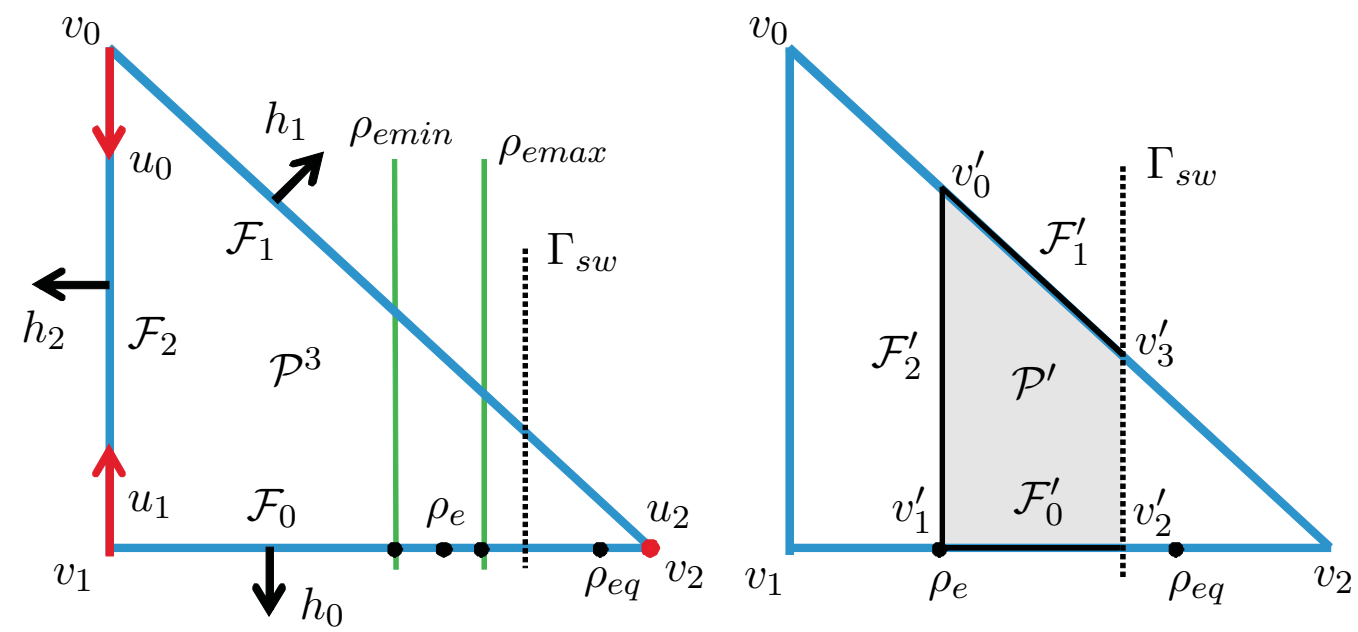

Figure 5.3: Salient variables for region $\mathcal{P}^{3}$, and the polytope $\mathcal{P}^{\prime}$ in the proof of Lemma 5.1.

the environment, all closed-loop trajectories of the system $(4.43 \mathrm{c})$ reach $\Gamma_{s w}$. To that end, we choose

$$
\rho_{2}>\rho_{\text {emax }}+\frac{\left|f_{s w}\right|}{\bar{k}_{\text {min }}} \geq \rho_{s w}
$$

Since $v_{2}=\left(\rho_{2}, 0\right)$, this places $v_{2}$ to the right of $\rho_{\text {emax }}$. Then since the reach controller drives trajectories of $(4.43 \mathrm{a})$ to $v_{2}$, contact will be made with the environment and moreover, a sufficiently high contact force is induced to allow states to reach $\Gamma_{s w}$.

Lemma 5.1. Consider the simplex $\mathcal{P}^{3}$ and systems $(4.43 \mathrm{a})-(4.43 \mathrm{~b})$, where $\bar{k} \in\left[\bar{k}_{\text {min }}, \bar{k}_{\text {max }}\right]$, and $\hat{\rho}_{e} \in\left[\hat{\rho}_{\text {emin }}, \hat{\rho}_{\text {emax }}\right]$. There exists an affine feedback $u=K \xi+g$ such that:

(i) For system (4.43a), the closed-loop trajectories satisfy: for all $\xi_{0} \in \mathcal{P}^{3}, \phi\left(t, \xi_{0}\right) \in \mathcal{P}^{3}$ for all $t \geq 0$. Moreover, $\lim _{t \rightarrow \infty} \phi\left(t, \xi_{0}\right)=v_{2}$.

(ii) For system (4.43c), the closed-loop trajectories satisfy: for all $\xi_{0} \in\left\{\xi \in \mathcal{P}^{3} \mid \rho_{e}<\xi_{1}<\right.$ $\left.\rho_{s w}\right\}, \phi\left(t, \xi_{0}\right)$ reaches $\Gamma_{s w}$ in finite time.

Proof. First we prove (i). Let the vertices of $\mathcal{P}^{3}$ be given by $v_{0}=\left(\rho_{1}, \mu_{2}\right), v_{1}=\left(\rho_{1}, 0\right)$, and $v_{2}=\left(\rho_{2}, 0\right)$. We select control values at the vertices of $\mathcal{P}^{3}$ satisfying $u_{0}<0, u_{2}=0$, and

$$
u_{1}>\frac{\left|f_{s w}\right|\left(\rho_{2}-\rho_{1}\right)}{\left(\rho_{2}-\rho_{e \max }-\frac{\left|f_{s w}\right|}{\bar{k}_{\min }}\right)}>0 .
$$

The particular form of (5.6) will be explained in the proof of (ii) below. Using (3.10) one 
can synthesize the affine feedback $u=K \xi+g$. First we show that under the feedback $u$ the invariance conditions (3.4) hold on each vertex, such that none of the $\mathcal{F}_{i}$ are exit facets. To that end, let $h_{i x}, h_{i y}$ be the components that comprise the normal vectors $h_{i} \in \mathbb{R}^{2}$ of facets $\mathcal{F}_{i}$. Then, starting with $\mathcal{F}_{0}$ we have

$$
\begin{aligned}
& h_{0} \cdot\left(A v_{1}+B\left(K v_{1}+g\right)+a\right)=h_{0} \cdot\left(A v_{1}+B u_{1}+a\right)=(0,-1) \cdot\left(0, \frac{u_{1}}{m_{1}}\right) \leq 0 \Longrightarrow u_{1} \geq 0 . \\
& h_{0} \cdot\left(A v_{2}+B\left(K v_{2}+g\right)+a\right)=h_{0} \cdot\left(A v_{2}+B u_{2}+a\right)=(0,-1) \cdot(0,0) \leq 0 .
\end{aligned}
$$

For $\mathcal{F}_{1}$ we have

$$
\begin{aligned}
& h_{1} \cdot\left(A v_{0}+B\left(K v_{0}+g\right)+a\right)=h_{1} \cdot\left(A v_{0}+B u_{0}+a\right)=\left(h_{1 x}, h_{1 y}\right) \cdot\left(\mu_{2}, \frac{u_{0}}{m_{1}}\right) \leq 0 \Longrightarrow u_{0} \leq-m_{1} \mu_{2} \frac{h_{1 x}}{h_{1 y}}<0 . \\
& h_{1} \cdot\left(A v_{2}+B\left(K v_{2}+g\right)+a\right)=h_{1} \cdot\left(A v_{2}+B u_{2}+a\right)=\left(h_{1 x}, h_{1 y}\right) \cdot(0,0) \leq 0 .
\end{aligned}
$$

Lastly for $\mathcal{F}_{2}$ we have

$$
\begin{aligned}
& h_{2} \cdot\left(A v_{0}+B\left(K v_{0}+g\right)+a\right)=h_{2} \cdot\left(A v_{0}+B u_{0}+a\right)=(-1,0) \cdot\left(\mu_{2}, \frac{u_{0}}{m_{1}}\right) \leq 0 \\
& h_{2} \cdot\left(A v_{1}+B\left(K v_{1}+g\right)+a\right)=h_{2} \cdot\left(A v_{1}+B u_{1}+a\right)=(-1,0) \cdot\left(0, \frac{u_{1}}{m_{1}}\right) \leq 0 .
\end{aligned}
$$

By convexity of the closed-loop vector field, the above conditions imply that the vector field points inside $\mathcal{P}^{3}$ at all points on its boundary. It follows by a standard argument that for all $\xi_{0} \in \mathcal{P}^{3}, \phi\left(t, \xi_{0}\right) \in \mathcal{P}^{3}$ for all $t \geq 0$. Evaluating the closed-loop system at the point $v_{2}$, one immediately verifies it is an equilibrium of the closed-loop system. Now we apply Theorem 3.4 to show that all trajectories tend to $v_{2}$. There are two requirements ${ }^{1}$ : (a) the vector field points inside $\mathcal{P}^{3}$ at all points on its boundary; (b) the closed-loop velocity vectors at the vertices of $\mathcal{P}^{3}$ span $\mathbb{R}^{2}$. We have already verified (a). For (b), we have $A v_{0}+B u_{0}+a=\left(\mu_{2}, \frac{u_{0}}{m_{1}}\right)$ and $A v_{1}+B u_{1}+a=\left(0, \frac{u_{1}}{m_{1}}\right)$, which span $\mathbb{R}^{2}$. We conclude that $\lim _{t \rightarrow \infty} \phi\left(t, \xi_{0}\right)=v_{2}$.

Second we prove (ii). First we show that with the choice (5.6) the closed-loop system (4.43c) has only one equilibrium in $\mathcal{P}^{3}$ at $\xi_{e q}:=\left(\rho_{e q}, 0\right) \in \mathcal{P}^{3}$ where $\rho_{s w}<\rho_{e q}<\rho_{2}$. To that end, let $u=K \xi+g$ be the affine feedback synthesized in (i) where $K=\left[\begin{array}{ll}K_{1} & K_{2}\end{array}\right]$; it can be shown it.

${ }^{1}$ Theorem 3.4 actually gives three conditions, but one of them, that $v_{2} \in \mathcal{O}$, is automatically met, so we omit 
that $K_{1}<0$ and $g=-K_{1} \rho_{2}$. First, we express the dynamics of (4.43c) explicitly in terms of the sensed force $f_{s}$ and feedback $u$. Substitute (4.40) for $f_{s}$, and $u=K_{1} \xi_{1}+K_{2} \xi_{2}-K_{1} \rho_{2}$ into (4.43c) to obtain

$$
\left[\begin{array}{l}
\dot{\xi}_{1} \\
\dot{\xi}_{2}
\end{array}\right]=\left[\begin{array}{cc}
0 & 1 \\
\frac{\left(K_{1}-\bar{k}\right)}{m_{1}} & \frac{K_{2}}{m_{1}}
\end{array}\right]\left[\begin{array}{l}
\xi_{1} \\
\xi_{2}
\end{array}\right]+\left[\begin{array}{c}
0 \\
\frac{\bar{k} \rho_{e}-K_{1} \rho_{2}}{m_{1}}
\end{array}\right] .
$$

Next, setting $\dot{\xi}=(0,0)$ in $(5.7)$ one can solve for the equilibrium solution $\xi_{e q}=\left(\rho_{e q}, 0\right)$ where

$$
\rho_{e q}:=\frac{\bar{k}}{\bar{k}-K_{1}} \rho_{e}-\frac{K_{1}}{\bar{k}-K_{1}} \rho_{2}
$$

We now prove that $\rho_{s w}<\rho_{e q}<\rho_{2}$ over the variation of environment location $\rho_{e} \in\left[\rho_{e m i n}, \rho_{e m i n}\right]$ and stiffness $\bar{k} \in\left[\bar{k}_{\text {min }}, \bar{k}_{\text {max }}\right]$. We begin with $\rho_{e q}<\rho_{2}$. Let $c_{1}:=\frac{\bar{k}}{\bar{k}-K_{1}}$. Using $c_{1}$ we rewrite (5.8) as $\rho_{e q}=c_{1} \rho_{e}+\left(1-c_{1}\right) \rho_{2}$. Since $K_{1} \in(-\infty, 0)$ and $\bar{k} \in\left[\bar{k}_{\text {min }}, \bar{k}_{\text {max }}\right]$ then $c_{1} \in(0,1)$. As a result $\rho_{e q} \in\left(\rho_{e}, \rho_{2}\right)$. This relationship between $\rho_{e}, \rho_{e q}$, and $\rho_{2}$ holds irrespective of the values of $\bar{k}$ and $\rho_{e}$. Therefore $\rho_{e q}<\rho_{2}$.

We now prove that $\rho_{s w}<\rho_{e q}$. Using (5.8) for $\rho_{e q}$ and (5.3) for $\rho_{s w}$ we proceed to isolate for $-K_{1}$ :

$$
\begin{aligned}
\rho_{s w} & <\rho_{e q} \\
\rho_{e}+\frac{\left|f_{s w}\right|}{\bar{k}} & <\frac{\bar{k}}{\bar{k}-K_{1}} \rho_{e}-\frac{K_{1}}{\bar{k}-K_{1}} \rho_{2} \\
\frac{\left|f_{s w}\right|}{\rho_{2}-\rho_{e}-\frac{\left|f_{s w}\right|}{\bar{k}}} & <-K_{1} .
\end{aligned}
$$

Assuming the selection of controls at the vertices $u_{0}, u_{1}$, and $u_{2}$ from (i), the expression for $K_{1}$ from the synthesis of $u=K \xi+g$ using (3.10) is given by

$$
K_{1}=-\frac{u_{1}-u_{2}}{\rho_{2}-\rho_{1}}
$$

Using $u_{2}=0$ we substitute (5.9) into the inequality above, and rearrange terms to obtain

$$
\frac{\left|f_{s w}\right|\left(\rho_{2}-\rho_{1}\right)}{\rho_{2}-\rho_{e}-\frac{\left|f_{s w}\right|}{\bar{k}}}<u_{1}
$$


Finally, using the fact that $\rho_{e} \leq \rho_{\text {emax }}$, and $\bar{k} \geq \bar{k}_{\text {min }}$, we maximize the denominator in (5.10)

$$
\frac{\left|f_{s w}\right|\left(\rho_{2}-\rho_{1}\right)}{\rho_{2}-\rho_{\text {emax }}-\frac{\left|f_{s w}\right|}{\bar{k}_{\text {min }}}}<u_{1}
$$

The result is a minimum bound on the control $u_{1}$ that matches (5.6). We conclude that $\rho_{s w}<\rho_{e q}$, and along with the earlier result $\rho_{s w}<\rho_{e q}<\rho_{2}$.

Next in the proof of (ii), define the polytope $\mathcal{P}^{\prime}:=\operatorname{co}\left\{v_{0}^{\prime}, v_{1}^{\prime}, v_{2}^{\prime}, v_{3}^{\prime}\right\} \subset \mathcal{P}^{3}$ as depicted in Figure 5.3. The vertices are given by $v_{0}^{\prime}=\left(\rho_{e}, \mu_{e}\right), v_{1}^{\prime}=\left(\rho_{e}, 0\right), v_{2}^{\prime}=\left(\rho_{s w}, 0\right)$, and $v_{3}^{\prime}=$ $\left(\rho_{s w}, \mu_{s w}\right)$. Notice that $\Gamma_{s w}$ forms the right edge of $\mathcal{P}^{\prime}$. Define the facets of $\mathcal{P}^{\prime}$ to be $\mathcal{F}_{0}^{\prime}=$ $\operatorname{co}\left\{v_{1}^{\prime}, v_{2}^{\prime}\right), \mathcal{F}_{1}^{\prime}=\operatorname{co}\left\{v_{0}^{\prime}, v_{3}^{\prime}\right\}$, and $\mathcal{F}_{2}^{\prime}=\operatorname{co}\left\{v_{0}^{\prime}, v_{1}^{\prime}\right\}$. We now show that the closed-loop vector field for $(4.43 \mathrm{c})$ using the affine feedback given in part (i) points inward on $\mathcal{F}_{0}^{\prime}, \mathcal{F}_{1}^{\prime}$, and $\mathcal{F}_{2}^{\prime}$. Starting with $\mathcal{F}_{2}^{\prime}$, we observe that $\xi \in \mathcal{F}_{2}^{\prime}$ implies that $\xi_{1}=\rho_{e}$, and so $f_{s}\left(\rho_{e}\right)=0$ (see (4.40)); thus on $\mathcal{F}_{2}^{\prime}$ the closed-loop vector field for $(4.43 \mathrm{c})$ is equivalent to that for (4.43a). In (i) it was shown that the vector field points into $\mathcal{P}^{3}$ on its boundary, moreover that trajectories tend to $v_{2}$ for $t \geq 0$. Therefore on $\mathcal{F}_{2}^{\prime}$ the vector field points inward of $\mathcal{P}^{\prime}$. For $\mathcal{F}_{1}^{\prime}$ we know that the invariance conditions at $v_{0}^{\prime}$ holds, so we check that the condition at $v_{3}^{\prime}$ holds, i.e. that the dot product is non-positive. The condition can be decomposed into two parts:

$$
\left.h_{1} \cdot\left(A v_{3}^{\prime}+B\left(K v_{3}^{\prime}+g\right)\right)+h_{1} \cdot\left[\begin{array}{c}
0 \\
\frac{1}{m_{1}}
\end{array}\right] \bar{k}\left(\rho_{e}-\rho_{s w}\right)\right) .
$$

In the left-hand term, the dynamics $\left(A v_{3}^{\prime}+B\left(K v_{3}^{\prime}+g\right)\right)$ are again equivalent to those of (4.43a), and so it is known that this dot product is less than zero. The right-hand term accounts for the additional dynamics of (4.43c) induced by contact. Since $\rho_{e}<\rho_{s w}$, computing this dot product generates $\frac{h_{1 y} \bar{k}}{m_{1}}\left(\rho_{e}-\rho_{s w}\right)<0$ such that the sum $(5.12)$ is less than zero. Note that by definition $f_{s}\left(\xi_{1}\right)$ is a convex function as it is strictly linear in $\xi_{1}$ and monotonically decreases (thus we have $f_{s}(\xi) \in\left[-\bar{k}\left(\rho_{s w}-\rho_{e}\right), 0\right]$ for $\left.\xi_{1} \in \mathcal{F}_{1}^{\prime}\right)$. Therefore, by convexity the closed-loop vector field points inward on $\mathcal{F}_{1}^{\prime}$. Lastly for $\mathcal{F}_{0}^{\prime}$ we check the velocity vector at the remaining vertex $v_{2}^{\prime}$. Let $y(\xi)$ represent the closed-loop velocity vector at $\xi$ for dynamics (4.43c) under $u$. Using the fact that $f_{s}\left(\xi_{1}\right)$ is convex, then the closed-loop velocity at $v_{2}^{\prime}$ may be expressed as a 
convex combination of that at $v_{1}^{\prime}$ and $\xi_{\text {eq }}$, i.e. there exists $c_{1} \in(0,1)$ such that

$$
y\left(v_{2}^{\prime}\right)=c_{1} y\left(v_{1}^{\prime}\right)+\left(1-c_{1}\right) y\left(\xi_{e q}\right) .
$$

Since $y\left(\xi_{e q}\right)=(0,0)$, and since $y\left(v_{1}^{\prime}\right)$ has been shown to point into $\mathcal{P}^{\prime}$, then necessarily $y\left(v_{2}^{\prime}\right)$ points into $\mathcal{P}^{\prime}$. Similarly for all $\xi \in \mathcal{F}_{0}^{\prime}$.

Next, because $\xi_{\text {eq }} \notin \mathcal{P}^{3}$, it can be readily shown that $0 \notin \operatorname{co}\left\{A v_{1}^{\prime}+B\left(K v_{1}^{\prime}+g\right)+\mathbf{0}, A v_{2}^{\prime}+\right.$ $\left.B\left(K v_{2}^{\prime}+g\right)+\frac{1}{m_{1}}\left[\begin{array}{ll}0 & f_{s}\left(v_{2}^{\prime}\right)\end{array}\right]^{T}\right\}$ (in fact this was just shown above). Since $\mathcal{O}=\left\{\xi \in \mathbb{R}^{2} \mid \xi_{2}=0\right\}$, we can apply Theorem 3.10 to conclude that all trajectories in $\mathcal{P}^{\prime}$ reach $\Gamma_{s w}$ in finite time.

In the next lemma we show that for the system (4.43e) in contact with the environment, if the force controller (4.42) is initiated from states in $\mathcal{P}^{3}$ along the switching boundary $\Gamma_{s w}$, then under the controller (4.42) it is not possible for the state to return to a value with $\xi_{1}<\rho_{s w}$ (although it is permitted for the state to exit $\mathcal{P}^{3}$ ). In particular, this means that the system cannot switch back to a reach control modality. Because $\rho_{s w}$ will by definition correspond to contact with the environment, namely $\rho_{e} \leq \rho_{s w}$, it means the system remains in constant contact with the environment after the force controller is initiated. More specifically, the lemma shows that one can specify a segment of initial conditions in $\mathcal{P}^{3}$ corresponding to position $\xi_{1}=\rho_{s w}$ from which closed-loop trajectories will enter an invariant set for which $\xi_{1} \geq \rho_{s w}$ for all future time. We begin with a simpler result that shows that if $\rho_{s w}$ is fixed and known, then a maximum velocity $\mu_{s w}$ and set of initial conditions can be found to guarantee that trajectories do not return to a condition with $\xi_{1}<\rho_{s w}$.

Lemma 5.2. Consider system (4.43e) with $\rho_{f}^{d}>0, \bar{k} \in\left[\bar{k}_{\min }, \bar{k}_{\text {max }}\right]$, and $\rho_{e} \in\left[\rho_{\text {emin }}, \rho_{\text {emax }}\right]$. Suppose that $\rho_{e}<\rho_{s w}<\rho_{f}^{d}$. There exists $\mu_{s w}>0$ and a set of initial conditions

$$
\Omega_{0}:=\left\{\xi \in \mathbb{R}^{2} \mid \xi_{1}=\rho_{s w}, 0 \leq \xi_{2} \leq \mu_{s w}\right\}
$$

such that

$$
\xi(0) \in \Omega_{0} \Longrightarrow \xi_{1}(t) \geq \rho_{s w}, \quad t \geq 0
$$




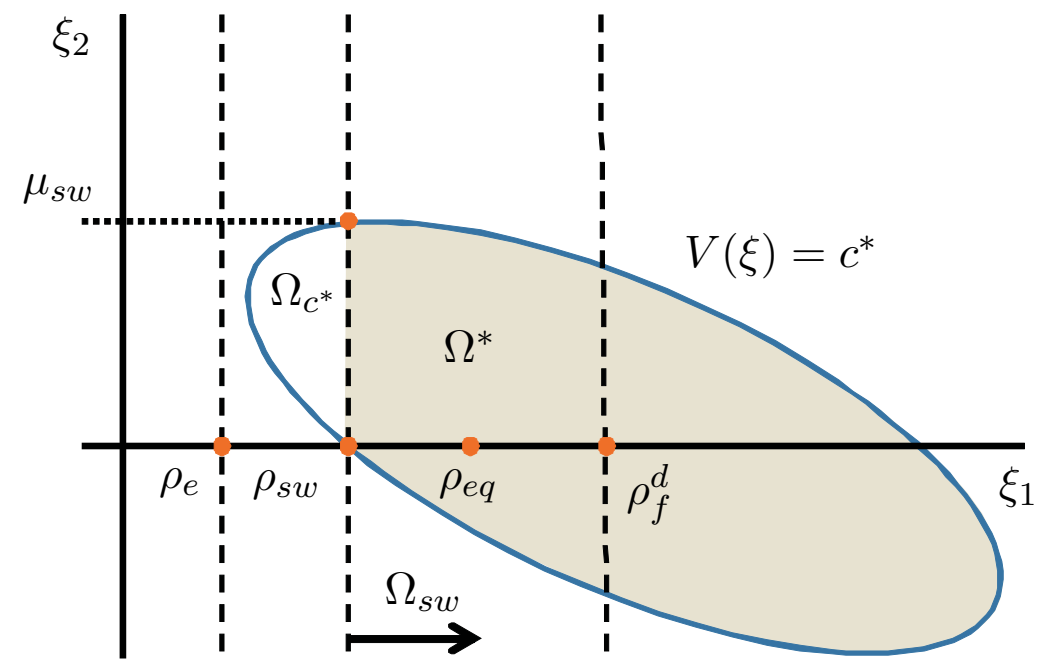

Figure 5.4: The level set $V(\xi)=c^{*}$ and invariant set $\Omega^{*}$ of Lemma 5.2

Proof. Let $Q$ be positive definite. In light of Assumption 5.1, there exists $P$ symmetric, positive definite such that $A^{T} P+P A=-Q$. We choose the Lyapunov function $V(\xi)=\left(\xi-\xi_{f}^{d}\right)^{T} P(\xi-$ $\left.\xi_{f}^{d}\right)$, where $\xi_{f}^{d}=\left(\rho_{f}^{d}, 0\right)$. For $c>0$, define the sublevel set $\Omega_{c}:=\left\{\xi \in \mathbb{R}^{2} \mid V(\xi) \leq c\right\}$. By direct calculation we can verify that (4.43e) is equivalent to $\dot{\xi}=A\left(\xi-\xi_{f}^{d}\right)$ where $A$ is given by (5.2). Then one can verify that $\dot{V}=-\left(\xi-\xi_{f}^{d}\right)^{T} Q\left(\xi-\xi_{f}^{d}\right)<0$. We conclude that $\Omega_{c}$ is positively invariant. Now define the sets

$$
\begin{aligned}
\Omega_{s w} & :=\left\{\xi \in \mathbb{R}^{2} \mid \xi_{1} \geq \rho_{s w}\right\} \\
\Omega^{*} & :=\Omega_{c^{*}} \cap \Omega_{s w},
\end{aligned}
$$

where $c^{*}:=V\left(\left(\rho_{s w}-\rho_{f}^{d}, 0\right)\right)$. See Figure 5.4. We claim that $\Omega^{*}$ is positively invariant.

First, observe that $c^{*}$ is selected so that the point $\left(\rho_{s w}, 0\right)$ lies in $\partial \Omega^{*}$. Now we claim that the set $\partial \Omega_{s w} \cap \Omega^{*}$ is a segment from $\left(\rho_{s w}, 0\right)$ to $\left(\rho_{s w}, \mu_{s w}\right)$ where $\mu_{s w}>0$. To that end, suppose $V(\xi)=\left(\xi-\xi_{f}^{d}\right)^{T} P\left(\xi-\xi_{f}^{d}\right)$ is parametrized by parameters $\left(\alpha_{1}, \alpha_{2}, \alpha_{3}\right)$ as $V(\xi)=$ $\alpha_{1}\left(\xi_{1}-\rho_{f}^{d}\right)^{2}+\alpha_{2}\left(\xi_{1}-\rho_{f}^{d}\right) \xi_{2}+\alpha_{3} \xi_{2}^{2}$. Note that because $P$ is positive definite, we have that $\alpha_{i}>0, i=1,2,3$. Then by equating the parametrized formula for $V$ at points $\left(\rho_{s w}, 0\right)$ and $\left(\rho_{s w}, \mu_{s w}\right)$ we obtain the formula for $\mu_{s w}$ :

$$
\mu_{s w}=\frac{\alpha_{2}}{\alpha_{3}}\left(\rho_{f}^{d}-\rho_{s w}\right)
$$


We observe that $\mu_{s w}>0$ so long as $\rho_{f}^{d}>\rho_{s w}$, as desired. Returning to the claim that $\Omega^{*}$ is positively invariant, we have that $\dot{\xi}_{1}=\xi_{2} \geq 0$ for all $\xi \in \partial \Omega_{s w} \cap \Omega^{*}$, since $\xi_{2} \geq 0$. Combining with the positive invariance of $\Omega_{c^{*}}$, we get $\Omega^{*}$ is positively invariant.

The overall result now follows because $\Omega_{0} \subset \Omega^{*}, \Omega^{*}$ is positively invariant, and by definition of $\Omega^{*}, \xi_{1} \geq \rho_{s w}$ for all $\xi \in \Omega^{*}$.

We remark that under the stated assumptions, the set of initial conditions $\Omega_{0}$ given above is a conservative computation of those that guarantee entrance to the invariant set $\Omega^{*}$. For example, for system (4.43e) there may exist initial conditions $\xi_{0} \in \tilde{\Omega}_{0}:=\left\{\xi \in \mathbb{R}^{2} \mid \xi_{1}=\right.$ $\left.\rho_{s w}, \xi_{2}>\mu_{s w}\right\}$ for which trajectories evolve and eventually enter $\Omega^{*}$ at a time $\tilde{t}>0$ while satisfying $\xi_{1}>\rho_{s w}, t \in(0, \tilde{t}]$. The conservative nature of the result stems from the derivation of an invariant set that depends on a sub-level set of a Lyapunov function.

Equation (5.13) gives the relationship between the switching velocity $\mu_{s w}$ and the matrix $P$ of the Lyapunov function. In particular, given $\mathrm{P}$, we obtain $\mu_{s w}$ such that the segment delimited by the points $\left(\rho_{s w}, 0\right)$ and $\left(\rho_{s w}, \mu_{s w}\right)$ form part of the boundary of an invariant set which is used to prevent a return to reach control from force control. Now we seek the opposite implication. Namely, that a desired maximum switching velocity $\mu_{s w}^{d}$ is selected by the designer corresponding to the maximum allowed velocity of impact for safety (recall this issue is discussed in Section 5.1). Next, $P$ is derived using this $\mu_{s w}^{d}$ to yield the same outcome of a suitable invariant set. However, it is not clear how a given $\mu_{s w}^{d}$ leads to a choice of $P$.

To that end, suppose we are given $A$ from (5.2), $f_{s w}, f_{s}^{d}$, and $Q=\left[\begin{array}{ll}Q_{1} & Q_{2} \\ Q_{2} & Q_{3}\end{array}\right]$ symmetric, positive definite. Let $P=\left[\begin{array}{ll}P_{1} & P_{2} \\ P_{2} & P_{3}\end{array}\right]$. We substitute $A$ given in (5.2) and $Q$ in its parametrized form, and solve for $P$ in the Lyapunov equation $A^{T} P+P A=-Q$. Let $\alpha_{1}:=P_{1}, \alpha_{2}:=2 P_{2}$, $\alpha_{3}:=P_{3}$. Then as in the proof of Lemma $5.2, V(\xi)=\alpha_{1}\left(\xi_{1}-\xi_{f}^{d}\right)^{2}+\alpha_{2}\left(\xi_{1}-\xi_{f}^{d}\right) \xi_{2}+\alpha_{3} \xi_{2}^{2}$, where

$$
\alpha_{2}=\frac{Q_{1} m_{1}}{\bar{k} k_{1}}, \quad \alpha_{3}=\left(\frac{Q_{1} m_{1}}{\bar{k} k_{1}}+Q_{3}\right) \frac{m_{1}}{2 \bar{k} k_{2}} .
$$

Next, we substitute $\alpha_{2}$ and $\alpha_{3}$ into (5.13), and we use (4.43f) and (5.3) to substitute for $\rho_{f}^{d}$ and 
$\rho_{s w}$ respectively to obtain

$$
\mu_{s w}=\frac{\alpha_{2}}{\alpha_{3}}\left(\rho_{f}^{d}-\rho_{s w}\right)=2\left(\left|f_{s}^{d}\right|-\left|f_{s w}\right|\right) \frac{k_{2}}{m_{1}} \frac{Q_{1}}{Q_{1}+\frac{\bar{k} k_{1}}{m_{1}} Q_{3}}
$$

Now observe that $0<\frac{Q_{1}}{Q_{1}+\frac{\bar{k} k_{1}}{m_{1}} Q_{3}}<1$, and that $\lim _{Q_{1} \rightarrow \infty} \frac{Q_{1}}{Q_{1}+\frac{\bar{k} k_{1}}{m_{1}} Q_{3}}=1$. Based on this observation we define the velocity $\bar{\mu}_{s w}$ by

$$
\bar{\mu}_{s w}:=\lim _{Q_{1} \rightarrow \infty} \mu_{s w}=2\left(\left|f_{s}^{d}\right|-\left|f_{s w}\right|\right) \frac{k_{2}}{m_{1}}
$$

Clearly $\bar{\mu}_{s w}$ is the least upper bound of $\mu_{s w}$; that is, for every $\bar{k} \in\left[\bar{k}_{\text {min }}, \bar{k}_{\text {max }}\right], \mu_{s w} \in\left(0, \bar{\mu}_{s w}\right)$. Notice that the parameters of $\bar{\mu}_{s w}$ are given in Problem 5.1 (except for $f_{s w}$ ), and that they correspond to properties of the force controller and switching logic. Then through this analysis method $\bar{\mu}_{s w}$, as the upper limit of $\mu_{s w}$, constitutes a performance measure of the given constrained motion phase. This inherent system constraint is to be accommodated by the design herein.

In (5.15) we have obtained a relationship between the entries of $Q, \bar{k}$, and $\mu_{s w}$. Using this relationship we adopt the following design methodology. First, select the desired switching velocity $\mu_{s w}^{d} \in\left(0, \bar{\mu}_{s w}\right)$. Then construct the following symmetric, positive definite matrix $Q^{d}$ in terms of parameters that are all known

$$
Q^{d}=\left[\begin{array}{ll}
Q_{1} & Q_{2} \\
Q_{2} & Q_{3}
\end{array}\right], \quad Q_{1}=\sqrt{\frac{\frac{\bar{k}_{\max } k_{1}}{m_{1}} \mu_{s w}^{d}}{\bar{\mu}_{s w}-\mu_{s w}^{d}}}>0, \quad 0 \leq Q_{2}<1, \quad Q_{3}=Q_{1}^{-1}
$$

Observe that the bounds on $\mu_{s w}^{d}$ ensure that $Q_{1} \in(0, \infty)$ and $Q_{3}$ is finite. The formula for $Q_{1}$ in (5.17) is derived by substituting $Q_{3}=Q_{1}^{-1}, \bar{k}=\bar{k}_{k m a x}$, and $\mu_{s w}=\mu_{s w}^{d}$ into (5.15) and solving for $Q_{1}$. Also, $Q_{2}$ is selected to be any value such that $Q^{d}$ is positive definite.

In the next result we show that if $\mu_{s w}^{d}$ is given by the designer and $Q^{d}$ is selected according to $(5.17)$ then when the system $(4.43 \mathrm{c})$ reaches a set of states corresponding to $f_{s}(\xi)=f_{s w}$ and $0 \leq \xi_{2} \leq \mu_{s w}^{d}$, then an invariant set is reached as described in Lemma 5.2. Moreover, this set of states is valid for all values of $\bar{k} \in\left[\bar{k}_{\text {min }}, \bar{k}_{\text {max }}\right]$. Below we use the notation $\rho_{s w}(\bar{k})=\rho_{e}+\frac{\left|f_{s w}\right|}{\bar{k}}$ to explicitly denote the dependence of $\rho_{s w}$ on $\bar{k}$; analogously for $\Omega_{0}(\bar{k})$. 
Lemma 5.3. We are given (4.43e), $f_{s}^{d}$ and $f_{\text {sw }}$ with $\left|f_{s}^{d}\right|>\left|f_{\text {sw }}\right|, \rho_{e} \in\left[\rho_{\text {emin }}, \rho_{\text {emax }}\right], \bar{k} \in$ $\left[\bar{k}_{\text {min }}, \bar{k}_{\text {max }}\right]$, and $\bar{\mu}_{s w}$ from (5.16). Suppose that $\mu_{s w}^{d} \in\left(0, \bar{\mu}_{s w}\right)$, and that $Q^{d}$ positive definite is given by (5.17). Then for all $\bar{k} \in\left[\bar{k}_{\text {min }}, \bar{k}_{\text {max }}\right]$ there exists a set of initial conditions

$$
\Omega_{0}^{d}(\bar{k}):=\left\{\xi \in \mathbb{R}^{2} \mid \xi_{1}=\rho_{s w}(\bar{k}), 0 \leq \xi_{2} \leq \mu_{s w}^{d}\right\}
$$

where $\rho_{s w}(\bar{k})=\rho_{e}+\frac{\left|f_{s w}\right|}{\bar{k}}$ such that $\xi(0) \in \Omega_{0}^{d}$ implies $\xi_{1}(t) \geq \rho_{s w}(\bar{k}), t \geq 0$.

Proof. Let $\bar{k} \in\left[\bar{k}_{\text {min }}, \bar{k}_{\text {max }}\right]$ be arbitrary. Using (4.43f), define $\rho_{f}^{d}:=\rho_{e}+\frac{\left|f_{s}^{d}\right|}{\bar{k}}$. Clearly since $\left|f_{s w}\right|<\left|f_{s}^{d}\right|$ we have $\rho_{e}<\rho_{s w}(\bar{k})<\rho_{f}^{d}$. Now applying Lemma 5.2 with $Q=Q^{d}$ we obtain $\mu_{s w}>0$ and a set of initial conditions $\Omega_{0}(\bar{k}):=\left\{\xi \in \mathbb{R}^{2} \mid \xi_{1}=\rho_{s w}(\bar{k}), 0 \leq \xi_{2} \leq \mu_{s w}\right\}$, such that if $\xi(0) \in \Omega_{0}(\bar{k})$, then $\xi_{1}(t) \geq \rho_{s w}(\bar{k})$, for all $t \geq 0$. Now we claim that $\mu_{s w} \in\left[\mu_{s w}^{d}, \bar{\mu}_{s w}\right)$. Proof of claim: following earlier derivations and with $Q_{3}=Q_{1}^{-1}, \mu_{s w}$ can be rewritten as in (5.15) to obtain

$$
\mu_{s w}=2\left(\left|f_{s}^{d}\right|-\left|f_{s w}\right|\right) \frac{k_{2}}{m_{1}} \frac{Q_{1}}{Q_{1}+\frac{\bar{k} k_{1}}{m_{1}} Q_{3}}=\bar{\mu}_{s w} \frac{Q_{1}^{2}}{Q_{1}^{2}+\frac{\bar{k} k_{1}}{m_{1}}} .
$$

Combining (5.19) and (5.17), and $\bar{k}_{\max } \geq \bar{k}$, we have $\mu_{s w}=\bar{\mu}_{s w} \frac{Q_{1}^{2}}{Q_{1}^{2}+\frac{\bar{k} k_{1}}{m_{1}}} \geq \bar{\mu}_{s w} \frac{Q_{1}^{2}}{Q_{1}^{2}+\frac{\bar{k}_{\max k_{1}}}{m_{1}}}=\mu_{s w}^{d}$. Moreover, since $0<\frac{Q_{1}^{2}}{Q_{1}^{2}+\frac{\bar{k} k_{1}}{m_{1}}}<1$ then $\mu_{s w}<\bar{\mu}_{s w}$. This proves the claim. Finally, we show that $\Omega_{0}^{d}(\bar{k}) \subset \Omega_{0}(\bar{k})$. But this follows immediately from the fact that $\left[0, \mu_{s w}^{d}\right] \subset\left[0, \mu_{s w}\right]$. Therefore by Lemma $5.2, \xi(0) \in \Omega_{0}^{d}(\bar{k})$ implies $\xi(0) \in \Omega_{0}(\bar{k})$ implies $\xi_{1}(t) \geq \rho_{s w}(\bar{k})$ for all $t \geq 0$. Equivalently, (5.18) holds.

The previous three lemmas show that all states of the closed-loop system will arrive at the switching boundary $\Gamma_{s w}$ in $\mathcal{P}^{3}$ corresponding to velocities in the range $0 \leq \xi_{2} \leq \mu_{s w}$. Since the force dynamics are stabilized by the force feedback (4.42) the sensed force will converge to the desired force without a switch back into reach control. Therefore, we have obtained a specific relationship between the desired force, switching force, and system velocity such that a bounce does not occur.

The following algorithm summarizes the steps to select the problem parameters enroute to completing the definition of the logic control specifications. 
Algorithm 5.1. We consider the model (4.43a)-(4.43b) along with a force controller (4.42). We are given the system parameters masses $m_{1}$ and $m_{2}$ with $m_{1}>>m_{2}$; sensed force parameters $k_{s}$ and $r_{s}$; the range for the environment spring constant, $k_{e} \in\left[k_{e m i n}, k_{e m a x}\right]$; the range for the effective spring constant, $\bar{k} \in\left[\bar{k}_{\text {min }}, \bar{k}_{\text {max }}\right]$; the range of the environment position $\hat{\rho}_{e} \in$ $\left[\hat{\rho}_{\text {emin }}, \hat{\rho}_{\text {emax }}\right]$ where $0 \leq \hat{\rho}_{\text {emin }} \leq \hat{\rho}_{\text {emax }}$; and force controller parameters $k_{1}, k_{2}$, and $f_{s}^{d}$. Finally, we are given the specification parameters $\rho_{0}$ and $\mu_{1}$.

1. Select a switching force threshold $f_{s w}$, where $\left|f_{s w}\right|<\left|f_{s}^{d}\right|$.

2. Compute $\bar{\mu}_{s w}=2\left(\left|f_{s}^{d}\right|-\left|f_{s w}\right|\right) \frac{k_{2}}{m_{1}}$. Choose $\mu_{s w}$ such that $0<\mu_{s w}<\bar{\mu}_{s w}$.

3. Choose $\rho_{2}$ according to $\rho_{2}>\rho_{\text {emax }}+\frac{\left|f_{s w}\right|}{\bar{k}_{\min }}$.

4. Select the velocity parameter $\mu_{2}$ such that $\mu_{s w}<\mu_{2}<\mu_{1}$. Compute the position $\rho_{1}=$ $\rho_{\text {emin }}+\left(\rho_{2}-\rho_{\text {emin }}\right) \frac{\mu_{s w}-\mu_{2}}{\mu_{s w}}$ so that $\mathcal{P}^{3}$ is a simplex with vertices $v_{0}=\left(\rho_{1}, \mu_{2}\right), v_{1}=\left(\rho_{1}, 0\right)$, $v_{2}=\left(\rho_{2}, 0\right)$.

5. Select the position $\rho_{3}$ such that $\rho_{0}<\rho_{3}<\rho_{1}$.

The algorithm provides all vertices of the polytope $\mathcal{P}$. This polytope inherently constrains the states and trajectories of the manipulator to those that satisfy the impact velocity and bumpless transfer requirements. Next we partition $\mathcal{P}$ into five polytopes $\mathcal{P}^{i}, i=1, \ldots, 5$. Reach controllers $u_{r c p}^{i}(\xi), i=1, \ldots, 5$ are specified on each polytope $\mathcal{P}^{i}$ so that $\mathcal{P}^{1} \stackrel{\mathcal{P}^{1}}{\longrightarrow} \mathcal{F}_{0}$, $\mathcal{P}^{2} \stackrel{\mathcal{P}^{2}}{\longrightarrow} \mathcal{F}_{1}, \mathcal{P}^{4} \stackrel{\mathcal{P}^{4}}{\longrightarrow} \mathcal{F}_{3}$, and $\mathcal{P}^{5} \stackrel{\mathcal{P}^{5}}{\longrightarrow} \mathcal{F}_{2}$. For $\mathcal{P}^{3}, u_{r c p}^{3}$ will be designed according to Lemma 5.1. Collectively, all RCP controllers $u_{r c p}^{i}$ will be denoted by $u_{r c p}$. The overall switching controller is given as follows:

$$
u\left(\xi, f_{s}\right)=\left\{\begin{array}{cc}
u_{r c p}^{i}(\xi), & \xi \in \mathcal{P}^{i}, i \in\{1,2,4,5\} \\
u_{r c p}^{3}(\xi), & \xi \in \mathcal{P}^{3} \text { and }\left|f_{s}\right|<\left|f_{s w}\right| \\
u_{f}, & \left|f_{s}\right| \geq\left|f_{s w}\right| .
\end{array}\right.
$$

Notice that the switching logic for entering force control need only consider the force threshold $f_{s w}$. The requirement that $0 \leq \xi_{2} \leq \mu_{s w}$ is inherently met by the structure of $\mathcal{P}^{3}$. The following is the main result of this thesis. 
Theorem 5.1. Consider the systems (4.43a) - (4.43e) and the polytope $\mathcal{P}$ whose vertices are determined by the Algorithm 5.1. Consider the controller $u(\xi)$ given in (5.20). The closed-loop system satisfies the following:

(i) For all $\xi(0) \in \mathcal{P}^{1}, \xi(t) \rightarrow\left(\rho_{f}^{d}, 0\right)$ as $t \rightarrow \infty$; correspondingly the steady state contact force is $f_{s}^{d}$.

(ii) The controller (5.20) switches from reach control $u_{r c p}$ to force control $u_{f}$ only once.

(iii) Impact with the environment occurs with a velocity bounded by $\mu_{s w}$.

Proof. The proof begins by enumerating all behaviors generated by $u_{r c p}(\xi)$. On $\mathcal{P}^{1}$ the system is (4.43a) and the controller is $u_{r c p}^{1}$. For (4.43a) we have $\mathcal{O}=\left\{\xi \in \mathbb{R}^{2} \mid \xi_{2}=0\right\}$. It is easily verified that there exist control values $u_{j}^{i}$ on the vertices $v_{j}^{i}$ of $\mathcal{P}^{1}$ which satisfy the invariance conditions $(3.5)$ for $\mathcal{P}^{1}$ with exit facet $\mathcal{F}_{0}$. Let $v_{1}^{1}:=\left(\rho_{0}, 0\right)$ and $v_{2}^{1}:=\left(\rho_{3}, 0\right)$. Then we observe that $\mathcal{P}^{1} \cap \mathcal{O}=\operatorname{co}\left\{v_{1}^{1}, v_{2}^{1}\right\}$. Since $B=(0,1)$, we select $u_{1}^{1}>0$ and $u_{2}^{1}>0$ to satisfy the invariance conditions on $v_{1}^{1}$ and $v_{2}^{1}$ respectively. Now we apply Theorem 3.10. Since the invariance conditions hold by design, and clearly $0 \notin \operatorname{co}\left\{B u\left(v_{1}^{1}\right), B u\left(v_{2}^{1}\right)\right\}$, we have that $\mathcal{P}^{1} \stackrel{\mathcal{P}^{1}}{\longrightarrow} \mathcal{F}_{0}$ using $u_{r c p}^{1}(\xi)$. Similar arguments as for $\mathcal{P}^{1}$ show that there exist $u_{r c p}^{i}(\xi), i=2,4,5$ such that $\mathcal{P}^{2} \stackrel{\mathcal{P}^{2}}{\longrightarrow} \mathcal{F}_{1}, \mathcal{P}^{4} \stackrel{\mathcal{P}^{4}}{\longrightarrow} \mathcal{F}_{3}$, and $\mathcal{P}^{5} \stackrel{\mathcal{P}^{5}}{\longrightarrow} \mathcal{F}_{2}$.

On $\mathcal{P}^{3}$, Lemma 5.1 explicitly provides an affine feedback $u_{r c p}^{3}(\xi)$ that guarantees that trajectories of the non-contact system (4.43a) arriving in $\mathcal{P}^{3}$ from $\mathcal{P}^{2}$ reach a state when $\xi_{1} \geq \rho_{\text {emax }}$. Thus, necessarily contact is made with the environment and the model switches to (4.43c). Lemma 5.1 further shows that under $u_{r c p}^{3}$, trajectories of $(4.43 \mathrm{c})$ reach the switching boundary $\Gamma_{s w}$ when $f_{s}=f_{s w}$. According to (5.20), the controller then switches to $u_{f}$ and the model reverts to $(4.43 \mathrm{e})$. Lemma 5.3 shows that trajectories of $(4.43 \mathrm{e})$ remain in a region of the state space when $\left|f_{s}\right| \geq\left|f_{s w}\right|$ and so the controller (5.20) can never switch back to $u_{r c p}$. This proves (ii). Also (i) follows since $u_{f}$ guarantees that $f_{s}$ reaches a steady-state of $f_{s}^{d}$. Finally, the first (and only) impact with the environment occurs at some time $\bar{t}>0$ when $\xi(\bar{t}) \in \mathcal{P}^{3}$ and $\xi_{1}(\bar{t})=\rho_{s w}$. But then by design (see Figures 5.2 and 5.3), $\xi_{2}(\bar{t}) \leq \mu_{s w}$, which proves (iii).

In Theorem 5.1 we obtain the solution to Problem 5.1, which leads to the execution of the interaction task featuring a bumpless transfer. Next we apply the developed theory to a planar manipulator system, and present the design of the reach controllers. 


\section{Chapter 6}

\section{Control Design for Three-Link}

\section{Manipulator}

In this chapter we use the models of Chapter 4 and methodology of Chapter 5 to synthesise reach controllers that solve Problem 5.1 and achieve a bumpless transfer between reach and force control. First the system parameters of an arbitrary manipulator are defined, and then we proceed with the control design process to generate an overall hybrid controller. Finally, a variety of simulations are performed to validate the design.

\subsection{Reach Control Design}

In this section we assume a nominal set of parameters for Problem 5.1, and design a set of reach controllers according to the methods of Chapter 5. The intention here is to define a "larger" manipulator system, which is meant to contrast the relatively smaller Special Purpose Dextrous Manipulator (SPDM), which is the subject of the next two chapters. Specifically, we define first the physical parameters of an arbitrary three-link manipulator and second mass with component values larger than those that appear in the SPDM simulator. Referring back to the system diagram in Figure 4.5, let the link lengths be $l_{1}=6 \mathrm{~m}, l_{2}=6 \mathrm{~m}, l_{3}=3 \mathrm{~m}$, with masses $m_{l 1}=300 \mathrm{~kg}, m_{l 2}=300 \mathrm{~kg}, m_{l 3}=100 \mathrm{~kg}$, and moments of inertia about the $z$-axis $I_{l 1}=903 \mathrm{kgm}^{2}, I_{l 2}=903 \mathrm{kgm}^{2}, I_{l 3}=76 \mathrm{kgm}^{2}$. Moreover, we assume that $m_{2}=50 \mathrm{~kg}$. Now from Problem 5.1 we select nominal system parameters. In accordance with the assumption 
that $m_{1}>>m_{2}$ we set $m_{1}=5000 \mathrm{~kg}$ such that $m_{1} / m_{2}=100$. Note that the sensor stiffness is chosen as $k_{s}=10^{7} \mathrm{~N} / \mathrm{m}$ which is similar to, but different than, the sensor stiffness that appears in the SPDM simulator. The rest length is then chosen arbitrarily as $r_{s}=0.005 \mathrm{~m}$. Next, the environment is defined to be reasonably stiff, where $k_{e} \in\left[10^{6}, 5 \cdot 10^{7}\right] \mathrm{N} / \mathrm{m}$. The effective spring constant range is then $\bar{k} \in\left[9.0909\left(10^{5}\right), 8.3333\left(10^{6}\right)\right] \mathrm{N} / \mathrm{m}$. We assume that the environment position is known within a range of $2 \mathrm{~cm}$ where $\hat{\rho}_{e} \in[12.005,12.025] \mathrm{m}$, similarly $\rho_{e} \in[12.0,12.02] \mathrm{m}$. Moreover we set $\rho_{0}=0 \mathrm{~m}$, and select $\mu_{1}=0.35 \mathrm{~m} / \mathrm{s}$ to limit the maximum manipulator velocity. A force controller must also be defined, which is fixed for the duration of the design. Recall force controller (4.42), and the closed-loop force dynamics (4.43e). Assuming a desired contact force $f_{s}^{d}=-300 \mathrm{~N}$, we design the control parameters $k_{1}=0.06, k_{2}=0.012$ to generate a critically-damped response with a natural frequency $\omega=10 \mathrm{rad} / \mathrm{s}$, when $\bar{k}=\bar{k}_{\text {max }}$. Parameters $k_{1}$ and $k_{2}$ are designed with respect to $\bar{k}_{\text {max }}$ because the maximum stiffness yields the smallest safe switching velocity, as per the development preceding and including Lemma 5.3. Lastly, we design the PD controllers for task space variables $y_{1}$ and $\phi$ to be critically damped with natural frequency $\omega_{y}=2 \mathrm{rad} / \mathrm{s}$ and $\omega_{\phi}=1 \mathrm{rad} / \mathrm{s}$ respectively.

Now for the design we use Algorithm 5.1 to select or calculate the switching force $f_{s w}$, and the remaining parameters $\rho_{i}, \mu_{i}$ defined by the logic control specifications. In open-loop the sensed force dynamics are defined by a stiffness, resulting in an oscillatory measurement. Therefore, the switching force is selected large enough to avoid the case of spurious switching due to natural oscillations (and not due to contact). Conversely, as $\left|f_{s w}\right|$ increases, $\bar{\mu}_{s w}$ decreases, so the magnitude should not be arbitrarily large. Thus we select $f_{s w}=-30 \mathrm{~N}$. Next, we compute $\bar{\mu}_{s w}=2\left(\left|f_{s}^{d}\right|-\left|f_{s w}\right|\right) \frac{k_{2}}{m_{1}}=1.296 \mathrm{~mm} / \mathrm{s}$, and select $\mu_{s w}$ as large as possible while still providing some margin, i.e. $\mu_{s w}=1.2 \mathrm{~mm} / \mathrm{s}$. In Steps $3-4$ we select the remaining parameters that define $\mathcal{P}^{3}$. First we choose $\rho_{2}>\rho_{\text {emax }}+\frac{\left|f_{s w}\right|}{\bar{k}_{\min }}$ to ensure that the end effector remains in $\mathcal{P}^{3}$ prior to the switch to force control. Selecting $\rho_{2}$ sufficiently large provides robustness against additional variation in $\rho_{e}$ and $\bar{k}$, i.e. $\rho_{2}=12.27 \mathrm{~m}>12.02+\frac{30}{\bar{k}_{\text {min }}}$. Note that $\rho_{2}$ is also selected to avoid a manipulator singularity, in this case when the arm is fully extended and $\theta_{2}=0^{\circ}$. Assuming $y_{1}^{d}=0 \mathrm{~m}$, this requires $\rho_{2}<l_{1}+l_{2}+l_{3}=15 \mathrm{~m}$. Next we select $\mu_{2}=1.3 \mathrm{~mm} / \mathrm{s}>\mu_{s w}$, which is approximately equal to $\bar{\mu}_{s w}$; in this manner every possible velocity in $\mathcal{P}^{3}$ is considered safe for achieving bumpless transfer. Next we compute $\rho_{1}=\rho_{\text {emin }}+\left(\rho_{2}-\rho_{\text {emin }}\right) \frac{\mu_{s w}-\mu_{2}}{\mu_{s w}}=11.9775 \mathrm{~m}$. 


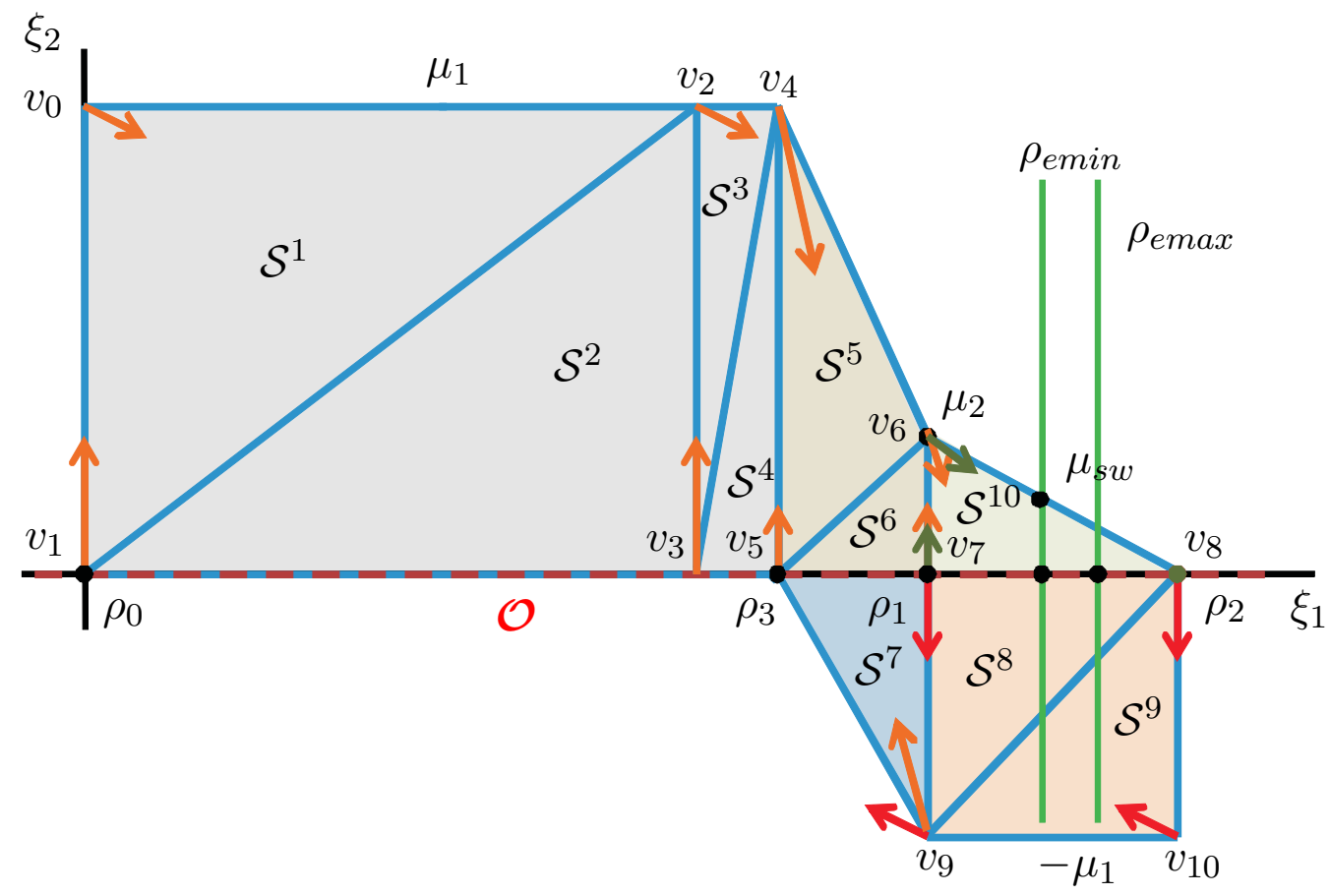

Figure 6.1: The triangulated state space with desired closed-loop velocity vectors at the vertices.

Note that if the computed value of $\rho_{1}$ is undesirable, say $\rho_{1}$ is considered to be too close to $\rho_{\text {emin }}$, then Steps 3-4 may be repeated. For example, assuming the original selection of $\mu_{2}$ is retained, then increasing $\rho_{2}$ will decrease $\rho_{1}$. Finally we choose $\rho_{3}=11.5 \mathrm{~m}<\rho_{1}$ to provide sufficient space for the manipulator to begin deceleration. This set of nominal parameters is compiled into Table 6.1 for quick reference. With the $\rho_{i}$ and $\mu_{i}$ in hand the polytope $\mathcal{P}$ is numerically defined. Next subdivide $\mathcal{P}$ into the regions $\mathcal{P}^{j}, j=1, \ldots, 5$ (see Figure 5.2), in which the states $\xi$ are constrained as follows

$$
\begin{array}{ccc}
\mathcal{P}^{1}: & 0 \leq \xi_{1} \leq 11.5 & 0 \leq \xi_{2} \leq 0.35 \\
\mathcal{P}^{2}: & 11.5 \leq \xi_{1} \leq 11.9775 & 0 \leq \xi_{2} \leq 8.7481-0.7303 \xi_{1} \\
\mathcal{P}^{3}: & 11.9775 \leq \xi_{1} \leq 12.27 & 0 \leq \xi_{2} \leq 0.0044\left(12.27-\xi_{1}\right) \\
\mathcal{P}^{4}: & 11.9775 \leq \xi_{1} \leq 12.27 & -0.35 \leq \xi_{2} \leq 0 \\
\mathcal{P}^{5}: & 11.5 \leq \xi_{1} \leq 11.9775 & -0.733\left(\xi_{1}-11.5\right) \leq \xi_{2} \leq 0
\end{array}
$$

Now we perform the reach control design using (4.43a) for no contact. First we calculate the set of possible equilibria $\mathcal{O}=\left\{\xi \mid \xi_{2}=0\right\}$. Then $\mathcal{P}$ is divided based on the triangulation $\mathcal{T}=\left\{\mathcal{S}^{1}, \ldots, \mathcal{S}^{10}\right\}$ consisting of ten $2 \mathrm{D}$ simplices as shown in Figure 6.1; note that many possible subdivisions exist that will give similar acceptable behaviour. By this triangulation we have 
split $\mathcal{P}^{1}$ into two sub-polytopes, denoted $\mathcal{P}_{1}^{1}$ and $\mathcal{P}_{2}^{1}$, where $\mathcal{P}_{1}^{1}:=\mathcal{S}^{1} \cup \mathcal{S}^{2}$ and $\mathcal{P}_{2}^{1}:=\mathcal{S}^{3} \cup \mathcal{S}^{4}$. We additionally comment that the intersection of each simplex $\mathcal{S}^{i}$ with the set $\mathcal{O}$ occurs along a face of each simplex. This selection of triangulation makes possible the use of various results in the literature for such triangulations [33].

Next, we label the vertices of $\mathcal{P}: v_{0}=(0,0.35), v_{1}=(0,0), v_{2}=(11.4,0.35), v_{3}=(11.4,0)$ $v_{4}=(11.5,0.35), v_{5}=(11.5,0), v_{6}=(11.9775,0.0013), v_{7}=(11.9775,0), v_{8}=(12.27,0)$, $v_{9}=(11.9775,-0.35)$, and $v_{10}=(12.27,-0.35)$. From the temporal sequence of Problem 5.1, red arrows in Figure 5.2 mark exit facets of each $\mathcal{P}^{j}$ in order to drive trajectories into $\mathcal{P}^{3}$ (in which an equilibrium is created at $v_{8}$ ). To that end the arrows in Figure 6.1 depict desired closed-loop velocities to be achieved by appropriate controls at the vertices (note, the regions defining the $\mathcal{P}^{j}$ are shaded different colours). Some vertices have multiple vectors, each requiring a distinct control value to generate a distinct continuous affine controller. The vectors for each $\mathcal{P}^{j}$ are differentiated by colour: orange for $\mathcal{P}^{1}, \mathcal{P}^{2}$, and $\mathcal{P}^{5}$, green for $\mathcal{P}^{3}$, and red for $\mathcal{P}^{4}$. This arrangement produces a piecewise affine feedback that is continuous for transitions between $\mathcal{P}^{1}, \mathcal{P}^{2}$, and $\mathcal{P}^{5}$, and discontinuous for all other transitions.

We now walk through the control selection for each region. Let $u_{i}^{j} \in \mathbb{R}^{m}$ denote the control value at vertex $v_{i}, i=1, \ldots, 10$, and in region $\mathcal{P}^{j}, j \in\{1,2,3,4,5\}$, such that in Figure 6.1 vectors $A v_{i}+B u_{i}^{j}+a$ are attached at each vertex. Observe that the vertices $v_{k}, k=1,3,5,7,8$, lie on the set $\mathcal{O}$ such that equilibria may be generated in any $\mathcal{P}^{j}$. The only desirable equilibrium

Table 6.1: Parameters of Problem 5.1 and Algorithm 5.1.

\begin{tabular}{llll}
\multicolumn{3}{c}{ Problem 5.1 Parameters } & \\
\hline \hline$m_{1}$ & $5000 \mathrm{~kg}$ & $r_{s}$ & $0.005 \mathrm{~m}$ \\
$m_{2}$ & $50 \mathrm{~kg}$ & $k_{1}$ & 0.06 \\
$\rho_{e}$ & {$[12,12.02] \mathrm{m}$} & $k_{2}$ & 0.012 \\
$k_{e}$ & {$\left[10^{6}, 5 \cdot 10^{7}\right] \mathrm{N} / \mathrm{m}$} & $f_{s}^{d}$ & $-300 \mathrm{~N}$ \\
$k_{s}$ & $10^{7} \mathrm{~N} / \mathrm{m}$ & $\rho_{0}$ & $0 \mathrm{~m}$ \\
$\bar{k}$ & {$\left[9.0909\left(10^{5}\right), 8.3333\left(10^{6}\right)\right] \mathrm{N} / \mathrm{m}$} & $\mu_{1}$ & $0.35 \mathrm{~m} / \mathrm{s}$ \\
$k_{p y}$ & 4 & $k_{p \phi}$ & 1 \\
$k_{d y}$ & 4 & $k_{d \phi}$ & 2
\end{tabular}

Algorithm 5.1 Parameters

\begin{tabular}{llllll}
\hline \hline$f_{s w}$ & $-30 \mathrm{~N}$ & $\mu_{s w}$ & $1.2 \mathrm{~mm} / \mathrm{s}$ & $\rho_{1}$ & $11.9775 \mathrm{~m}$ \\
$\bar{\mu}_{s w}$ & $1.296 \mathrm{~mm} / \mathrm{s}$ & $\mu_{2}$ & $1.3 \mathrm{~mm} / \mathrm{s}$ & $\rho_{2}$ & $12.27 \mathrm{~m}$ \\
& & & & $\rho_{3}$ & $11.5 \mathrm{~m}$
\end{tabular}


is to be at $v_{8}$ of $\mathcal{P}^{3}$; to avoid generating additional equilibria the control values at the $v_{k}$ must satisfy $A v_{k}+B u_{k}^{j}+a \neq 0, k \neq 8, j \neq 3$. Following the methods of Chapter 3 (see in particular Example 3.4), we select control values $u_{i}^{j}$ so that the invariance conditions are met at each vertex of each $\mathcal{P}^{j}$; conditions (3.16) must be satisfied for polytopes $\mathcal{P}_{1}^{1}, \mathcal{P}_{2}^{1}, \mathcal{P}^{2}, \mathcal{P}^{4}$, while (3.4) must be satisfied for simplices $\mathcal{P}^{3}, \mathcal{P}^{5}$.

In the polytope $\mathcal{P}_{1}^{1}$ the intended behaviour is quick motion in free space; such behaviour may be achieved by setting the magnitude of the controls at $v_{1}, u_{1}^{1}$, and $v_{3}, u_{3}^{1}$, to be larger than those at $v_{0}, u_{0}^{1}$, and $v_{2}, u_{2}^{1}$. Additionally, the invariance conditions are trivially satisfied by setting $u_{1}^{1}$ and $u_{3}^{1}$ positive, and setting $u_{0}^{1}$ and $u_{2}^{1}$ negative:

$$
u_{0}^{1}=-250, \quad u_{1}^{1}=1000, \quad u_{2}^{1}=-250, \quad u_{3}^{1}=1000 .
$$

The purpose of $\mathcal{P}_{2}^{1}$ is to ensure a continuous transition between the quick-motion reach controller of $\mathcal{P}_{1}^{1}$, and the decelerating reach controller to be designed for $\mathcal{P}^{2}$. Consequently, the controls at $v_{2}$ and $v_{3}$ remain the same, while $u_{5}^{1}$ is simply set positive, and $u_{4}^{1}$ is chosen to restrict the facet defined by the segment $\left[v_{4}, v_{6}\right]$ :

$$
u_{4}^{1}=-1400, \quad u_{5}^{1}=540
$$

Region $\mathcal{P}^{2}$ consists of simplices $\mathcal{S}^{5}$ and $\mathcal{S}^{6}$, for which two reach controllers will be synthesized. It will be shown via simulation that undesired spurious switching can sometimes occur amongst these simplices, due to small wavering of the first mass in response to the spring force. Now by designing the two reach controllers to be identical, the spurious switching becomes irrelevant. The strategy for doing so is to define a 'virtual' simplex in which $\mathcal{P}^{2}$ is contained. We then choose the controls of the virtual simplex to obtain a specific behaviour, and then generate a single reach controller for the simplex. Since $\mathcal{P}^{2}$ is contained in the simplex, then the single reach controller is applicable for all $\xi \in \mathcal{P}^{2}$. To that end, extend the line segments $\left[v_{4}, v_{6}\right]$ and $\left[v_{5}, v_{7}\right]$ to the point at which they intersect along the $\xi_{1}$ axis, which we denote $v^{\prime}=\left(\rho^{\prime}, 0\right)$. Now consider the virtual simplex $\mathcal{S}^{\prime}=\operatorname{co}\left\{v_{4}, v_{5}, v^{\prime}\right\}$, pictured in Figure 6.2 where clearly $\mathcal{P}^{2} \subset \mathcal{S}^{\prime}$. In a manner analogous to the design of $\mathcal{P}^{3}$ (see Lemma 5.1 part (i)), we design $\mathcal{S}^{\prime}$ to be invariant, and such that trajectories approach the equilibrium that is to be placed at $v^{\prime}$. To place the 


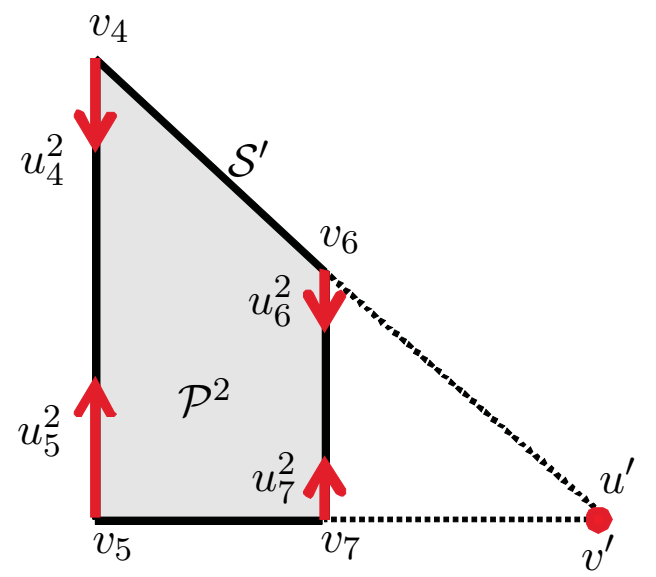

Figure 6.2: Simplex $\mathcal{S}^{\prime}$ used in the design of the reach controller for $\mathcal{P}^{2}$.

equilibrium we set at $v^{\prime}$ the control $u^{\prime}=0$. For $v_{4}$ and $v_{5}$ we observe that by setting $u_{4}^{2}=u_{4}^{1}$, and $u_{5}^{2}=u_{5}^{1}$, the remaining invariance conditions on $\mathcal{S}^{\prime}$ are achieved, and a continuous transition from the reach controller of $\mathcal{P}_{2}^{1}$ is obtained. One can then synthesis a reach controller using the vertices and control values for $\mathcal{S}^{\prime}$. For initial conditions in $\mathcal{P}^{2}$, trajectories enroute to the virtual $v^{\prime}$ will reach the boundary shared by $\mathcal{P}^{2}$ and $\mathcal{P}^{3}$, prompting a transition to the reach controller of $\mathcal{P}^{3}$. Alternatively, we may derive directly the equivalent controls at $v_{6}$ and $v_{7}$ of $\mathcal{P}^{2}$ that may be used to synthesis identical reach controllers for $\mathcal{S}^{5}$ and $\mathcal{S}^{6}$. By convexity we observe that

$$
v_{6}=\frac{\mu_{2}}{\mu_{1}} v_{4}+\left(1-\frac{\mu_{2}}{\mu_{1}}\right) v^{\prime}, \quad v_{7}=\frac{\mu_{2}}{\mu_{1}} v_{5}+\left(1-\frac{\mu_{2}}{\mu_{1}}\right) v^{\prime} .
$$

Therefore, assuming $u_{4}^{2}$ and $u_{5}^{2}$ are already selected and that $u^{\prime}=0$, we choose the controls at the vertices according to the same convex combination

$$
u_{6}^{2}=\frac{\mu_{2}}{\mu_{1}} u_{4}^{2}+\left(1-\frac{\mu_{2}}{\mu_{1}}\right) u^{\prime}=\frac{\mu_{2}}{\mu_{1}} u_{4}^{2}=-5.2, \quad u_{7}^{2}=\frac{\mu_{2}}{\mu_{1}} u_{5}^{2}+\left(1-\frac{\mu_{2}}{\mu_{1}}\right) u^{\prime}=\frac{\mu_{2}}{\mu_{1}} u_{5}^{2}=2.0057 .
$$

With the design of $\mathcal{P}^{2}$ complete we proceed with the simplex $\mathcal{P}^{5}$. A continuous transition is desired between $\mathcal{P}^{5}$ and $\mathcal{P}^{2}$, therefore the control values at vertices $v_{5}$ and $v_{7}$ are chosen as above. The final control at $v_{9}$ is selected to restrict the facet defined by the segment $\left[v_{5}, v_{9}\right]$ :

$$
u_{5}^{5}=u_{5}^{2}=540, \quad u_{7}^{5}=u_{7}^{2}=2.0057, \quad u_{9}^{5}=1500 .
$$


In $\mathcal{P}^{3}$, a simplex, the control selection is more nuanced as two closed-loop behaviours must be achieved. In particular, the control at $v_{8}$ is set to zero to render it an equilibrium. Secondly, contact with the obstacle is made in this region, therefore to ensure that the reach controller drives trajectories to the boundary $\Gamma_{s w}$ the control at $v_{7}$ must satisfy (5.6) of Lemma 5.1

$$
u_{7}^{3}>\frac{\left|f_{s w}\right|\left(\rho_{2}-\rho_{1}\right)}{\rho_{2}-\rho_{e \max }-\frac{\left|f_{s w}\right|}{\bar{k}_{\min }}}=35.1
$$

The remaining control at $v_{6}$ is selected to restrict the facet defined by the segment $\left[v_{6}, v_{8}\right]$ :

$$
u_{6}^{3}=-23.4, \quad u_{7}^{3}=70.2, \quad u_{8}^{3}=0
$$

Lastly, in the polytope $\mathcal{P}^{4}$ the opposite concept of $\mathcal{P}_{1}^{1}$ is applied with the signs reversed. Note that the control at $v_{8}$ is no longer zero as an equilibrium is not intended for trajectories in this region. Instead the controls are selected as

$$
u_{7}^{4}=-500, \quad u_{9}^{4}=2000, \quad u_{8}^{4}=-500, \quad u_{10}^{4}=2000 .
$$

The last step of the control design is to generate the reach controllers for each simplex of the form $u_{r c p}^{i}(\xi)=K^{i} \xi+g^{i}, i=1, \ldots, 10$. For each simplex we consider the vertices and their control values and apply (3.10) to obtain the following reach controllers

$$
\begin{aligned}
& u_{r c p}^{1}=[0-3571] \xi+1000, \quad \xi \in \mathcal{S}^{1} \\
& u_{r c p}^{2}=\left[\begin{array}{ll}
0 & -3571
\end{array}\right] \xi+1000, \quad \xi \in \mathcal{S}^{2} \\
& u_{r c p}^{3}=[-11500-3571] \xi+132100, \quad \xi \in \mathcal{S}^{3} \\
& u_{r c p}^{4}=[-4600-5543] \xi+53440, \quad \xi \in \mathcal{S}^{4} \\
& u_{r c p}^{5}=[-1123-5543] \xi+13497, \quad \xi \in \mathcal{S}^{5} \\
& u_{r c p}^{6}=[-1123-5543] \xi+13497, \quad \xi \in \mathcal{S}^{6} \\
& u_{r c p}^{7}=[-1123-4280] \xi+13497, \quad \xi \in \mathcal{S}^{7} \\
& u_{r c p}^{8}=[0-7143] \xi-500, \quad \xi \in \mathcal{S}^{8} \\
& u_{r c p}^{9}=\left[\begin{array}{ll}
0 & -7143
\end{array}\right] \xi-500 \quad \xi \in \mathcal{S}^{9} \\
& u_{r c p}^{10}=\left[\begin{array}{ll}
-240-72009
\end{array}\right] \xi+2945 \quad \xi \in \mathcal{S}^{10}
\end{aligned}
$$


The set of reach controllers constitutes a piecewise continuous affine feedback, denoted simply as $u_{r c p}$. When the current states of $\left(\xi_{1}, \xi_{2}\right)$ are shared among multiple simplices from different $\mathcal{P}^{j}$ a switch to the next reach controller occurs by the following hierarchy:

$$
\mathcal{P}^{3}>\mathcal{P}^{2}>\mathcal{P}^{1}>\mathcal{P}^{5}>\mathcal{P}^{4}
$$

For example, the point $\left(\rho_{2}, 0\right)$ is shared among the $\mathcal{P}^{j}, j \neq 1$, and would enable the reach controller $u_{r c p}^{10}=K^{10} \xi+g^{10}$ for $\mathcal{S}^{10} \subset \mathcal{P}^{3}$.

Finally the overall hybrid controller is constructed from the reach controllers $u_{r c p}^{i}(\xi)$ for each simplex, and force controller $u_{f}$ given by (4.42). In words, the sensed force $f_{s}$ is compared to the switching force $f_{s w}$ once the first mass reaches $\mathcal{P}^{3}$, equivalently when $\xi_{1} \geq \rho_{1}$; a switch to force control is made if the threshold is exceeded. When the first mass is to the left of $\rho_{1}$ contact is not possible, therefore the reach controllers by default guide the system through the defined state space.

$$
u\left(\xi, f_{s}\right):= \begin{cases}u_{f} & \xi_{1} \geq \rho_{1},\left|f_{s}\right| \geq\left|f_{s w}\right| \\ u_{r c p}^{10}(\xi) & \xi \in \mathcal{S}^{10},\left|f_{s}\right|<\left|f_{s w}\right| \\ u_{r c p}^{i}(\xi) & \xi \in \mathcal{S}^{i}, i \in\{1, \ldots, 9\}\end{cases}
$$

Figures 6.3-6.4 depict the closed-loop phase portraits, in blue, of the second-order dynamics (4.43a) under the reach controllers. The vertical magenta line represents a typical example of the environment location, in this case $\rho_{e}=12.01 \mathrm{~m}$, which corresponds to the true location $\hat{\rho}_{e}=12.015 \mathrm{~m}$. Figure 6.3 depicts the dominate features of $\mathcal{P}$, showing only a portion of $\mathcal{P}_{1}^{1}$. In particular, trajectories approach from the left at high velocity, but well within the upper limit of $\mu_{1}=0.35 \mathrm{~m} / \mathrm{s}$. The thin rectangular region represents $\mathcal{P}_{2}^{1}$, in which the deceleration process begins. These trajectories lead to $\mathcal{P}^{2}$, where the deceleration is more prominent. Moreover in $\mathcal{P}^{5}$ and $\mathcal{P}^{4}$ the vector field clearly indicates that trajectories will move backwards no further than $\xi_{1}=11.5 \mathrm{~m}$, and into $\mathcal{P}^{2}$. From $\mathcal{P}^{2}$ trajectories are steered into $\mathcal{P}^{3}$, which is too small to observe here. Instead, Figure 6.4 depicts $\mathcal{P}^{3}$, wherein trajectories are driven towards the equilibrium at $\left(\rho_{2}, 0\right)$ along a diagonal phase line. This phase line intersects with the dashed line at a velocity of $0.9 \mathrm{~mm} / \mathrm{s}$. As this velocity is less than the selected switching velocity $\mu_{s w}=1.2 \mathrm{~mm} / \mathrm{s}$, then for all $\rho_{\text {emin }} \leq \xi_{1} \leq \rho_{2}$, impacts will occur at what is considered a safe velocity to prevent a bounce in the control. 


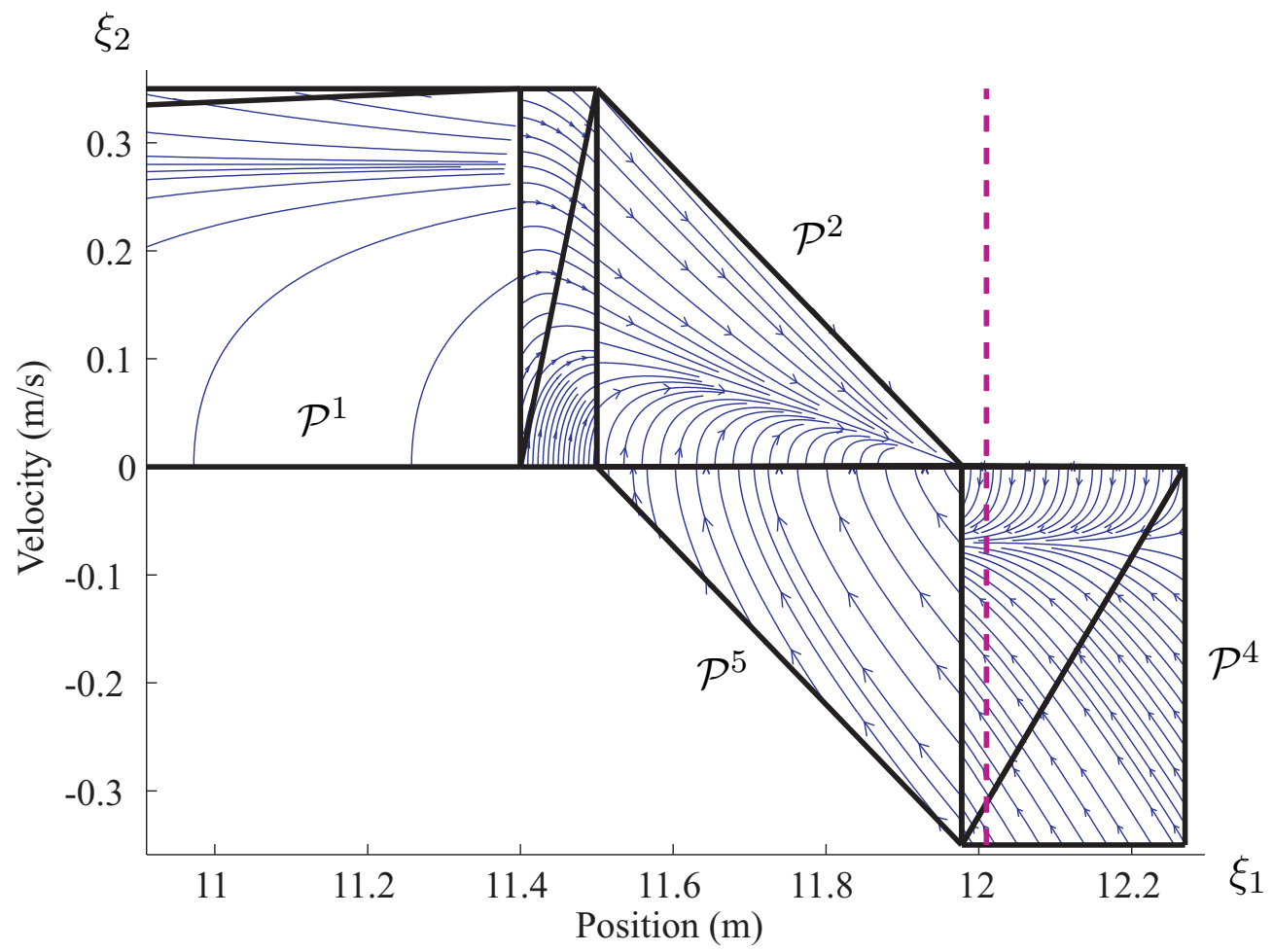

Figure 6.3: Closed-loop vector field of $\mathcal{P}$ under $u_{r c p}$.

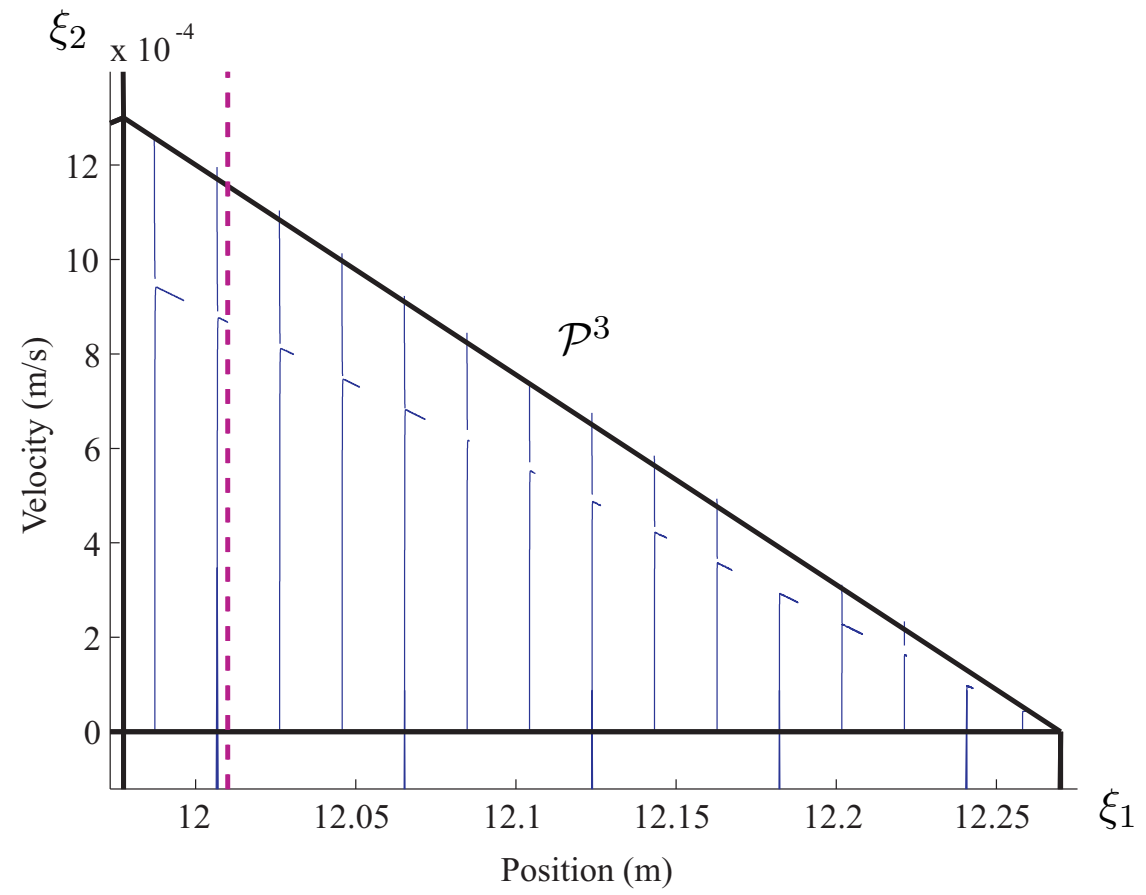

Figure 6.4: Closed-loop vector field of $\mathcal{P}^{3}$ under $u_{r c p}^{10}$. 


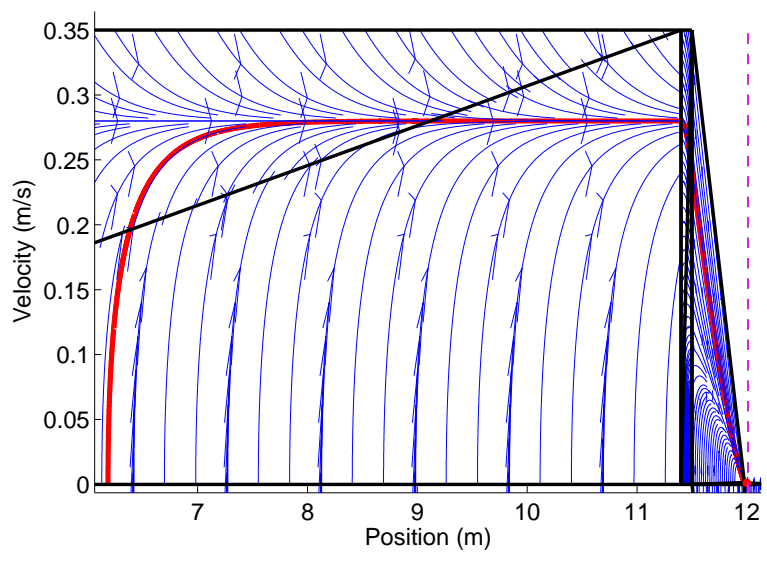

(a)

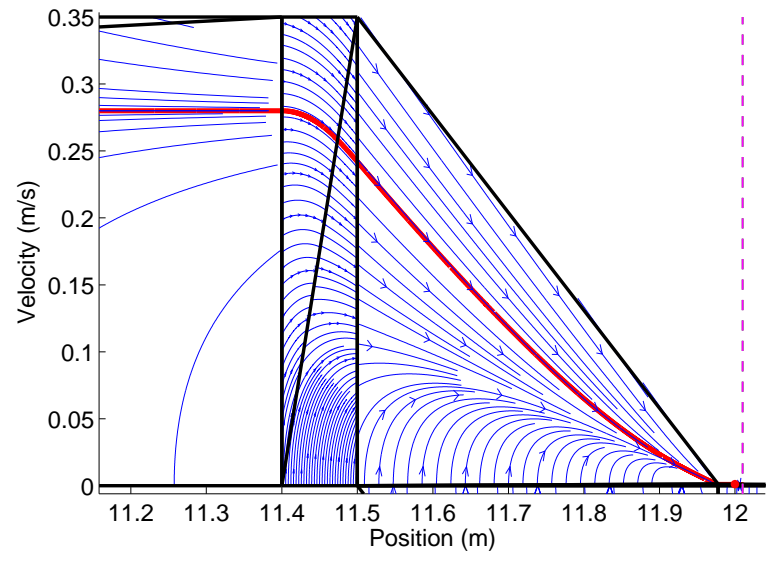

(b)

Figure 6.5: (a) Trajectory of $\xi$ of the first mass in $\mathcal{P}^{1}$. (b) Trajectory of $\xi$ in $\mathcal{P}^{1}$ and $\mathcal{P}^{2}$.

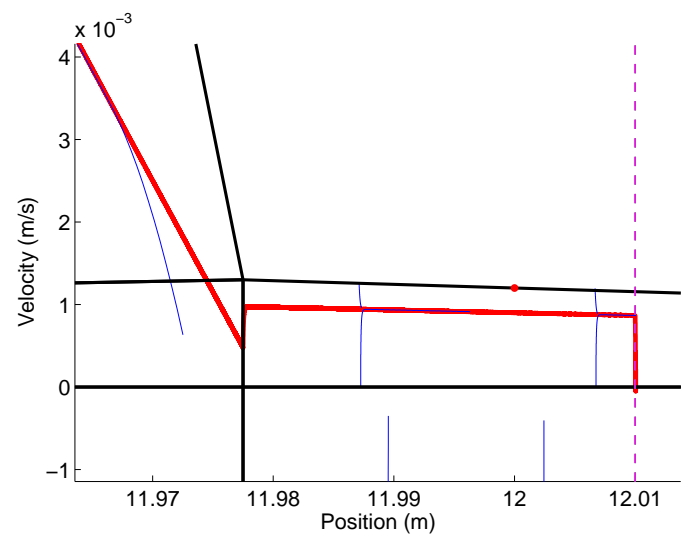

Figure 6.6: Trajectory of $\xi$ of the first mass in $\mathcal{P}^{3}$.

\subsection{Simulations}

In this section we use the hybrid control (6.1) to simulate the full manipulator system and evaluate the proposed design. First, a set of base tests will be performed to demonstrate the desired behaviour under nominal conditions. Then, subsets of the system parameters will be varied beyond their nominal values to investigate the robustness of the design to parameter variation. 


\section{Base Tests}

Using the parameters and controllers generated by the design a set of base tests are conducted for the given problem. First, we set the environment position to $\hat{\rho}_{e}=12.015 \mathrm{~m}$, and since the environment stiffness may fall within a range of values, we simulate three cases: $k_{e}=$ $10^{6}, 10^{7}, 5 \cdot 10^{7} \mathrm{~N} / \mathrm{m}$. For all three tests the manipulator was initialized at rest with joint angles $\left(110^{\circ},-130^{\circ},-10^{\circ}\right)$, which correspond to initial conditions $\left(x_{1}, y_{1}\right)=(6.184,2.086) \mathrm{m}$, $\left(x_{2}, y_{2}\right)=(6.188,2.084) \mathrm{m}$, and $\phi(0)=-30^{\circ}$. Figures 6.5-6.6 depict the reach control phase of the maneuver, where the trajectory of $\xi$ through $\mathcal{P}$ is given by the red curve (we need only show one of the cases here since $k_{e}$ does not affect the motion through $\mathcal{P}$ ). Clearly the reach controllers induce the expected behaviour, ensuring that the safety and liveness constraints of $\mathcal{P}$ are met. In plot (a) the end effector accelerates to a steady-state velocity in $\mathcal{P}^{1}$, while in plot (b) the deceleration process begins as $\xi$ travels through $\mathcal{P}^{2}$. In Figure $6.6, \xi$ transitions from $\mathcal{P}^{2}$ to $\mathcal{P}^{3}$, which is marked by a distinct "jump". This behaviour is a consequence of the discontinuous control design of the reach controllers in this section. This behaviour can be avoided by defining more simplices in the area, and designing the corresponding reach controllers to realise continuous transitions amongst themselves (as is done for the reach controllers of $\mathcal{P}^{1}$ and $\left.\mathcal{P}^{2}\right)$. With $\xi$ in $\mathcal{P}^{3}$, the reach controller $u_{r c p}^{10}$ slowly guides the end effector of the manipulator towards the environment with decreasing velocity until impact is made with velocity $0.9 \mathrm{~mm} / \mathrm{s}$. This is well below the maximum possible impact velocity of $\mu_{s w}=1.2 \mathrm{~mm} / \mathrm{s}$ at $\rho_{\text {emin }}=12 \mathrm{~m}$. Similar behaviour for the second mass states, $\left(x_{2}, \dot{x}_{2}\right)$, is observed in Figure 6.7. The behaviour is more oscillatory, but ultimately the second mass is driven to the environment where an initial impact occurs also at approximately $0.9 \mathrm{~mm} / \mathrm{s}$.

What follows in Figures 6.8-6.10 are the transition and constrained motion phases of the maneuver for $\xi,\left(x_{2}, \dot{x}_{2}\right)$, and sensed force $f_{s}$ for the three tests (ordered from highest to lowest values of $k_{e}$ ). In regards to annotations, the graphs on the left depict in blue the phase lines of the closed-loop force dynamics (4.43e), while on the right the blue waveform represents the sensed force $f_{s}$, the red represents the environment force $f_{e}$, and the black dashed lines represent the switching threshold $f_{s w}=-30 \mathrm{~N}$ and desired force $f_{s}^{d}=-300 \mathrm{~N}$. Referring first to the graphs on the left, plots (a), the common trend among the three simulations is that 


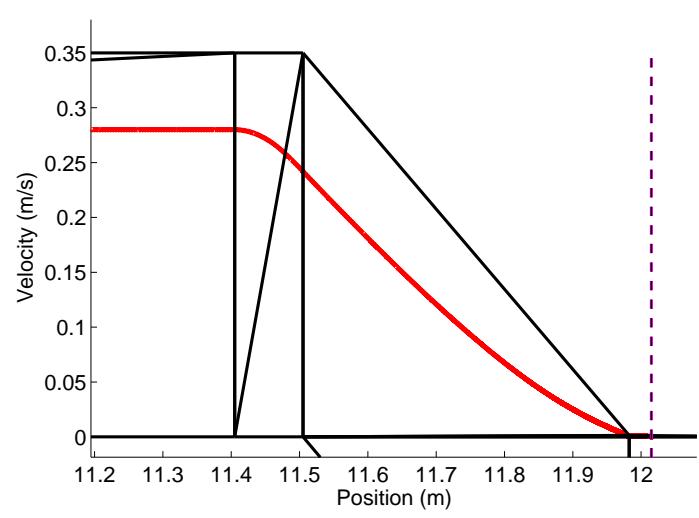

(a)

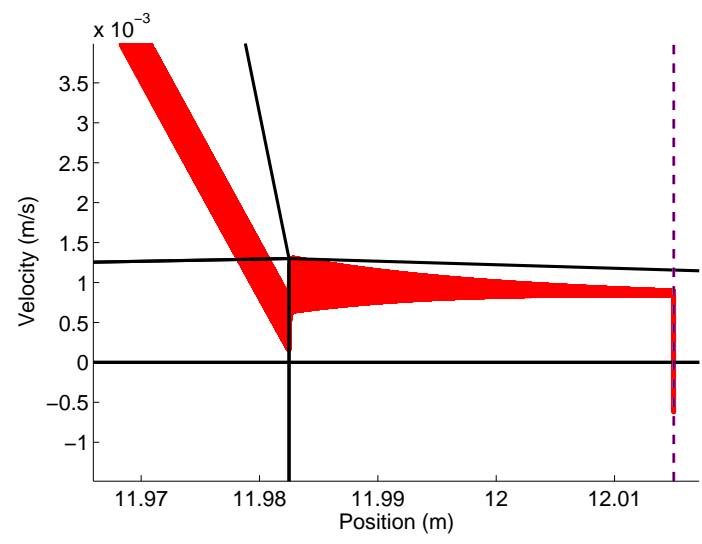

(b)

Figure 6.7: (a) Trajectory of $\left(x_{2}, \dot{x}_{2}\right)$ of the second mass in $\mathcal{P}^{1}$ and $\mathcal{P}^{2}$. (b) Trajectory of $\left(x_{2}, \dot{x}_{2}\right)$ of the second mass in $\mathcal{P}^{3}$.

$u_{r c p}^{10}$ guides $\xi$ to the switching boundary, approximated by a green vertical line, such that $f_{s}$ exceeds the threshold $f_{s w}$. A switch to force control then occurs, and both $\xi$ and the states of the second mass converge to positions associated with $f_{s}^{d}$; moreover the evolution of $\xi$ closely matches the behaviour of the overlaid simplified dynamics. Similarly, in the graphs on the right, plots (c), the sensed force is regulated to $f_{s}^{d}$ without crossing back over $f_{s w}$. In other words, a bumpless transfer occurs. Therefore, the proposed reach controllers, switching law, and constrained controller have collectively achieved the temporal sequence, and obeyed the remaining logic control specifications of Problem 5.1. Other salient features of the graphs are of note. First, the force response is critically damped when $k_{e}=5 \cdot 10^{7} \mathrm{~N} / \mathrm{m}$, as intended, and is more oscillatory for lower values of $k_{e}$; the overshoot is a result of non-zero initial conditions. Second, for high $k_{e}$ the environment force $f_{e}$ in Figure 6.8 (c) is more oscillatory, but is still effectively approximated by $f_{s}$ such that regulation to $f_{s}^{d}$ occurs. As a result of the oscillations the second mass is seen to bounce off of the surface only once in Figure 6.8 (b) but does not disrupt the eventual transition to force control. Also in Figure 6.8(b), we see that the assumption in Chapter 4 that $\ddot{x}_{2} \approx 0$ during contact reasonably holds, as the second mass is held against the environment and the first mass progresses forward.

Supplementary results are also provided for the case where $k_{e}=5 \cdot 10^{7} \mathrm{~N} / \mathrm{m}$, which will only be presented here once since similar results were observed for all tests. In particular, Figure 


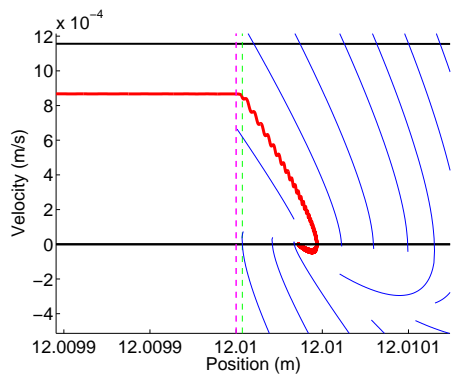

(a)

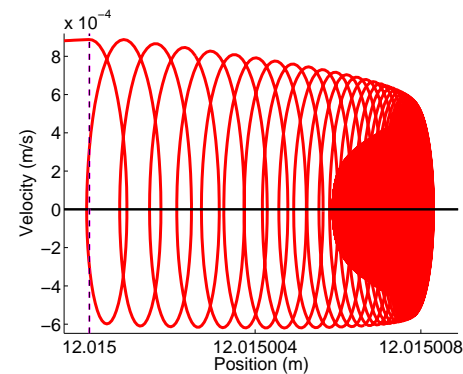

(b)

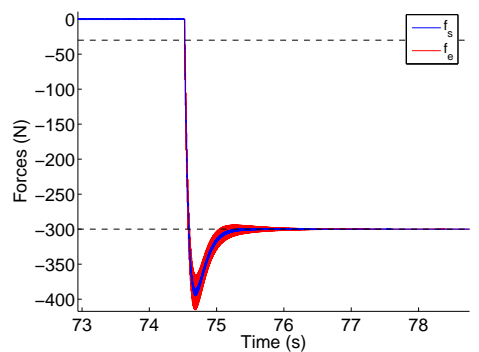

(c)

Figure 6.8: Highest environment stiffness $k_{e}=5 \cdot 10^{7} \mathrm{~N} / \mathrm{m}$ : (a) Constrained trajectory of $\xi$. (b) Constrained trajectory of $\left(x_{2}, \dot{x}_{2}\right)$. (c) Sensed force $f_{s}$ and environmental force $f_{e}$.

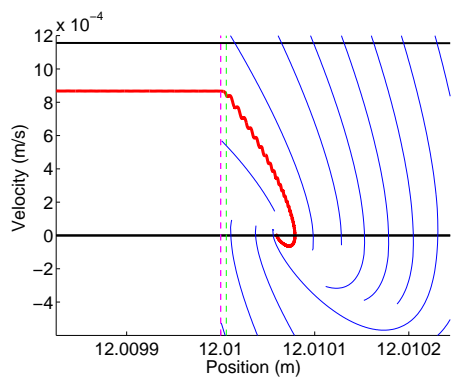

(a)

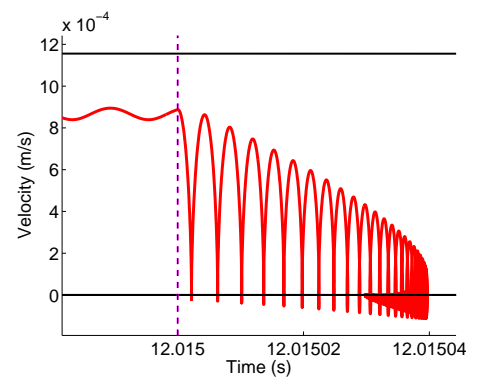

(b)

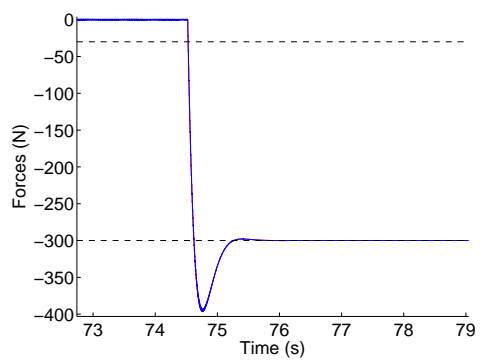

(c)

Figure 6.9: Mid-range environment stiffness $k_{e}=10^{7} \mathrm{~N} / \mathrm{m}$ : (a) Constrained trajectory of $\xi$. (b) Constrained trajectory of $\left(x_{2}, \dot{x}_{2}\right)$. (c) System forces $f_{s}$ and $f_{e}$.

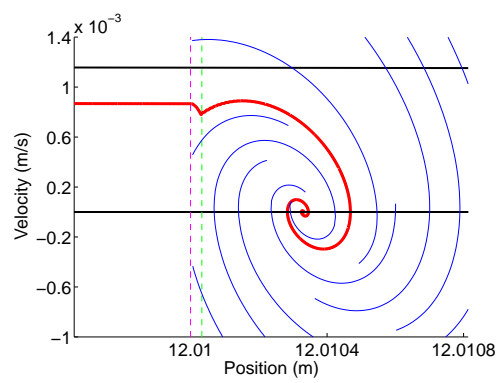

(a)

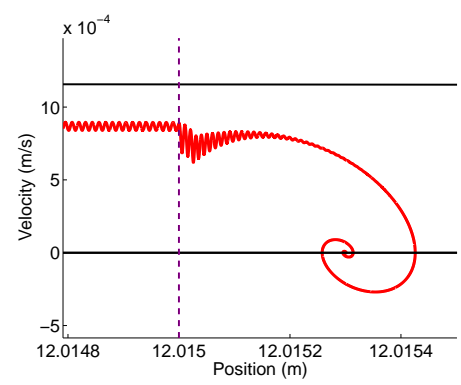

(b)

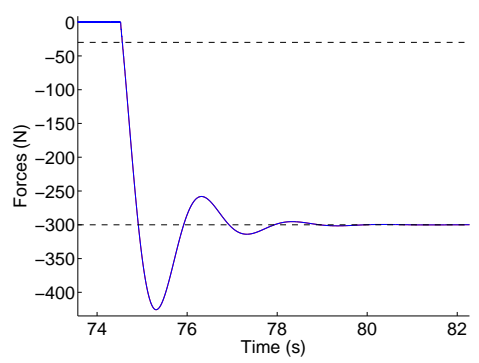

(c)

Figure 6.10: Lowest environment stiffness $k_{e}=10^{6} \mathrm{~N} / \mathrm{m}$ : (a) Constrained trajectory of $\xi$. (b) Constrained trajectory of $\left(x_{2}, \dot{x}_{2}\right)$. (c) System forces $f_{s}$ and $f_{e}$.

6.11 depicts which sub-controller of $u\left(\xi, f_{s}\right)$ is active as a function of time, and it depicts the magnitudes of the joint torques $\tau_{1}, \tau_{2}, \tau_{3}$. In the graph on the left, the control law index is enumerated according to the active reach controller $u_{r c p}^{i}, i \in\{1, \ldots, 10\}$, or the number 11 
when $u_{f}$ is active. Clearly, the particular reach controller of $u\left(\xi, f_{s}\right)$ that is active at any specific time coincides with the behaviour in Figures 6.5-6.6. The inset depicts the spurious switching between $u_{r c p}^{5}$ and $u_{r c p}^{6}$ as alluded to in the previous section. Since the controllers are identical there is no adverse impact (one could collapse the two controller states into one state to eliminate switches in the supervisory control). Lastly, we observe the timing of the maneuver and see that a switch to $u_{f}$ occurs at approximately $74.5 \mathrm{~s}$, where the majority of time was spent in the low-velocity region $\mathcal{P}^{3}$.

Moving on, in the right hand graph are the joint torques used in the feedback linearization $\tau=M(\theta) U_{\theta}-J(\theta)^{T} F_{s}$. Note that an initial spike for $\tau_{2}$ of $-12000 \mathrm{Nm}$ is not displayed for visual purposes. The torque waveforms have a number of features of interest. In particular, the initial spikes lasting from about zero to six seconds are explained by the fact that the manipulator starts in an arbitrary configuration, and so the PD controllers must regulate the task space variables $y_{1}$, and $\phi$ to their reference values, while the reach controllers accelerate the system forward in the $x$-direction. Then, the spike at around 20 s corresponds to the system entering $\mathcal{P}^{2}$ and quickly decelerating the system according to the diagonal safety constraint of Figure 5.2. This behaviour highlights an important trade-off in the design of the region $\mathcal{P}^{2}$, in particular the selection of the position parameter $\rho_{3}$. Referring to Figure 5.2, notice that if $\rho_{3}$ is shifted to the left, then the size of $\mathcal{P}^{2}$ increases, and the slope of the upper diagonal edge becomes less steep. The opposite results occur when $\rho_{3}$ is shifted to the right. This demonstrates a trade-off between liveness (when $\mathcal{P}^{2}$ is small), and safety (when $\mathcal{P}^{2}$ is large). That is, when $\mathcal{P}^{2}$ is large, then the motors need not work as hard to decelerate the system because the reduction in manipulator speed from a maximum of $\mu_{1}$ in $\mathcal{P}^{1}$ to $\mu_{2}$ in $\mathcal{P}^{3}$ can occur over a greater distance. This means that the spike in applied torque as seen in Figure 6.11 will not be as severe. Therefore, $\rho_{3}$ should be chosen to size $\mathcal{P}^{2}$ according to the capabilities of the motors in a real implementation of this design. Finally, the last feature of the torque waveforms is the spike at around $74.5 \mathrm{~s}$, which is when the force controller is activated.

Finally, Figure 6.12 depicts the regulation of the remaining task space variables $\phi$ and $y_{1}$. On the left is the evolution of the joint variables $\theta_{i}$, along with the central magenta line representing $\phi$. As the maneuver takes some time, we clearly achieve early regulation of $\phi$ to $\phi^{d}=0^{\circ}$ to ensure a purely horizontal approach to the environment. On the right is the time 


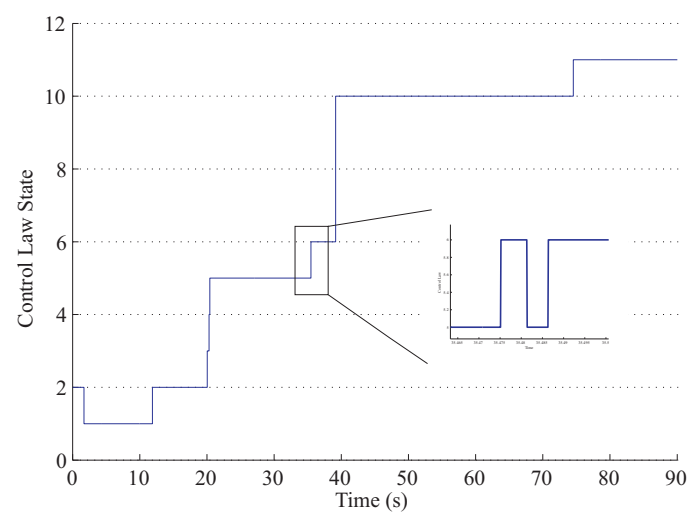

(a)

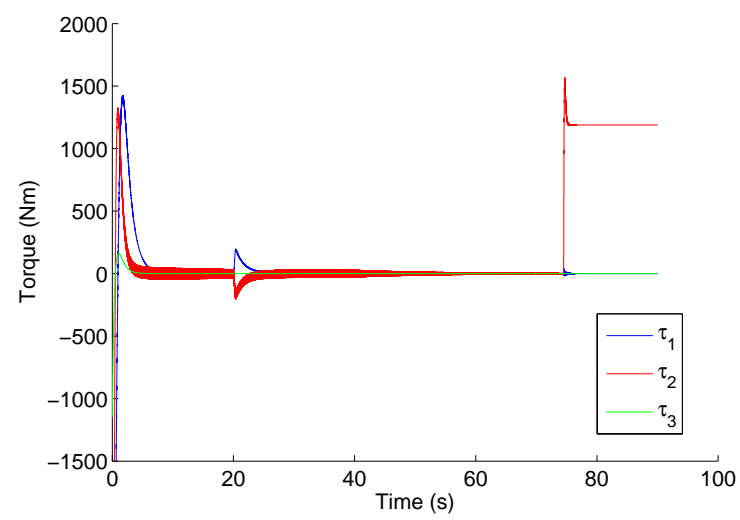

(b)

Figure 6.11: (a) Index of active sub-controller of $u\left(\xi, f_{s}\right)$. (b) Manipulator joint torques $\tau$.

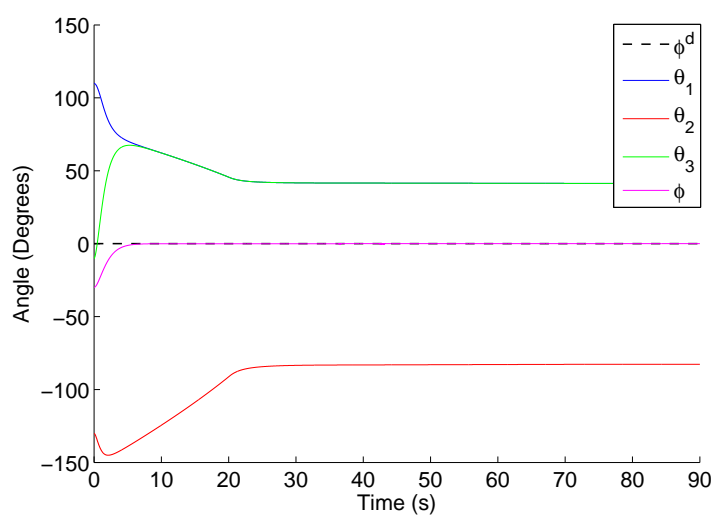

(a)

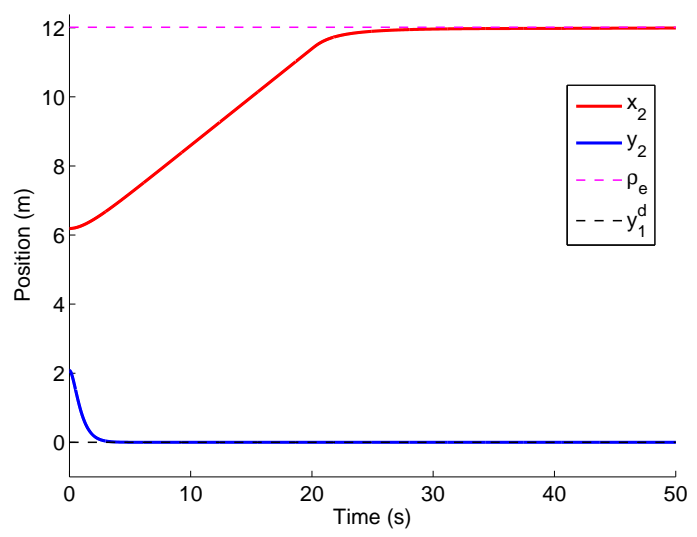

(b)

Figure 6.12: (a) Manipulator joint angles $\theta$ and orientation angle $\phi$. (b) Cartesian coordinates of the second mass $x_{2}$ and $y_{2}$.

evolution of $x_{2}$ and $y_{2}$ of the second mass. Here too we observe quick regulation of $y_{2}$ to the reference $y_{1}^{d}$. We remark that in actual fact $y_{1}$ is regulated to $y_{1}^{d}=0 \mathrm{~m}$, but in conjunction with the regulation of $\phi$ to zero this physically achieves $y_{2} \rightarrow y_{1}^{d}$.

Overall, this set of experiments is considered successful and indicative of the desired behaviour that solves Problem 5.1. In the following, we adjust system parameters to investigate the robustness of the proposed design. The adjustments are categorized into those concerning the proposed control, the environment parameters, and manipulator stiffness. Note that the 


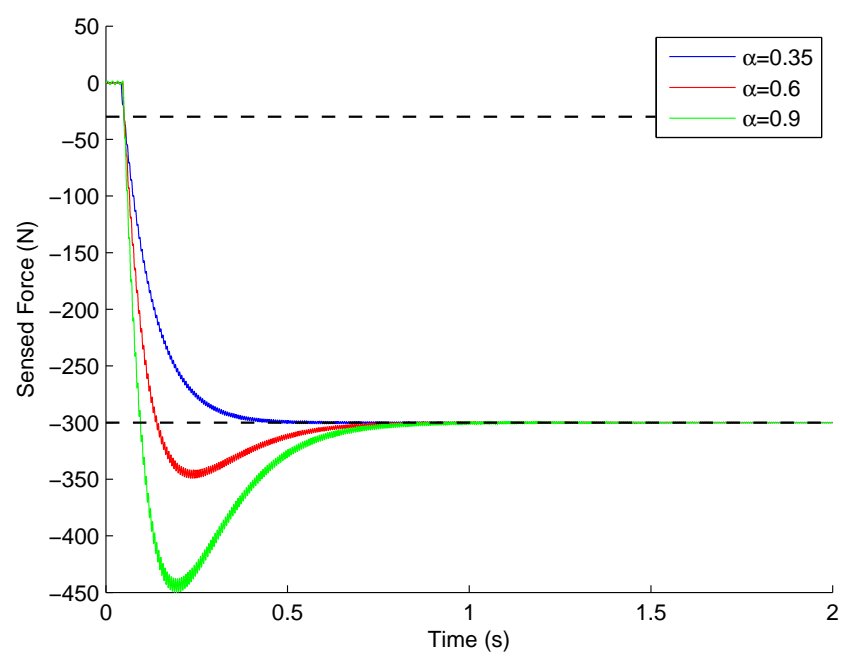

Figure 6.13: Sensed force $f_{s}$ for varying reach control design parameter $\alpha$.

environment stiffness $k_{e}=5 \cdot 10^{7}$ will be used for all subsequent tests unless otherwise stated.

\section{Controller Modifications}

We first investigate how a redesign of reach controller $u_{r c p}^{10}$ and the mass ratio $m_{1} / m_{2}$ of the feedback linearisation affects the nominal system behaviour. Reach controller $u_{r c p}^{10}$ is of particular interest because contact is first made under this controller. Recall that the design of $u_{r c p}^{10}$ requires the selection of vertex controls $u_{6}^{3}, u_{7}^{3}$, and $u_{8}^{3}$. Our design requires $u_{8}^{3}=0$ to place an equilibrium at $v_{8}$, while $u_{7}^{3}>0$ must satisfy (5.6) of Lemma 5.1. This leaves $u_{6}^{3}<0$ free to select. Let $\alpha \in \mathbb{R}$ be a ratio defined as $\alpha:=\frac{u_{7}^{3}}{u_{7}^{3}-u_{6}^{3}}$. Therefore, assuming $u_{7}^{3}$ is fixed, by increasing the magnitude of $u_{6}^{3}$, i.e. pushing down harder from $v_{6}$, one decreases $\alpha$. Recall that in $\mathcal{P}^{3}$ the velocity $\xi_{2}$ generally decreases linearly with position $\xi_{1}$. It can be shown that the slope of this decrease is related to $\alpha$. The result is that for smaller $\alpha$ trajectories in $\mathcal{P}^{3}$ travel slower, and the impact velocity is reduced. Figure 6.13 depicts the response $f_{s}$ for $\alpha=0.35,0.6,0.9$ (which have been aligned), which correspond respectively to reach controllers

$u_{r c p}^{10}=\left[\begin{array}{ll}-240 & -154305\end{array}\right] \xi+2945, u_{r c p}^{10}=\left[\begin{array}{ll}-240 & -90011\end{array}\right] \xi+2945, u_{r c p}^{10}=\left[\begin{array}{lll}-240 & -60007\end{array}\right] \xi+2945$.

From smallest to largest the impact velocities for these tests were: $0.405 \mathrm{~mm} / \mathrm{s}, 0.7 \mathrm{~mm} / \mathrm{s}$, and $1.06 \mathrm{~mm} / \mathrm{s}$. All three responses are critically damped, but due to differing initial conditions, 


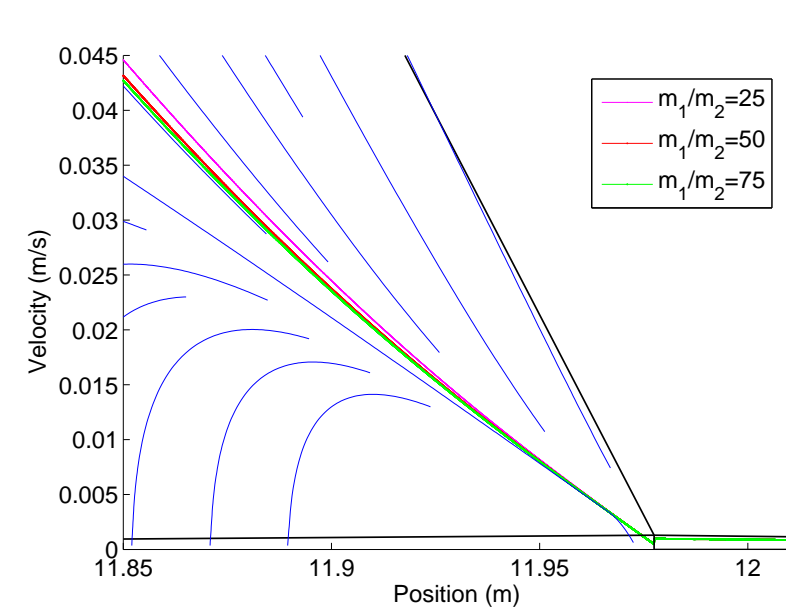

(a)

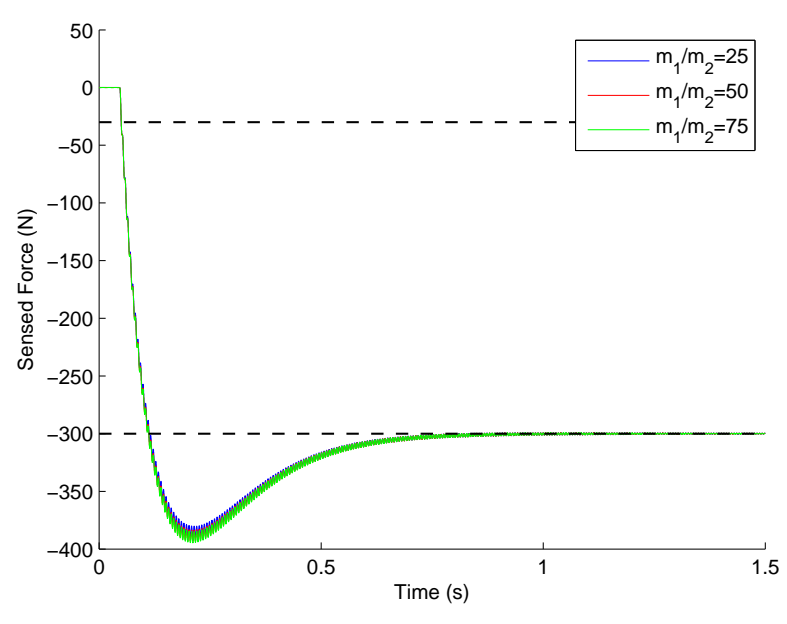

(b)

Figure 6.14: (a) Trajectories $\xi$ of the first mass in $\mathcal{P}^{2}$ and $\mathcal{P}^{3}$ for different mass ratios. (b) The sensed force $f_{s}$ for different mass ratios.

overshoots are clearly visible for larger values of $\alpha$ (note, in the base test we have $\alpha=0.75$ which yields a peak force of $\left.f_{s}=-390 \mathrm{~N}\right)$. We conclude that, in addition to achieving bumpless transfer from reach to force control, adequate design of $u_{r c p}^{10}$ can also limit the peak contact force. The caveat is that a reduced $\alpha$ leads to longer maneuver times.

Next we vary the mass ratio $m_{1} / m_{2}$. In particular, $m_{1}$ was selected to satisfy $m_{1} / m_{2}=$ $75,50,25$, and then the reach controllers and force controller were redesigned. The resulting reach and force controllers saw their parameters scaled by $75 \%, 50 \%$, and $25 \%$ respectively from the nominal controllers. The exception is reach controller $u_{r c p}^{10}$ of $\mathcal{P}^{3}$, which remained constant, since it was designed to address equilibria and reaching the switching boundary (see Lemma 5.1 for details). First, we examine the effects of the mass ratio on the reach control phase of the maneuver. Figure 6.14 (a) depicts three overlaid trajectories of $\xi$ for the different mass ratios as they travel through a portion of $\mathcal{P}^{2}$ enroute to $\mathcal{P}^{3}$. At higher velocity we observe a slight difference in the trajectories, but they ultimately converge upon entering $\mathcal{P}^{3}$. In effect we observe that the reach controllers sufficiently guide the end effector of the first mass through $\mathcal{P}$ for mass ratios lower than that of the base test. In regards to constrained motion, Figure 6.14 (b) depicts the sensed force response $f_{s}$ overlaid for all three cases. Again we observe little difference between cases, as a bumpless transfer to force control occurs for all three tests. These 


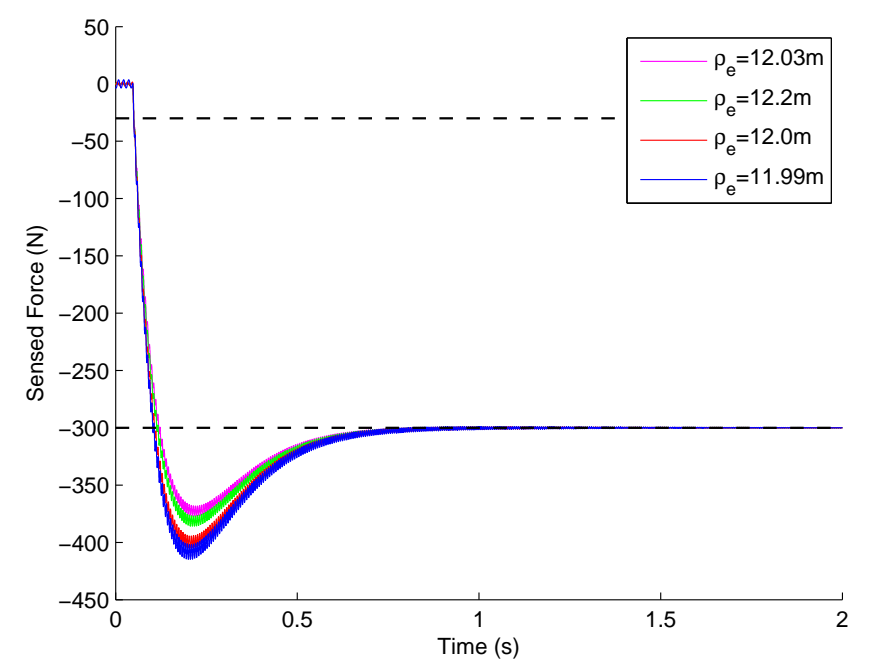

Figure 6.15: Sensed force $f_{s}$ for varying environment position $\rho_{e}$.

tests therefore validate the modelling assumptions of Section 4.2, and the design methodology, whereby reach controllers are designed with respect to the first mass.

\section{Environment Variations}

Now we simulate the manipulator system when the environment location has changed (both within and beyond its expected range), and when the expected environment stiffness is violated. Starting with position variation, the environment was positioned at the extremes of its assumed range, i.e. $\hat{\rho}_{e}=\hat{\rho}_{\text {emax }}=12.025 \mathrm{~m}$, and $\hat{\rho}_{e}=\hat{\rho}_{\text {emin }}=12.005 \mathrm{~m}$, and at positions outside of this range, i.e. $\hat{\rho}_{e}=12.035 \mathrm{~m}$, and $\hat{\rho}_{e}=11.995 \mathrm{~m}$. Figure 6.15 depicts the response of the sensed force $f_{s}$ for all four tests. Since the state space $\mathcal{P}$ and reach control $u_{r c p}$ were designed with knowledge of a nominal variation in $\hat{\rho}_{e}$ it is not surprising that for the cases $\hat{\rho}_{\text {emax }}$ and $\hat{\rho}_{\text {emin }}$ (the two middle waveforms) that the reach controller in $\mathcal{P}^{3}$ successfully guides trajectories to the switching line and bumpless transfer to force control occurs.

Now we examine why the off-nominal simulations were successful. The case where $\hat{\rho}_{e}=$ $12.035 \mathrm{~m}$ works for two reasons: i) the selection of $\rho_{2}$ from (5.5) is sufficiently large to account for variations in the maximum position of the environment, i.e. there exist $\epsilon>0$, such that

$\rho_{2}$ satisfies $\rho_{2}>\rho_{\text {emax }}+\epsilon+\frac{\left|f_{s w}\right|}{\bar{k}_{\text {min }}}>\rho_{\text {emax }}+\frac{\left|f_{s w}\right|}{\bar{k}_{\text {min }}}$; ii) the control $u_{7}^{3}$ is also chosen to yield similar margin with respect to the constraint (5.6). This means the reach controller is strong enough to drive trajectories to the switching boundary in the presence of location variation. 


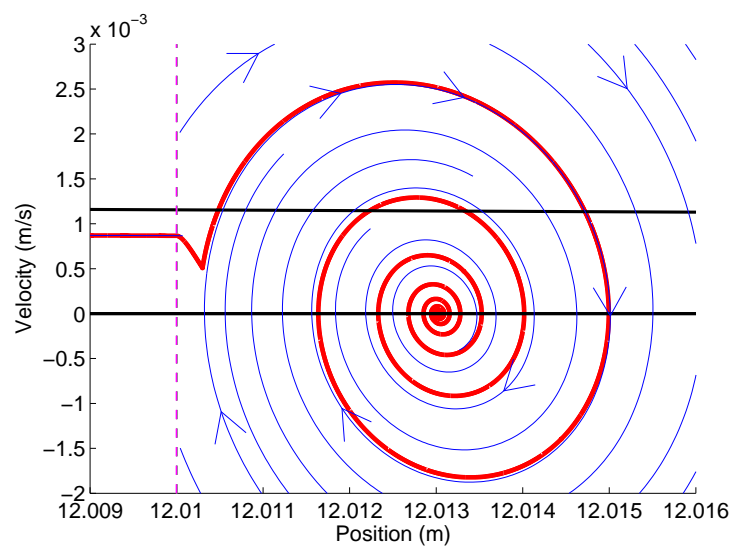

(a)

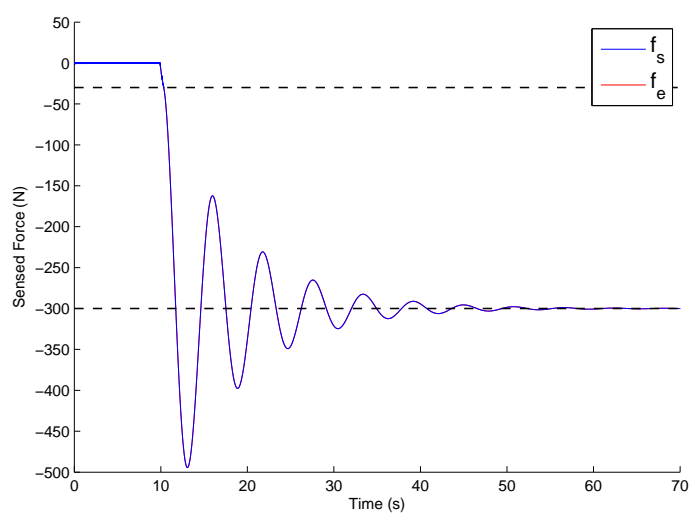

(b)

Figure 6.16: Below nominal environment stiffness $k_{e}=10^{5} \mathrm{~N} / \mathrm{m}$ : (a) Constrained trajectory $\xi$ of the first mass. (b) System forces $f_{s}$ and $f_{e}$.

Conversely when $\hat{\rho}_{e}=12.005 \mathrm{~m}$, reaching the switching boundary is not a concern. Instead, we are interested in the impact velocity. In the simulation the impact velocity is $0.932 \mathrm{~mm} / \mathrm{s}$, which is less than the least upper bound $\bar{\mu}_{s w}=1.3 \mathrm{~mm} / \mathrm{s}$. By Lemma 5.3 this implies that after impact and when a subsequent switch to force control occurs, the states $\xi$ have entered an invariant set with respect to the force dynamics (4.43e); therefore a return to reach control is not possible. Consequently, by designing the region $\mathcal{P}^{3}$ correctly, in this case selecting $\mu_{2}$ close to $\mu_{s w}$ or $\bar{\mu}_{s w}$, impact velocities can be kept within safe levels to obtain the desired switching behaviour even when the environment position is uncertain.

Additionally, if we examine the force responses themselves a clear trend emerges where the peak contact force decreases as $\rho_{e}$ increases. This relationship is simple to grasp: since the velocity $\xi_{2}$ decreases linearly in $\mathcal{P}^{3}$, the resulting impact velocity is larger for closer environments, which leads to a difference in initial conditions once the force controller is activated. From largest to smallest the impact velocities for these tests were: $0.932 \mathrm{~mm} / \mathrm{s}, 0.9 \mathrm{~mm} / \mathrm{s}, 0.834 \mathrm{~mm} / \mathrm{s}$, and $0.8 \mathrm{~mm} / \mathrm{s}$. Therefore, for this design, accurate knowledge of the environment location can be used to place bounds on the peak contact force.

Next, we test our initial design for environment stiffness $k_{e}$ beyond the nominal range, i.e. $k_{e}=10^{5} \mathrm{~N} / \mathrm{m}$, and $k_{e}=10^{8} \mathrm{~N} / \mathrm{m}$. Figure 6.16 depicts the transition and constrained motion phases of $\xi$ and $f_{s}$ when $k_{e}=10^{5} \mathrm{~N} / \mathrm{m}$. Inspite of the lower than expected $k_{e}$ a bumpless transfer 


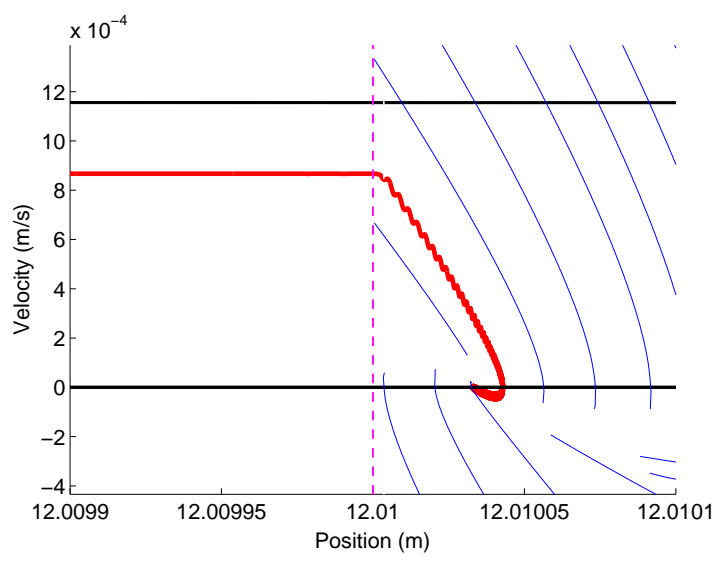

(a)

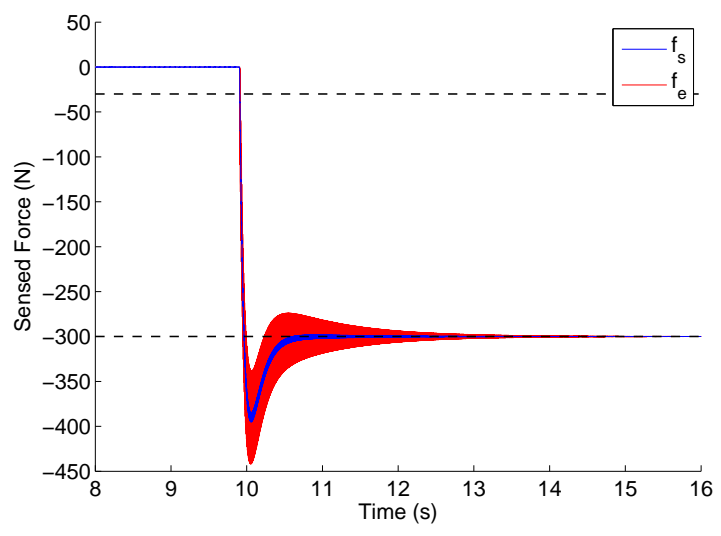

(b)

Figure 6.17: Above nominal environment stiffness $k_{e}=10^{8} \mathrm{~N} / \mathrm{m}$ : (a) Constrained trajectory $\xi$ of the first mass. (b) System forces $f_{s}$ and $f_{e}$.

to force control occurs for reasons analogous to those from the previous tests. Specifically, $\rho_{2}$ and $u_{7}^{3}$ are chosen to allow for variation in the term $\frac{\left|f_{s w}\right|}{\overline{k_{m i n}}}$ that appears in (5.5) and (5.6) respectively. This ensures that trajectories reach the switching boundary. Moreover, the velocity $\xi_{2}$ at this instance is less than $\mu_{s w}$ and $\bar{\mu}_{s w}$. As a result an invariant set, as described in Lemmas 5.2 and 5.3 will have been entered, and the stable force controller regulates $f_{s}$ to $f_{s}^{d}$ (albeit the actual force dynamics are more oscillatory and take longer to converge).

Figures 6.17 and 6.18 depict the case where $k_{e}=10^{8} \mathrm{~N} / \mathrm{m}$. In the former we observe the convergence of $\xi$ to the equilibrium $\xi_{f}^{d}=\left(\rho_{f}^{d}, 0\right)$, and of $f_{s}$ to $f_{s}^{d}=-300 \mathrm{~N}$. The fundamental reason why this case is successful is because the impact velocity is less than $\bar{\mu}_{s w}$. In particular, suppose we consider the actual effective spring constant for this test: $\bar{k}^{\prime}=\frac{k_{s} \cdot 10^{8}}{k_{s}+10^{8}}$. One can replace $\bar{k}_{\text {max }}$ with $\bar{k}^{\prime}$ in Lemma 5.3 to generate an invariant set and confirm the simulated result. However, in Figure 6.18 we view in more detail the consequences of the increased environment stiffness on the second mass. In plot (a) the second mass bounces almost twice before maintaining contact. In plot (b) we explicitly observe the evolution of $f_{s}$ as it crosses the $-30 \mathrm{~N}$ threshold. Clearly the motion of the second mass influences the measurement $f_{s}$, but does not negatively interfere with the switching and regulation process. Therefore, from these tests it is clear that the proposed design solves Problem 5.1 for cases where the environment properties exceed their nominal ranges. 


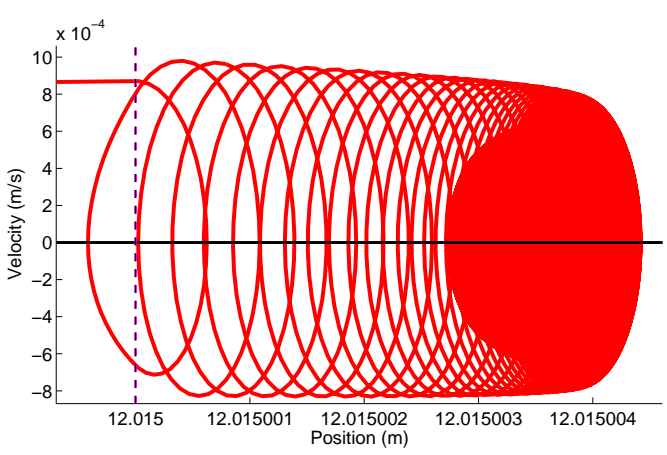

(a)

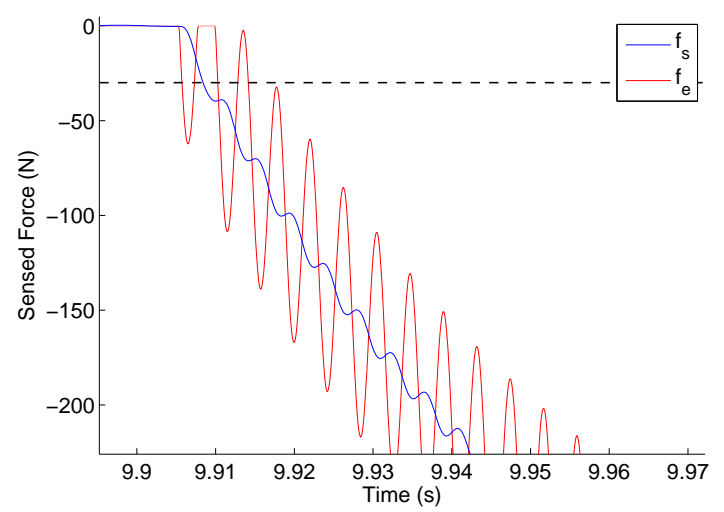

(b)

Figure 6.18: Above nominal environment stiffness $k_{e}=10^{8} \mathrm{~N} / \mathrm{m}$ : (a) Constrained trajectory $\left(x_{2}, \dot{x}_{2}\right)$ of the second mass. (b) System forces $f_{s}$ and $f_{e}$ close to the switching boundary.

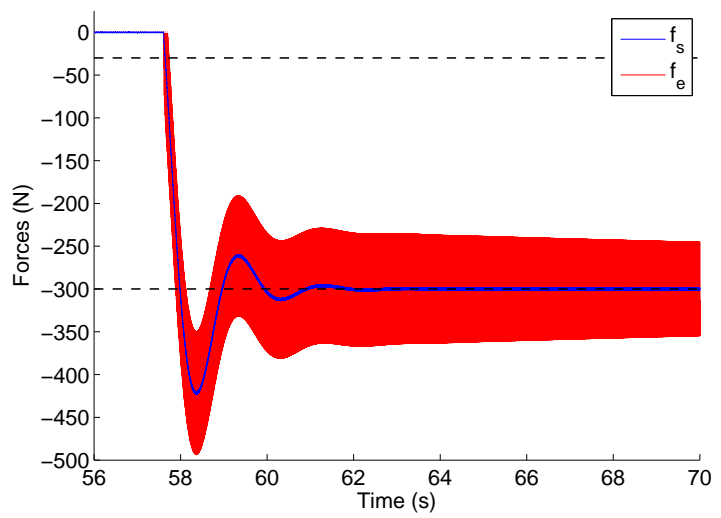

(a)

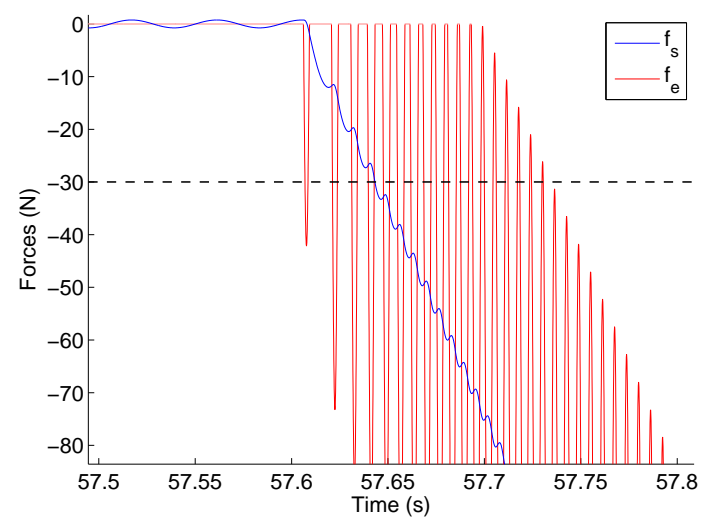

(b)

Figure 6.19: Below nominal sensor stiffness $k_{s}=5 \cdot 10^{5} \mathrm{~N} / \mathrm{m}$ : (a) System forces $f_{s}$ and $f_{e}$. (b) System forces $f_{s}$ and $f_{e}$ close to the switching boundary.

\section{Manipulator Variation}

Assuming again the base parameters, we explore a variation of the manipulator system in the form of a degraded sensor stiffness. Figure 6.19 depicts a scenario where the sensor stiffness has softened to $k_{s}=5 \cdot 10^{5} \mathrm{~N} / \mathrm{m}$ such that the ratio $k_{e} / k_{s}=100$ is quite large. The extreme difference renders the manipulator much more compliant, which leads to extremely oscillatory behaviour in the second mass, and likewise the environmental force depicted in red in plot (a). In plot (b), the oscillations lead to excessive bouncing of the second mass during the first second of impact 
as observed. However, the reach controller in $\mathcal{P}^{3}$ still ensures that the switching boundary is reached, and a bumpless transfer to force control occurs (again the reasoning follows from that of the previous tests). It is noted however that while adequate force regulation of $f_{s}$ occurs, the behaviour of the second mass is far from ideal. This is most likely the consequence of the modelling approach adopted, whereby the second mass is left 'dangling' off of the main manipulator body.

Overall, these simulations demonstrate the effectiveness of the proposed control methodology in solving Problem 5.1 for this simplified system, even in the face of parameter variation. The next step is to adapt the approach to the SPDM. The simulations performed here provide the foundation for those that will be conducted for the SPDM in Chapter 8. 


\section{Chapter 7}

\section{Special Purpose Dextrous Manipulator}

Our focus now turns to the design and simulation of an environment interaction task with a real manipulator system, the Special Purpose Dextrous Manipulator (SPDM). First though, we present in this chapter the dynamical model for the SPDM. The chapter begins with a derivation of a nominal model that is then augmented. Then the SPDM is simplified and adapted to the system formulation given in Chapter 4 for the purpose of control design.

\subsection{SPDM Modelling}

We present here a truncated version of the model derivation from [5], which also deals with the SPDM. Note that the SPDM simulator itself was also a valuable resource for understanding the model and SPDM structure. The SPDM consists of a chain of rigid bodies connected primarily by 1-Degrees of Freedom (DOF) joints. As such, the presentation here first addresses the nature of a single rigid body, and later connects the bodies in a chain to form the manipulator. The SPDM consists of nine bodies $B_{i}, i \in\{0,1, \ldots, 8\}$; the first eight bodies represent the base and seven links connected by seven joints which provide the arm with 7-DOF movement. The last body represents the tip of the manipulator attached through a 6-DOF force/moment sensor (FMS). Let $\tilde{\theta} \in \mathbb{R}^{7}$ be the vector of the seven actuated joints, with elements belonging to $S^{1}$. Moreover, let $\delta \in \mathbb{R}^{6}$ be the vector of FMS joints, where the first three elements belong to $\mathbb{R}$ 


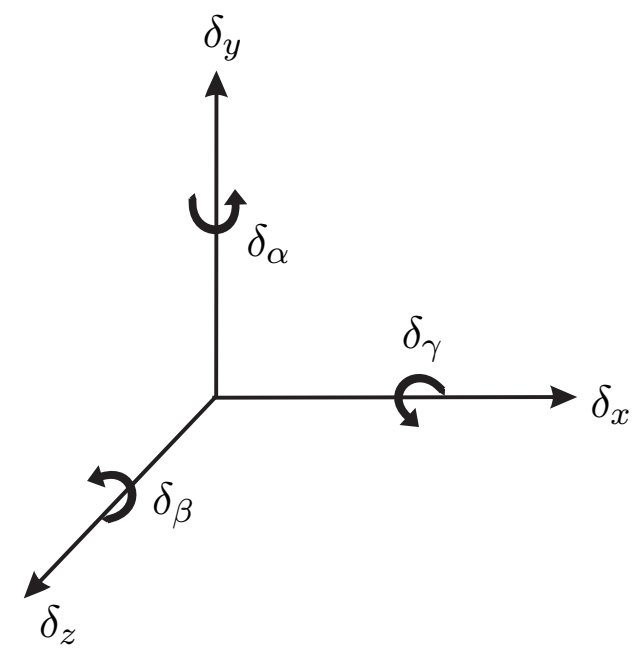

Figure 7.1: Notation and directions for the FMS frame.

(representing displacements along the three Cartesian axes) and the last three elements belong to $S^{1}$ (representing twists about the same axes); Figure 7.1 depicts the FMS variables on a set of axes. Then we define the vector of generalized coordinates $\tilde{q} \in \mathbb{R}^{13}$ as

$$
\tilde{q}:=\left[\begin{array}{ll}
\tilde{\theta} & \delta
\end{array}\right]^{T} \quad \tilde{\theta}:=\left[\begin{array}{llll}
\theta_{1} & \theta_{2} & \ldots & \theta_{7}
\end{array}\right]^{T} \quad \delta:=\left[\begin{array}{llllll}
\delta_{x} & \delta_{y} & \delta_{z} & \delta_{\gamma} & \delta_{\alpha} & \delta_{\beta}
\end{array}\right]^{T}
$$

\subsubsection{Nominal Model}

The first stage of the modelling process aims at generating the simplest dynamical model for the SPDM. Such a system consists of terms representing the acceleration, control input, and external force.

\section{Rigid Body $B_{i}$}

We begin the modelling by describing the essential notation and dynamics associated with a single body $B_{i}$. Figure 7.2 illustrates a general body $B_{i}$ as well as the associated coordinate frames and vectors used in this development. Away from any body we assume a non-accelerating coordinate frame, called the inertial frame $F_{I}$, to which absolute motions are referenced. On each body define the local frame $F_{i}$ with origin $O_{i}$, the location of the joint $i$ that connects $B_{i}$ and $B_{i-1}$. Similarly, define the fixed frame $F_{i \mp 1}$ with origin $O_{i \mp 1}$ to which $B_{i+1}$ is attached at joint $i+1$. Lastly, the frame $F_{i+1}$ which is the local frame of body $B_{i+1}$, will also attach at joint 


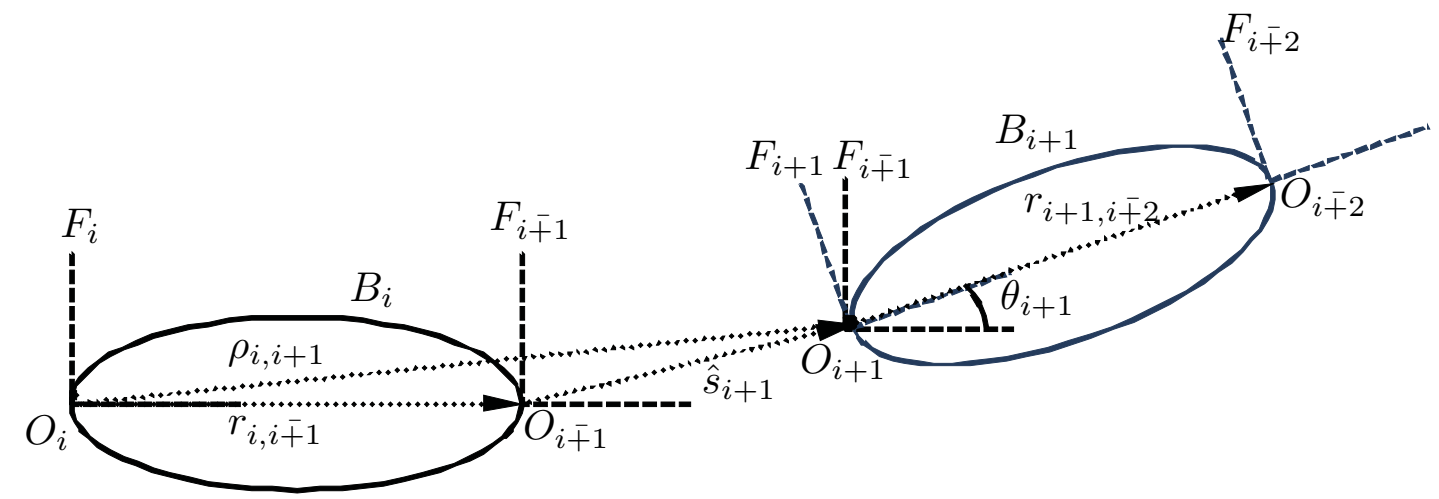

Figure 7.2: Bodies $B_{i}$ and $B_{i+1}$, and associated notation [5].

$i+1$. We mention here that a rotation matrix $C_{i+1}^{i}, i \in\{0, \ldots, 7\}$ is used to transform coordinates from frame $F_{i+1}$ to $F_{i}$. For $i \in\{0, \ldots, 6\}$ the rotation has 1-DOF about some Cartesian axis defined by an angle $\theta_{i+1}$. For $i=7$, the FMS body, the rotation has 3-DOF represented by a sequential set of rotations defined by the angles $\delta_{\beta}, \delta_{\alpha}, \delta_{\gamma}$, such that $C_{8}^{7}=C_{\delta_{\gamma}} C_{\delta_{\alpha}} C_{\delta_{\beta}}$ [38]. Connecting the origin $O_{i}$ to $O_{i \overline{+} 1}$ is the vector $r_{i, i \mp 1} \in \mathbb{R}^{3}$, defined in $F_{i}$. Additionally, the vector $\hat{s}_{i+1} \in \mathbb{R}^{3}$, defined in $F_{i+1}$, is an interbody translation connecting $O_{i \overline{+} 1}$ to $O_{i+1}$. Therefore, let $\rho_{i, i+1} \in \mathbb{R}^{3}$ be the vector from $O_{i}$ to $O_{i+1}$, defined in $F_{i}$, given by

$$
\rho_{i, i+1}=r_{i, i \overline{+} 1}+C_{i+1}^{i} \hat{s}_{i+1}
$$

Each body is characterized by ten inertia parameters [5]: $m_{i}$ is the mass of the body, $c_{i}=\left[\begin{array}{lll}c_{i, x} & c_{i, y} & c_{i, z}\end{array}\right]^{T}$ is the first moment of mass relative to $O_{i}$, and $\bar{J}_{i} \in \mathbb{R}^{3 \times 3}$ is the second moment of mass about $O_{i}$. These parameters are collected into a matrix for each body

$$
M_{i}:=\left[\begin{array}{cc}
m_{i} I_{3} & -c_{i}^{\times} \\
-c_{i}^{\times} & \bar{J}_{i}
\end{array}\right], M_{i} \in \mathbb{R}^{6 \times 6},
$$

where $I_{3}$ is the identity matrix with dimension 3 , and $(\cdot)^{\times}$is the skew-symmetric operator defined in (4.2).

Let $\overrightarrow{v_{i}}$ represent the absolute linear velocity of $O_{i}$, and $\vec{\omega}_{i}$ the absolute angular velocity of $F_{i}$, where the term absolute will reference quantities to the inertial frame $F_{I}$. Then let $v_{i}$ and $\omega_{i}$ be their associated $3 x 1$ coordinates for $B_{i}$ expressed in frame $F_{i}$. Finally, let $\mathcal{V}_{i}$ be the generalized 
velocity of $B_{i}$, which is defined from the absolute quantities as

$$
\mathcal{V}_{i}:=\left[\begin{array}{l}
v_{i} \\
\omega_{i}
\end{array}\right]
$$

Two types of forces are said to act on a body $B_{i}[5]$ : i) a generalized total external and internal force $f_{i T}$, and ii) a generalized nonlinear inertial force $f_{i I}$. Each quantity is represented by a $6 \times 1$ column matrix consisting of three force components applied at $O_{i}$ and three torque components applied about $O_{i}$. Using the various properties and quantities just described the dynamical expression for the generalized motion of a body $B_{i}$ is

$$
M_{i} \dot{\mathcal{V}}_{i}=f_{i T}+f_{i I}, \quad i \in\{0,1, \ldots, 8\}
$$

We now define interbody properties which will commonly be labelled with the accent ${ }^{\wedge}$. Let the relative generalized velocity of $B_{i+1}$ relative to $B_{i}$ be denoted

$$
\hat{\mathcal{V}}_{i+1}:=\left[\begin{array}{c}
\hat{v}_{i+1} \\
\hat{\omega}_{i+1}
\end{array}\right]
$$

which consists of the translational component $\hat{v}_{i+1} \in \mathbb{R}^{3}$, and angular component $\hat{\omega}_{i+1} \in \mathbb{R}^{3}$. In [5] it is shown that the relationship between generalized velocities $\mathcal{V}_{i}$ and $\mathcal{V}_{i+1}$, expressed in frame $F_{i+1}$, is given by

$$
\mathcal{V}_{i+1}:=T_{i}^{i+1} \mathcal{V}_{i}+G_{i+1, i+1} \hat{\mathcal{V}}_{i+1}, \quad i \in\{0,1, \ldots, 7\}
$$

where $T_{i}^{i+1} \in \mathbb{R}^{6 \times 6}$ and $G_{i+1, i+1} \in \mathbb{R}^{6 \times 6}$ are defined as

$$
T_{i}^{i+1}:=\left[\begin{array}{cc}
C_{i}^{i+1} & -C_{i}^{i+1}\left(\rho_{i, i+1}\right)^{\times} \\
0 & C_{i}^{i+1}
\end{array}\right], \quad G_{i+1, i+1}:=\left[\begin{array}{cc}
I_{3} & -\left(\hat{s}_{i+1}\right)^{\times} \\
0 & I_{3}
\end{array}\right]
$$

The matrix $T_{i}^{i+1}$ represents the interbody transformation matrix from $B_{i}$ to $B_{i+1}$, while the matrix $G_{i+1, i+1}$ transforms $\hat{\mathcal{V}}_{i+1}$ to the inertial frame (but the product is still expressed in 
$\left.F_{i+1}\right)$. The actuated joints $\tilde{\theta}$ connect neighbouring bodies directly so no translational motion between them exists. Conversely, for the FMS joint, we will define a non-zero displacement in the $x$-direction, associated with the compression variable $\delta_{x}$. As a result, a non-zero force detected in the $x$-direction by the FMS translates to a non-zero $\delta_{x}$ which then corresponds to a displacement between $B_{7}$ and $B_{8}$ (this approach diverges from the standard SPDM simulator, which does not incorporate this type of behaviour [38]). This desired behaviour is manifested in the system model as follows:

$$
\begin{aligned}
i \in\{0, \ldots, 6\}: & \hat{s}_{i+1}=(0,0,0) \Longrightarrow G_{i+1, i+1}:=I_{6} \\
i=7: & \hat{s}_{i+1}=\left(\delta_{x}, 0,0\right) \Longrightarrow G_{8,8}:=\left[\begin{array}{cc}
I_{3} & -\left(\hat{s}_{i+1}\right)^{\times} \\
0 & I_{3}
\end{array}\right] .
\end{aligned}
$$

Next the number and direction(s) of the DOF to be applied at each joint of the manipulator is specified using a projection matrix $P_{i}$ and its complement $Q_{i}$ [39]. In particular, for a single joint $i$ one may define a generalized interbody force between bodies $B_{i-1}$ and $B_{i}$, denoted $\hat{f}_{i} \in \mathbb{R}^{6}$, which is decomposed into control force $f_{i c}$ and constraint force $f_{i o}$ using $P_{i}$ and $Q_{i}$ :

$$
\hat{f}_{i}:=-P_{i} f_{i c}-Q_{i} f_{i o}
$$

The $n \times m$ matrix $P_{i}$ is defined for each joint where $n$ is the total number of DOF available (typically six), while $m$ is equal to the actual DOF attributed to the joint. As mentioned the SPDM consists of nine bodies, which are connected by eight joints. The seven actuated joints grant full access to any physical configuration with respect to displacements along and orientations about the three cartesian axes. The characteristics of the joints are compiled in Table 7.1. In order, the joint acronyms correspond to shoulder roll, shoulder yaw, shoulder pitch, elbow pitch, wrist pitch, wrist yaw, and wrist roll. The definitions of the joints in the table yield the following projection matrices [38]:

Table 7.1: Manipulator Joints

\begin{tabular}{|c|c|c|c|c|c|c|c|c|c|}
\hline Joint Number & 0 & 1 & 2 & 3 & 4 & 5 & 6 & 7 & FMS \\
\hline Joint Name & 0 & SR & SY & SP & EP & WP & WY & WR & FMS \\
Rotation Axis & NA & $\mathrm{x}$ & $\mathrm{y}$ & $\mathrm{z}$ & $\mathrm{z}$ & $\mathrm{z}$ & $\mathrm{y}$ & $\mathrm{x}$ & 6 -DOF \\
\hline
\end{tabular}




$$
\begin{gathered}
P_{0}=0_{6 \times 6}, \quad P_{1}=\left[\begin{array}{llllll}
0 & 0 & 0 & 1 & 0 & 0
\end{array}\right]^{T}, \quad P_{2}=\left[\begin{array}{llllll}
0 & 0 & 0 & 0 & 1 & 0
\end{array}\right]^{T}, \\
P_{3}=\left[\begin{array}{llllll}
0 & 0 & 0 & 0 & 0 & 1
\end{array}\right]^{T}, \quad P_{4}=\left[\begin{array}{llllll}
0 & 0 & 0 & 0 & 0 & 1
\end{array}\right]^{T}, \quad P_{5}=\left[\begin{array}{llllll}
0 & 0 & 0 & 0 & 0 & 1
\end{array}\right]^{T}, \\
P_{6}=\left[\begin{array}{llllll}
0 & 0 & 0 & 0 & 1 & 0
\end{array}\right]^{T}, \quad P_{7}=\left[\begin{array}{llllll}
0 & 0 & 0 & 1 & 0 & 0
\end{array}\right]^{T}, \quad P_{8}=\left[\begin{array}{cc}
P_{8 t} & 0 \\
0 & P_{8 r}
\end{array}\right]
\end{gathered}
$$

where

$$
P_{8 t}=\left[\begin{array}{ccc}
1 & 0 & 0 \\
0 & 1 & 0 \\
0 & 0 & 1
\end{array}\right] \quad P_{8 r}=\left[\begin{array}{ccc}
1 & 0 & -\sin \left(\delta_{\beta}\right) \\
0 & \cos \left(\delta_{\alpha}\right) & \cos \left(\delta_{\alpha}\right) \sin \left(\delta_{\beta}\right) \\
0 & -\sin \left(\delta_{\alpha}\right) & \cos \left(\delta_{\alpha}\right) \cos \left(\delta_{\beta}\right)
\end{array}\right] .
$$

Similarly, the $P_{i}$ are used to relate the relative generalized velocities to the rates of the joint variables by [5]

$$
\hat{\mathcal{V}}_{0}=\mathcal{V}_{0}, \quad \hat{\mathcal{V}}_{i}=P_{i} \dot{\theta}_{i}, i \in\{1, \ldots, 7\}, \quad \hat{\mathcal{V}}_{8}=P_{8} \dot{\delta}
$$

The first term on the left represents the absolute generalized base velocity $\mathcal{V}_{0}$. In the next section we will formally set $\mathcal{V}_{0}=0$ to render the base of the manipulator stationary. The next seven entries represent the rates of rotation of the seven actuated joints, i.e. $\dot{\theta}_{i}$ is the rate of rotation of frame $F_{i}$ about $O_{i}$ with respect to frames $F_{\bar{i}}$ and $F_{i-1}$. This rate is then translated to the correct rotation axis by $P_{i}$, such that $\hat{\mathcal{V}}_{i}$ evolves in the required direction. Lastly, $\dot{\delta}_{i}$ represents the six distinct rates for the 6-DOF FMS associated with the remaining body.

\section{Chain of Bodies}

In this section we link the set of nine bodies by generating 'system-level' versions of the various quantities already defined for a single body. In the sequel we will denote 'system' matrices with a subscript $*$. We now form the following system generalized matrices from quantities described in the previous section:

$$
\begin{array}{cc}
\mathcal{V}_{*}=\operatorname{col}\left\{\mathcal{V}_{0}, \ldots, \mathcal{V}_{8}\right\}, & \mathcal{V}_{*} \in \mathbb{R}^{6(9)}, \\
\hat{\mathcal{V}}_{*}=\operatorname{col}\left\{\hat{\mathcal{V}}_{0}, \ldots, \hat{\mathcal{V}}_{8}\right\}, & \hat{\mathcal{V}}_{*} \in \mathbb{R}^{6(9)}, \\
f_{T *}=\operatorname{col}\left\{f_{0 T}, \ldots, f_{8 T}\right\}, & f_{T *} \in \mathbb{R}^{6(9)}, \\
f_{I *}=\operatorname{col}\left\{f_{0 I}, \ldots, f_{8 I}\right\}, & f_{I *} \in \mathbb{R}^{6(9)},
\end{array}
$$




$$
\begin{aligned}
& P_{*}=\left[\begin{array}{ccccc}
0 & 0 & \ldots & \ldots & 0 \\
P 1 & 0 & \ldots & \ldots & \vdots \\
0 & P 2 & \ldots & \ldots & \vdots \\
\vdots & \vdots & \ddots & 0 & \vdots \\
\vdots & \vdots & 0 & P 7 & 0 \\
0 & 0 & 0 & 0 & P 8
\end{array}\right], \quad P_{*} \in \mathbb{R}^{6(9) \times 13} \\
& \hat{f}_{*}=\operatorname{col}\left\{\hat{f}_{0}, \ldots, \hat{f}_{8}\right\}=-P_{*} f_{c *}-Q_{*} f_{o *}, \quad \hat{f}_{*} \in \mathbb{R}^{6(9)} . \\
& M_{*}=\left[\begin{array}{cccc}
M_{0} & 0 & \cdots & 0 \\
0 & M_{1} & \ldots & 0 \\
\vdots & \vdots & \ddots & 0 \\
0 & 0 & 0 & M_{8}
\end{array}\right], \quad M_{*} \in \mathbb{R}^{6(9) \times 6(9)} \\
& T_{*}=\left[\begin{array}{cccccc}
I_{6} & 0 & 0 & \ldots & 0 & 0 \\
T_{0}^{1} & I_{6} & 0 & \ldots & 0 & 0 \\
T_{0}^{2} & T_{1}^{2} & I_{6} & \ldots & 0 & 0 \\
\vdots & \vdots & \ddots & \ddots & \vdots & \vdots \\
T_{0}^{7} & T_{1}^{7} & T_{2}^{7} & \ddots & I_{6} & 0 \\
T_{0}^{8} & T_{1}^{8} & T_{2}^{8} & \ldots & T_{7}^{8} & I_{6}
\end{array}\right], \quad T_{*} \in \mathbb{R}^{6(9) \times 6(9)} \\
& G_{*}=\left[\begin{array}{cccccc}
I_{6} & 0 & 0 & \ldots & 0 & 0 \\
0 & I_{6} & 0 & \ldots & 0 & 0 \\
0 & 0 & I_{6} & \ldots & 0 & 0 \\
\vdots & \vdots & \ddots & \ddots & \vdots & \vdots \\
0 & 0 & 0 & \ddots & I_{6} & 0 \\
0 & 0 & 0 & \ldots & 0 & G_{8,8}
\end{array}\right], \quad G_{*} \in \mathbb{R}^{6(9) \times 6(9)},
\end{aligned}
$$

where $I_{6}$ is the identity matrix of dimension 6 . 
With these new definitions we assemble the following equations of interest [5]:

$$
\begin{array}{r}
M_{*} \dot{\mathcal{V}}_{*}=f_{T *}+f_{I *} \\
\mathcal{V}_{*}=T_{*} G_{*} \hat{\mathcal{V}}_{*} \\
f_{T *}=-T_{*}^{-T} G_{*}^{-T} \hat{f}_{*}+f_{* e x t}
\end{array}
$$

Equation (7.18) now represents the system dynamical equation consisting of nine rows. Equation (7.19) is essentially the matrix form of (7.4), and depicts the relationship between the system generalized velocity $\mathcal{V}_{*}$ and system relative generalized velocity $\hat{\mathcal{V}}_{*}$. In $(7.20)$, the force $f_{T *}$ is decomposed into two parts: the system generalized interbody force $\hat{f}_{*}$, and the system generalized external force $f_{* e x t}$, where $f_{* e x t} \in \mathbb{R}^{6(9)}$.

In (7.18), the term $\dot{\mathcal{V}}_{*}$ represents absolute accelerations. The next step of the derivation is to instead express the system dynamics in terms of relative accelerations. To that end we manipulate (7.18)-(7.20) as follows [5]: First, substitute (7.20) and (7.19) into (7.18); second, apply the product rule to $\frac{\partial}{\partial t}\left(T_{*} G_{*} \hat{\mathcal{V}}_{*}\right)$; third, left-multiply both sides by $G_{*}^{T} T_{*}^{T}$. After completing these steps and reorganizing the terms we obtain

$$
\left(G_{*}^{T} T_{*}^{T} M_{*} T_{*} G_{*}\right) \dot{\dot{\mathcal{V}}_{*}}=-\hat{f}_{*}+G_{*}^{T} T_{*}^{T} f_{* e x t}+G_{*}^{T} T_{*}^{T}\left(f_{I *}-M_{*}\left(\dot{T}_{*} G_{*}+T_{*} \dot{G}_{*}\right) \hat{\mathcal{V}}_{*}\right)
$$

Let $f_{N *} \in \mathbb{R}^{6(9)}$ be a vector representing non-linear terms above such that $f_{N *}:=G_{*}^{T} T_{*}^{T}\left(f_{I *}-\right.$ $\left.M_{*}\left(\dot{T}_{*} G_{*}+T_{*} \dot{G}_{*}\right) \hat{\mathcal{V}}_{*}\right)$. The two components of the non-linear term manifest from the evolution of the manipulator global configuration, $\left(\dot{T}_{*}, \dot{G}_{*}\right)$-terms, and from centrifugal and Coriolis forces that result from the product of the angular and/or linear velocities of two bodies, $\left(f_{I *}\right)$-term. We remark that the so-called rate-linear motion expressions for a serial arrangement of rigid bodies are recouped when $f_{N *}$ is dropped from (7.21).

The next step is to introduce the joint variables $\tilde{\theta}, \delta$ into the model described in (7.21). First, define the system-level version of (7.8) using (7.10) and (7.13) [5]

$$
\hat{\mathcal{V}}_{*}=P_{*} \dot{\tilde{q}}
$$


Equation (7.22) constrains the generalized relative velocity to the degrees of freedom specified in $P_{*}$, while the magnitude of the velocity is dictated by the rate variables of $\dot{\tilde{q}}$. We remark that the definition of $P_{*}$ yields $\hat{\mathcal{V}}_{0}=\mathcal{V}_{0}=0$, such that the base of the manipulator is stationary. Second, we perform an intermediate step by substituting (7.22) and (7.14) into (7.21), and left-multiplying both sides by $P_{*}^{T}$

$$
\left(P_{*}^{T} G_{*}^{T} T_{*}^{T} M_{*} T_{*} G_{*}\right)\left(P_{*} \dot{\tilde{q}}\right)=P_{*}^{T} P_{*} f_{c *}+P_{*}^{T} Q_{*} f_{o *}+P_{*}^{T} G_{*}^{T} T_{*}^{T} f_{* e x t}+P_{*}^{T} f_{N *} .
$$

To simplify this equation we observe that the terms $P_{*}^{T} P_{*} f_{c *}$ and $P_{*}^{T} Q_{*} f_{o *}$ obey the following rules [5]:

1. For $B_{i}, i \in\{1, \ldots, 7\}$ associated with the $\dot{\theta}_{i}$, we have that: $P_{i}^{T} Q_{i}=0_{1 \times 5}, P_{i}^{T} P_{i}=1$.

2. For $B_{8}$ associated with $\dot{\delta}$ we use the small-angle approximation, i.e. $\sin (\tilde{\delta}) \approx 0$ for $\tilde{\delta} \approx 0$, and set $P_{8}^{T} P_{8}:=I_{6}$, the identity matrix of dimension 6 . Moreover, $P_{8}^{T} Q_{8}=\mathbf{0}$ since $Q_{8}=\mathbf{0}$.

3. As a result we have $P_{*}^{T} P_{*} f_{c *}:=f_{c *}, f_{c *} \in \mathbb{R}^{13}$, and $P_{*}^{T} Q_{*} f_{o *}:=\mathbf{0}$.

The last steps to incorporating the joint variables are to apply the product rule to $\left(P_{*} \dot{\tilde{q}}\right)$, rearrange all the terms, and use the notation $T_{P}:=T_{*} G_{*} P_{*}$ to produce

$$
\left(T_{P}^{T} M_{*} T_{P}\right) \ddot{\tilde{q}}=f_{c *}+T_{P}^{T} f_{* e x t}+f_{n o n},
$$

where $f_{\text {non }}=P_{*}^{T} f_{N *}-T_{P}^{T} M_{*} T_{*} G_{*} \dot{P_{*}} \dot{\tilde{q}}$. The term $f_{c *}$ represents the generalized control inputs; in other words the driving torques generated by some feedback law are applied here. The term $T_{P}^{T} f_{* e x t}$ maps the effects of the generalized external forces. Lastly, $f_{\text {non }}$ completely captures the non-linear dynamics arising from configuration changes and inertial forces. Herein we eliminate the non-linear term to obtain a rate-linear dynamical approximation. Effectively, the manipulator operates at such low velocities that the non-linear inertial terms are rendered negligible [5]; additional requirements for the approximation may be found in [39].

The last step in our derivation of the nominal manipulator model is to specify the external force $f_{* e x t}$. Recall from Chapter 4 that the only external force acting on the system is the 
environment force. Let $F_{e}^{8} \in \mathbb{R}^{6}$ be the environment force that acts on the last body of the SPDM defined in $F_{8}$. Then $f_{* e x t}$ is defined as

$$
f_{* e x t}:=\left[\begin{array}{c}
0_{6(8) \times 1} \\
F_{e}^{8}
\end{array}\right]=\left[\begin{array}{c}
0_{6(8) \times 6} \\
I_{6}
\end{array}\right] F_{e}^{8}=W^{T} F_{e}^{8}, \quad W \in \mathbb{R}^{6 \times 54}
$$

Substituting (7.25) into (7.24) generates

$$
\left(T_{P}^{T} M_{*} T_{P}\right) \ddot{\tilde{q}}=f_{c *}+T_{P}^{T} W^{T} F_{e}^{8}=f_{c *}+\left(W T_{P}\right)^{T} F_{e}^{8} .
$$

Comparison of (7.26) to the standard manipulator dynamical equation (4.7) indicates that $W T_{P}$ is equivalent to the $(6 \times n)$ Jacobian, $J$. As a result, let $\tilde{J}^{8} \in \mathbb{R}^{6 \times 13}$ be the Jacobian where $\tilde{J}^{8}:=W T_{P} ;$ the superscript 8 means that the Jacobian is expressed with respect to frame $F_{8}$, the FMS frame. This follows from the fact that $W$ selects the last row of $T_{P}$, in which the interbody transformations $T_{i}^{8}$ transform the constraint matrices $P_{i}$ to frame $F_{8}$ :

$$
\tilde{J}^{8}=W T_{P}=\left[\begin{array}{lllll}
T_{1}^{8} P_{1} & T_{2}^{8} P_{2} & \cdots & T_{7}^{8} P_{7} & G_{8,8} P_{8}
\end{array}\right]
$$

Additionally, $\tilde{J}^{8}$ may be subdivided into Jacobians concerning the joints $\theta_{i}$ and $\delta_{i}$ respectively:

$$
\tilde{J}^{8}=\left[\begin{array}{ll}
\tilde{J}_{\theta}^{8} & \tilde{J}_{\delta}^{8}
\end{array}\right], \quad \tilde{J}_{\theta}^{8} \in \mathbb{R}^{6 \times 7}, \quad \tilde{J}_{\delta}^{8} \in \mathbb{R}^{6 \times 6} .
$$

We now present the final dynamical equation after a slight revision of the notation of (7.26)

$$
\tilde{M}(\tilde{q}) \ddot{\tilde{q}}=\tilde{\tau}+\left(\tilde{J}^{8}\right)^{T} F_{e}^{8},
$$

where $\tilde{q} \in \mathbb{R}^{13}, \tilde{M}(\tilde{q}) \in \mathbb{R}^{13 \times 13}, \tilde{\tau} \in \mathbb{R}^{13}$. Notice that the inertia matrix $\tilde{M}(\tilde{q})$ is configuration dependent, meaning that it changes as the joint vector $\tilde{q}$ evolves. Moreover, the inertia matrix is symmetric and positive definite. Equation (7.29) represents the nominal manipulator model for the SPDM sought in this section. A more detailed and applicable model is pursued in the next section. 


\subsubsection{Augmented Model}

In this section additional terms will be added to the nominal SPDM model representing joint flexibility and structural damping, and a system simplification will be made to remove the dynamics of joints unused for the interaction task.

Consider the SPDM dynamics described by

$$
\tilde{M}(\tilde{q}) \ddot{\tilde{q}}=\left[\begin{array}{c}
\tilde{\tau}_{1} \\
\tilde{\tau}_{2}
\end{array}\right]+\left(\tilde{J}^{8}\right)^{T} F_{e}^{8},
$$

where the vector $\tilde{\tau}$ is decomposed into components $\tilde{\tau}_{1} \in \mathbb{R}^{7}$ and $\tilde{\tau}_{2} \in \mathbb{R}^{6}$, the controls on the joints $\tilde{\theta}$ and $\delta$ respectively. We begin with a discussion of joint flexibility related to the actuation of each joint. First, each actuated joint $\theta_{i}$ of the SPDM is driven by a DC motor whose dynamics are described by [5]

$$
J_{m} \ddot{\psi}+K_{t} K_{b} \dot{\psi} / R_{0}=K_{t} V / R_{0}-\tilde{\tau}_{1} / N
$$

where $\psi \in \mathbb{R}^{7}$ is a vector of motor angles, $V$ is a vector of input voltages, $J_{m}$ is the motor inertia, $K_{t}$ and $K_{b}$ are motor torque and back-EMF constants respectively, $R_{0}$ is the rotor winding resistance, and $N$ represents the gearbox ratio from the input gear to the output gear. The gearbox represents the mechanism by which torques produced by the actuator motors are transmitted to the joints to induce motion for each link of the SPDM. Specifically, viewed from the output side, a difference between the angle of the motor-side of the gearbox input, denoted $N^{-1} \psi$, and the angle of the manipulator joints of the gearbox output, denoted $\tilde{\theta}$, generates a torque through a torsional spring with torsional stiffness $K_{\tilde{\theta}}$. In this manner the inertias of the motors and links are coupled. Note that in (7.31) friction terms for the motors are neglected, but they are detailed in [5]. Thus, a simplified linear model of the torque transmitted to the joints from the gearbox is

$$
\tilde{\tau}_{1}=K_{\tilde{\theta}}\left(\frac{\psi}{N}-\tilde{\theta}\right)
$$

Second, the SPDM is equipped with a wrist-mounted, 6-DOF device that detects forces in, and moments about, the three Cartesian directions. The corresponding compressions and twists 
are represented in the system evolution by the $\delta_{i}$ joints. The FMS joints are not driven by motors, rather the stiffness of the joints generates a torque upon displacement from zero, which is defined as

$$
\tilde{\tau}_{2}:=K_{\delta}(0-\delta)
$$

where $K_{\delta} \in \mathbb{R}^{6 \times 6}$ is a diagonal matrix consisting of the joint stiffness terms of the $\delta_{i}$. Substitution of Equations (7.32)-(7.33) into (7.30) and (7.31), and with rearrangement of terms, produces the coupled dynamics

$$
\begin{array}{r}
\tilde{M}(\tilde{q}) \ddot{\tilde{q}}=\left[\begin{array}{c}
K_{\theta}\left(N^{-1} \psi-\tilde{\theta}\right) \\
K_{\delta}(0-\delta)
\end{array}\right]+\left(\tilde{J}^{8}\right)^{T} F_{e}^{8} \\
J_{m} \ddot{\psi}+K_{t} K_{b} \dot{\psi} / R_{0}+N^{-1} K\left(N^{-1} \psi\right)=K_{t} V / R_{0}+N^{-1} K_{\tilde{\theta}} \tilde{\theta} .
\end{array}
$$

We remark that in the development to follow the motor dynamics are removed; rather than computing input voltages for the motors in order to produce a rotation of the motor shaft and transmit a torque on each joint through the gearbox, instead input torques to the joints are directly assigned by our control laws. Thus, in our development (7.34b) is not used, and $\tilde{\tau}_{1}$ will adopt a new form to be defined. However, the expression for $\tilde{\tau}_{2}$ is retained as this term describes the inherent forces acting on the FMS joints, which cannot be directly actuated.

The second step of this section is to reduce the system order with respect to the number of joints that will be used. We recognize from Chapters 4 and 5 that the interaction task only requires the three pitch joints $\theta_{3}, \theta_{4}$, and $\theta_{5}$, as well as the $\delta_{i}$. In other words, the remaining four joints are to be locked, rendering them unactuated and stationary during the maneouver. This behaviour may be imposed on the system equations by applying a transformation that removes the unneeded rate variables by setting them to 0 [5]. To that end let $q \in \mathbb{R}^{9}$ be the new generalized coordinate vector, and $\theta \in \mathbb{R}^{3}$ the subset of joint variables defined as

$$
q:=\left[\begin{array}{ll}
\theta & \delta
\end{array}\right]^{T}, \quad \theta:=\left[\begin{array}{lll}
\theta_{3} & \theta_{4} & \theta_{5}
\end{array}\right]^{T} .
$$


Then one may use the transformation $R$ to relate the joint rates $\dot{q}$ and $\dot{\tilde{q}}$ as follows

$$
\dot{\tilde{q}}=\left[\begin{array}{c}
0 \\
0 \\
\dot{\theta}_{3} \\
\dot{\theta}_{4} \\
\dot{\theta}_{5} \\
0 \\
0 \\
\dot{\delta}
\end{array}\right]=\left[\begin{array}{cc}
0 & 0 \\
I_{3} & 0 \\
0 & 0 \\
0 & I_{6}
\end{array}\right]\left[\begin{array}{c}
\dot{\theta}_{3} \\
\dot{\theta}_{4} \\
\dot{\theta}_{5} \\
\dot{\delta}
\end{array}\right]=R\left[\begin{array}{c}
\dot{\theta} \\
\dot{\delta}
\end{array}\right]=R \dot{q}, \quad R \in \mathbb{R}^{13 \times 9}
$$

where $I_{3}, I_{6}$ are the identity matrix with dimension 3 and 6 respectively. One can integrate the effects of $R$ into the dynamic equation (7.30) by referring back to (7.22), and substituting $\dot{\tilde{q}}=R \dot{q}$. Performing the subsequent steps of the model derivation will ultimately lead to the dynamic equation

$$
\left(R^{T} \tilde{M} R\right) \ddot{q}=R^{T} \tilde{\tau}+R^{T}\left(\tilde{J}^{8}\right)^{T} F_{e}^{8} .
$$

The result is that the state size has been reduced from 13 to 9 . We refine the notation as follows. Let $M=R^{T} \tilde{M} R$ and $J^{8}=\tilde{J}^{8} R$. Moreover, let $\tau \in \mathbb{R}^{9}$ be the control vector defined as

$$
\tau:=\left[\begin{array}{l}
\tau_{1} \\
\tau_{2}
\end{array}\right]=R^{T}\left[\begin{array}{c}
\tilde{\tau}_{1} \\
\tilde{\tau}_{2}
\end{array}\right] .
$$

By definition of $R$ we have that $\tau_{2}=\tilde{\tau}_{2}$. Rewriting (7.36) with this new notation we obtain

$$
M \ddot{q}=\left[\begin{array}{c}
\tau_{1} \\
\tau_{2}
\end{array}\right]+\left(J^{8}\right)^{T} F_{e}^{8},
$$

where $M \in \mathbb{R}^{9 \times 9}, J^{8} \in \mathbb{R}^{6 \times 9}$. Finally, let $K=\operatorname{diag}\left\{0, K_{\delta}\right\} \in \mathbb{R}^{9 \times 9}$ be the stiffness matrix associated with the joints $q$. Then, recalling the expression for $\tau_{2}$ in (7.33), we can rewrite 
equation (7.37) as

$$
M \ddot{q}+K q=\left[\begin{array}{c}
\tau_{1} \\
0
\end{array}\right]+\left(J^{8}\right)^{T} F_{e}^{8} .
$$

The last system modification is the addition of a structural damping term represented as $D \dot{q}$, where $D$ is the configuration-dependent damping matrix multiplied by the joint-rate vector $\dot{q}$. The purpose of generating $D$ is to complete the definition of a second order flexible system

$$
M \ddot{q}+D \dot{q}+K q=\tau(u)
$$

given linear, symmetric matrices $M$ and $K$ with state vector $q$. This mathematical tool was used in the modelling of the SPDM in [5], which represents an application of the concept as it is presented in [40]. We now present the method for generating $D$ from system matrices $M$ and $K$. The procedure involves a transformation to a modal system where the notation $\xi$ denotes the modal coordinates, $z$ is the positive scalar damping term, and $\Omega$ is the diagonal matrix containing the system modes:

$$
\ddot{\xi}+2 z \Omega \dot{\xi}+\Omega^{2} \xi=f(u) .
$$

Essentially, an expression for the damping matrix $D$ will emerge upon transformation from (7.39) to (7.40). The transformation process requires the determination of a congruent transformation $T$ satisfying

$$
T^{T} M T=I, \quad T^{T} K T=\Omega^{2} .
$$

This formulation represents a simultaneous diagonalization problem on $M$ and $K$ to which the singular value decomposition (SVD) may be applied in succession. The transformation $T$ is computed by first generating some matrix $T^{\prime}$ such that $(T)^{\prime T} M T^{\prime}=I$, an approach called whitening the inertia matrix, then by diagonalizing the resulting matrix $(T)^{\prime T} K T^{\prime}$ with an orthonormal transformation. Briefly, the whole process begins by applying SVD to the inertia matrix, which can be used to generate its square root and inverse square root. A series of change of basis steps are then performed along with an additional SVD application, after which the expression for the damping matrix is found. The process is summarized below. The first 
SVD application decomposes $M$ and generates the following identity

$$
M=U S U^{T}, \Longrightarrow M^{-1 / 2}=U S^{-1 / 2} U^{T}
$$

We then define the new basis $q=M^{-1 / 2} \gamma$ which along with (7.42) is substituted into (7.39). The first congruent transformation is completed by multiplying the result by $M^{-T / 2}$ to yield

$$
\ddot{\gamma}+U S^{-T / 2} U^{T} D U S^{-1 / 2} U^{T} \dot{\gamma}+U S^{-T / 2} U^{T} K U S^{-1 / 2} U^{T} \gamma=M^{-1 / 2} K \tau(u)
$$

Thus the whitening step has been completed. Another change of basis $\gamma=U \eta$ is used along with a pre-multiplication by $U^{T}$ to give

$$
\ddot{\eta}+S^{-T / 2} U^{T} D U S^{-1 / 2} \dot{\eta}+S^{-T / 2} U^{T} K U S^{-1 / 2} \eta=U^{T} M^{-1 / 2} K \tau(u) .
$$

The second SVD is now executed with respect to the $K$ term where

$$
S^{-T / 2} U^{T} K U S^{-1 / 2}=V \Omega^{2} V^{T}
$$

The last change of basis $\xi=V \eta$ is performed, along with substitution and congruent transformation to obtain the final modal coordinate representation:

$$
\ddot{\xi}+V^{T} S^{-T / 2} U^{T} D U S^{-1 / 2} V \dot{\xi}+\Omega^{2} \xi=V^{T} U^{T} M^{-1 / 2} K \tau(u) .
$$

In (7.46) the mode shapes are the eigenvectors associated with the system modes in $\Omega$. From canonical second order systems the $D$ matrix will be such that the second term on the left satisfies $2 z \Omega$ :

$$
V^{T} S^{-T / 2} U^{T} D U S^{-1 / 2} V=2 z \Omega \Longrightarrow D=2 z\left(U S^{-1 / 2} V\right)^{-T} \Omega\left(U S^{-1 / 2} V\right)^{-1}
$$


Since $D$ depends on the configuration-dependent $M$, it must be computed at each step for simulation. Given the sparsity of $K$, the computed matrix $D$ has the form

$$
D=\left[\begin{array}{cc}
0 & 0 \\
0 & D_{\delta}
\end{array}\right], \quad D_{\delta} \in \mathbb{R}^{6 \times 6},
$$

such that the damping matrix is decoupled between the equations of motion for the $\theta_{i}$ and $\delta_{i}$.

The modelling of the SPDM is now complete, with the final dynamical equation expressed below. The methods of Chapters 4 - 6 will be applied to the SPDM described by the dynamics

$$
M \ddot{q}+D \dot{q}=\left[\begin{array}{c}
\tau_{1} \\
-K_{\delta} \delta
\end{array}\right]+\left(J^{8}\right)^{T} F_{e}^{8} .
$$

We conclude this section with a brief description of the physical structure of the SPDM and the arrangement of frames. Figures 7.3-7.4 depict the SPDM from the top and side respectively. The figures are annotated with the frames $F_{i}, i=0,1,2, \ldots, 8, \overline{9}$, where $F_{0}$ is the base joint, $F_{1}-F_{7}$ are labelled in association with an actuated joint $\theta_{i}, F_{8}$ is the frame associated with the FMS, and $F_{\overline{9}}$ is the tip. Note that the orientations of $F_{i}$ and $F_{i \overline{+} 1}$ are related by a simple identity matrix. In the figures the SPDM is arranged in what is called the zero-config, where each joint equals $0^{\circ}$; such a physical configuration is not ideal in practice, but is useful for defining lengths, and represents a full outward extension from the base along the $x_{0}$ axis. In Table 7.2 the components of each interbody vector $r_{i, i \overline{+}}$ are provided in terms of the physical components that collectively make up the SPDM's construction. Finally, the figures are annotated with various lengths; these lengths may be described by the measurements given in the table, and are defined in the next section. 


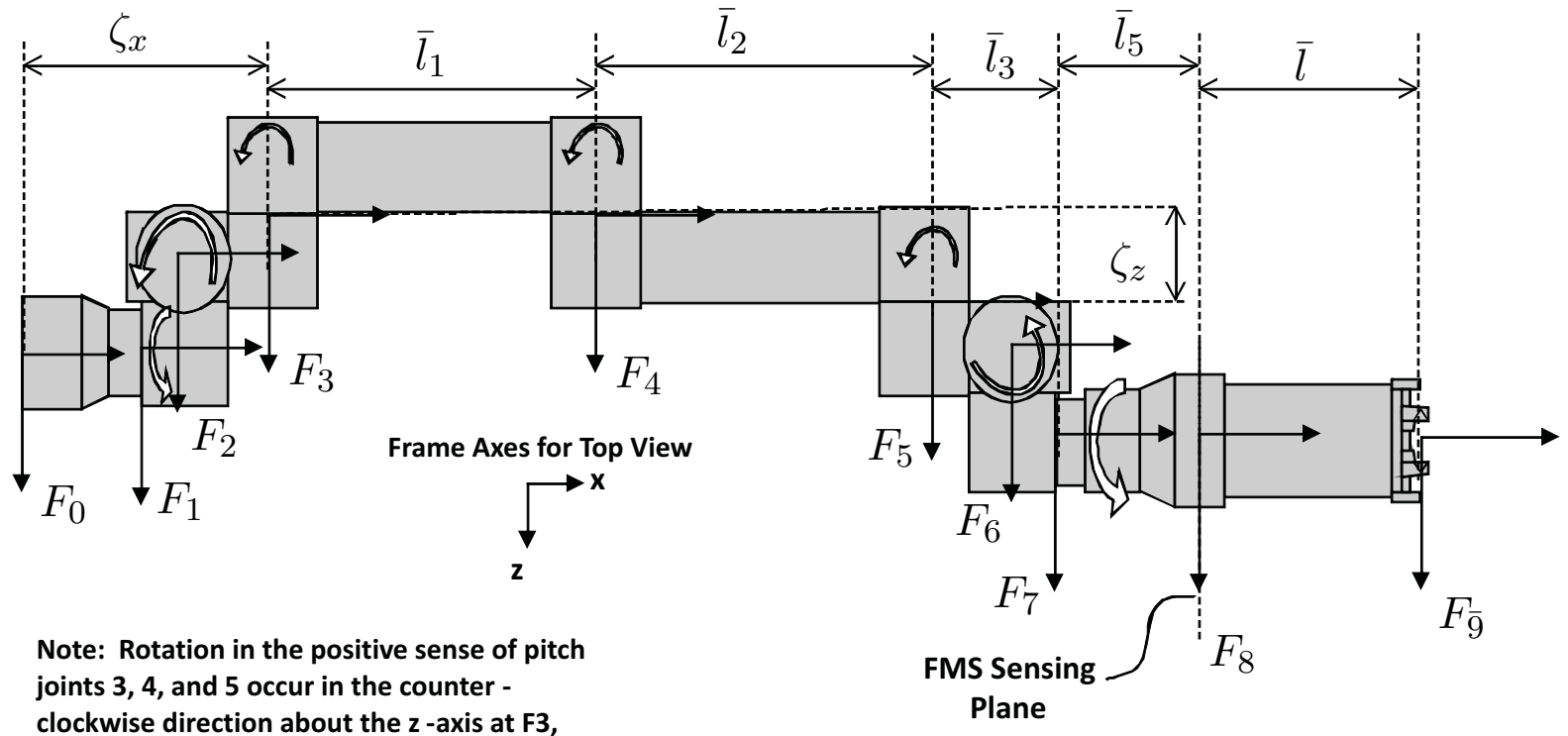

Figure 7.3: SPDM Top View [38].

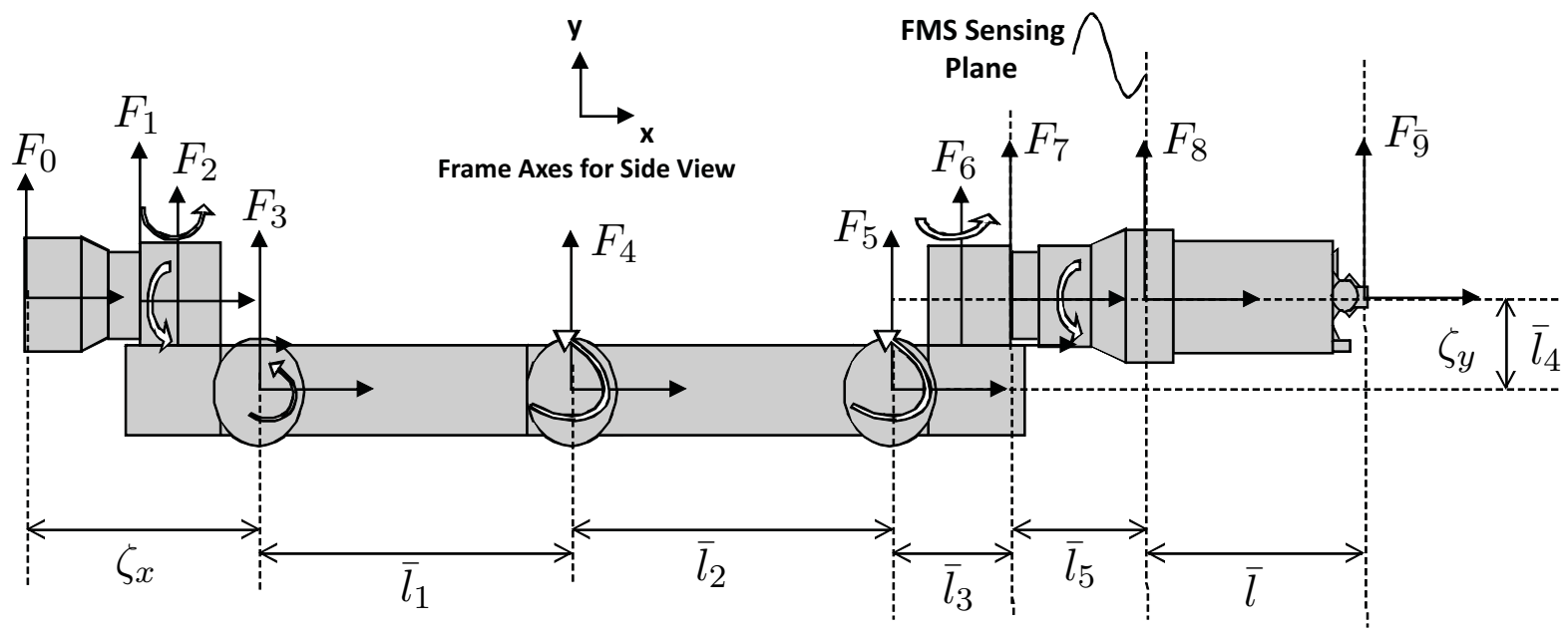

Figure 7.4: SPDM Side View [38].

\subsection{System Modelling with SPDM}

With the dynamical model of the SPDM in hand, the goal now is to frame the SPDM architecture in terms of a Two-Mass system like that of Section 4.2. Thus, the first portion of this section will subdivide the SPDM into a smaller three-link manipulator and second mass connected through a sensing mechanism. The forward and velocity kinematics of the smaller manipulator will be presented, and a feedback linearisation will be derived to transform its dynamics to joint space. Then, as in Section 4.2, those results will be used to generate the 
system models for control design.

Clearly, the reduction of the SPDM dynamics in the previous section by the 'locking' of joints $\theta_{1}, \theta_{2}, \theta_{6}, \theta_{7}$ effectively renders it a three-link planar manipulator followed by the last body beyond the FMS. Figure 7.5 depicts the equivalent manipulator, in a reduced structure, projected to the $x_{3}-y_{3}$ plane of frame $F_{3}$ at joint 3 , where the positive $z_{3}$ axis points out of the page. Notice that by locking $\theta_{1}$ and $\theta_{2}$ at $0^{\circ}$ we ensure that frames $F_{0}$ and $F_{3}$ of the SPDM are completely parallel. In this diagram we consider the portion of the SPDM extending from frame $F_{3}$ to $F_{\overline{8}}$, where $F_{\overline{8}}$ is located at the base of the FMS sensing plate. Note that when $\delta=0$, the frames $F_{\overline{8}}$ and $F_{8}$ coincide directly. In other words, $F_{\overline{8}}$ denotes the end of the three-link manipulator, while $F_{8}$ denotes the beginning of the second mass defined in Chapter 4.

The immediate goal of this section is to obtain the forward and velocity kinematics of the end effector of the reduced manipulator at frame $F_{\overline{8}}$ relative to the base of the full manipulator at $F_{0}$. These expressions are derived with respect to the reduced dynamics (7.49). First, we remark that one can again verify by inspection that the frame placement of the SPDM and this reduced structure both comply with the DH convention; the enumeration of the frames differs slightly, which is of no consequence. Moreover, the locations of the frames $F_{6}$ and $F_{7}$ of Figure 7.5 differ slightly than those of the SPDM model to simplify the derivation; since no joint actuation occurs at these frames there is no adverse impact.

Along a similar vein as Chapter 4 let the variables $\left(x_{e}, y_{e}, z_{e}\right)$ represent the position of frame $F_{\overline{8}}$, and let $\phi$ characterize the orientation of $F_{\overline{8}}$ with respect to $F_{0}$. The expressions for these

Table 7.2: Manipulator Structure

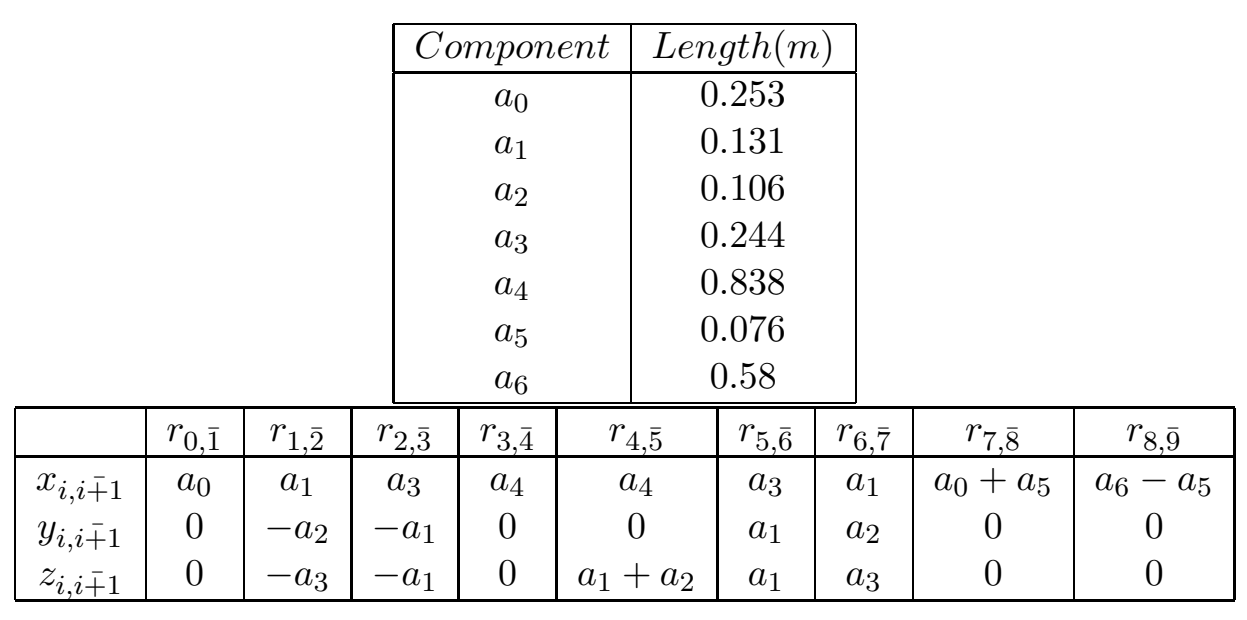




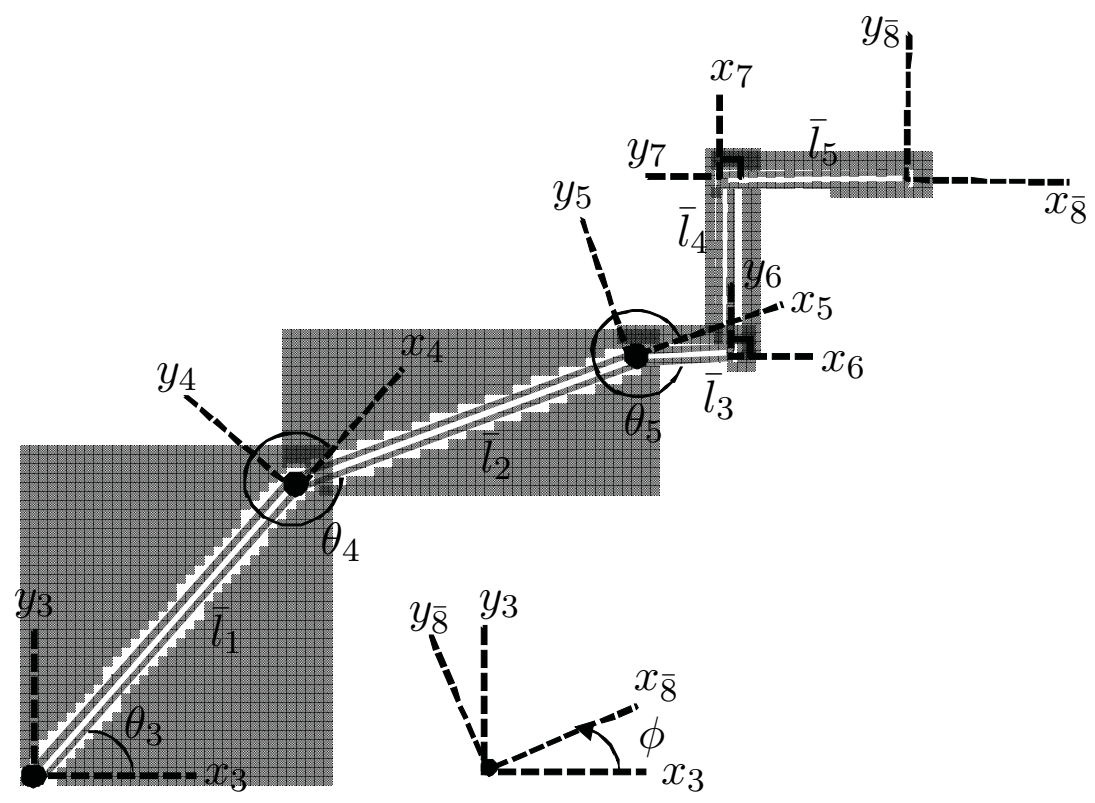

Figure 7.5: Simplified Three-link Manipulator of the SPDM.

quantities predominately depend on the actuated joints, and have been simplified by the fact that four joints are fixed. However, by locking the four excess joints at $0^{\circ}$, we generate an additional constant displacement vector $\zeta \in \mathbb{R}^{3}$ that contributes to the equations: $\zeta_{x}$ and $\zeta_{y}$ are equal to the total displacement from $F_{0}$ to $F_{3}$ listed in Table 7.2 , while $\zeta_{z}$ is the sum of the $z$ components of Table 7.2. Due to the simplicity of the manipulator structure one can obtain by inspection the following forward kinematic equations using the $\bar{l}_{i}$ and $\zeta$ of Figures 7.3-7.5

$$
\begin{aligned}
x_{e} & =\bar{l}_{1} \cos \left(\theta_{3}\right)+\bar{l}_{2} \cos \left(\theta_{3}+\theta_{4}\right)+\bar{l}_{3} \cos \left(\theta_{3}+\theta_{4}+\theta_{5}\right)+\bar{l}_{4} \cos \left(\theta_{3}+\theta_{4}+\theta_{5}+\pi / 2\right)+\bar{l}_{5} \cos \left(\theta_{3}+\theta_{4}+\theta_{5}\right)+\zeta_{x} \\
& =\bar{l}_{1} \cos \left(\theta_{3}\right)+\bar{l}_{2} \cos \left(\theta_{3}+\theta_{4}\right)+\left(\bar{l}_{3}+\bar{l}_{5}\right) \cos \left(\theta_{3}+\theta_{4}+\theta_{5}\right)-\bar{l}_{4} \sin \left(\theta_{3}+\theta_{4}+\theta_{5}\right)+\zeta_{x} \\
y_{e} & =\bar{l}_{1} \sin \left(\theta_{3}\right)+\bar{l}_{2} \sin \left(\theta_{3}+\theta_{4}\right)+\bar{l}_{3} \sin \left(\theta_{3}+\theta_{4}+\theta_{5}\right)+\bar{l}_{4} \sin \left(\theta_{3}+\theta_{4}+\theta_{5}+\pi / 2\right)+\bar{l}_{5} \sin \left(\theta_{3}+\theta_{4}+\theta_{5}\right)+\zeta_{y} \\
& =\bar{l}_{1} \sin \left(\theta_{3}\right)+\bar{l}_{2} \sin \left(\theta_{3}+\theta_{4}\right)+\left(\bar{l}_{3}+\bar{l}_{5}\right) \sin \left(\theta_{3}+\theta_{4}+\theta_{5}\right)+\bar{l}_{4} \cos \left(\theta_{3}+\theta_{4}+\theta_{5}\right)+\zeta_{y} \\
z_{e} & =\zeta_{z} \\
\phi & =\theta_{3}+\theta_{4}+\theta_{5}+\pi / 2-\pi / 2=\theta_{3}+\theta_{4}+\theta_{5}
\end{aligned}
$$

For the particular model under consideration we have $\bar{l}_{1}=\bar{l}_{2}=a_{4}=0.838 \mathrm{~m}, \bar{l}_{3}=a_{3}=$ $0.244 \mathrm{~m}, \bar{l}_{4}=a_{1}+a_{2}=0.237 \mathrm{~m}, \bar{l}_{5}=a_{0}+a_{1}+a_{5}=0.46 \mathrm{~m}, \zeta_{x}=a_{0}+a_{1}+a_{3}=0.628 \mathrm{~m}$, $\zeta_{y}=-a_{1}-a_{2}=-0.237 \mathrm{~m}$, and $\zeta_{z}=a_{1}+a_{2}=0.237 \mathrm{~m}$, where the $a_{i}$ are lengths depicted in Table 7.2. The variables $\left(x_{e}, y_{e}, \phi\right)$ will be called the task space coordinates. 
Next, we are interested in the Jacobian that relates the rates of the actuated joints to the velocities of the task space coordinates $\left(x_{e}, y_{e}, \phi\right)$ of frame $F_{\overline{8}}$, with respect to $F_{0}$. To that end, let $J_{\theta}$ be a reduced $3 \times 3$ Jacobian obtained following the method from Chapter 4 such that

$$
\left[\begin{array}{c}
\dot{x}_{e} \\
\dot{y}_{e} \\
\dot{\phi}
\end{array}\right]=J_{\theta}\left[\begin{array}{c}
\dot{\theta}_{3} \\
\dot{\theta}_{4} \\
\dot{\theta}_{5}
\end{array}\right]
$$

where

$$
J_{\theta}=\left[\begin{array}{ccc}
-\left(\bar{l}_{1} \sin \left(\theta_{3}\right)+b+a\right) & -(b+a) & -a \\
\bar{l}_{1} \cos \left(\theta_{3}\right)+d+c & d+c & c \\
1 & 1 & 1
\end{array}\right]
$$

with

$$
\begin{array}{ll}
a=\left(\bar{l}_{3}+\bar{l}_{5}\right) \sin \left(\theta_{3}+\theta_{4}+\theta_{5}\right)+\bar{l}_{4} \cos \left(\theta_{3}+\theta_{4}+\theta_{5}\right) & b=\bar{l}_{2} \sin \left(\theta_{3}+\theta_{4}\right) \\
c=\left(\bar{l}_{3}+\bar{l}_{5}\right) \cos \left(\theta_{3}+\theta_{4}+\theta_{5}\right)-\bar{l}_{4} \sin \left(\theta_{3}+\theta_{4}+\theta_{5}\right) & d=\bar{l}_{2} \cos \left(\theta_{3}+\theta_{4}\right) .
\end{array}
$$

The Jacobian is invertible except in the singular configurations of the manipulator. We have $\operatorname{det} J_{\theta}=\bar{l}_{1} \bar{l}_{2} \sin \left(\theta_{4}\right)$ so the only singularities occur when $\theta_{4}=0$ or $180^{\circ}$.

\section{Feedback Linearisation}

As discussed in Chapter 4, our approach aims to assign a control to $\tau_{1}$ in order to feedback linearise the reduced manipulator structure of Figure 7.5 into joint space and then into task space. However, because the inertia matrix $M$ is not diagonal, the dynamics (7.49) are coupled in $\ddot{\theta}$ and $\ddot{\delta}$; therefore the transformation to joint space using $\tau_{1}$ is not immediately obvious. The preliminary goal then is to reorganize (7.49) into an equivalent form, from which a suitable control for $\tau_{1}$ may be easily identified. The use of such a $\tau_{1}$ will then generate the closed-loop dynamics

$$
\ddot{\theta}=U_{\theta}
$$

where $U_{\theta} \in \mathbb{R}^{3}$ is a new exogenous input.

To this end we first decompose the inertia matrix $M$ into its constituent parts as per its 
derivation from Section 7.1.1. The reader is reminded of the Jacobian matrices $J^{8}=\left[\begin{array}{lll}J_{\theta}^{8} & J_{\delta}^{8}\end{array}\right]=$ $J^{8} R$ as generated in (7.27), (7.28), and (7.36), and the mass matrix $M_{8}$ associated with Body 8, i.e. the second mass. Moreover, let $M_{R} \in \mathbb{R}^{3 \times 3}$ represent the configuration-dependent inertia matrix of the reduced manipulator just presented. We subdivide $M$ as follows

$$
M=\left[\begin{array}{cc}
M_{11} & M_{12} \\
M_{21} & M_{22}
\end{array}\right], \quad M_{11} \in \mathbb{R}^{3 \times 3}, M_{12} \in \mathbb{R}^{3 \times 6}, M_{21} \mathbb{R}^{6 \times 3}, M_{22} \mathbb{R}^{6 \times 6}
$$

where

$$
M_{11}:=M_{R}+\left(J_{\theta}^{8}\right)^{T} M_{8} J_{\theta}^{8}, \quad M_{12}=M_{21}^{T}:=\left(J_{\theta}^{8}\right)^{T} M_{8} J_{\delta}^{8}, \quad M_{22}:=\left(J_{\delta}^{8}\right)^{T} M_{8} J_{\delta}^{8} .
$$

The reorganization begins by writing out explicitly the dynamical equations for the last six rows of (7.49) associated with the second mass

$$
M_{21} \ddot{\theta}+M_{22} \ddot{\delta}+D_{\delta} \dot{\delta}=-K_{\delta} \delta+F_{e}^{8} .
$$

Then, substitute the expressions for $M_{21}$ and $M_{22}$ and collect terms to obtain

$$
\left(J_{\delta}^{8}\right)^{T} M_{8}\left(J_{\theta}^{8} \ddot{\theta}+J_{\delta}^{8} \ddot{\delta}\right)+D_{\delta} \dot{\delta}=-K_{\delta} \delta+F_{e}^{8} .
$$

Lastly, we multiply both sides by the inverse Jacobian transpose $\left(J_{\delta}^{8}\right)^{-T}$ and rearrange terms to produce

$$
M_{8}\left(J_{\theta}^{8} \ddot{\theta}+J_{\delta}^{8} \ddot{\delta}\right)=-\left(J_{\delta}^{8}\right)^{-T} D_{\delta} \dot{\delta}-\left(J_{\delta}^{8}\right)^{-T} K_{\delta} \delta+F_{e}^{8} .
$$

The above equation will be used in a substitution step shortly. Now write out the dynamical equations for the first three rows of (7.49) associated with the reduced manipulator

$$
M_{11} \ddot{\theta}+M_{12} \ddot{\delta}=\tau_{1}+\left(J_{\theta}^{8}\right)^{T} F_{e}^{8} .
$$

Then, substitute the expressions for $M_{11}$ and $M_{12}$ and collect terms to obtain

$$
M_{R} \ddot{\theta}+\left(J_{\theta}^{8}\right)^{T} M_{8}\left(J_{\theta}^{8} \ddot{\theta}+J_{\delta}^{8} \ddot{\delta}\right)=\tau_{1}+\left(J_{\theta}^{8}\right)^{T} F_{e}^{8} .
$$


Now, substitution of (7.53) into (7.54) yields the desired dynamical equation

$$
M_{R} \ddot{\theta}=\tau_{1}+\left(J_{\theta}^{8}\right)^{T}\left(\left(J_{\delta}^{8}\right)^{-T} K_{\delta} \delta+\left(J_{\delta}^{8}\right)^{-T} D_{\delta} \dot{\delta}\right)
$$

We have now obtained an equation of motion for the simplified three-link manipulator as indicated by the $M_{R} \ddot{\theta}$ term. The last term of the equation is of the standard form $J^{T} F$, where $J$ is the Jacobian $\left(J_{\theta}^{8}\right)$ with respect to $F_{8}$, and $F$ is an external force expressed in task space. Let $F_{s}^{8} \in \mathbb{R}^{6}$ represent this external force, expressed in the FMS frame $F_{8}$ as

$$
F_{s}^{8}=\left(J_{\delta}^{8}\right)^{-T} K_{\delta} \delta+\left(J_{\delta}^{8}\right)^{-T} D_{\delta} \dot{\delta}
$$

We call $F_{s}^{8}$ the sensed force, which is in effect generated by the motion of Body 8 with respect to the reduced manipulator, and is characterized by stiffness and damping terms of the FMS joints $\delta_{i}$. Regarding the definition of the sensed force, we assume in the rest of this thesis that the second term may be neglected in the context of control design for three reasons: i) the structural damping term of the manipulator is assumed to be small, i.e. the scalar damping term z of Section 7.1.2 is small; ii) $D_{\delta}$ cannot be easily reproduced in the context of a feedback law; iii) Damping induces convergence to a true sensed force manifested by $K_{\delta} \delta$, rather than continuous oscillation. Therefore we assume that

$$
F_{s}^{8} \approx\left(J_{\delta}^{8}\right)^{-T} K_{\delta} \delta
$$

Using this assumption we rewrite (7.55) as

$$
M_{R} \ddot{\theta}=\tau_{1}+\left(J_{\theta}^{8}\right)^{T}\left(F_{s}^{8}\right)=\tau_{1}+\left(J_{\theta}^{8}\right)^{T}\left(\left(J_{\delta}^{8}\right)^{-T} K_{\delta} \delta\right) .
$$

From here we set $\tau_{1}$ to be

$$
\tau_{1}=M_{R} U_{\theta}-\left(J_{\theta}^{8}\right)^{T}\left(J_{\delta}^{8}\right)^{-T} K_{\delta} \delta .
$$

Substituting the above control into (7.57) then generates the desired joint space dynamics

$$
\ddot{\theta}=U_{\theta}
$$


Note that the above transformation requires the inverse $\left(J_{\delta}^{8}\right)^{-T}$ to be defined. Recall that $J_{\delta}^{8}=$ $G_{8,8} P_{8}$ as defined in Section 7.1.1 which is singular when $\delta_{\beta}= \pm \frac{\pi}{2}$ or $\delta_{\beta}-\delta_{\alpha}= \pm \frac{\pi}{2}$. We have now completed the simplifications to the original SPDM model. The resulting model contains no actuators, three actuated joints rather than seven, and after a feedback transformation the dynamics either in task space or joint space are linear. With these results in hand we recast the scenario of Section 4.2 in terms of the SPDM.

\section{Two-Mass Modelling}

Now consider the SPDM illustrated in Figure 7.4 consisting of a reduced three-link manipulator whose base is fixed at frame $F_{0}$, and has end effector frame $F_{\overline{8}}$. Attached to the end effector is a second mass $m_{2}$, connected through a set of joints, $\delta$, with stiffness $K_{\delta}$. With respect to the base and in the $x_{0}-y_{0}$ plane, consider $\left(x_{1}, y_{1}, \phi\right)$ as the task space coordinates of the reduced manipulator's end effector, and let $\left(x_{2}, y_{2}\right)$ be the position of the tip of the second mass. A stiff environment is located at $\left(\hat{\rho}_{e}, 0\right)$, which imparts a force on the end of the second mass.

The overall manipulator dynamics are described by (7.49) of Section 7.1.2. To aid in the control we obtain the forward kinematics and the Jacobian $J_{\theta}$ for the end effector of the reduced manipulator from (7.50) and (7.52) respectively (replacing $x_{e}, y_{e}$ with $x_{1}, y_{1}$ ). These expressions are given with respect to the base at $F_{0}$. We proceed now to transform the nonlinear dynamics of (7.49) into a linear form with respect to the task space coordinates. First, we perform an initial feedback transformation to directly control the three joint dynamics. Let $U_{\theta} \in \mathbb{R}^{3}$ be a new exogenous input, let $M_{R}$ be the configuration-dependent inertia of the reduced manipulator, let $J_{\theta}^{8}$ be the sub-Jacobian obtained from (7.49), and let $F_{s}^{8}$ be the sensed force from (7.56). Then define the feedback transformation $\tau_{1}=M_{R} U_{\theta}-\left(J_{\theta}^{8}\right)^{T} F_{s}^{8}$. From the discussion of feedback linearisation in the previous section, and the development from (7.54)-(7.59), it can be shown that substitution of $\tau_{1}$ in (7.49) produces the dynamics

$$
\ddot{\theta}=U_{\theta}
$$

In order to define a feedback transformation to task space, consider the following. Let $F_{s}$ be the sensed force expressed with respect to the base frame $F_{0}$ (obtained by rotating $F_{s}^{8}$ by 
the rotation matrix $\left.C_{8}^{0}\right)$. Note that $(7.60)$ and the task space coordinates have only three DOF, while the sensed force, environment force, and dynamics of the second mass have 6 DOF. The DOF in common are the translational components in the $x_{0}$ and $y_{0}$ plane of the base frame, and the rotational component about the $z_{0}$ axis. For the DOF of $\delta$ not associated with a task space coordinates it is assumed that the inherent joint stiffness and structural damping will render them zero, i.e. $\delta_{z} \approx 0, \delta_{\gamma} \approx 0^{\circ}$, and $\delta_{\alpha} \approx 0^{\circ}$. As a result, the FMS frame $F_{8}$ lies in the same plane as $F_{0}$, and the two will be strictly parallel when $\delta_{\beta}=0^{\circ}$. Therefore, let $F_{s_{R}} \in \mathbb{R}^{3}$ be the vector of forces consisting of the first, second, and sixth rows of $F_{s}$, i.e.

$$
F_{s_{R}}=\left[\begin{array}{cccccc}
1 & 0 & 0 & 0 & 0 & 0 \\
0 & 1 & 0 & 0 & 0 & 0 \\
0 & 0 & 0 & 0 & 0 & 1
\end{array}\right] F_{s} .
$$

Then let $m_{1}>0$ be a to-be-determined parameter for the first mass, and $U \in \mathbb{R}^{3}$ a second exogenous input. We now define a second feedback transformation to transform (7.60) to task space

$$
U_{\theta}=J_{\theta}^{-1}(\theta)\left(\frac{1}{m_{1}} U+\frac{1}{m_{1}} F_{s}-\dot{J}_{\theta}\left[\begin{array}{c}
\dot{\theta}_{3} \\
\dot{\theta}_{4} \\
\dot{\theta}_{5}
\end{array}\right]\right) \text {. }
$$

Using the development of (4.13)-(4.15), the transformation (7.61) generates the dynamics

$$
m_{1}\left[\begin{array}{c}
\ddot{x}_{1} \\
\ddot{y}_{1} \\
\ddot{\phi}_{1}
\end{array}\right]=U+F_{s_{R}}
$$

Equation (7.62) yields three uncoupled double integrator systems so that each element of $U$ can be independently assigned to independently control each task space variable of the reduced manipulator's end effector. Recall that these task space coordinates represent only three of the possible six available to define the motion of a rigid body.

Consider also the dynamics of the second mass with inertia matrix $M_{8}$ expressed in terms 
of the joint variables

$$
M_{8} J_{\theta}^{8} \ddot{\theta}+M_{8} J_{\delta}^{8} \ddot{\delta}=-F_{s}^{8}+F_{e}^{8} .
$$

We express the above dynamics in $F_{0}$ as follows. First, let $X_{1}^{8} \in \mathbb{R}^{6}$ be the full vector of task space coordinates of the first mass with respect to $F_{8}$. Using $(4.13)$ we approximate $\ddot{X}_{1}^{8}=J_{\theta}^{8} \ddot{\theta}$. The acceleration vector may be transformed to frame $F_{0}$ using the rotation matrix $C_{0}^{8} \in \mathbb{R}^{6 \times 6}$, such that $\ddot{X}_{1}^{8}=C_{0}^{8} \ddot{X}_{1}$. Therefore, substitute $J_{\theta}^{8} \ddot{\theta}=C_{0}^{8} \ddot{X}_{1}$ into (7.63), and left-multiply by $\left(C_{0}^{8}\right)^{-1}$ so that every term is expressed in $F_{0}$

$$
\left(C_{0}^{8}\right)^{-1} M_{8} C_{0}^{8} \ddot{X}_{1}+\left(C_{0}^{8}\right)^{-1} M_{8} J_{\delta}^{8} \ddot{\delta}=-F_{s}+F_{e} .
$$

Therefore we have derived in effect a two-mass model consisting of (7.62) for the first mass, and (7.64) which encodes the motion of the last body through the FMS dynamics. One of the main differences between this two-mass system and that of Chapter 4 is that the second mass in this system is not a point mass. Inspection of Figures 7.3 and 7.4 indicate that the second mass has length $\bar{l}$. This length enters the system modelling below.

As in Chapter 4 we apply Assumption 4.2 to obtain a single-mass system from the twomass model suitable for control design. First, from assumption $(\mathrm{C} 1)$ the control task is to be performed such that $\phi=\theta_{1}+\theta_{2}+\theta_{3}=0$, that is, the last body and manipulator end effector only move horizontally. Then let $f_{s} \in \mathbb{R}$ be the first component of $F_{s_{R}}$ when $\phi=0^{\circ}$ and $\delta_{\beta}=0^{\circ}$. Recall that $\delta_{x}$ is a measurement of the relative displacement in $x$ at the last joint, which can be related to absolute quantities. Let $\tilde{x}_{2}$ be the $x$-coordinate of the 'beginning' of the last body such that $\delta_{x}=\tilde{x}_{2}-x_{1}$. Then, $f_{s}$ is given by

$$
f_{s}:=k_{s} \delta_{x}=k_{s}\left(\tilde{x}_{2}-x_{1}\right)
$$

Similarly, let $f_{e} \in \mathbb{R}$ be the non-zero component of environment force $F_{e}$, that acts along the $x_{0}$-axis, and let $x_{2}$ be the $x$-coordinate of the tip of the last body with respect to the base. Then, $f_{e}$ is non-zero when the tip of the last body is in contact with the environment located at $\left(\hat{\rho}_{e}, 0\right)$, and is modelled as a virtual spring with spring constant $k_{e}$ attached between the last 
body and the environment:

$$
f_{e}=\left\{\begin{array}{cc}
k_{e}\left(\hat{\rho}_{e}-x_{2}\right) & x_{2} \geq \hat{\rho}_{e} \\
0 & x_{2}<\hat{\rho}_{e} .
\end{array}\right.
$$

Again we assume that $k_{e} \in\left[k_{e m i n}, k_{e m a x}\right]$. Since the last body is considered to be rigid, and its motion is considered to be purely horizontal (from $\phi \approx 0$ ), then the relationship between $x_{2}$ and $\tilde{x}_{2}$ is given by

$$
\tilde{x}_{2}=x_{2}-\bar{l}
$$

where $\bar{l}$ is the length of the last body depicted (from frame $F_{8}$ to the tip) in Figures 7.3-7.4.

Finally, let $u \in \mathbb{R}$ be the first component of $U$. Then, the equations of motion of interest are the $x$-dynamics of $(7.62)$

$$
m_{1} \ddot{x}_{1}=f_{s}+u
$$

The next assumption depends on the relative magnitudes of $m_{1}$ and the inertia of the last body. In particular, let $m_{2}$ be the nominal mass of the last body used in the construction of the inertia matrix $M_{8}$. We proceed as in Chapter 4, where the assumption (C2), which states that $m_{1}>>m_{2}$, allows us to disregard certain dynamics in (7.64). In the case of no contact we use the result in [37] to conclude that the relative acceleration of the two masses is approximately zero such that $\ddot{\delta} \approx 0$; therefore, substitute $\ddot{\delta}=0$ into (7.64). Then, since $\left(C_{0}^{8}\right)^{-1} M_{8} C_{0}^{8} \ddot{X}_{1} \in \mathbb{R}^{6}$ let the notation $g\left(m_{2}, \ddot{X}_{1}\right) \in \mathbb{R}$ denote its first row entry. The term $g\left(m_{2}, \ddot{X}_{1}\right)$ is dominated by the $x$-direction dynamics of the first mass multiplied by the inertia of the second mass $m_{2}$. Finally, if the first row of (7.64) is extracted and the terms rearranged we obtain:

$$
f_{s}=f_{e}-g\left(m_{2}, \ddot{X}_{1}\right)
$$

Next, substitute (7.69) into (7.68), apply $f_{e}=0$ for no contact, and apply $m_{1}>>m_{2}$ such that $m_{1} \ddot{x}_{1}>>g\left(m_{2}, \ddot{X}_{1}\right)$ to obtain the single-mass free motion equation

$$
m_{1} \ddot{x}_{1}=u \text {. }
$$


For the case when there is contact with the environment we proceed in a manner analogous to Chapter 4. First, using assumption (C3) the absolute acceleration of the second mass in the $x$-direction is assumed to be approximately zero. Notice that the left-hand side of (7.64) in effect represents the dynamics of the second mass since it includes a summation of the motion of the end effector of the first mass (first term) and the relative motion of the two masses (second term). By this reasoning we set the left-hand side of (7.64) to be zero, and extract the first row to obtain

$$
0=-f_{s}+f_{e}
$$

Next, substitute (7.65) and (7.66) into the above, replace $\tilde{x}_{2}=x_{2}-\bar{l}$ and solve for $x_{2}$ :

$$
x_{2}=\frac{1}{k_{s}+k_{e}}\left[k_{s}\left(x_{1}+\bar{l}\right)+k_{e} \hat{\rho}_{e}\right]
$$

Substituting (7.65) and (7.71) into (7.68) and simplifying, we obtain

$$
m_{1} \ddot{x}_{1}=\bar{k}\left(\hat{\rho}_{e}-\bar{l}-x_{1}\right)+u
$$

where

$$
\bar{k}:=\frac{k_{s} k_{e}}{k_{s}+k_{e}}
$$

is called the effective spring constant. As in previous chapters, we have $\bar{k} \in\left[\bar{k}_{\text {min }}, \bar{k}_{\text {max }}\right]$.

Finally, derive a model for the dynamics of the sensed force as before. First we substitute (7.71) into (7.65) and simplify to get

$$
f_{s}=\bar{k}\left(\hat{\rho}_{e}-\bar{l}-x_{1}\right) .
$$

Now take the second derivative and substitute the model (7.72) to obtain the second-order force dynamics

$$
m_{1} \ddot{f}_{s}+\bar{k} f_{s}+\bar{k} u=0 .
$$


We retain the previous force controller to stabilize $f_{s}$ to some $f_{s}^{d}<0$

$$
u_{f}=-f_{s}+k_{1}\left(f_{s}-f_{s}^{d}\right)+k_{2} \dot{f}_{s}
$$

where parameters $k_{1}, k_{2}>0$ determine the closed-loop poles.

We can now summarize all the state space models that will be used for control design. Let $\xi=\left(x_{1}, \dot{x}_{1}\right)$. When there is no contact with the environment, the free motion of the first mass is given by

$$
\dot{\xi}=\left[\begin{array}{ll}
0 & 1 \\
0 & 0
\end{array}\right] \xi+\left[\begin{array}{c}
0 \\
\frac{1}{m_{1}}
\end{array}\right] u \text {. }
$$

The output of this model is the position of the tip of last body given by

$$
x_{2}=\xi_{1}+\bar{l} .
$$

When there is contact with the environment, the single mass approximation of the two mass system is

$$
\dot{\xi}=\left[\begin{array}{cc}
0 & 1 \\
0 & 0
\end{array}\right] \xi+\left[\begin{array}{c}
0 \\
\frac{1}{m_{1}}
\end{array}\right] u+\left[\begin{array}{c}
0 \\
\frac{1}{m_{1}}
\end{array}\right] f_{s} .
$$

Based on (7.71), the output of this model is the position of the tip of the last body given by

$$
x_{2}=\frac{1}{k_{s}+k_{e}}\left[k_{s}\left(\xi_{1}+\bar{l}\right)+k_{e} \rho_{e}\right] .
$$

Next, by substituting (7.75) into (7.68), we obtain the state model for the closed-loop force dynamics when there is contact with the environment

$$
\dot{\xi}=\left[\begin{array}{cc}
0 & 1 \\
-\frac{\bar{k} k_{1}}{m_{1}} & -\frac{\bar{k} k_{2}}{m_{1}}
\end{array}\right] \xi+\left[\begin{array}{c}
0 \\
\frac{\bar{k} k_{1} \rho_{f}^{d}}{m_{1}}
\end{array}\right],
$$

where, letting $\rho_{e}=\hat{\rho}_{e}-\bar{l}$ and recalling that $f_{s}^{d}<0$ we define the position corresponding to the contact force $f_{s}^{d}$ as

$$
\rho_{f}^{d}:=\hat{\rho}_{e}-\bar{l}-\frac{f_{s}^{d}}{\bar{k}}=\rho_{e}+\frac{\left|f_{s}^{d}\right|}{\bar{k}}
$$


System (7.76c) with output (7.76d) is the primary model for which a control scheme will be developed. It represents the motion of one large mass with a control input, and an opposing environmental force. Thus, we have reduced our initial problem of controlling a robotic manipulator with a mass at its end effector to controlling a single mass.

Lastly, we extract the dynamics of the remaining task space variables $y_{1}$ and $\phi$ from (7.62). Given the previous discussion, when $\phi$ is regulated to approximately zero we have that the second and third of components of $F_{s_{R}}$ will be approximately zero. Let $u_{y}, u_{\phi} \in \mathbb{R}$ be the second and third components of $U$. Then the remaining task space dynamics are given by the following

$$
m_{1} \ddot{y}_{1}=u_{y}, \quad m_{1} \ddot{\phi}=u_{\phi} .
$$

By construction, the output of the model for $y_{1}$ is $y_{2}$ of the second mass given by

$$
y_{2}=y_{1}
$$




\section{Chapter 8}

\section{Control Design for the SPDM}

In this chapter we perform the control design and simulation of the interaction task for the SPDM, and apply the solution to the bouncing problem of manipulators. In particular, we consider the same logic control specifications of Problem 5.1 and apply the methodology of Chapter 5 to obtain suitable reach controllers that achieve a bumpless transfer from reach to force control. The main difference between the development to follow and that of Chapter 6 is that the SPDM will be used. Consequently, we proceed as in Chapter 6 by first defining the problem parameters relating to the SPDM and then synthesising the reach controllers. Afterwards, a set of simulations are performed to validate the design.

\subsection{Reach Control Design}

As in the previous design we are interested in presenting the logic control specifications in terms of the states $\xi$ of the first mass, for which the model (7.76a)-(7.76b) is available. However, the 'second mass' of the SPDM is not a point mass, so its length must be considered. Another difference from the previous manipulator is that the rest-length of the sensor is zero. Therefore, assuming purely horizontal motion, the new relationship between the logic control specifications for $\xi$ and the states $\left(x_{2}, \dot{x}_{2}\right)$ of the tip of the SPDM is given by

$$
\begin{aligned}
& x_{2}=\xi_{1}+\bar{l} \\
& \dot{x}_{2}=\xi_{2},
\end{aligned}
$$


where $\bar{l}=0.503 \mathrm{~m}$. Similarly, we introduce the following variables to relate the position of the environment to a position of the first mass:

$$
\begin{aligned}
\rho_{\text {emin }} & :=\hat{\rho}_{\text {emin }}-\bar{l} \\
\rho_{\text {emax }} & :=\hat{\rho}_{\text {emax }}-\bar{l} \\
\rho_{e} & :=\hat{\rho}_{e}-\bar{l} .
\end{aligned}
$$

Using these relationships the methodology of Chapter 5 may be applied for the SPDM.

We now consider Problem 5.1 for the SPDM, and choose a new set of nominal problem parameters. The physical dimensions of the SPDM were already given in the previous chapter, while the specific masses and inertias are contained in the SPDM simulator. In the simulator, the mass of the last body is given as $m_{2}=40 \mathrm{~kg}$, and we again choose a large mass ratio $m_{1} / m_{2}=100$ such that $m_{1}=4000 \mathrm{~kg}$. The sensor parameters are given by $r_{s}=0 \mathrm{~m}$, and $k_{s}=2.1 \cdot 10^{7} \mathrm{~N} / \mathrm{m}$, where $k_{s}$ is the stiffness pertaining to joint $\delta_{x}$ in the stiffness matrix $K_{\delta}$. The range of the environment stiffness is selected as $k_{e} \in\left[10^{7}, 5 \cdot 10^{8}\right] \mathrm{N} / \mathrm{m}$, such that the environment is generally stiffer than the sensor. The effective spring constant range is then $\bar{k} \in\left[6.774 \cdot 10^{6}, 2.015 \cdot 10^{7}\right] \mathrm{N} / \mathrm{m}$. We assume that position sensors can track the environment location to within $1 \mathrm{~cm}$, and so we select $\hat{\rho}_{e} \in[3.003,3.013] \mathrm{m}$, similarly $\rho_{e} \in[2.5,2.51] \mathrm{m}$. Position parameter $\rho_{0}$ is chosen such that the tip of the first mass cannot start further back than the position of the shoulder pitch joint, joint 3 ; assuming $\theta_{1}$, and $\theta_{2}$ are zero we set $\rho_{0}=0.628 \mathrm{~m}$. Lastly, since the manipulator travels slowly we assume a maximum velocity in free space of $\mu_{1}=0.3 \mathrm{~m} / \mathrm{s}$. In regards to the force modality the desired contact force is set to $f_{s}^{d}=-300 \mathrm{~N}$. Recall the force controller (7.75), which requires the measurement of the sensed force $f_{s}$ and its derivative $\dot{f}_{s}$. From the previous chapter, the vector of FMS joints are used in the measurement of the sensed force in the FMS frame where

$$
F_{s}^{8}=\left(J_{\delta}^{8}\right)^{-T} K_{\delta} \delta
$$

Computing the time derivative of the sensed force gives

$$
\dot{F}_{s}^{8}=\left(\dot{J}_{\delta}^{8}\right)^{-T} K_{\delta} \delta+\left(J_{\delta}^{8}\right)^{-T} K_{\delta} \dot{\delta}
$$


The term $\left(\dot{J}_{\delta}^{8}\right)^{-T}$ is negligible for the entries of interest, and so only the second term above remains. It is assumed here therefore that adequate measurement of the rate of change of the FMS joints, $\dot{\delta}$, is available to produce the derivative of the sensed force. With this in mind, the force controller is designed to generate a critically-damped response with a natural frequency $\omega=15 \mathrm{rad} / \mathrm{s}$, when $\bar{k}=\bar{k}_{\text {max }}$. The necessary control parameters are $k_{1}=0.0447, k_{2}=0.006$.

Additionally, the structural damping factor $z$, first described in (7.40), must be selected for the computation of the matrix $D_{\delta}$. Notice that the models and problem formulation of Chapters 4-5 do not incorporate structural damping, while the SPDM does. Therefore, we select $z=0.005$, representing extremely light damping of the FMS joints, to accommodate the proposed solution. However, a simulation will be performed using the default value given in the SPDM simulator, $z=0.05$.

Now for the design we proceed in a manner similar to Chapter 6. First, the PD controllers for $y_{1}$ and $\phi$ are to be critically damped with natural frequency $\omega_{y}=2 \mathrm{rad} / \mathrm{s}$ and $\omega_{\phi}=1 \mathrm{rad} / \mathrm{s}$ respectively. Next, we apply Algorithm 5.1 to obtain the specification parameters that complete the logic control specifications. These specifications then define an operational state space $\mathcal{P}$. The first step is to select a suitable switching force $f_{s w}$; for the SPDM we take two factors into consideration. First, the SPDM model generally ignores inertial forces and configuration changes that would induce forces not associated with contact. Second, light structural damping of the FMS joints is present such that oscillations of the sensed force in free space is minimal. As a result, the magnitude of the switching force is chosen to be small, i.e. $f_{s w}=-10 \mathrm{~N}$. Next in the algorithm we compute $\bar{\mu}_{s w}=2\left(\left|f_{s}^{d}\right|-\left|f_{s w}\right|\right) \frac{k_{2}}{m_{1}}=0.863 \mathrm{~mm} / \mathrm{s}$, and select $\mu_{s w}=0.8 \mathrm{~mm} / \mathrm{s}$ $<\bar{\mu}_{s w}$. In Step 3 we select $\rho_{2}=2.66 \mathrm{~m}>2.51+\frac{10}{\bar{k}_{\text {min }}}$. Finally we select $\mu_{2}=0.9 \mathrm{~mm} / \mathrm{s}>\mu_{s w}$, compute $\rho_{1}=2.48 \mathrm{~m}$, and select $\rho_{3}=2.1 \mathrm{~m}<\rho_{1}$. This set of nominal parameters is compiled into Table 8.1 for quick reference. The regions $\mathcal{P}^{j}, j=1, \ldots, 5$ are then given by:

$$
\begin{array}{ccc}
\mathcal{P}^{1}: & 0.628 \leq \xi_{1} \leq 2.1 & 0 \leq \xi_{2} \leq 0.3 \\
\mathcal{P}^{2}: & 2.1 \leq \xi_{1} \leq 2.48 & 0 \leq \xi_{2} \leq 1.953-0.7871 \xi_{1} \\
\mathcal{P}^{3}: & 2.48 \leq \xi_{1} \leq 2.66 & 0 \leq \xi_{2} \leq 0.005\left(2.66-\xi_{1}\right) \\
\mathcal{P}^{4}: & 2.48 \leq \xi_{1} \leq 2.66 & -0.3 \leq \xi_{2} \leq 0 \\
\mathcal{P}^{5}: & 2.1 \leq \xi_{1} \leq 2.48 & -0.789\left(\xi_{1}-2.1\right) \leq \xi_{2} \leq 0
\end{array}
$$


Reach control design is then performed using (7.76a) for no contact. As in Chapter 6 we calculate $\mathcal{O}=\left\{\xi \mid \xi_{2}=0\right\}$, and triangulate $\mathcal{P}$ as $\mathcal{T}=\left\{\mathcal{S}^{1}, \ldots, \mathcal{S}^{10}\right\}$ as shown in Figure 6.1. The vertices of this triangulation are given as $\mathcal{P}: v_{0}=(0.628,0.3), v_{1}=(0.628,0), v_{2}=(11.4,0.3)$, $v_{3}=(11.4,0) v_{4}=(2.1,0.3), v_{5}=(2.1,0), v_{6}=(2.48,0.0009), v_{7}=(2.48,0), v_{8}=(2.66,0)$, $v_{9}=(2.48,-0.3)$, and $v_{10}=(2.66,-0.3)$. The desired behaviour under reach control is similar to that of the previous design. In particular, Figures 5.2 and 6.1 depict the exit facets of each $\mathcal{P}^{j}$ and desired closed-loop velocities at the vertices to be achieved. Therefore, we select the controls at the vertices using the method described in Chapter 6. Of note is the selection of control $u_{7}^{3}$ in $\mathcal{S}^{10}$ according to Lemma 5.1:

$$
u_{7}^{3}=15>\frac{\left|f_{s w}\right|\left(\rho_{2}-\rho_{1}\right)}{\rho_{2}-\rho_{\text {emax }}-\frac{\left|f_{s w}\right|}{\bar{k}_{\min }}}=12 .
$$

Here $u_{7}^{3}$ is chosen to be $25 \%$ greater than the minimum, as opposed to $100 \%$ in the previous design. After selecting the vertex controls the following reach controllers were synthesised for use with the SPDM.

Table 8.1: Parameters of Problem 5.1 and Algorithm 5.1 for the SPDM.

\begin{tabular}{|c|c|c|c|}
\hline & Problem 5.1 Parameters & & \\
\hline$m_{1}$ & $4000 \mathrm{~kg}$ & $r_{s}$ & $0.0 \mathrm{~m}$ \\
\hline$m_{2}$ & $40 \mathrm{~kg}$ & $k_{1}$ & 0.0447 \\
\hline$\rho_{e}$ & {$[2.5,2.51] \mathrm{m}$} & $k_{2}$ & 0.006 \\
\hline$k_{e}$ & {$\left[10^{7}, 5 \cdot 10^{8}\right] \mathrm{N} / \mathrm{m}$} & $f_{s}^{d}$ & $-300 \mathrm{~N}$ \\
\hline$k_{s}$ & $2.1 \cdot 10^{7} \mathrm{~N} / \mathrm{m}$ & $\rho_{0}$ & $0.628 \mathrm{~m}$ \\
\hline $\bar{k}$ & {$\left[6.774 \cdot 10^{6}, 2.015 \cdot 10^{7}\right] \mathrm{N} / \mathrm{m}$} & $\mu_{1}$ & $0.3 \mathrm{~m} / \mathrm{s}$ \\
\hline$k_{p y}$ & 4 & $k_{p \phi}$ & 1 \\
\hline$k_{d u}$ & 4 & $k_{d \phi}$ & 2 \\
\hline
\end{tabular}

Algorithm 5.1 Parameters

\begin{tabular}{llllll}
\hline \hline$f_{s w}$ & $-10 \mathrm{~N}$ & $\mu_{s w}$ & $0.8 \mathrm{~mm} / \mathrm{s}$ & $\rho_{1}$ & $2.48 \mathrm{~m}$ \\
$\bar{\mu}_{s w}$ & $0.863 \mathrm{~mm} / \mathrm{s}$ & $\mu_{2}$ & $0.9 \mathrm{~mm} / \mathrm{s}$ & $\rho_{2}$ & $2.66 \mathrm{~m}$ \\
& & & & $\rho_{3}$ & $2.1 \mathrm{~m}$
\end{tabular}




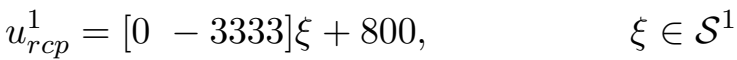

$$
\begin{aligned}
& u_{r c p}^{2}=\left[\begin{array}{ll}
0 & -3333] \xi+800, \quad \xi \in \mathcal{S}^{2}
\end{array}\right. \\
& u_{r c p}^{3}=[-9200-3333] \xi+19200, \quad \xi \in \mathcal{S}^{3} \\
& u_{r c p}^{4}=[-3600-5200] \xi+8000, \quad \xi \in \mathcal{S}^{4} \\
& u_{r c p}^{5}=[-1154-5200] \xi+2864, \quad \xi \in \mathcal{S}^{5} \\
& u_{r c p}^{6}=[-1154-5200] \xi+2864, \quad \xi \in \mathcal{S}^{6} \\
& u_{r c p}^{7}=[-1154-3996] \xi+2864, \quad \xi \in \mathcal{S}^{7}
\end{aligned}
$$

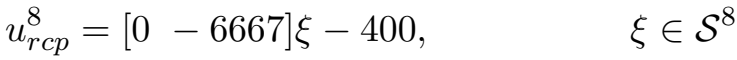

$$
\begin{aligned}
& u_{r c p}^{9}=\left[\begin{array}{ll}
0 & -6667
\end{array}\right] \xi-400 \quad \xi \in \mathcal{S}^{9} \\
& u_{r c p}^{10}=[-83.3-22222] \xi+221.7 \quad \xi \in \mathcal{S}^{10}
\end{aligned}
$$

The simplified closed-loop vector field under the reach controllers in $\mathcal{P}$ is given in Figure 8.1 (note that $\mathcal{P}_{3}$ is too small to see). Clearly, the closed-loop trajectories are driven into $\mathcal{P}^{2}$, which will then be guided into $\mathcal{P}^{3}$ where contact is made with the environment (as marked by the vertical dashed line). Finally, the overall hybrid controller is constructed as in (6.1) using the reach controllers above and the force controller (7.75).

\subsection{Simulations}

In this section we simulate the SPDM system and evaluate the proposed design. First, a set of base tests will be performed to demonstrate the desired behaviour under nominal conditions. Then, we explore how parameter changes amongst different dimensions of the system affect the closed-loop behaviour.

\section{Base Tests}

We begin with a set of base tests where the environment position is set to $\hat{\rho}_{e}=3.008 \mathrm{~m}\left(\rho_{e}=\right.$ $2.505 \mathrm{~m}$ ), and three environment stiffnesses are selected over its nominal range: $k_{e}=10^{7}, 2.1$. $10^{8}, 5 \cdot 10^{8} \mathrm{~N} / \mathrm{m}$. For these tests the manipulator was initialized at rest with joint angles 


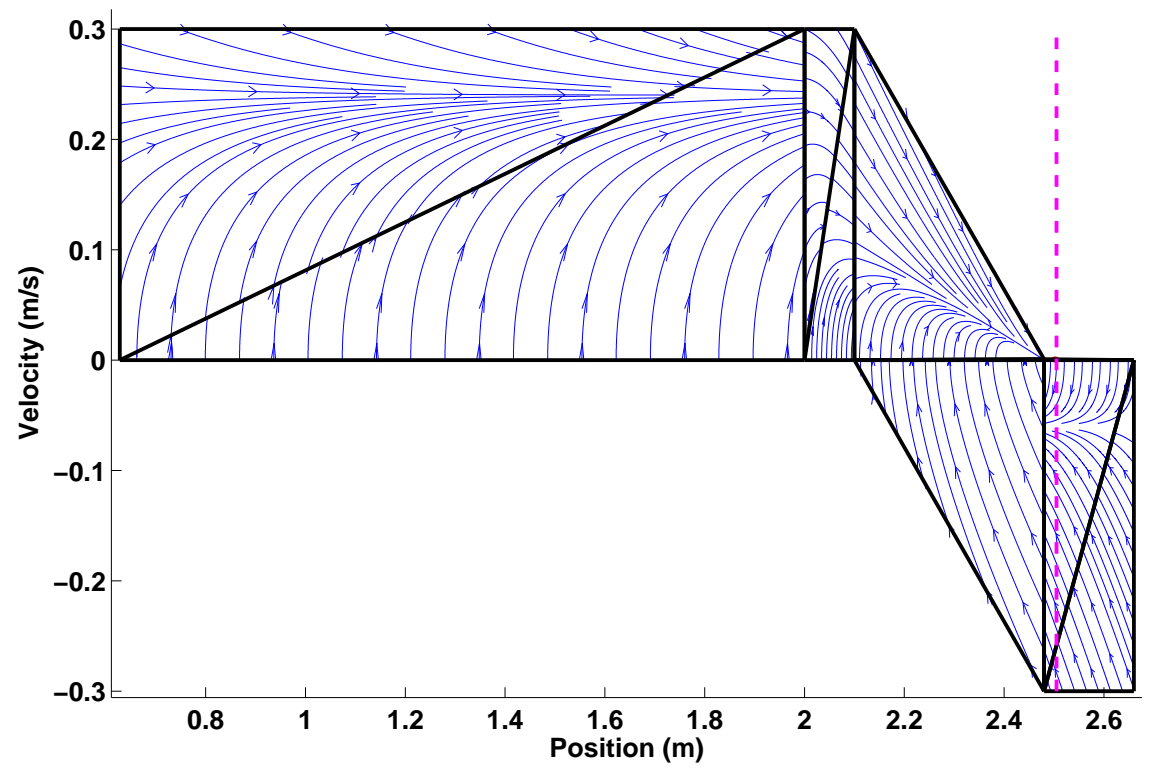

Figure 8.1: Closed-loop vector field of $\mathcal{P}$ under $u_{r c p}$.

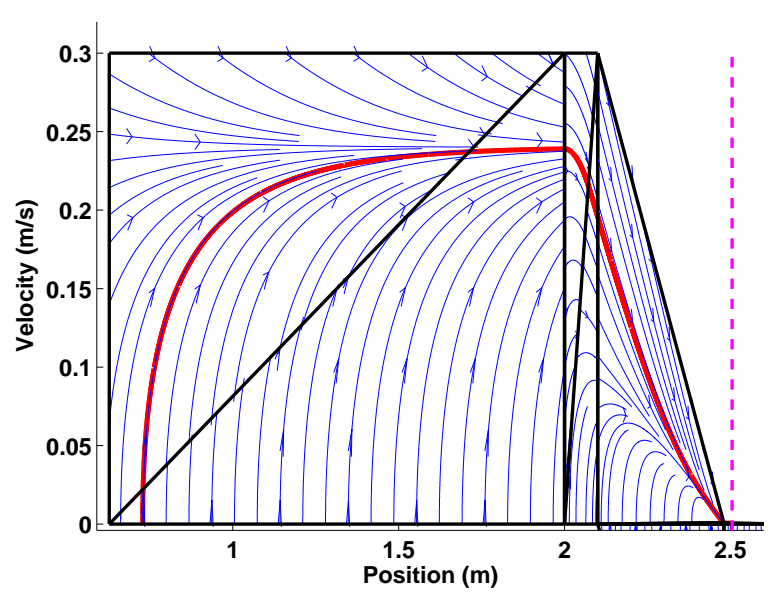

(a)

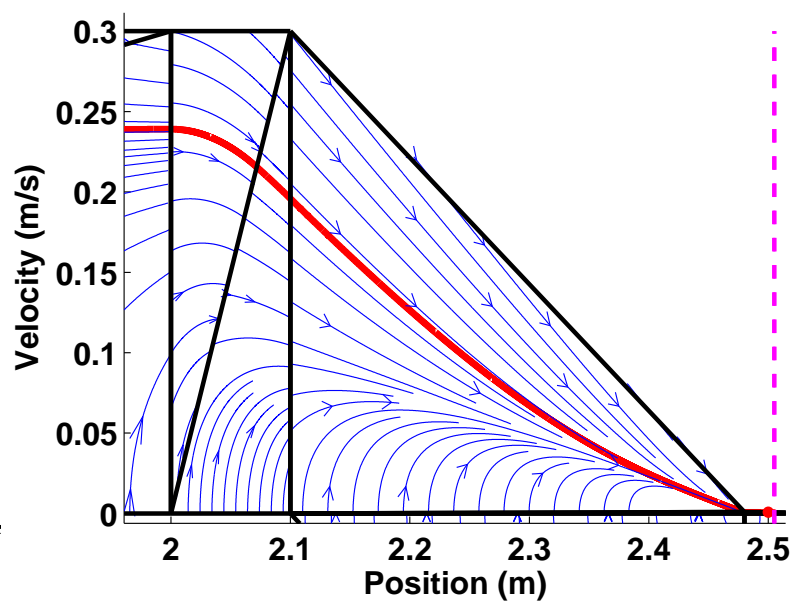

(b)

Figure 8.2: (a),(b) Trajectory of $\xi$ of the first mass in $\mathcal{P}^{1}$ and $\mathcal{P}^{2}$.

$\theta(0)=\left(155^{\circ},-95^{\circ},-25^{\circ}\right)$, which correspond to initial conditions $\left(x_{1}, y_{1}\right)=(0.728,1.44) \mathrm{m}$, $\left(x_{2}, y_{2}\right)=(1.14,1.73) \mathrm{m}$, and $\phi(0)=35^{\circ}$. Moreover, we select $y_{1}^{d}=1 \mathrm{~m}$. The reach control phase of the maneuver is depicted in Figures 8.2-8.3, wherein the red curve represents the trajectory of $\xi$ through $\mathcal{P}$. Clearly the reach controllers induce the expected behaviour, ensuring that the safety and liveness constraints of $\mathcal{P}$ are met. In Figure 8.2 the end effector travels 


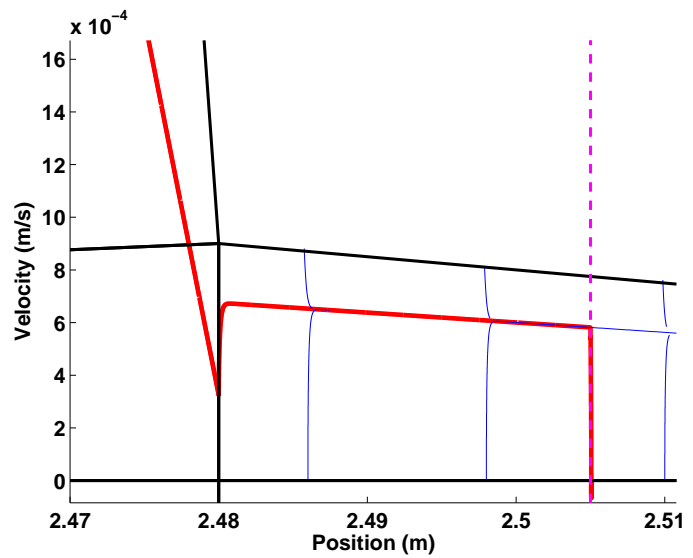

Figure 8.3: Trajectory of $\xi$ of the first mass in $\mathcal{P}^{3}$.

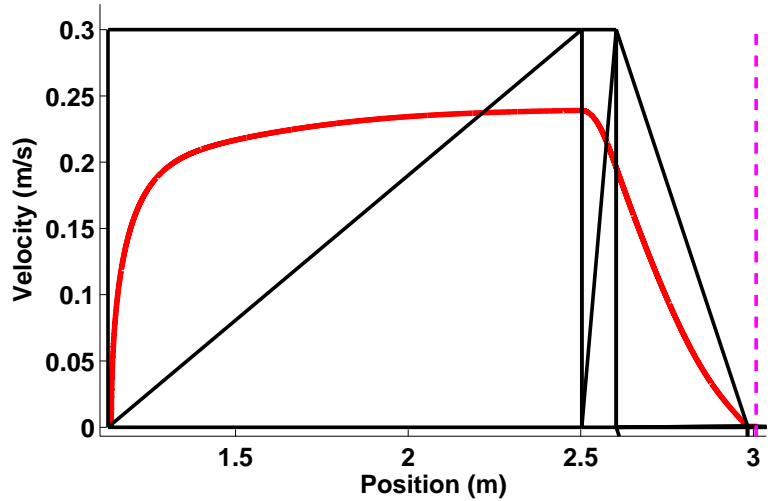

(a)

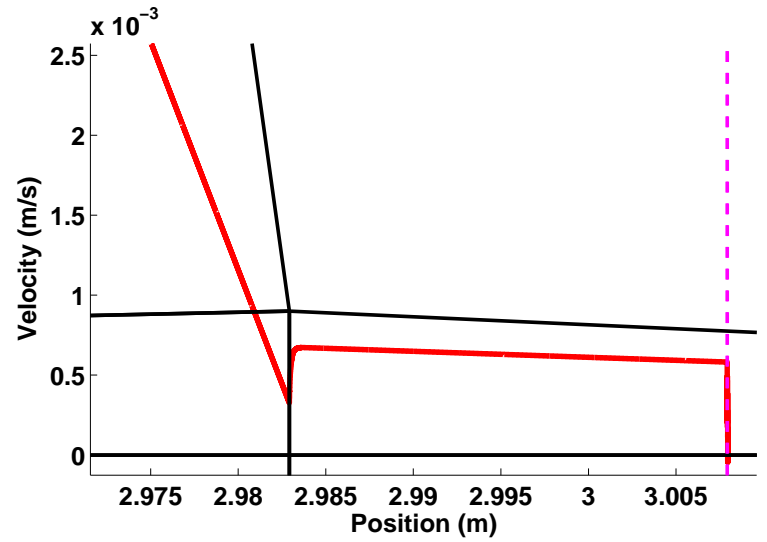

(b)

Figure 8.4: (a) Trajectory of $\left(x_{2}, \dot{x}_{2}\right)$ of the second mass in $\mathcal{P}^{1}$ and $\mathcal{P}^{2}$. (b) Trajectory of $\left(x_{2}, \dot{x}_{2}\right)$ of the second mass in $\mathcal{P}^{3}$.

at high velocity through $\mathcal{P}^{1}$, and begins to decelerate through $\mathcal{P}^{2}$ as it nears the environment. Referring to Figure 8.3, which depicts $\mathcal{P}^{3}$, the reach controller $u_{r c p}^{10}$ slowly guides the end effector of the manipulator towards the environment with decreasing velocity until impact is made with velocity $0.58 \mathrm{~mm} / \mathrm{s}$ (which is well below $\mu_{s w}=0.8 \mathrm{~mm} / \mathrm{s}$ ). Similar behaviour for the second mass states $\left(x_{2}, \dot{x}_{2}\right)$ is observed in Figure 8.4. In contrast to the previous manipulator system, the trajectory of $\left(x_{2}, \dot{x}_{2}\right)$ is not oscillatory because of the light structural damping that is present in the system model.

Figures 8.5-8.7 depict the transition and constrained motion phases of the maneuver for $\xi$, $\left(x_{2}, \dot{x}_{2}\right)$, and sensed force $f_{s}$ for the three tests (ordered from highest to lowest values of $k_{e}$ ). 


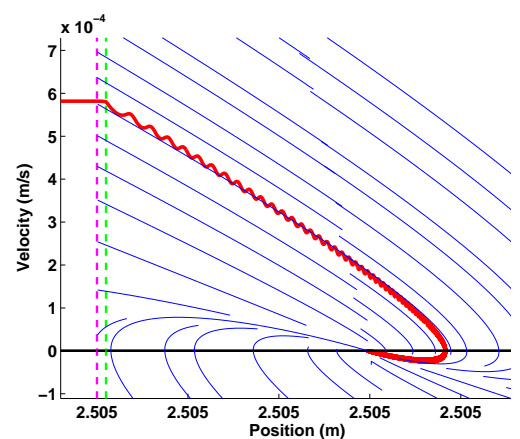

(a)

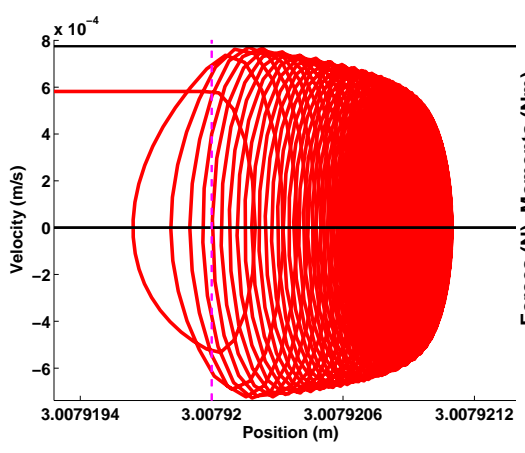

(b)

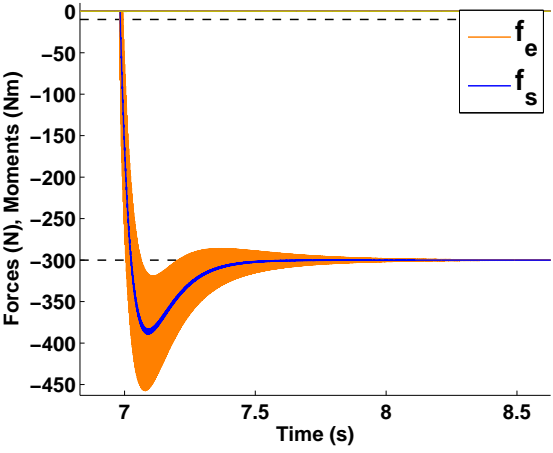

(c)

Figure 8.5: Highest environment stiffness $k_{e}=5 \cdot 10^{8} \mathrm{~N} / \mathrm{m}$ : (a) Constrained trajectory of $\xi$. (b) Constrained trajectory of $\left(x_{2}, \dot{x}_{2}\right)$. (c) Sensed force $f_{s}$ and environmental force $f_{e}$.

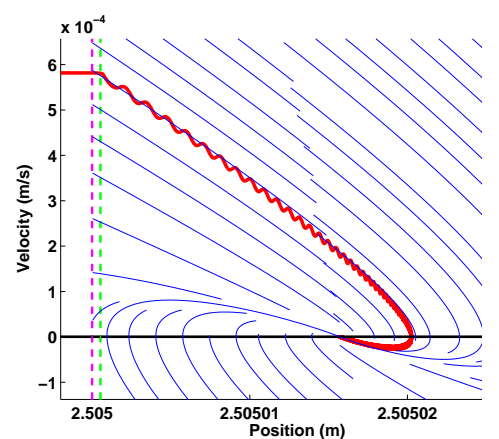

(a)

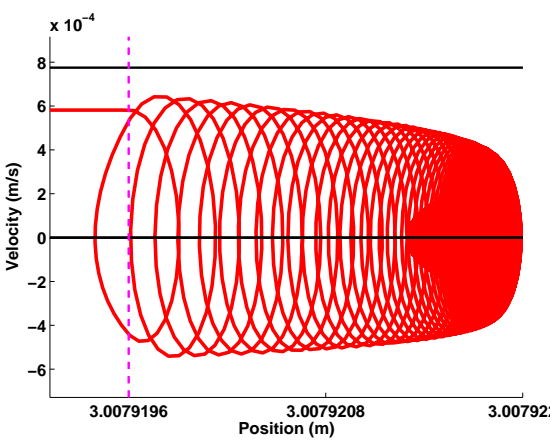

(b)

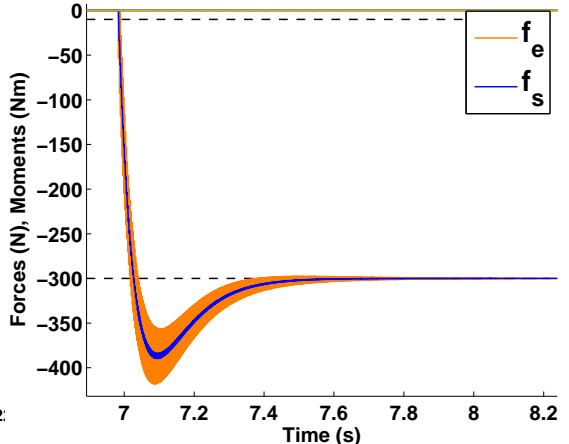

(c)

Figure 8.6: Mid-range environment stiffness $k_{e}=2.1 \cdot 10^{8} \mathrm{~N} / \mathrm{m}$ : (a) Constrained trajectory of $\xi$. (b) Constrained trajectory of $\left(x_{2}, \dot{x}_{2}\right)$. (c) System forces $f_{s}$ and $f_{e}$.

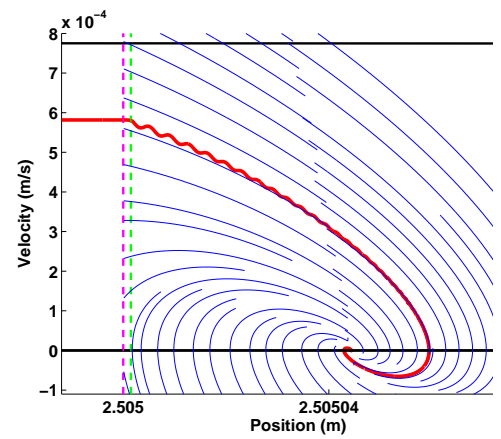

(a)

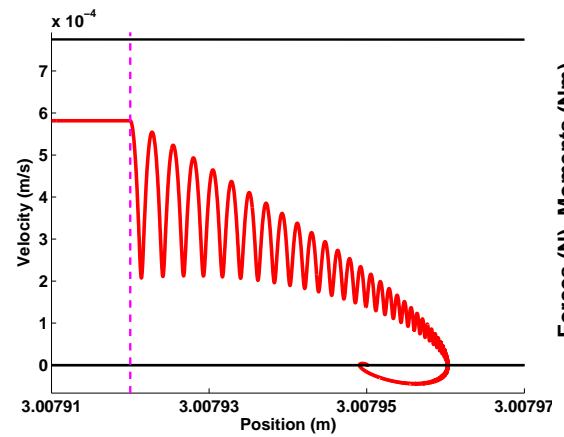

(b)

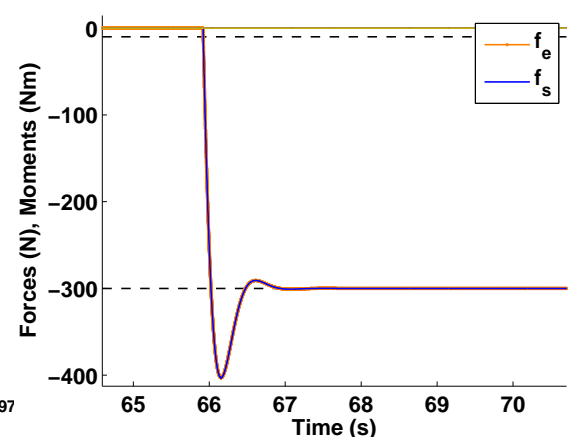

(c)

Figure 8.7: Lowest environment stiffness $k_{e}=10^{7} \mathrm{~N} / \mathrm{m}$ : (a) Constrained trajectory of $\xi$. (b) Constrained trajectory of $\left(x_{2}, \dot{x}_{2}\right)$. (c) System forces $f_{s}$ and $f_{e}$. 
Recall that $f_{s}$ is the sensed force in the $x$-direction measured with respect to the base frame. Annotations in these graphs are similar to those of Chapter 6, except here the orange waveform on the right is the environment force $f_{e}$. Referring to the graphs on the left, plots (a), $u_{r c p}^{10}$ guides $\xi$ to the switching boundary. A switch to force control then occurs and the corresponding states asymptotically approach a state associated with $f_{s}^{d}$, as observed in plots (a) and (b) (the convergence is difficult to see in Figures 8.5-8.6 because of the oscillations, and the former takes longer). The evolution of $\xi$ closely matches the overlaid simplified dynamics, but differs slightly due to the light structural damping that was not considered in the controller design. In the graphs on the right, plots (c), the sensed force is regulated to $f_{s}^{d}=-300 \mathrm{~N}$ without crossing back over $f_{s w}=-10 \mathrm{~N}$, achieving a bumpless transfer. Therefore, the temporal sequence of Problem 5.1 has been achieved using the proposed state space $\mathcal{P}$, reach controllers, and hybrid switching system.

Other interesting behaviour may be deduced from these graphs as well, namely the behaviour of $\left(x_{2}, \dot{x}_{2}\right)$ and the forces when the ratio $k_{e} / k_{s}$ is large. When $k_{e}>k_{s}$ the SPDM is compliant due to the modelling of the translational displacement $\delta_{x}$ between the first and second masses. This modelling function allows the second mass to visibly oscillate. The obvious reason for the oscillation is that the second mass is bouncing off the surface of the environment (about four times when $k_{e}=5 \cdot 10^{8} \mathrm{~N} / \mathrm{m}$ and once when $k_{e}=2.1 \cdot 10^{8} \mathrm{~N} / \mathrm{m}$ ), and so the environment force is also quite oscillatory. Conversely, when $k_{e}<k_{s}$ the environment is compliant, the motion of the first and second masses are similar, and therefore the environment force is not oscillatory. The oscillatory behaviour is a characteristic limitation of the proposed design, in which the dynamics of the second mass were generally neglected, and the compliance of the sensor was considered in the motion of the masses. Recall that these assumptions allowed the sensed force $f_{s}$ to be expressed in terms of $\xi$ and an effective spring constant $\bar{k}$, which in turn produced the force model (7.76e). Consequently, the relationship between $\xi$ and $f_{s}$ allowed for the derivation of conditions that prevent a bounce in the control (see the Lyapunov analysis of Lemmas 5.2 and 5.3), which is the desirable behaviour observed in these waveforms.

Lastly, we are interested in the measured force in the remaining degrees of freedom in the plane of motion, i.e. the measured force in the $y$-direction, $F_{y}$, and the moment about the $z$ axis, $M_{z}$. Sample waveforms of these signals are depicted in Figure 8.8. The initial spikes in the 


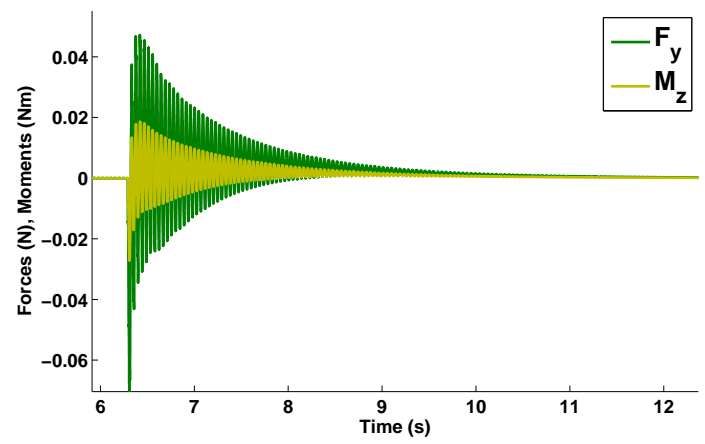

Figure 8.8: FMS sensed force $F_{y}$ and moment $M_{z}$ during contact.

waveforms are due to the initial impact of the end effector with the environment, which induce minor misalignments in $y_{1}$ and $\phi$. However, the lack of force control in these directions, and the inherent stiffness and damping defined for the FMS joints, result in these forces autonomously decaying to zero.

Moving on we consider additional relevant waveforms for the case where $k_{e}=10^{7} \mathrm{~N} / \mathrm{m}$, which are representative of the simulations that follow. In particular, Figure 8.9 depicts which sub-controller of $u\left(\xi, f_{s}\right)$ is active as a function of time, and the magnitudes of the joint torques $\tau_{1}, \tau_{2}, \tau_{3}$, which closely resemble those given in Chapter 6 . We note that for the SPDM, the light structural damping essentially eliminates oscillations of the sensed force, and so no spurious switching is observed between reach controllers $u_{r c p}^{5}$ and $u_{r c p}^{6}$ in the graph on the left. Also, the motion is completed in a timely manner as the states reach $\mathcal{P}^{3}$ after $26 \mathrm{~s}$, and a switch to $u_{f}$ occurs at 66s. Referring to plot (b), similar comments to those in Chapter 6 can be made in regards to the behaviour of the torque waveforms. In particular, the initial spikes lasting from about zero to four seconds are a result of the PD controllers regulating $y_{1}$, and $\phi$ to their reference values, while the reach controllers accelerate the system forward in the $x$-direction. Then, the spike at around 8s corresponds to the system entering $\mathcal{P}^{2}$ and quickly decelerating the system according to the diagonal safety constraint of Figure 5.2. Again we consider the notion that the size of $\mathcal{P}^{2}$ contributes to the spike in torque, as the actuators must decelerate the system from high to low velocity over a short distance. Recall that the size of $\mathcal{P}^{2}$ depends in part on the selection of parameter $\rho_{3}$. Finally, the last feature of the torque waveforms is the spike at around $66 \mathrm{~s}$, which is when the force controller is activated. 


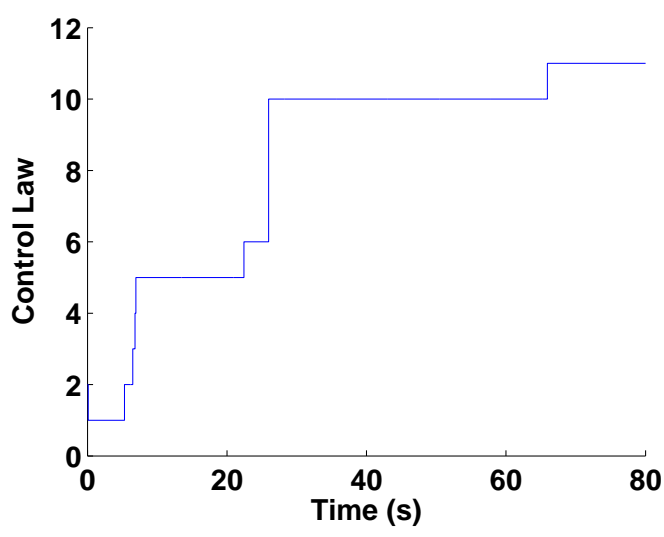

(a)

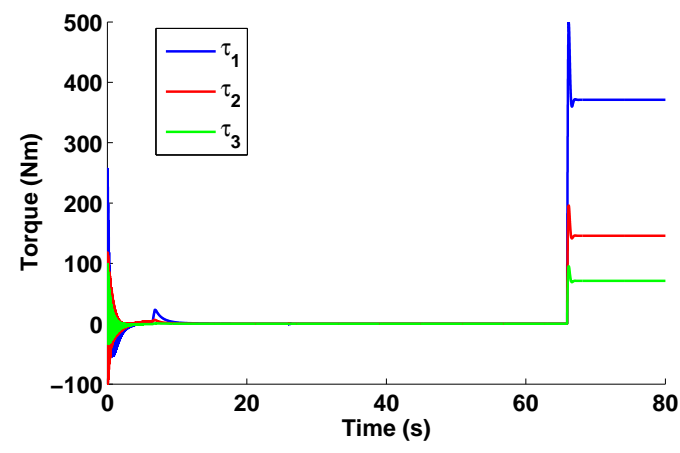

(b)

Figure 8.9: (a) Index of active sub-controller of $u\left(\xi, f_{s}\right)$. (b) Manipulator joint torques $\tau$.

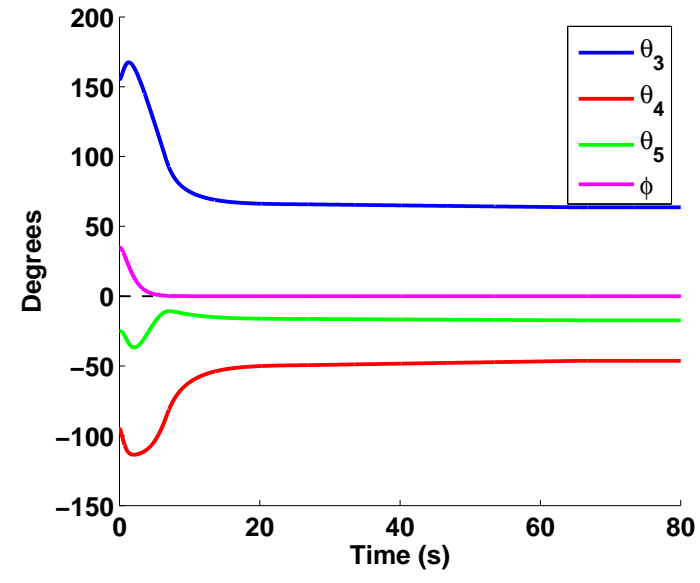

(a)

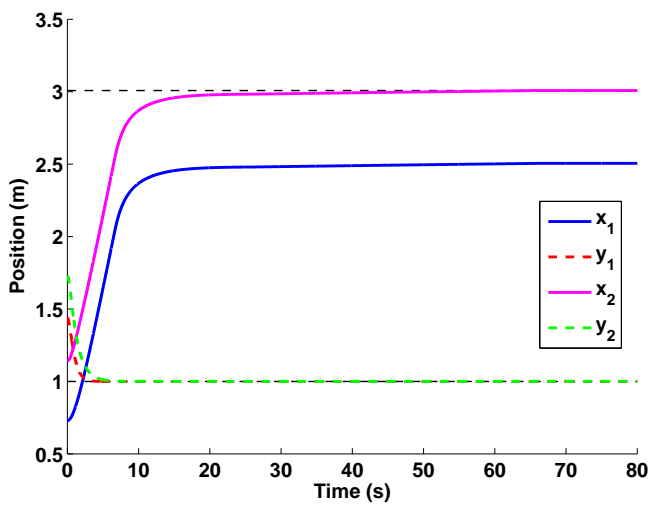

(b)

Figure 8.10: (a) Manipulator joint angles $\theta$ and orientation angle $\phi$. (b) Cartesian coordinates of the first mass $x_{1}, y_{1}$ and second mass $x_{2}, y_{2}$.

Finally, Figure 8.10 depicts the regulation of the task space variables $\phi$ and $y_{1}$. On the left is the evolution of the joint variables $\theta_{i}$, along with the central magenta line representing $\phi$. Clearly, regulation of $\phi$ to $\phi^{d}=0^{\circ}$ occurs, which ensures a horizontal approach to the environment. On the right is the time evolution of $x_{2}, y_{2}$ of the second mass and $x_{1}, y_{1}$ of the first mass. We have that the $y_{i}$ readily approach $y_{1}^{d}=1 \mathrm{~m}$, while the $x_{i}$ slowly approach their corresponding environment positions $\hat{\rho}_{e}$ and $\rho_{e}$.

Next, we perform a functional simulation wherein the manipulator is made to lose contact with the environment (either on command or due to a disturbance). The purpose of the test 


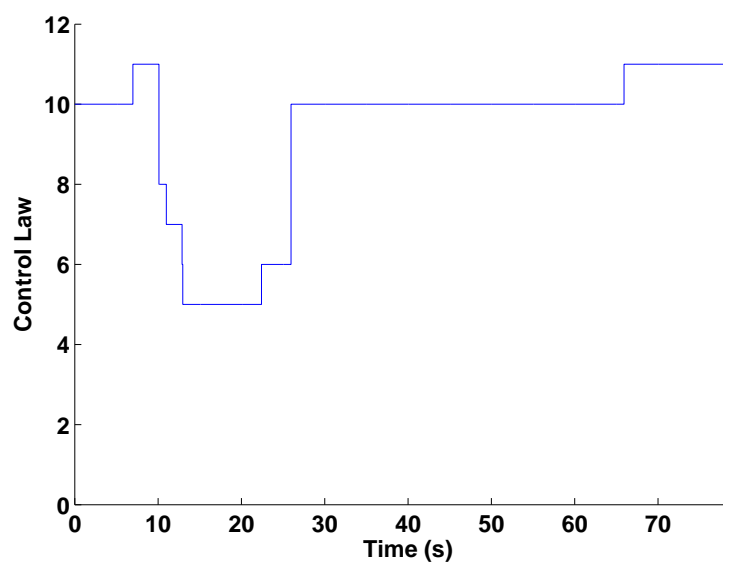

(a)

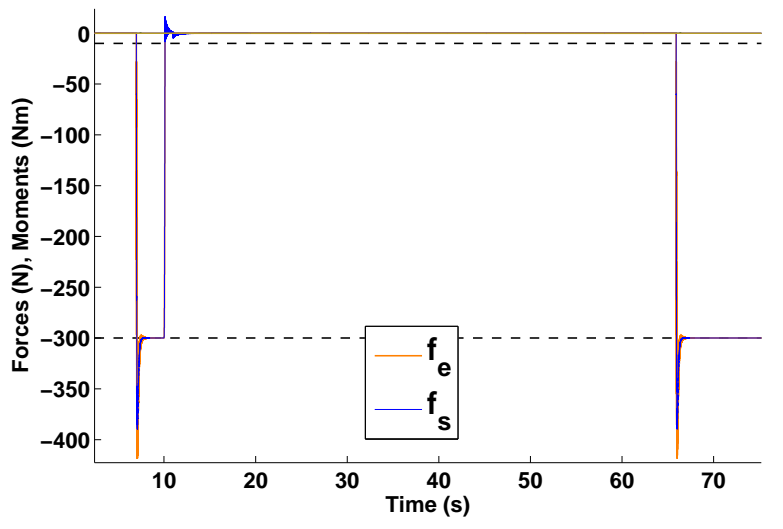

(b)

Figure 8.11: Return Approach: (a) State of control law $u\left(\xi, f_{s}\right)$. (b) System forces $f_{s}$ and $f_{e}$.

is to demonstrate the capability of the hybrid control system to re-establish contact with the environment. Figures 8.11 and 8.12 depict the state of the hybrid control, the forces of interest, and the trajectory of $\xi$ during the sequence. First, the manipulator was initialized close to the environment and driven to establish stable contact at the desired force, i.e. see the first 10s in Figure 8.11 when the hybrid control state is 10 and 11. At approximately 10s, the manipulator is driven backwards to imitate the loss of contact, for example plot (b) shows the sensed force decreasing in magnitude. Eventually, the switching force threshold is tripped in the opposite direction, and since the manipulator is moving backwards $\xi_{2}$ is negative, so the states $\xi$ are located within $\mathcal{S}^{8}$ of $\mathcal{P}^{4}$. Therefore, reach controller $u_{r c p}^{8}$ is enabled. Then the trajectory of $\xi$ is driven backwards through $\mathcal{P}^{4}$ and $\mathcal{P}^{5}$ as the control repositions the manipulator to repeat the maneuver, as seen in Figure 8.12. Afterwards, the same sequence of events as the previous base tests occurs: the hybrid controller steps through the various reach controllers and switches to force controller $u_{f}$ at $66 \mathrm{~s}$; similarly the sensed force is regulated again to $f_{s}^{d}=-300 \mathrm{~N}$.

Overall, this set of experiments is considered successful and indicative of the desired behaviour that solves Problem 5.1. As indicated however, excessive oscillations in the environment force and repeated bouncing of the second mass occurs when the ratio $k_{e} / k_{s}$ is large, seen here when $k_{e}=5 \cdot 10^{8} \mathrm{~N} / \mathrm{m}$. Thus, the environment stiffness $k_{e}=2.1 \cdot 10^{8} \mathrm{~N} / \mathrm{m}$ will be used for all subsequent tests unless otherwise stated, because it represents a scenario where the environment is reasonably stiffer than the sensor, while yielding an acceptable level of oscillations 


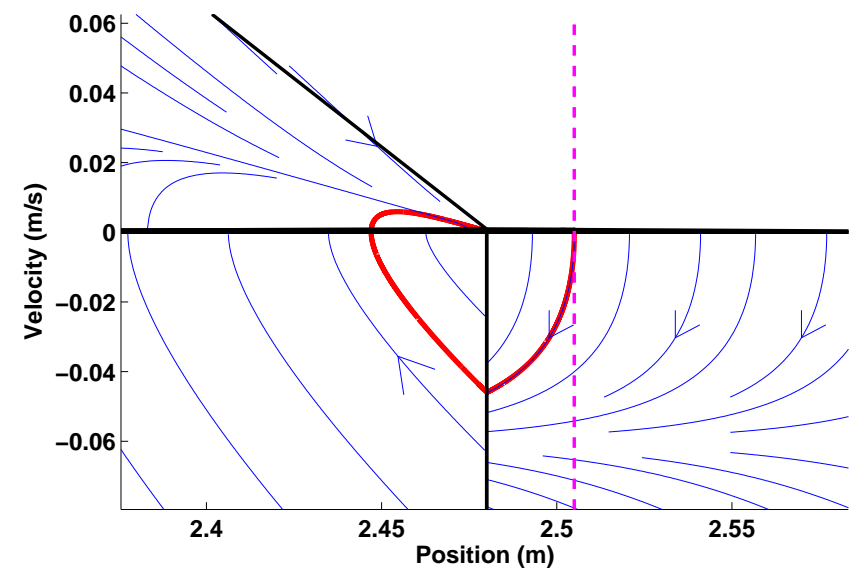

Figure 8.12: Return Approach: Closed-loop vector trajectory of $\xi$.

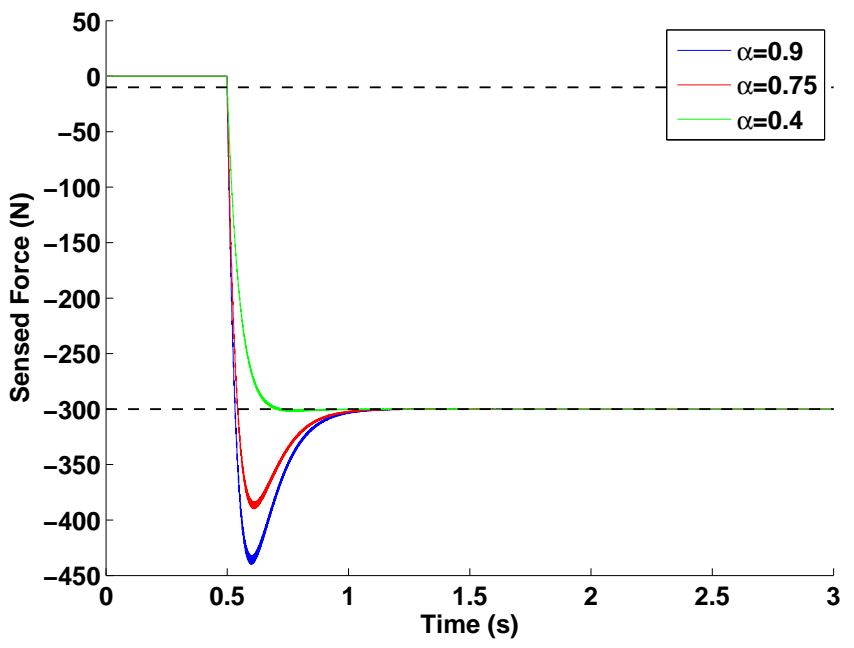

Figure 8.13: Sensed force $f_{s}$ for varying reach control design parameter $\alpha$.

and bouncing. As in Chapter 6 we will explore the effects of a change in control parameters, manipulator features, and the environment.

\section{Controller Modifications}

As in Chapter 6 we observe how changes in the proposed controllers, specifically the design of reach controller $u_{r c p}^{10}$ and the mass ratio $m_{1} / m_{2}$ of the feedback linearisation, influence the behaviour of the system. Starting with $u_{r c p}^{10}$, recall from the simulations of Chapter 6 that $u_{r c p}^{10}$ depends in part on the parameter $\alpha$ where $\alpha=\frac{u_{7}^{3}}{u_{7}^{3}-u_{6}^{3}}$. As $\alpha$ is decreased trajectories in $\mathcal{P}^{3}$ travel slower and the impact velocity is reduced, which had the consequence of reducing the peak contact force during force control. The response $f_{s}$ for $\alpha=0.4,0.75,0.9$ is depicted in 


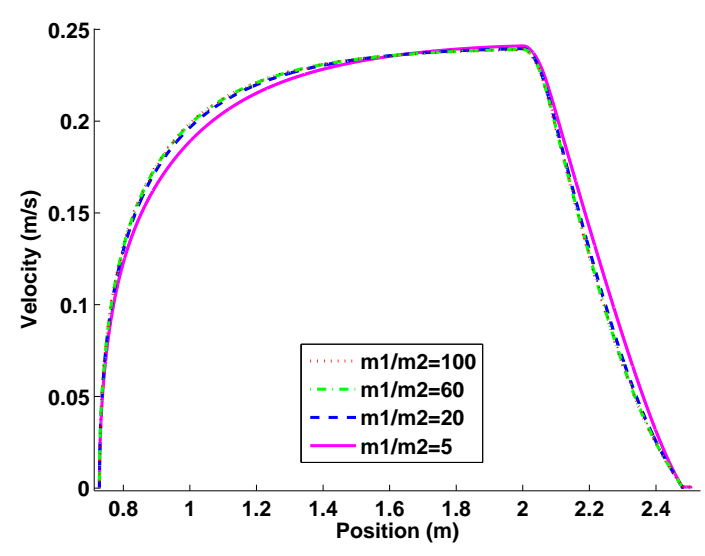

(a)

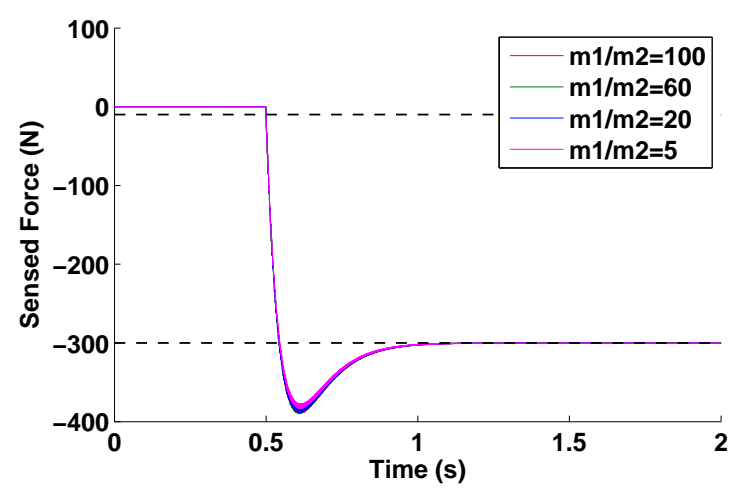

(b)

Figure 8.14: (a) Trajectories $\xi$ of the first mass in $\mathcal{P}^{1}$ and $\mathcal{P}^{2}$ for different mass ratios. (b) The sensed force $f_{s}$ for different mass ratios.

Figure 8.13. The same trend between $\alpha$ and the peak contact force is clearly visible. From smallest to largest the impact velocities for these tests were $0.31 \mathrm{~mm} / \mathrm{s}, 0.58 \mathrm{~mm} / \mathrm{s}$, and $0.7 \mathrm{~mm} / \mathrm{s}$, which correspond respectively to reach controllers

$u_{r c p}^{10}=\left[\begin{array}{ll}-83.3-41667\end{array}\right] \xi+221.7, \quad u_{r c p}^{10}=\left[\begin{array}{ll}-83.3 & -22222\end{array}\right] \xi+221.7, \quad u_{r c p}^{10}=\left[\begin{array}{lll}-83.3 & -18519\end{array}\right] \xi+221.7$.

Therefore under the proposed methodology the design of $u_{r c p}^{10}$ can limit the peak contact force.

Next, the mass ratio was varied by changing the parameter $m_{1}$ to satisfy $m_{1} / m_{2}=60,20,5$. We remark that the resulting reach and force controllers saw their parameters scaled by $60 \%$, $20 \%$, and $5 \%$ respectively from the nominal controllers (except for $u_{r c p}^{10}$ ). Figure 8.14 (a) depicts four overlaid trajectories of $\xi$ under reach control for the different mass ratios (the base case is included) as they travel through $\mathcal{P}^{1}$ and $\mathcal{P}^{2}$ (whose edges are not explicitly shown). Clearly the trajectory pertaining to $m_{1} / m_{2}=5$ stands out, while the other three cases do not vary significantly. This implies that a designer may select a relatively small mass ratio before the model used to design the reach controllers, (7.76a), is invalidated. Similarly for constrained motion, Figure 8.14 (b) depicts the sensed force response $f_{s}$ overlaid for all four cases. Again we observe little difference between cases as a bumpless transfer to force control is achieved throughout. Recall though that some of the dynamics pertaining to inertial forces and configuration changes are generally removed in the SPDM simulator, [5][38], and that their 


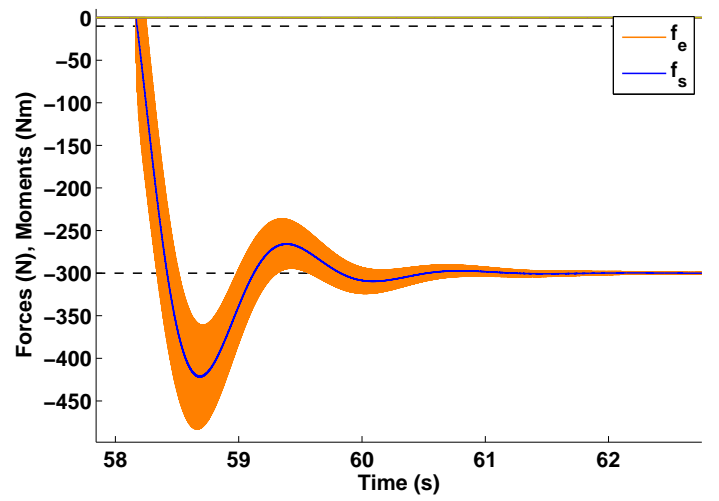

(a)

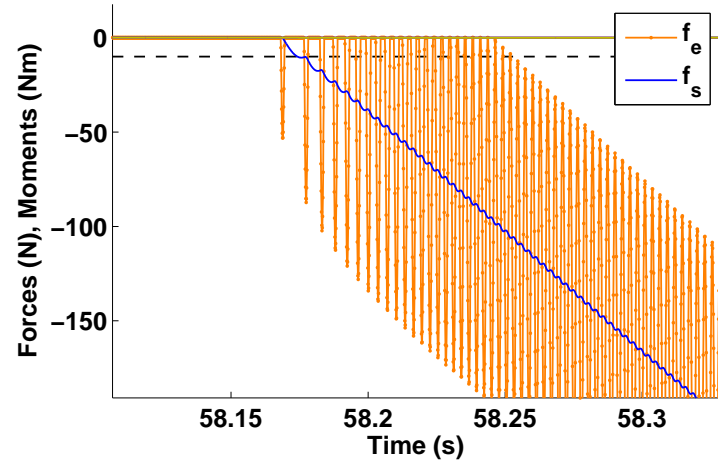

(b)

Figure 8.15: Below nominal sensor stiffness $k_{s}=2.1 \cdot 10^{6} \mathrm{~N} / \mathrm{m}$ : (a) System forces $f_{s}$ and $f_{e}$. (b) System forces $f_{s}$ and $f_{e}$ close to the switching boundary.

inclusion may require the mass ratio to be larger than 5 for the design assumptions to hold.

While this section only discussed the modification of reach controller $u_{r c p}^{10}$, in which contact first occurs, the remaining controllers can obviously be changed to generate different free motion trajectories while still adhering to the logic control specifications. With regards to constrained motion, a change in the architecture of the force controller itself is a topic deferred to the last chapter of this thesis where future work is discussed.

\section{SPDM Variations}

Now we turn our attention to the manipulator itself, and how variations in the sensor stiffness $K_{\delta}$ and the structural damping matrix $D_{\delta}$ influence the closed-loop behaviour. First we assume that the sensor stiffness has degraded from its nominal value by a factor of 10 such that $k_{s}=$ $2.1 \cdot 10^{6} \mathrm{~N} / \mathrm{m}$ and $k_{e} / k_{s}=100$. Figure 8.15 depicts the responses of $f_{s}$ and $f_{e}$ obtained from the simulation, with particular focus around the switching boundary in plot (b). Clearly, a bumpless transfer is still achieved, but the extreme difference in stiffnesses admits excessive bouncing of the second mass. Moreover, the large oscillations of the second mass induce peak environment forces of almost $500 \mathrm{~N}$, which is undesirable. Therefore, while successful, this test further demonstrates the limitations of the design when $k_{e} / k_{s}$ is large.

Next we address structural damping of the FMS joints. In all of the simulations performed thus far a damping factor of $z=0.005$ has been used, representing extremely light damping. 


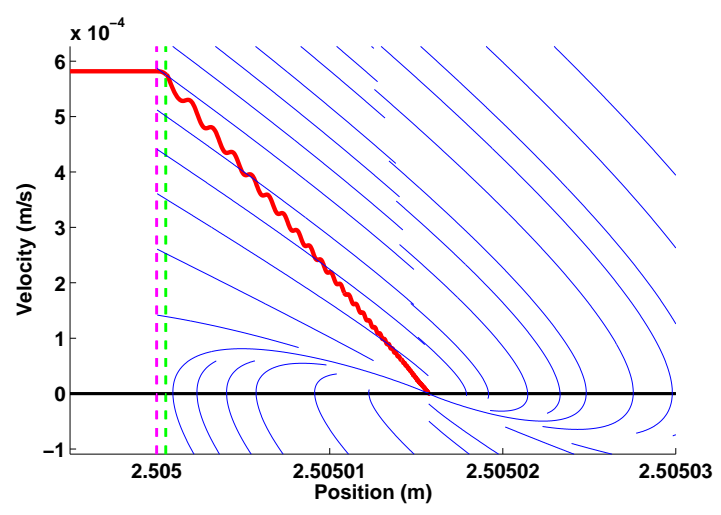

(a)

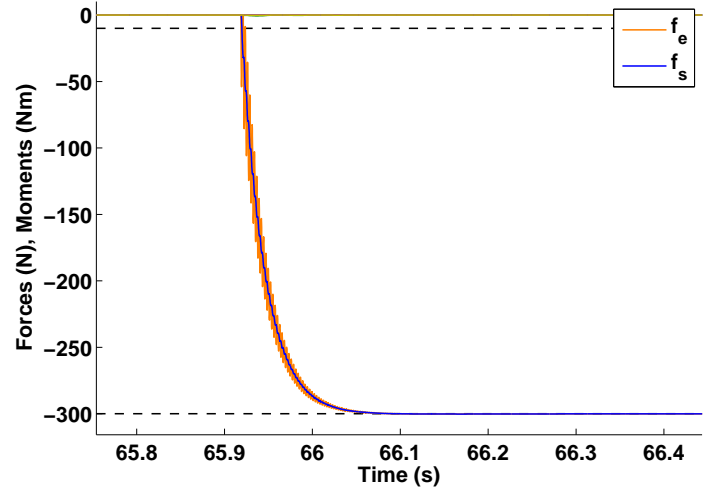

(b)

Figure 8.16: (a) Constrained motion of $\xi$ for greater structural damping, $z=0.05$. (b) System forces $f_{s}$ and $f_{e}$ for greater structural damping, $z=0.05$.

Instead here we simulate the system under a more realistic scenario by setting $z=0.05$, the nominal value given in the SPDM simulator, while retaining the original reach and force controllers. Figure 8.16 depicts the case where $z=0.05$, which can be can be directly compared to Figure 8.6 in which $z=0.005$. Referring to plot (a) in both figures, the constrained evolutions of $\xi$ clearly approach the equilibrium $\xi_{f}^{d}=\left(\rho_{f}^{d}, 0\right)$ associated with $f_{s}^{d}=-300 \mathrm{~N}$; however, for greater damping the trajectory does not conform to the overlaid force dynamics. This follows from the fact that inherent damping in the definition of the sensed force was not originally considered. In regards to the force waveforms (plot (b) here and plot (c) from the base test), we observe that $f_{e}$ is less oscillatory and the peak contact forces are greatly reduced as a result of increased damping; this is as expected. The main observation to be gleaned from this test is that if the structural damping term of the FMS joints is greater than that assumed during the design phase, then a successful bumpless transfer to force control is still possible. However, the more appropriate course of action, and a possible avenue for future work, is to develop a methodology akin to Chapter 5 in which the sensed force is characterized by stiffness and damping terms. 


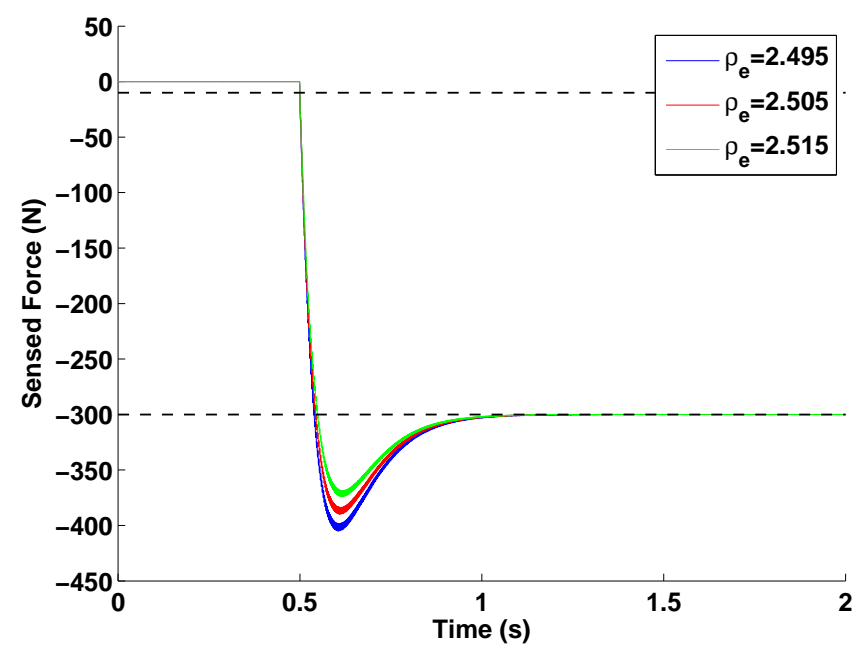

Figure 8.17: Sensed force $f_{s}$ for varying environment position $\rho_{e}$.

\section{Environment Variations}

Lastly, the SPDM is simulated under conditions where the environment position $\hat{\rho}_{e}$ and stiffness $k_{e}$ are allowed to vary outside of their respective nominal ranges. We first examine the effects of the variation in position. Two simulations were performed where $\hat{\rho}_{e}=\hat{\rho}_{\text {emin }}-0.005$ and $\hat{\rho}_{e}=\hat{\rho}_{\text {emax }}+0.005$, with the response of the sensed force $f_{s}$ given in Figure 8.17 (the base case test is also included). Note that from largest to smallest the impact velocities for these tests were: $0.62 \mathrm{~mm} / \mathrm{s}, 0.58 \mathrm{~mm} / \mathrm{s}$, and $0.54 \mathrm{~mm} / \mathrm{s}$. In all cases it is clear that the reach controller in $\mathcal{P}^{3}$ successfully guides trajectories to the switching line, at which point a bumpless transfer to force control occurs, and the sensed force is regulated. Again as in Chapter 6, we see that the peak contact force in Figure 8.17 decreases as $\hat{\rho}_{e}$ (equivalently $\rho_{e}$ ) increases, due to the variation in the impact velocity as the environment position changes. The clear culprit of this phenomenon is the architecture of $\mathcal{P}^{3}$ itself, which is triangular in order to restrict the maximum extension of the manipulator to no further than $\xi_{1}=\rho_{2}$. If desired, one could alter this arrangement by redefining and adding to the logic control specifications to render $\mathcal{P}^{3}$ a rectangular region (a polytope). Then a triangular region (a simplex) would need to be added after $\mathcal{P}^{3}$ to constrain the maximum position. In this case the manipulator could be made to travel at constant velocity in the polytope, which would eliminate the dependence between environment position and the peak force. 


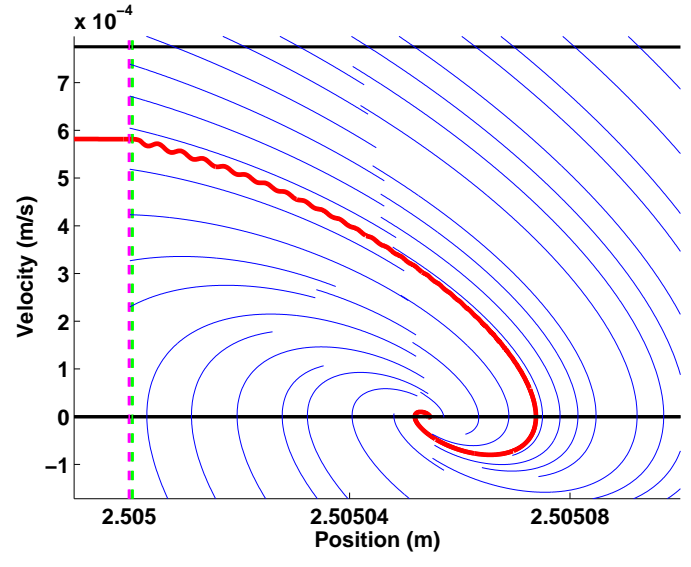

(a)

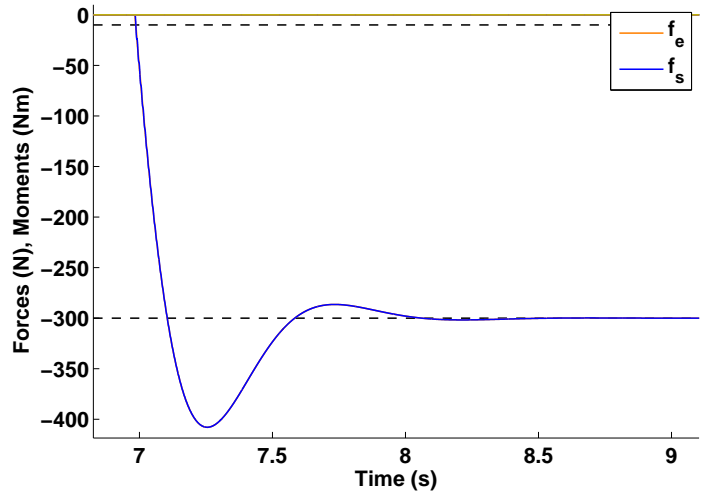

(b)

Figure 8.18: Below nominal environment stiffness $k_{e}=7.5 \cdot 10^{6} \mathrm{~N} / \mathrm{m}$ : (a) Constrained trajectory $\xi$ of the first mass. (b) System forces $f_{s}$ and $f_{e}$.

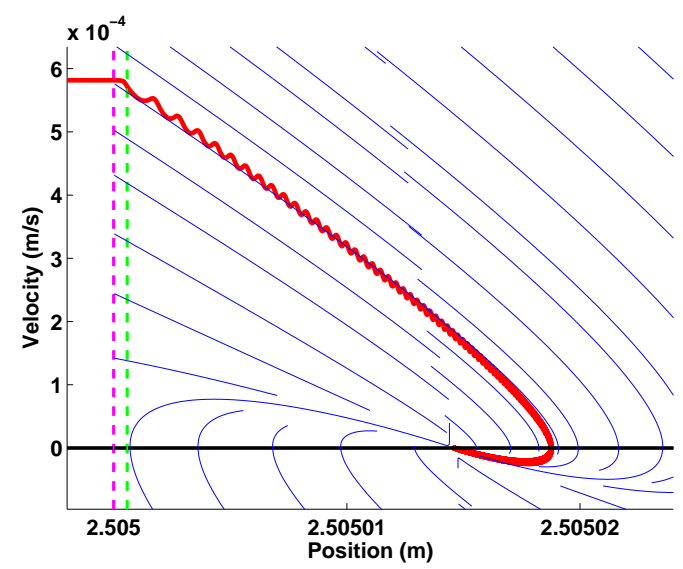

(a)

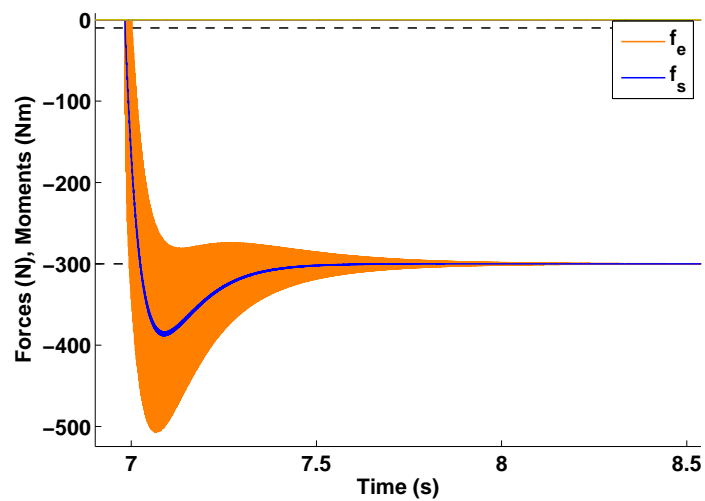

(b)

Figure 8.19: Above nominal environment stiffness $k_{e}=10^{9} \mathrm{~N} / \mathrm{m}$ : (a) Constrained trajectory $\xi$ of the second mass. (b) System forces $f_{s}$ and $f_{e}$.

Next we examine the scenario where the environment stiffness is not as expected, which causes the force dynamics to vary. Figure 8.18 depicts the transition and constrained motion phases of $\xi$ and $f_{s}$ for a softer environment, $k_{e}=7.5 \cdot 10^{6} \mathrm{~N} / \mathrm{m}$. As explained in Chapter 6 , since control $u_{7}^{3}$ was chosen to allow for variation in the term $\frac{\left|f_{s w}\right|}{\bar{k}_{\text {min }}}$, then the reach controller $u_{r c p}^{10}$ is able to guide the manipulator to the switching line. For this test the impact velocity is $0.59 \mathrm{~mm} / \mathrm{s}<\bar{\mu}_{s w}$, and so a bumpless transfer is achieved.

Alternatively, Figures 8.19 and 8.20 depict the case where the environment is stiffer than 


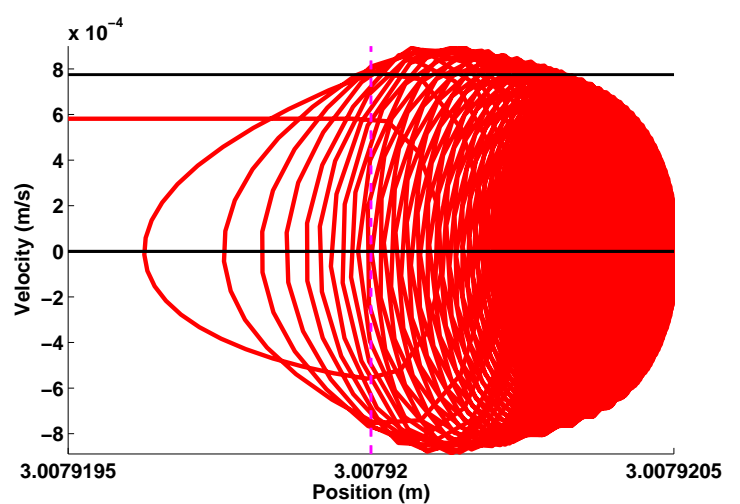

(a)

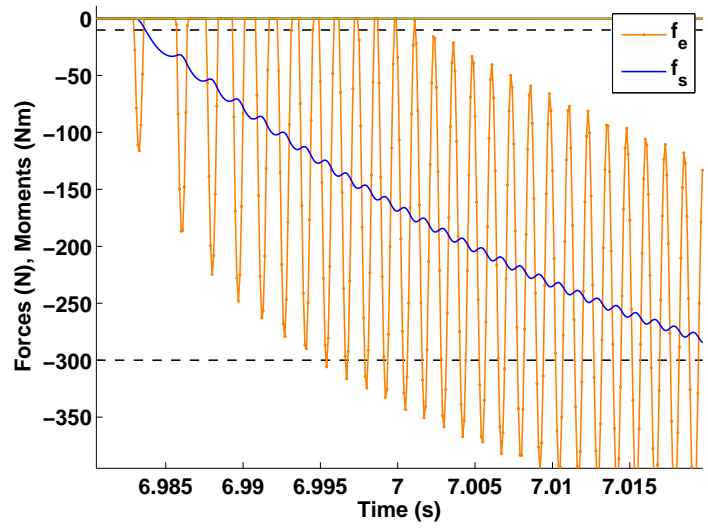

(b)

Figure 8.20: Above nominal environment stiffness $k_{e}=10^{9} \mathrm{~N} / \mathrm{m}$ : (a) Constrained trajectory $\left(x_{2}, \dot{x}_{2}\right)$ of the first mass. (b) System forces $f_{s}$ and $f_{e}$ close to the switching boundary.

expected, $k_{e}=10^{9} \mathrm{~N} / \mathrm{m}$, such that $k_{e} / k_{s} \approx 48$. In effect, this test is a more extreme version of the first base test depicted in Figure 8.5 when $k_{e}=5 \cdot 10^{8} \mathrm{~N} / \mathrm{m}$. As is the case for the environment stiffness tests of Chapter 6 , a bumpless transfer is achieved here because the impact velocity is less than $\bar{\mu}_{s w}$, but the large ratio $k_{e} / k_{s}$ leads to extreme oscillations in the environment force with peaks exceeding $500 \mathrm{~N}$.

In summary, under the proposed hybrid control $u\left(\xi, f_{s}\right)$ the SPDM successfully completes the interaction task while achieving a bumpless transfer between reach and force control. Using given parameters of the SPDM system and then executing Algorithm 5.1, a set of logic control specifications were defined which generated the polytopic state space $\mathcal{P}$. This operating region accommodates the environment whose stiffness and position were unknown, but were constrained to a specific range. After triangulating $\mathcal{P}$ a set of reach controllers were synthesized, and a hybrid controller was constructed. Then via simulation it has been shown that in free motion the reach controllers drive the end effector through $\mathcal{P}$ into $\mathcal{P}^{3}$, while complying with the logic control specifications; this behaviour occurs over a wide range of values of $m_{1} / m_{2}$. During the transition phase, and inspite of variations in the environment parameters, reach controller $u_{r c p}^{10}$ was able to drive the SPDM to a point where the hybrid controller switches to force control, and regulation of the sensed force is achieved. Note that for this design, the peak transient force during regulation is dependent on the environment position, as well as the 
reach controller in $\mathcal{P}^{3}$. While variation of the ratio $k_{e} / k_{s}$ did not prevent bumpless transfer and the ensuing regulation of the sensed force, tests in which $k_{e} / k_{s}$ was large exhibited nonideal behaviour. Specifically, the second mass may bounce excessively and oscillations in the environment force are prominent. Finally, the presence of structural damping, not considered in the original control design, alters the dynamics under force control, but was not found to interfere with achieving bumpless transfer. 


\section{Chapter 9}

\section{Conclusions}

This thesis addresses the manipulator interaction task during which the manipulator end effector must navigate through free space, make contact with an environment, and maintain contact at a specified force. Additionally, a number of requirements pertaining to the transient behaviour of the system were specified; specifically, that the manipulator should not exceed certain position and velocity limits, that the impact velocity should not exceed a threshold, that a given force controller $u_{f}$ should be enabled to perform force regulation, and that the transition to the force controller should occur only once. The control objective was to design a reach controller $u_{r c p}$ and a switching logic to switch between $u_{r c p}$ and $u_{f}$ that guarantee the aforementioned behaviour for the closed-loop system. In Chapter 5 the control problem was formulated and solved within the context and principles of the RCP, a control methodology that emphasises the control of transient behaviour. The solution method consisted of two parts. First, a solution for achieving bumpless transfer from reach control to force control was obtained through Lyanpunov analysis of the constrained motion phase. An integral outcome of this analysis was the derivation of the least upper bound switching velocity $\bar{\mu}_{s w}$, which was found to be a characteristic property of the given force controller and switched-system architecture. It was shown that to achieve bumpless transfer, then the switching velocity $\mu_{s w}$ should be chosen as $\mu_{s w} \in\left(0, \bar{\mu}_{s w}\right)$. This result, among others, led to the definition of a set of logic control specifications and an operational state space $\mathcal{P}$ that restricts the manipulator states to trajectories that will achieve bumpless transfer. In this way, $\mathcal{P}$ itself guarantees in part the aforementioned transient requirements. Additionally, an algorithm was provided for selecting the logic control specifications using problem data. 
For the second stage of the solution, the methods of RCP were applied to obtain a piecewise continuous affine feedback defined within $\mathcal{P}$. The advantage of the reach controllers is that for any initial condition of the manipulator within $\mathcal{P}$, the resulting trajectory is guaranteed to make contact with the environment and safely switch to force control. Note that a guide for designing each reach controller is given in Chapter 6 . The final product of the solution is a hybrid control $u\left(\xi, f_{s}\right)$ consisting of the reach controllers, force controller, and switching logic. Lastly, the hybrid control was designed to operate under conditions where the environment stiffness and location varied over known ranges.

In Chapter 1 we discussed a solution to the bumpless transfer problem presented in Teel et al which was the primary inspiration for the present work. That solution had two limitations: i) The solution only accommodates a fixed set of environment parameters; ii) The set of initial conditions guaranteeing bumpless transfer is generally small, and depends on the environment stiffness. The proposed design improves on these limitations as follows. With respect to i), we consider an environment modelled as a spring whose stiffness and position are not exactly known, but vary within a known range. It was then shown in Lemmas 5.2 and 5.3 that sufficient conditions for bumpless transfer are satisfied, in light of stiffness variations, so long as the switching velocity $\mu_{s w}$ is chosen as $\mu_{s w} \in\left(0, \bar{\mu}_{s w}\right)$. From there the simplex $\mathcal{P}^{3}$ was designed to guarantee that the impact velocity is no greater than $\mu_{s w}$, and that a switch to force control cannot occur outside of the simplex (this is done by choosing $\rho_{2}>\rho_{e \max }+\frac{\left|f_{s w}\right|}{\bar{k}_{\text {min }}}$ ). With respect to ii), first it was shown in Lemma 5.1 that a suitable reach controller exists such that trajectories in $\mathcal{P}^{3}$ are guaranteed to cross the switching boundary $\Gamma_{s w}$, regardless of the actual environment stiffness and position. Since the remaining reach controllers are designed to drive trajectories into $\mathcal{P}^{3}$ from all other regions of the state space $\mathcal{P}$, then in fact the set of initial conditions that guarantee bumpless transfer are all non-contact initial conditions in $\mathcal{P}$. Ultimately, these results represent vast improvements over Teel's work as the improvement to i) conveys a degree of robustness in the design to parameter variation, while the improvement to ii) expands the solution space to the entire (non-contact) operational state space of the manipulator.

Following the theoretical development of this thesis, a full interaction task was simulated for two manipulator systems where contact with an environment was made in the normal direction along the $x$-axis. The first system was a three-link manipulator with a 'second mass', whose 
definition was completely arbitrary, and the second was the SPDM, which is a real manipulator defined by specific physical parameters. Both were feedback linearised to grant direct access to the task space variables, in particular the $x$-coordinate of the end effector position and its velocity. The proposed methodology was applied along this dimension.

Simulations for both systems yielded similar results: assuming a set of nominal system parameters the reach controllers guided the end effector to the switching boundary, which prompted a transition to the force controller, which was followed by contact force regulation. No bouncing in the control occurred in the set of baseline tests. Additional tests introduced parameter variations in the manipulator and environment, or explored the effects of tuning the controllers. These tests all proved to be successful in achieving a bounceless transition in the control, but they also served to highlight additional benefits and limitations to the proposed design. For example, it was observed that the peak contact force depended in part on the position of the environment, which follows from the architecture of the proposed operating region. Moreover, the reach controller active during impact may be tuned to decrease the induced peak force at the cost of longer maneuver times. Surprisingly, the mass ratio $m_{1} / m_{2}$, where $m_{2}$ is the mass of the end effector tip and $m_{1}$ is a virtual mass parameter representing the bulk of the manipulator, did not play as significant a role as expected. Through simulation it was found that a ratio of $m_{1} / m_{2}=5$ was sufficient to justify certain modelling assumptions regarding control design.

Alternatively, one of the most important factors was the ratio $k_{e} / k_{s}$ of environment to sensor stiffness. Specifically, it was seen that when $k_{e} / k_{s}$ was larger than approximately 10, the smaller second mass would oscillate excessively upon initial impact, inducing undesirable physical bounces and peak environment forces (a bumpless transfer was still achieved however). This behaviour represents a limitation in the design best explained by two modelling features: i) the neglecting of the dynamics of the second mass, and ii) the inclusion of the sensor compliance in the motion of the masses. The justification for these two features are that they allow the sensed force to be defined in terms of the states of the first mass, $\xi$, and also allow for the adoption of a simplistic PD force controller $u_{f}$. Consequently, given the form of $u_{f}$, one could use the relationship between $\xi$ and the sensed force to derive conditions required to prevent a bounce in the control. These conditions are summarised in the lemmas presented in Chapter 
5. Moreover, these conditions in turn informed the selection of the logic control specifications, which were expressed with respect to $\xi$.

Inspite of the above limitation, the core contribution of this thesis is a unique approach towards the control design of interaction tasks that involves a synthesis of the free motion, transition, and constrained motion phases through the logic control specifications and reach controllers. Presumably, given the selection of a force controller better suited to a more detailed linear model, a similar process to the one here may be followed whereby a set of logic control specifications are selected to achieve, amongst other requirements, bumpless transfer.

This thesis provides a first glimpse into the application of the reach control problem to manipulator interaction tasks, and therefore opens the door to a number of future research avenues. As a first step, some of the models employed in this thesis may be enhanced for improved accuracy. For example, one can obtain a more accurate and detailed model for the sensed force, one that includes, say, a damping component, and then develop a methodology similar to that in Chapter 5. An enhancement in model detail may lead to more complex switching logic that contributes to achieving bumpless transfer. Then, the reach control problem can be used to reliably guide the manipulator to states wherein this switching logic is satisfied.

As another future topic, one may select a different force regulation scheme with which the techniques of the reach control problem may be integrated. One such example is the technique called accommodation control, or force/moment accommodation (FMA). When employed, the manipulator is typically rate-controlled in free motion. Upon contact the sensed force is converted into a velocity value, and an error is computed between the commanded end effector velocity and the converted velocity; when no contact force exists the commanded velocity is simply unmodified (see [16] and [17] for details). FMA is attractive for space-based tasks where it can be used to limit peak contact forces and ensure precise alignment during insertion operations. It is currently implemented on the SPDM and other manipulators on the International Space Station [18]-[21]. The integration of the force regulation principles of FMA with RCP's applicability to control of transient behaviour and complex tasks poses an interesting opportunity.

A third avenue involves the control of the manipulator itself. In this thesis task space control was utilized in which three of the six possible dimensions were controlled, only one 
of which employed RCP. The application of RCP in all six task space dimensions may allow for the execution of more complex maneuvers, and therefore give way to new and interesting robotic tasks that otherwise may not be feasible due to safety constraints and transient limits. Finally, in this thesis direct control of the torque at each joint was assumed, while in the case of the SPDM and manipulators in general each joint is actuated by a motor. Control at the 'motor level' is obviously well known as are individual path-planning techniques. However, consolidation of these control methods with those of RCP poses a challenge. In particular, one my choose to directly apply RCP at the actuator level to obtain some desired behaviour. Alternatively, RCP may be employed for a set of task space variables, producing a set of abstract reach controllers. The function of the reach controllers must then be realized through the synthesis of a control for the actuators. Finally, one could computationally generate a trajectory of the actuated joints that correspond to the evolution of a task space variable under reach control. Such a trajectory could then possibly serve as a reference for path-planning using an implementable controller. 


\section{Bibliography}

[1] Krishnaa Mehta. A Topological Obstruction in a Control Problem. M.Sc. thesis, University of Toronto, 2012.

[2] M. Helwa. Reach Control Problems on Polytopes. Ph.D. thesis, University of Toronto, 2013.

[3] Graeme Ashford. A Time-Varying Feedback Approach to Reach Control on a Simplex. M.Sc. thesis, University of Toronto, 2011.

[4] M. Ganness. Reach Control on Simplices by Piecewise Affine Feedback. M.Sc. thesis, University of Toronto, 2010.

[5] S. Greene. System Identification of SPDM Ground Test Facility Simulators. M.Sc. Thesis, University of Toronto, 2006.

[6] P. R. Pagilla and B. Yu A Hybrid A Stable Transition Controller for Constrained Robots. IEEE/ASME Transactions on Mechatronics. Vol. 6, No. 1, pp. 65-74, 2001.

[7] R. Carloni, R. Sanfelice, A. R. Teel and C. Melchiorri. A Hybrid Control Strategy for Robust Contact Detection and Force Regulation. Proceedings of the 2007 American Control Conference. New York, pp. 1461-1466, 2007.

[8] T. Tarn, Y. Wu, N. Xi and A. Isidori. Force Regulation and Contact Transition Control IEEE Control Systems Magazine. Vol. 16, pp 32-40, 1996.

[9] O. Khatib and J. Burdick. Motion and force control of robot manipulators. Proceedings of the IEEE International Conference of Robotics and Automation. pp 1381-1386, 1986. 
[10] R. Volpe and P. Khosla. A theoretical and experimental investigation of impact control for manipulators. Interational Journal of Robotics Research Vol. 12, no. 4, pp. 351-365, Aug. 1993.

[11] G.T. Marth, T.J. Tarn and A.K. Bejczy. Stable Phase Transition Control for Robot Arm Motion. Proceedings of the IEEE International Conference on Robotcis and Automation. Atlanta, Georgia, pp. 355-362, 1993.

[12] M.C.Çavuşoğlu, J. Yan and S. Shankar Sastry. A Hybrid System Approach to Contact Stability and Force Control in Robotic Manipulators. Proceedings of the 12th IEEE International Symposium on Intelligent Control. Istanbul, July 1997.

[13] G. Ferretti, G. Magnani and A. Zavala Rio. Impact Modelling and Control for Industrial Manipulators. IEEE Control Systems. Vol. 18, no. 4, pp. 65-71, Aug. 1998.

[14] J. K. Mills and D. M. Lokhorst. Stability and Control of Robotic Manipulators During Contact/Noncontact Task Transition. IEEE Transaction on Robotics and Automation Vol. 9, no. 3, pp. 335-345, 1993.

[15] N. Hogan. Impedance Control: An approach to Manipulation: Part I Theory. ASME Journal of Dynamic Systems, Measurement and Control, Vol. 107, pp 335-345, 1985.

[16] D.E. Whitney. Force Feedback control of manipulator fine motions. Journal of Dynamic Systems, Measurement, and Control. pp. 91-97, June 1977.

[17] M.M.R.L. Williams II, J.M. Henry, and D. Repperger. Free and constrained motion teleoperation via naturally-transitioning rate-to-force control. Proceedings of the IEEE International Conference on Robotics and Automation, pp. 225-230, May 1999.

[18] R. Mukherji, D. Rey, M. Stieber and J. Lymer. Special Purpose Dexterous Manipulator (SPDM) - Advanced Concepts, Features and Development Test Results. Proceedings of the 6th International Symposium on Artificial Intelligence, Robotics and Automation in Space, iSAIRAS. St. Hubert, 2001.

[19] R. Mukherji and J. Lymer. Results of Human-In-The-Loop Testing of the Force/Moment Accommodation Feature Using the Special Purpose Dexterous Manipulator Ground Test 
Bed. Proceedings of the SAE International Conference on Environmental Systems, SAE. Toulouse, France, 2000.

[20] F. Aghili, E. Dupuis, E. Martin, and J-C. Piedboeuf. Force/Moment Accommodation Control for Tele-Operated Manipulators Performing Contact Tasks in Stiff Environment. Proceedings of the 2001 IEEE/RSJ International Conference on Intelligent Robots and Systems, Maui, Hawaii, 2001.

[21] G. Jorgensen and E. Bains. SRMS History, Evolution and Lessons Learned. AIAA Space 2011 Conference and Exposition. Long Beach, California, 2011.

[22] J. Hudson. Piecewise Linear Topology. University of Chicago Lecture Notes. W.A. Benjamin Inc. 1969.

[23] C. W. Lee. Subdivisions and triangulations of polytopes. Handbook of Discrete and Computational Geometry. CRC Press Series Discrete Math. Appl., pp. 271-290, 1997.

[24] A. Berman and R.J. Plemmons. Nonnegative Matrices in the Mathematical Sciences. Academic Press, New York. 1979.

[25] R.T Rockafellar. Convex Analysis. Princeton University, Princeton, New Jersey 1970.

[26] A. Brondsted. An Introduction to Convex Polytopes. Springer-Verlag, New York Inc., 1983.

[27] V.M. Buchstaber and T.E. Panov. Torus Actions and their Applications in Topology and Combinatorics. University Lecture Series, vol. 24. American Mathematical Society, 2002.

[28] L.C.G.J.M. Habets and J.H. van Schuppen. Control of piecewise-linear hybrid systems on simplices and rectangles, in: M.D. Di Benedetto and A.L. Sangiovanni- Vincentelli (Eds.) Hybrid Systems: Computation and Control, Lecture Notes in Computer Science. Springer Verlag, vol. 2034, pp. 261-274, 2001.

[29] L.C.G.J.M. Habets and J.H. van Schuppen. A control problem for affine dynamical systems on a full-dimensional polytope. Automatica. no. 40, pp. 21-35, 2004. 
[30] L.C.G.J.M. Habets, P.J. Collins, and J.H. van Schuppen. Reachability and control synthesis for piecewise-affine hybrid systems on simplices. IEEE Transactions Automatic Control. no. 51 , pp. $938-948,2006$.

[31] B. Roszak and M. E. Broucke. Necessary and sufficient conditions for reachability on a simplex. Automatica. vol. 42, no. 11, pp. 1913-1918, November 2006.

[32] M.E. Broucke and M. Ganness. Reach control on simplices by piecewise affine feedback. IEEE Transactions on Automatic Control. In revision, April 2012.

[33] M.E. Broucke. Reach control on simplices by continuous state feedback. SIAM Journal on Control and Optimization. vol. 48, issue 5, pp. 3482-3500, February 2010.

[34] O. Toker and H. Ozbay. On the NP-hardness of solving bilinear matrix inequalities and simultaneous stabilization with static output feedback. Proceedings of the 1995 American Control Conference, Seattle, Washington, 1995.

[35] M. Spong, S. Hutchinson, and M. Vidyasagar. Robot Modeling and Control. Wiley, 2006.

[36] R. M. Murray, Z. X. Li, and S. S. Sastry. A Mathematical Introduction to Robotic Manipulation. CRC Press, 1994.

[37] R. Mukheri. Internal Report. MDA Corporation, Brampton Ontario. 2011.

[38] SPDM Simulator. MDA Corporation, Brampton Ontario. 2013.

[39] P.C. Hughes, G.B. Sincarsin, and G.M.T. D'Eleuterio. Dynamics of Elastic Chains. Dynacon Report SS-102, Dynacon Enterprises Limited, 1988.

[40] T. Melzer. SVD and its Application to Generalized Eigenvalue Problems. http:// www.ipf.tuwien.ac.at/ tm/svd.pdf, June 2004.

[41] S. D. Eppinger and W. P. Seering. On Dynamic Models of Robot Force Control. Proceedings of the 1986 IEEE International Conference on Robotics and Automation, San Francisco, California, 1986. 